Lawrence Berkeley National Laboratory Report

LBNL - 51432

\title{
Particle Deposition from Turbulent Flow: Review of Published Research and Its Applicability to Ventilation Ducts in Commercial Buildings
}

\author{
Mark R. Sippola ${ }^{1}$ and William W. Nazaroff ${ }^{1,2}$ \\ ${ }^{1}$ Environmental Engineering Program \\ Department of Civil and Environmental Engineering \\ University of California \\ Berkeley, CA 94720-1710 \\ ${ }^{2}$ Indoor Environment Department \\ Environmental Energy Technologies Division \\ Lawrence Berkeley National Laboratory \\ Berkeley, CA, 94720-1710
}

June 2002

Draft 


\section{Abstract}

This report reviews published experimental and theoretical investigations of particle deposition from turbulent flows and considers the applicability of this body of work to the specific case of particle deposition from flows in the ducts of heating, ventilating and air conditioning (HVAC) systems. Particle deposition can detrimentally affect the performance of HVAC systems and it influences the exposure of building occupants to a variety of air pollutants.

The first section of this report describes the types of HVAC systems under consideration and discusses the components, materials and operating parameters commonly found in these systems. The second section reviews published experimental investigations of particle deposition rates from turbulent flows and considers the ramifications of the experimental evidence with respect to HVAC ducts. The third section considers the structure of turbulent airflows in ventilation ducts with a particular emphasis on turbulence investigations that have been used as a basis for particle deposition models. The final section reviews published literature on predicting particle deposition rates from turbulent flows.

A large quantity of experimental data regarding particle deposition from turbulent flows has been collected using a range of techniques of varying quality. Nearly all of these data have been collected from straight tubes or ducts with a fully developed turbulent flow profile and the data are widely scattered. Most of the data of acceptable quality have been collected from tubes or ducts with hydraulic diameters much smaller than ducts in typical HVAC systems. Particle deposition from turbulent flow with a developing flow profile has not been systematically investigated and only two investigations of particle deposition from turbulent flow through bends have been published. Developing turbulent flow profiles and bends are common in HVAC ducts.

Owing to the large number of investigations into particle deposition from turbulent flow, much is known; however, the direct applicability to the case of particles in HVAC ducts is limited. Particle size, turbulence intensity and the roughness and orientation (horizontal or vertical) of the deposition surface are the parameters that control particle deposition rates and all of these factors are likely to be pertinent in HVAC ducts. Particle diameters of concern in HVAC ducts range from about 0.003 to $30 \mu \mathrm{m}$ and deposition rates are known to vary strongly in this range. Friction velocities in HVAC ducts are 
likely to be in the range $0.1-1 \mathrm{~m} / \mathrm{s}$ and variations of turbulence intensities in this range are likely to influence deposition rates. Both microscale surface roughness (from less than 1 micron up to hundreds of microns) and macroscale roughness (about $1 \mathrm{~mm}$ and larger) have been demonstrated to enhance particle deposition relative to the case of a smooth surface. Microscale roughness intrinsic to the duct material, or due to corrosion or previous deposition of particles, and macroscale roughness from thermal insulation, joints between duct sections and debris are all potentially important in HVAC ducts. The floors of horizontal ducts are likely to experience higher rates of particle deposition than vertical duct walls or horizontal duct ceilings owing to the influence of gravity on large particles. With respect to deposition in HVAC ducts, the overall experimental data set is most lacking in information regarding the influence of developing turbulent flow profiles, microscale roughness and duct bends on particle deposition.

Particle deposition from turbulent flow depends on the nature of the turbulent flow field. Interactions between particles and air turbulence frequently determine particle deposition rates. Properties of turbulent flow that have been incorporated into particle deposition models include the fluctuating velocity component normal to the wall and the eddy viscosity. Coherent structures in near-wall turbulence such as low-speed axial streaks, near-wall streamwise vortices, bursts and downsweeps have been theorized to be important for the deposition of certain sized particles. A representative fraction of experiments and numerical simulations investigating these turbulent properties and structures are described. Turbulence near both smooth and rough walls is addressed; however, investigations into turbulence near smooth walls are more substantial and consistent in their findings. These descriptions of turbulent flow provide a foundation for understanding the theory behind particle deposition models and the results of numerical simulations of particle deposition from turbulent flows.

Four broad methods of predicting particle deposition rates are found in the literature: empirical equations, Eulerian models, sublayer models and Lagrangian simulations. These methods usually require information about the particle size and density, as well as the air speed and dimensions of the duct containing the flow. Deposition rates are most commonly reported in the form of the dimensionless deposition velocity, $V_{d}{ }^{+}$, versus the dimensionless relaxation time, $\tau^{+}$, a measure of particle inertia.

Empirical equations are simple best fits to experimental data and are easy to apply. They can be combined and applied to a broad range of particle sizes and they can also be 
applied to both vertical and horizontal deposition surfaces. They are of limited value for rough deposition surfaces because of sparse experimental data. Empirical equations are the only type of predictive method that has been developed for particle deposition from turbulent flow in bends. While offering little fundamental understanding, certain empirical equations can be applied to aspects of particle deposition in HVAC ducts with reasonable confidence.

Eulerian models include gradient diffusion models, free-flight models and turbophoretic models, all of which are quickly solvable with current computing power. Gradient diffusion models, when applied with reasonable assumptions, are unlikely to perform well over the full range of particle sizes for deposition in HVAC ducts. Some free-flight models successfully predict particle deposition rates through a combination of theory and empiricism. Free-flight models as presented in the literature are solely applicable to vertical deposition surfaces, though they could be adapted to horizontal surfaces. Some free-flight models achieve moderate success predicting deposition to rough surfaces by a simple modification to the method of predicting deposition to smooth surfaces. Recommended free-flight models may yield reasonable predictions in HVAC ducts, but there is little advantage to these models over the recommended empirical equations. Turbophoretic models are a significant improvement over gradient diffusion and freeflight models and are the models that are most appropriate for application to HVAC ducts. These models are applicable to vertical and horizontal surfaces over the entire range of particle sizes. Turbophoretic models are able to account for a broader range of particle transport mechanisms than other Eulerian models. They achieve good agreement with the trends and magnitudes of the experimental data with only a small amount of empiricism. The same simple method of accounting for surface roughness is used in turbophoretic models as in free-flight models.

Sublayer models are semi-Lagrangian models that can be solved rapidly with current computing power to give reasonable predictions of particle deposition to vertical and horizontal surfaces. To account for surface roughness, some sublayer models apply a similar method as the simple method used in free-flight models. Some of these models achieve reasonable agreement with the magnitudes of experimentally observed deposition rates and recommended sublayer models may be applied to the case of deposition in HVAC ducts with reasonable expectations about their performance. 
Lagrangian simulations of particle deposition have included those conducted in relatively simple modeled turbulent flows to highly detailed flows generated by large eddy simulation (LES) and direct numerical simulation (DNS). Lagrangian simulations are often considered 'numerical experiments' because the results are for discrete particle sizes and specific to the numerically simulated flow conditions, much like physical experiments. The results of Lagrangian simulations are valuable for informing expectations about particle deposition in HVAC ducts; however, the high level of computational power required by these simulations makes them unsuitable at present for predicting particle deposition rates under the variety of conditions found in buildings. These simulations provide vast amounts of information on the forces acting on particles, particle velocities and particle deposition rates and offer insight into the factors that contribute to experimental uncertainty. The results of Lagrangian simulations of particle deposition generally agree with the trends and magnitude observed in experiments for both vertical and horizontal surfaces. The single reported Lagrangian simulation to consider roughness at the deposition surface resulted in the same trends as observed in experiments and also in Eulerian models using similar methods.

Turbophoretic models are the best models for application to the case of particle deposition in HVAC ducts. Turbophoretic models offer accuracy similar to other models when predicting particle deposition rates; however, they offer greater versatility in application and are based on physically realistic assumptions. Turbophoretic models have two main limitations when being applied to HVAC ducts. First, the models assume a fully developed turbulent flow profile which is not appropriate throughout HVAC systems. Second, the method of accounting for surface roughness is somewhat simplistic and corroborated by only a single data set. While models do capture the broad trends seen in experiments, they can deviate markedly from observations. In general, existing modeling approaches and empirical data are not sufficient to reliably predict particle deposition in HVAC ducts. Lastly, obtaining accurate input information for predicting particle deposition rates is a concern regardless of the type of predictive method used. 


\section{Table of Contents}

$\begin{array}{ll}\text { ABSTRACT } & \text { iii }\end{array}$

TABLE OF CONTENTS vii

LIST OF FIGURES $\quad$ xi

LIST OF TABLES $\quad$ xvi

NOMENCLATURE Xvii

ACKNOWLEDGEMENTS Xxii

1. INTRODUCTION TO PARTICLES AND HVAC SYSTEMS 1

1.1. Particles and Human Health 1

1.2. Anti-Terrorism Concerns: Chemical and Biological Agents 2

1.3. HVAC Systems and Indoor Air Quality 3

1.4. Types of HVAC Systems 4

1.5. HVAC System Components and Particle Deposition 5

1.5.1. Outside air louvers, filters, cooling and heating 6

$\begin{array}{ll}\text { 1.5.2. Supply fan and ventilation ducts } & 7\end{array}$

$\begin{array}{ll}\text { 1.5.3. Duct components and terminal devices } & 8\end{array}$

1.5.4. Summary 9

2. EXPERIMENTAL WORK ON PARTICLE DEPOSITION FROM TURBULENT TUBE AND DUCT FLOWS 10

2.1. Definition of Parameters 10

2.2. Experimental Data from Straight Tubes and Ducts 15

2.3. Details about Experiments in Straight Tubes and Ducts 17

2.4. Historical Development of Experiments in Straight Tubes and Ducts 20 
$\begin{array}{ll}\text { 2.4.2. Microscale roughness } & 21\end{array}$

2.4.3. Fibrous and macroscale roughness 21

2.5. Particle Deposition in Tube Bends 22

2.6. Relevance of Current Data to Deposition in Ventilation Ducts 24

3. TURBULENT AIRFLOW IN VENTILATION DUCTS 28

3.1. Description of Turbulent Flow near Smooth Walls 27

3.1.1. Fluctuating velocity component normal to a smooth wall 28

3.1.2. Eddy viscosity 31

3.1.3. Organized structures in turbulence near a smooth wall 33

3.2. Description of Turbulent Flow near Rough Walls 35

3.2.1. Turbulence in rough wall boundary layers: Experiments 35

3.2.2. Turbulence in rough walled channels and pipes: Experiments 37

3.2.3. Turbulence in rough walled channels and pipes: Simulations 37

3.3. Secondary Flows 38

3.4. Turbulent Airflow Summary 39

4. PREDICTING PARTICLE DEPOSITION RATES 40

4.1. Particle Transport Mechanisms $\quad 40$

4.1.1. Brownian diffusion 41

4.1.2. Drag force 41

4.1.3. Gravitational force 42

4.1.4. Shear-induced lift force 43

4.1.5. Thermophoresis 44 
4.1.7.Turbulent diffusion $\quad 46$

4.1.8. Turbophoresis 47

4.1.9. Combining transport mechanisms 48

4.1.10. Other transport mechanisms 48

4.2. Methods: Empiricism, Eulerian Models and Lagrangian Simulation 48

4.3. Empirical Equations 49

4.3.1. Empirical equations for smooth surfaces in the diffusion regime $\quad 50$

4.3.2. Empirical equations for smooth surfaces in the diffusionimpaction regime 53

4.3.3. Empirical equations for smooth surfaces in the inertia moderated regime $\quad 54$

4.3.4. Synthesis of empirical equations 56

4.3.5. Empirical equations for rough surfaces 57

4.4. Eulerian Models 58

4.4.1. Free-flight models for smooth surfaces $\quad 59$

4.4.2. Free-flight models for rough surfaces 64

4.4.3. Gradient diffusion models 68

4.4.4. Turbophoretic models 71

$\begin{array}{ll}\text { 4.5. Sublayer Models } & 75\end{array}$

$\begin{array}{ll}\text { 4.6. Lagrangian Simulations } & 79\end{array}$

4.6.1. Lagrangian simulations with stochastically modeled turbulent flow 80

4.6.2. Lagrangian simulations with LES and DNS 85

4.7. Empirical Equations for Particle Deposition in Duct Bends 92 


\section{List of Figures}

Figure 1. Schematic diagram of airflow through the mechanical room for a typical central HVAC system.

Figure 2. Experimental particle deposition data collected from turbulent flow through small vertical tubes by several researchers.

Figure 3. Experimental particle deposition data collected from flow through vertical smooth and rough tubes in the diffusion-impaction and inertia-moderated regimes.

Figure 4. Experimental particle deposition data collected by Wells \& Chamberlain (1967) for particles depositing to vertically oriented smooth brass and fibrous filter paper with roughness length scale, $k$, of approximately $100 \mu \mathrm{m}$.

Figure 5. Experimental particle deposition data for particles depositing to a smooth duct floor and a duct floor covered with artificial grass at different values of the friction velocity, $u^{*}$.

Figure 6. Experimental data collected for particle penetration through 90-degree bends with turbulent flow and small tube diameters.

Figure 7. Experimental data collected for deposition in 90-degree bends with turbulent flow and small tube diameters compared to experimental data collected in straight tubes.

Figure 8. Experimental particle deposition data for particles depositing to the smooth floors of large ducts.

Figure 9. Experimental particle deposition data collected by Sehmel (1973) for particles depositing to the floor and ceiling of a horizontal duct at three different values of the friction velocity, $u^{*}$.

Figure 10. Particle deposition data for polydisperse aerosols in straight $30 \mathrm{~cm}$ square ducts measured by inferring deposition from differences in concentration measurements. 
Figure 11. Definition of coordinate directions in turbulent duct flow.

Figure 12. Profiles of $v_{r m s}^{+}$versus $y^{+}$derived from measurements in pipe flow and DNS of channel flow. The bottom panel shows the same data as the top panel with the axes scaled to focus attention near the wall.

Figure 13. Comparison of correlations for eddy diffusivity versus dimensionless distance from a wall.

Figure 14. Approximate instantaneous arrangement and magnitudes of alternating low-speed and high-speed streaks of fluid near a wall in turbulent flow.

Figure 15. Schematic of near-wall turbulence illustrating the association between low-speed streaks, streamwise vortices, bursts and down sweeps.

Figure 16. Secondary flow in the $y-z$ plane of a straight rectangular duct with fully developed turbulent flow.

Figure 17. Secondary flow established in the $y-z$ plane in a leftward turning bend with the outside of the bend to the right.

Figure 18. Concentration profile of a diffusive species based on the assumptions of the film model.

Figure 19. Comparison of empirical expressions for particle dimensionless deposition velocities in the diffusion regime.

Figure 20. Comparison of empirical expressions for particle dimensionless deposition velocities in the diffusion-impaction regime.

Figure 21. Comparison of empirical expressions for particle dimensionless deposition velocities in the inertia-moderated regime.

Figure 22. Comparison of empirical expressions for particle dimensionless deposition velocities to horizontal floor and ceiling surfaces.

Figure 23. Comparison of free flight models in the case of deposition to a smooth vertical surface. 
Figure 24. Description of parameters to determine the particle capture distance to a rough surface by the method recommended by Browne (1974).

Figure 25. Comparison of free-flight models of Browne (1974) and ElShobokshy \& Ismail (1980) with experimental data in the case of deposition to a rough vertical surface.

Figure 26. Comparison of free-flight models of Wood (1981a) and Im \& Ahluwalia (1989) with experimental data in the case of deposition to a rough vertical surface.

Figure 27. Comparison of gradient diffusion models in the case of a smooth vertical surface.

Figure 28. Comparison of the turbophoretic model of Guha (1997) in the case of a smooth vertical surface including and excluding the effect of turbophoresis.

Figure 29. Application of the turbophoretic model of Guha (1997) with different roughness values to the conditions of the experiments of Liu \& Agarwal (1974).

Figure 30. Schematic of near-wall turbulence modeling by two-dimensional stagnation point flow in sublayer models. A particle depositing at the limiting trajectory is also shown.

Figure 31. Application of the sublayer model of Fan \& Ahmadi (1993) with different roughness values to the conditions of the experiments of Liu \& Agarwal (1974).

Figure 32. Results for deposition velocity to a smooth vertical surface by the Lagrangian simulation of Kallio \& Reeks (1989) with and without the lift force.

Figure 33. Results for deposition velocity to a smooth vertical surface by the Lagrangian simulation of Li \& Ahmadi (1993a) for particle densities of 900 and $2400 \mathrm{~kg} / \mathrm{m}^{3}$. 
Figure 34. Results for deposition velocity to a smooth floor surface by the Lagrangian simulation of Li \& Ahmadi (1993a) compared to the similar experiments of Sehmel (1973).

Figure 35. Results for deposition velocity to a smooth vertical surface by the Lagrangian simulation of Li \& Ahmadi (1993a) allowing for particle bounce with different restitution coefficients, $r$.

Figure 36. Results for deposition velocity to smooth and rough vertical surfaces by the Lagrangian simulation of Li \& Ahmadi (1993b) with four different roughness values.

Figure 37. Results for deposition velocity to rough horizontal floor surfaces by the Lagrangian simulation of Li \& Ahmadi (1993b) with four different roughness values.

Figure 38. Results for deposition to a smooth vertical surface in the simulation of Chen \& Ahmadi (1997) showing the influence of gravity in a vertical flow through the shear-induced lift force.

Figure 39. Results of the DNS-Lagrangian simulation of McLaughlin (1989) with and without the lift force compared to the experiments of Liu \& Agarwal (1974).

Figure 40. Results of the DNS-Lagrangian simulation of Ounis et al. (1993) for particles in the diffusive regime compared to the empirical equation (52) with $k_{l}=0.084$.

Figure 41. Results of the DNS-Lagrangian simulation of Chen \& McLaughlin (1995) with wall-corrected drag and optimum lift compared to the experiments of Liu \& Agarwal (1974).

Figure 42. Results of the LES-Lagrangian simulation of Wang \& Squires (1996b) with and without the lift force compared to the experiments of Liu \& Agarwal (1974).

Figure 43. Results of the DNS- and LES-Lagrangian simulations of Uijttewaal \& Oliemans (1996) with drag force only compared to the experiments of Liu \& Agarwal (1974). 
Figure 44. Results of the LES-Lagrangian simulation of Wang et al. (1997) with and without the optimum lift force compared to the experiments of Liu \& Agarwal (1974).

Figure 45. Results of the DNS-Lagrangian simulation of Zhang \& Ahmadi (2000) with downward and horizontal flow for deposition to a vertical wall surface at low friction velocities compared to the experiments of Liu \& Agarwal (1974).

Figure 46. Results of the DNS-Lagrangian simulation of Zhang \& Ahmadi (2000) for deposition to a vertical wall from upward flow and downward flow at high friction velocities compared to the experiments of Liu \& Agarwal (1974).

Figure 47. Results of the DNS-Lagrangian simulation of Zhang \& Ahmadi (2000) for deposition to a horizontal floor at two friction velocities compared to the analogous experiments of Sehmel (1973).

Figure 48. Summary of results for deposition to smooth vertical surfaces from several Lagrangian simulations.

Figure 49. Comparison of empirical model predictions for particle penetration through a $90^{\circ}$ bend in a $15-\mathrm{cm}$ square duct at a velocity of $5 \mathrm{~m} / \mathrm{s}$. 


\section{List of Tables}

Table 1. Measured densities of dust on supply duct floors in office buildings and schools.

Table 2. Equations for the Fanning friction factor in ducts with rough walls.

Table 3. Particle deposition experiments in horizontal tubes with hydraulic diameter less than $2.7 \mathrm{~cm}$.

Table 4. Particle deposition experiments in vertical tubes with hydraulic diameter less than $2.7 \mathrm{~cm}$.

Table 5. Particle deposition experiments in horizontal tubes with hydraulic diameter greater than $2.7 \mathrm{~cm}$.

Table 6. Particle deposition experiments in vertical tubes with hydraulic diameter greater than $2.7 \mathrm{~cm}$.

Table 7. Explanation of comments in Tables 3-6 and Table 8.

Table 8. Particle deposition experiments in tube bends with turbulent flow.

Table 9. Correlations for the eddy viscosity of air.

Table 10. Recommended values of $k_{l}$ for equation (50).

Table 11. Recommended values of $k_{2}$ for equation (51).

Table 12. Recommended values of $k_{3}$ for equation (52).

Table 13. Summary of assumptions for free-flight model by different investigators.

Table 14. Summary of conditions in Lagrangian particle deposition simulations with stochastically modeled turbulent flow.

Table 15. Summary of conditions in Lagrangian particle deposition simulations with turbulent flow from LES and DNS. 


\section{Nomenclature}

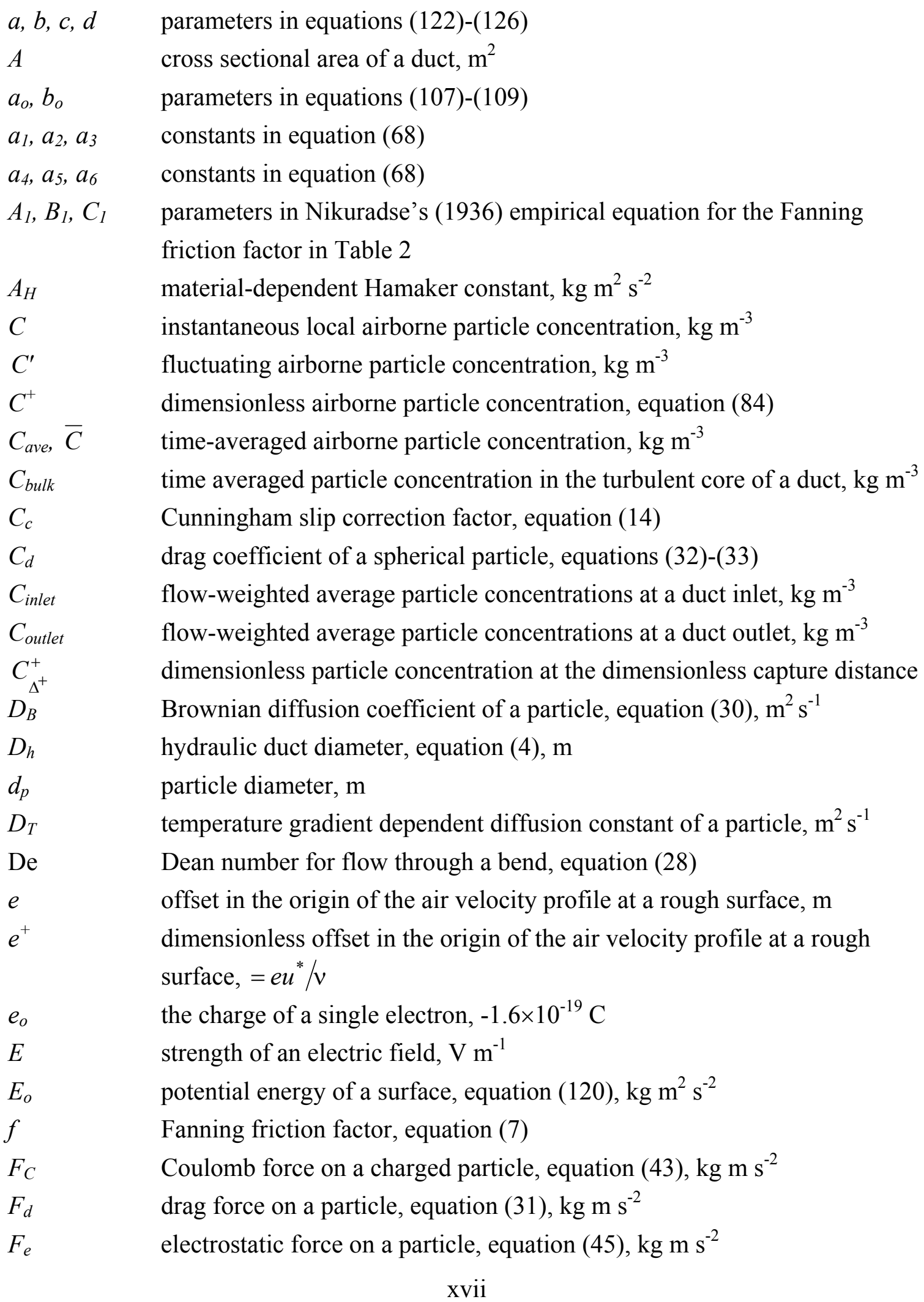




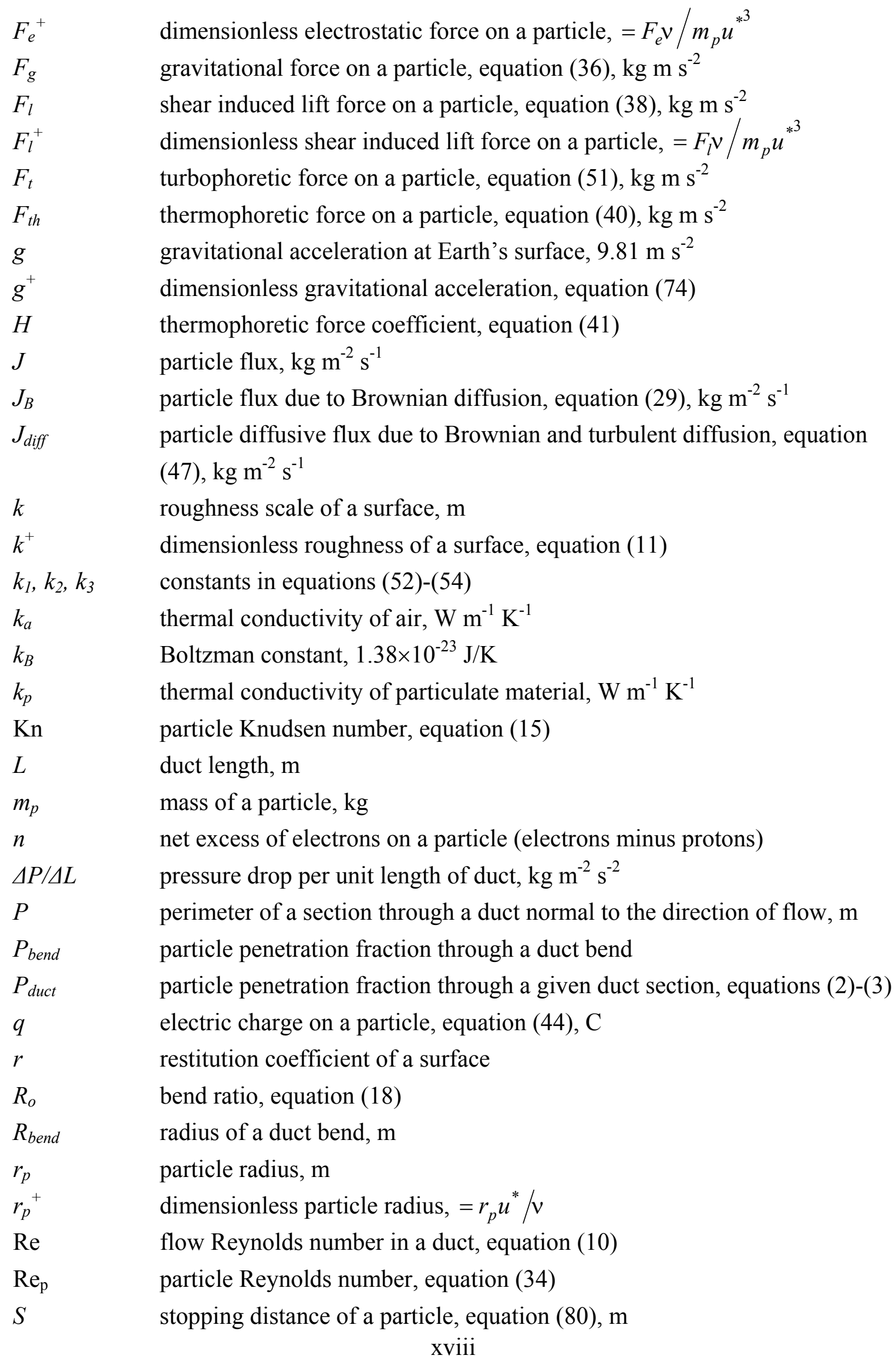

dimensionless electrostatic force on a particle, $=F_{e} v / m_{p} u^{*^{3}}$

$F_{g}$

$F_{l}$

$F_{l}^{+}$

$F_{t}$

$F_{\text {th }}$

$g$

$g^{+}$

$H$

$J$

$J_{B}$

$J_{\text {diff }}$

$k$

$k^{+}$

$k_{1}, k_{2}, k_{3}$

$k_{a}$

$k_{B}$

$k_{p}$

$\mathrm{Kn}$

$L$

$m_{p}$

$n$

$\Delta P / \Delta L$

$P$

$P_{\text {bend }}$

$P_{\text {duct }}$

$q$

$r$

$R_{o}$

$R_{\text {bend }}$

$r_{p}$

$r_{p}^{+}$

$\operatorname{Re}$

$\mathrm{Re}_{\mathrm{p}}$

$S$

gravitational force on a particle, equation (36), $\mathrm{kg} \mathrm{m} \mathrm{s}^{-2}$

shear induced lift force on a particle, equation (38), $\mathrm{kg} \mathrm{m} \mathrm{s}^{-2}$

dimensionless shear induced lift force on a particle, $=F_{l} v / m_{p} u^{* 3}$

turbophoretic force on a particle, equation (51), $\mathrm{kg} \mathrm{m} \mathrm{s}^{-2}$

thermophoretic force on a particle, equation (40), $\mathrm{kg} \mathrm{m} \mathrm{s}^{-2}$

gravitational acceleration at Earth's surface, $9.81 \mathrm{~m} \mathrm{~s}^{-2}$

dimensionless gravitational acceleration, equation (74)

thermophoretic force coefficient, equation (41)

particle flux, $\mathrm{kg} \mathrm{m}^{-2} \mathrm{~s}^{-1}$

particle flux due to Brownian diffusion, equation (29), $\mathrm{kg} \mathrm{m}^{-2} \mathrm{~s}^{-1}$

particle diffusive flux due to Brownian and turbulent diffusion, equation

(47), $\mathrm{kg} \mathrm{m}^{-2} \mathrm{~s}^{-1}$

roughness scale of a surface, $\mathrm{m}$

dimensionless roughness of a surface, equation (11)

constants in equations (52)-(54)

thermal conductivity of air, $\mathrm{W} \mathrm{m}^{-1} \mathrm{~K}^{-1}$

Boltzman constant, $1.38 \times 10^{-23} \mathrm{~J} / \mathrm{K}$

thermal conductivity of particulate material, $\mathrm{W} \mathrm{m}^{-1} \mathrm{~K}^{-1}$

particle Knudsen number, equation (15)

duct length, $m$

mass of a particle, $\mathrm{kg}$

net excess of electrons on a particle (electrons minus protons)

pressure drop per unit length of duct, $\mathrm{kg} \mathrm{m}^{-2} \mathrm{~s}^{-2}$

perimeter of a section through a duct normal to the direction of flow, $\mathrm{m}$

particle penetration fraction through a duct bend

particle penetration fraction through a given duct section, equations (2)-(3)

electric charge on a particle, equation (44), C

restitution coefficient of a surface

bend ratio, equation (18)

radius of a duct bend, $m$

particle radius, $\mathrm{m}$

dimensionless particle radius, $=r_{p} u^{*} / v$

flow Reynolds number in a duct, equation (10)

particle Reynolds number, equation (34)

stopping distance of a particle, equation (80), m

$\mathrm{xviii}$ 


\begin{tabular}{|c|c|}
\hline$S^{+}$ & dimensionless stopping distance of a particle, equation (81) \\
\hline Sc & particle Schmidt number, equation (55) \\
\hline St & particle Stokes number, equation (19) \\
\hline$T$ & absolute temperature, $\mathrm{K}$ \\
\hline$u$ & instantaneous streamwise air velocity, $\mathrm{m} \mathrm{s}^{-1}$ \\
\hline $\bar{u}$ & time averaged mean streamwise air velocity, $\mathrm{m} \mathrm{s}^{-1}$ \\
\hline$u^{\prime}$ & fluctuating streamwise air velocity, $\mathrm{m} \mathrm{s}^{-1}$ \\
\hline$u^{*}$ & friction velocity, equations (5)-(6), $\mathrm{m} \mathrm{s}^{-1}$ \\
\hline$\overline{u^{\prime} v^{\prime}}$ & $\begin{array}{l}\text { the time average of the product of the streamwise and wall normal } \\
\text { fluctuating air velocity components, } \mathrm{m}^{2} \mathrm{~s}^{-2}\end{array}$ \\
\hline$u_{a}$ & local air velocity, $\mathrm{m} \mathrm{s}^{-1}$ \\
\hline$U_{\text {ave }}$ & bulk average air velocity in a duct, $\mathrm{m} \mathrm{s}^{-1}$ \\
\hline$v$ & instantaneous wall normal air velocity, $\mathrm{m} \mathrm{s}^{-1}$ \\
\hline $\bar{v}$ & time averaged mean wall normal air velocity, $\mathrm{m} \mathrm{s}^{-1}$ \\
\hline$v^{\prime}$ & fluctuating wall normal air velocity, $\mathrm{m} \mathrm{s}^{-1}$ \\
\hline$\overline{v^{\prime} C^{\prime}}$ & $\begin{array}{l}\text { the time average of the product of the wall normal fluctuating air velocity } \\
\text { and the fluctuating airborne particle concentration, } \mathrm{kg} \mathrm{m}^{-2} \mathrm{~s}^{-1}\end{array}$ \\
\hline$V_{d}$ & particle deposition velocity, equation (1), $\mathrm{m} \mathrm{s}^{-1}$ \\
\hline$V_{d}^{+}$ & dimensionless particle deposition velocity, equation (12) \\
\hline$V_{d, d i f f}^{+}$ & dimensionless particle deposition velocity due to particle diffusion \\
\hline$V_{d, \text { inertial }}^{+}$ & dimensionless particle deposition velocity due to particle inertia \\
\hline$v_{g}$ & gravitational settling velocity of a particle, equation (37), $\mathrm{m} \mathrm{s}^{-1}$ \\
\hline$v_{p}$ & particle velocity, $\mathrm{m} \mathrm{s}^{-1}$ \\
\hline$v_{p, o}$ & initial particle velocity, $\mathrm{m} \mathrm{s}^{-1}$ \\
\hline$v_{p, \text { crit }}$ & $\begin{array}{l}\text { critical particle impact velocity for determining the likelihood of particle } \\
\text { bounce upon impact with a wall, equation (119), } \mathrm{m} \mathrm{s}^{-1}\end{array}$ \\
\hline$v_{p c y}$ & particle convective velocity in the wall-normal direction, $\mathrm{m} \mathrm{s}^{-1}$ \\
\hline$v_{p c y}^{+}$ & $\begin{array}{l}\text { dimensionless particle convective velocity in the wall-normal direction, } \\
=v_{p c y} / u^{*}\end{array}$ \\
\hline$v_{p x}$ & particle velocity in the axial direction, $\mathrm{m} \mathrm{s}^{-1}$ \\
\hline$v_{p x}^{+}$ & dimensionless particle velocity in the axial direction, $=v_{p x} / u^{*}$ \\
\hline$v_{p y}$ & particle velocity normal to the wall, $\mathrm{m} \mathrm{s}^{-1}$ \\
\hline$v_{p y}^{+}$ & dimensionless particle velocity normal to the wall, $=v_{p y} / u^{*}$ \\
\hline$v_{p y}^{\prime}$ & fluctuating particle velocity normal to the wall, $\mathrm{m} \mathrm{s}^{-1}$ \\
\hline$v_{p y, o}$ & initial particle velocity towards the wall in a sublayer model, $\mathrm{m} \mathrm{s}^{-1}$ \\
\hline
\end{tabular}




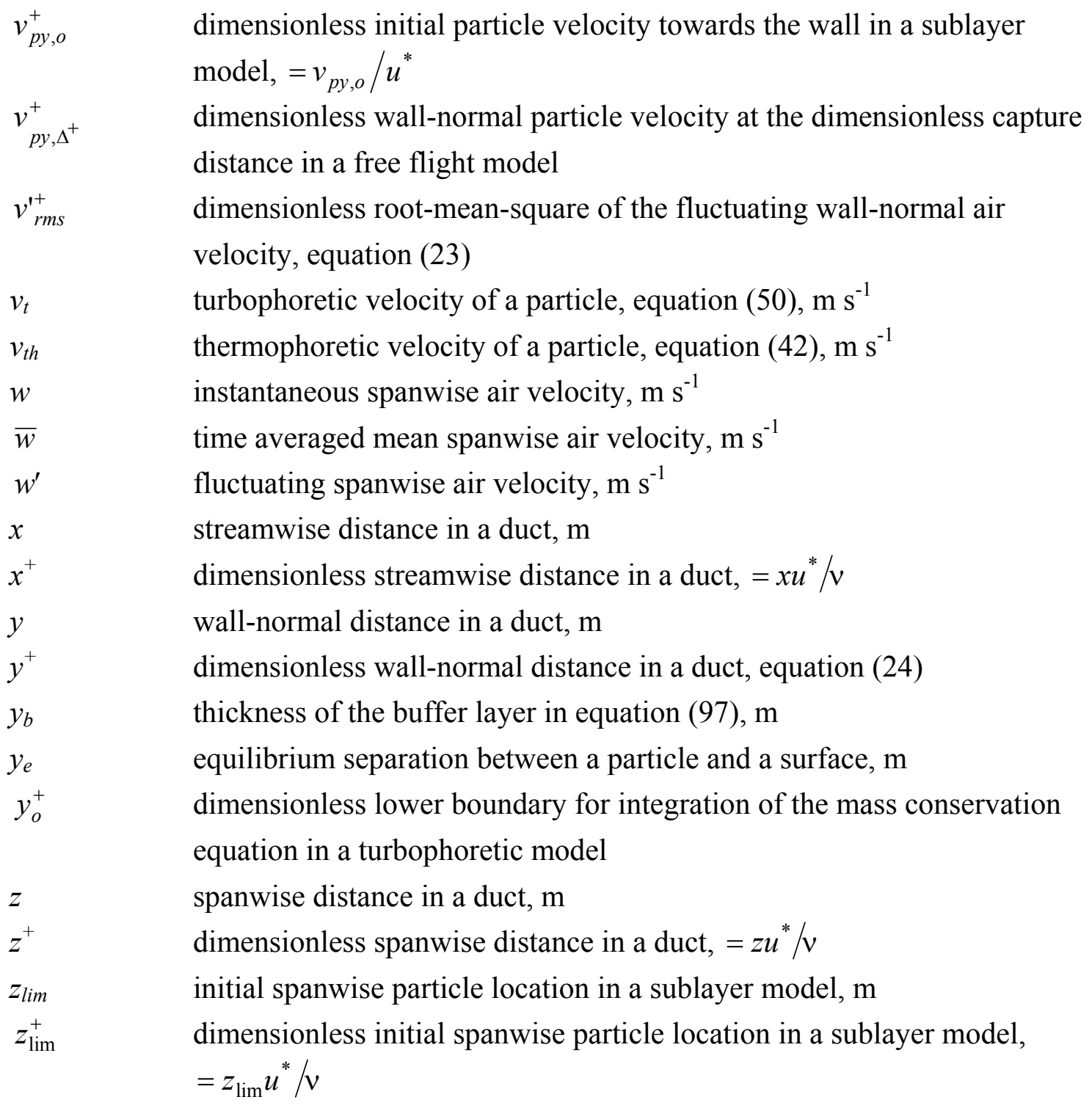

\section{Greek symbols}

$\Delta$

$\Delta^{+}$

$\varepsilon_{o}$

$\phi_{k}$

$\lambda$

$\lambda_{x}$

$\lambda_{x}^{+}$

$\lambda_{y}$ particle capture distance in free-flight deposition models, $\mathrm{m}$ dimensionless particle capture distance in free-flight deposition models permittivity of air, $8.86 \times 10^{-12} \mathrm{C}^{2} \mathrm{~N}^{-1} \mathrm{~m}^{-2}$ roughness parameter in equations (75)-(77) mean free path of gas molecules, $m$ streamwise length of near-wall low-speed streaks, $\mathrm{m}$ dimensionless streamwise length of near-wall low-speed streaks, $=\lambda_{x} u^{*} / v$ wall-normal depth of near-wall low-speed streaks, $\mathrm{m}$ 


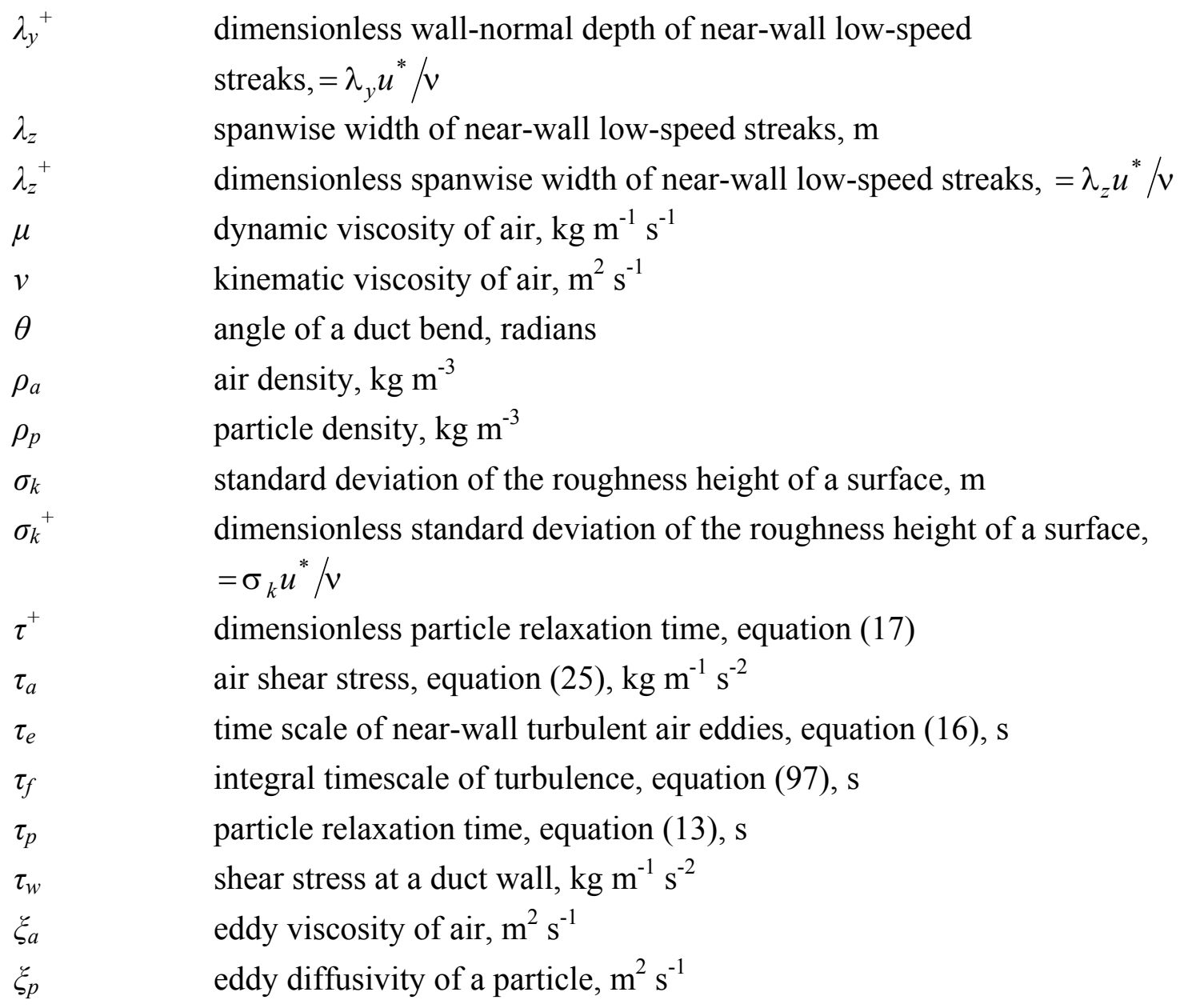




\section{Acknowledgements}

This work was supported by the Office of Nonproliferation Research and Engineering, Chemical and Biological National Security Program, of the National Nuclear Security Administration under U.S. Department of Energy Contract No. DE-AC03-76SF00098. 


\section{Introduction to Particles and HVAC Systems}

This report addresses the issue of particle deposition in heating, ventilation and air conditioning (HVAC) systems of commercial office buildings. Particle deposition throughout all HVAC system components is a broad concern; this report focuses on deposition from turbulent airflow onto duct surfaces.

\subsection{Particles and Human Health}

Particulate matter in air with aerodynamic diameter less then $10 \mu \mathrm{m}\left(\mathrm{PM}_{10}\right)$ is a criteria pollutant regulated by the United States Environmental Protection Agency. In the US, the National Ambient Air Quality Standard (NAAQS) for $\mathrm{PM}_{10}$ is $150 \mu \mathrm{g} / \mathrm{m}^{3}$ averaged

over a 24-hour period and $50 \mu \mathrm{g} / \mathrm{m}^{3}$ averaged over a one-year period. A new standard for particles of aerodynamic diameter less than $2.5 \mu \mathrm{m}\left(\mathrm{PM}_{2.5}\right)$ has been promulgated. It would limit $\mathrm{PM}_{2.5}$ concentrations to $65 \mu \mathrm{g} / \mathrm{m}^{3}$ over a 24 -hour average and $15 \mu \mathrm{g} / \mathrm{m}^{3}$ over a yearly average (http://www.epa.gov/airs/criteria.html). Whether there is any perfectly safe level for human exposure to particulate matter is unknown.

By the start of the 1900's, it was widely acknowledged that air pollution was linked to poor human health. Public attention was more keenly focused on the health impacts of air pollution after episodes of very high particulate matter levels in Meuse Valley, Belgium (December, 1930), Donora, Pennsylvania (October, 1948) and London, England (December, 1952) were observed to be associated with increases in human illness and death. More recent epidemiological studies have demonstrated positive correlations between ambient $\mathrm{PM}_{10}$ concentrations and human morbidity and mortality. Excellent summaries of the findings of these epidemiological studies are available (Pope $\&$ Dockery, 1999; and Pope, 2000). There is also strong epidemiological evidence indicating that ambient $\mathrm{PM}_{2.5}$ contributes to adverse human health effects (Schwartz et al., 1996). This epidemiological evidence suggests that both acute and chronic health effects occur at pollutant concentrations common in US cities and at levels below the NAAQS. Subpopulations most likely to be at greatest risk from $\mathrm{PM}_{10}$ exposure include the elderly, young children, asthmatics and those with preexisting impairment of respiratory and pulmonary systems. While opinions are not unanimous, most epidemiologists and reviewers believe that the body of evidence strongly suggests that exposure to particulate air pollution, and especially $\mathrm{PM}_{2.5}$, is an important risk factor for 
mortality, respiratory symptoms and diseases, and exacerbation of existing pulmonary and cardiovascular diseases.

The link between ambient $\mathrm{PM}_{10}$ concentrations and the concentration to which individuals are exposed has not been fully elucidated. Behavioral studies document that people spend most of their time indoors. Particle concentrations and sources indoors are not the same as outdoors. Most of the air breathed by individuals is indoor air, which raises some questions about the epidemiological link between ambient $\mathrm{PM}_{10}$ concentrations and human health problems. Particle deposition in HVAC systems will influence the concentration of ambient $\mathrm{PM}_{10}$ within a building. Personal exposure concentrations have often been observed to be greater than indoor or outdoor concentrations, possibly due to a 'personal cloud' effect. Personal $\mathrm{PM}_{10}$ exposure concentrations do not correlate well with ambient $\mathrm{PM}_{10}$ levels in cross-sectional studies, but the two measures show a better correlation in longitudinal studies that account for personal variability (Wallace, 2000).

Human exposure to PM has significant associated costs. Total annual cost due to death and morbidity has been estimated at $\$ 28$ billion in the UK (Pearce \& Crowards, 1996) and the benefit in health care savings of achieving new the $\mathrm{PM}_{2.5}$ standards in the US has been estimated at $\$ 32$ billion (Ostro \& Chestnut, 1998).

\subsection{Anti-Terrorism Concerns: Chemical and Biological Agents}

Accidental or intentional airborne release of aerosolized chemical or biological agents within or near a building may lead to exposure of the building occupants to these harmful substances. Agents released outdoors may be drawn into a building by the HVAC system; those released within a building may be spread to other parts of the building by the HVAC system. In either case, deposition in the supply and return ductwork may significantly influence exposures. An understanding of particle deposition in HVAC ducts can also help in planning responses in terms of HVAC system operation in the event of a detected release. Deposition may also be important for post-release remediation since the HVAC system may require decontamination to minimize exposure owing to resuspension of contaminants. 


\subsection{HVAC Systems and Indoor Air Quality}

To understand the contribution of ambient particulate matter to human exposure, it is important to know how the particle size distribution is modified as outdoor air travels into a building. Particle deposition in supply ventilation ducts reduces the indoor concentrations of particles of outdoor origin. Heating, ventilating and air conditioning (HVAC) systems also continuously modify indoor particle concentrations as air is recirculated. Air travels from outdoors into a building via three main routes: mechanical ventilation through a ducted HVAC system, natural ventilation through open doors and windows, and infiltration through gaps and cracks in the building envelope. Most intermediate and large commercial buildings are mechanically ventilated and, for these buildings, mechanical ventilation is usually the dominant entry path of outdoor air to the indoor environment. Consequently, particle deposition in HVAC systems influences particle concentrations within buildings.

HVAC systems consist of all equipment that helps to provide and condition indoor air. This includes louvers, fans, air cleaners, heating and cooling equipment, ducts, humidifiers and dehumidifiers, terminal devices and control equipment. Systems are widely variable in terms of complexity, quality, operation and maintenance. HVAC systems serve the multiple purposes of providing fresh air to the indoor space, controlling indoor air temperature and controlling indoor pollutants by ventilation and, in some cases, building pressurization. The relative humidity of supplied air may also be adjusted to maintain occupant comfort. Standards for acceptable building ventilation and thermal conditions have been established and are maintained by the American Society of Heating, Refrigeration and Air-Conditioning Engineers, as are guidelines for HVAC commissioning and maintenance (ASHRAE, 1989a, 1989b, 1992).

HVAC systems play a central role in maintaining indoor air quality in large buildings and their improper functioning may lead to a variety of problems. Numerous studies have found higher rates of occupant complaints about indoor air quality and health symptoms in mechanically ventilated buildings compared to those that are naturally ventilated (Wargocki et al., 2000). A NIOSH survey found that HVAC deficiencies accounted for more than half of the indoor air quality problems in nonindustrial buildings (Crandall \& Sieber, 1996). Ventilation ducts can act as sinks (and in some cases, sources) for a variety of pollutants including particulate matter, microorganisms and volatile organic compounds (VOCs). Particles may deposit to and resuspend from duct surfaces. Particle 
deposits sorb and desorb VOCs in the passing air stream. Bacteria and fungi deposit on HVAC surfaces and grow if sufficient water is present. Such growth produces microbial VOCs (MVOCs) and may amplify the concentration of bioaerosols in the air stream. Chemical interactions can occur between pollutants and HVAC surfaces, and particle deposits may alter the nature of these surface interactions (Morrison et al., 1998). For example, particles of biological origin often contain unsaturated fatty acids. If these deposit in HVAC ducts, they will be exposed to ozone, which can oxidize the unsaturated acids, producing aldehydes that can be released into the air stream (Pasanen et al., 2000). Deposited materials may also become nutrient sources for microorganisms that release MVOCs. These sorts of transformations might be of great importance in overall HVAC hygiene. In addition to these pollutant interactions, ventilation duct materials like sealants, fibrous insulation and residual manufacturing oils may directly pollute the ventilation air (Batterman \& Burge, 1995). In summary then, particle deposition in HVAC systems alters the exposure of building occupants to particles of outdoor origin and is linked to a host of indoor air quality concerns.

\subsection{Types of HVAC Systems}

One may systems broadly divide HVAC systems into small unitary systems and large central units. Unitary systems provide air to a single building zone, while central units are capable of delivering air to multiple zones with different heating and cooling requirements. Interior portions of large buildings filled with people, lighting and equipment often require cooling even during the coldest months of the year. Perimeter portions of buildings that share walls with the outdoors typically require more flexible temperature control because these areas are more directly influenced by outdoor temperature, wind and direct sunlight.

Unitary systems handle a small flow of air $\left(0.2-2 \mathrm{~m}^{3} / \mathrm{s}\right)$, serve small floor areas $(\sim 150$ $\mathrm{m}^{2}$ ) and have a relatively low initial cost. Multiple unitary systems, each with an independent fan, thermal control and ductwork, may be used to ventilate larger spaces. On average, unitary systems have shorter duct runs than central units because of their decentralized locations. Ducts associated with these systems usually have a hydraulic diameter less than $70 \mathrm{~cm}$ and tend to be constructed of galvanized steel, duct board and flexible duct. Unitary systems are often operated intermittently, under thermostatic control, cycling on and off several times per hour when the building is occupied. Commercial retail strip malls, offices, restaurants and professional buildings are the most 
common buildings in which these systems are found. Such buildings are estimated to make up about half of the non-residential building stock in the United States (Delp et al., 1997).

Central HVAC units serve large building areas (greater than $1000 \mathrm{~m}^{2}$ ) and handle large airflow rates $\left(5-50 \mathrm{~m}^{3} / \mathrm{s}\right)$. Central systems are designed to operate as either constant air volume (CAV) or variable air volume (VAV) systems. Constant air volume systems provide a time invariant flow rate of air to each space, and room temperature is controlled by means of heating or cooling the supplied air. Variable air volume systems achieve temperature control by regulating the amount of cooled air provided to each space. Most central systems feature continuous operation and have galvanized steel ducts of rectangular cross section to distribute the air. Fiberglass lining is commonly used on the interior surface of large ducts near fans to absorb acoustic vibrations and to provide thermal insulation. The plenums and largest ducts in these systems may have a hydraulic diameter of several meters and the smallest ducts, those leading to the room supply registers, have a typical hydraulic diameter of 0.15-0.3 $\mathrm{m}$. Duct air speeds range from maximum of $10-15 \mathrm{~m} / \mathrm{s}$ near the fans to a minimum of $1-2 \mathrm{~m} / \mathrm{s}$ at supply registers. Central systems are sometimes turned off overnight when a building is unoccupied and then operated at higher than normal flow rates in the morning to flush accumulated pollutants from the building before it is reoccupied. Central systems are common in midsized to large office buildings and retail centers, as well as university buildings, theaters and multiple use buildings. Often, several large central systems are required to ventilate very large buildings.

\subsection{HVAC System Components and Particle Deposition}

Figure 1 shows a typical air flow configuration in an HVAC mechanical room. Outside air is brought through louvers into the supply plenum and mixed with a fraction of the return air from the building. This air mixture is filtered, thermally conditioned, and then drawn into a supply fan that distributes the air through a branched duct system to various parts of the building. Return air intakes are located throughout the building. These intakes direct the air through return ducts or plenum spaces back to the HVAC mechanical room where a fraction is recirculated and the rest is exhausted outside the building. 


\subsubsection{Outside air louvers, filters, cooling and heating}

The fraction of outside air in the supply air is controlled by louvers at the air intake and is commonly varied by means of a control system that depends on the outdoor air temperature. Supply air may consist of only outside air if it is at or near the desired temperature. This operation is termed the 'economizer mode' due to the energy savings realized by reducing the need to heat or cool ventilation air. When the outside air temperature deviates from the desired supply temperature, outside air louvers partially close and a larger fraction of return air is directed to the supply. Ventilation standards require that a minimum amount of outside air be brought into any occupied building. Because indoor and outdoor air usually carry different types and concentrations of particulate matter, air louvers influence particle deposition in the rest of the HVAC system by altering the type and amounts of air contaminants introduced into the system.

Filtration in HVAC systems has traditionally been designed to protect mechanical equipment and not human health. Many common HVAC filters are inefficient for particle sizes less than $10 \mu \mathrm{m}$ (Hanley et al., 1994). Filtration efficiency depends on filter type, face velocity and dust loading. Bypass of air around filters has been frequently observed; it has been estimated that $15 \%$ of the provided air does not pass through filters in a typical building (Ottney, 1993). Such filter bypass flow, which could transmit particles of all sizes, is expected to increase as the pressure drop across the filter increases from usage. Return air ducts usually carry unfiltered indoor air that may be contaminated with particles from a variety of indoor sources. Thus, a broad distribution of particle sizes is expected to be present in both supply and return ducts.

Heating or cooling of supply air is usually accomplished by passing air through a fin-andtube type heat exchanger. Such heat exchangers are potentially important sites for particle deposition (Siegel, 2002). They are designed to promote efficient heat exchange, and mass transfer tends to be high in systems with high heat transfer. Fouling induced by particle deposition on heat exchanger surfaces can decrease the effectiveness of heat transfer, degrading the temperature control of the system and increasing the operating cost through the need for a lower temperature coolant (or warmer heating fluid) inside the heat exchanger. In addition, when the supply air is cooled below its dew point, water condenses from the air stream. Condensed water can reduce the size of airflow channels in the heat exchanger and alter particle deposition. If not properly drained, condensed water and the wetted surfaces in the HVAC system can become sites for microbial 
growth. Subsequent release of bioaerosols, such as mold spores, can constitute another source of particles in the ducts.

\subsubsection{Supply fan and ventilation ducts}

After being heated or cooled, the air is distributed through the supply ducts by the supply fan. Particles can deposit on the fan housing and fan blades and, in the case of severe fouling, impede its performance. As particles deposit on filters, heat exchangers and ducts, the resistance to airflow through these systems may increase. For a constant air supply rate, increasing the airflow resistance increases the pressure drop along the duct, causing the fan to consume more energy; the magnitude of this increased energy consumption depends on the specific performance conditions of the fan.

Increasing the flow resistance can also reduce the rate at which air is delivered to the indoor space. Such a reduction in flow rate commonly leads to decreased fan energy use (Parker et al., 1997). Particle deposits that alter the airflow and pressure drop in a duct system will also influence the duct leakage rate and the overall rate of energy use by the HVAC system through the rate at which energy is lost by conduction through duct walls. Thus, the effect of particle deposits on overall HVAC system energy consumption is uncertain in both sign and magnitude and is likely to be HVAC system dependent.

Ventilation duct systems usually consist of a very large duct after the supply fan that branches several times into successively smaller diameter ducts to deliver air to a variety of locations within the building. Duct branches, bends and reducing sections are required to achieve proper air distribution and maintain air velocities. Most ducts are fabricated from sheet metal, but the smallest ducts that lead to supply registers are often made of flexible aluminum or Mylar to allow for easier installation. A length of duct is made up of several short sections connected in series by various fittings. These fittings can serve as sites for local particle deposition. Air may leak through the joints between duct sections and through seams resulting from duct fabrication. Studies of duct leakage in California buildings have found average leakage rates in supply ducts as a percentage of the system flow rate at the inlet to be $25 \%$ in light commercial buildings (Levinson et al., 1997) and 10-20\% in large commercial buildings (Fisk et al., 1999). Particles will exit ducts with leakage air in positively pressured (supply) ducts and enter ducts through leaks in negatively pressured (return) ducts. 
Ideally, duct surfaces should be kept clean and dry; however, even new ducts may be soiled from storage prior to installation and debris from the building's construction phase. In addition, new steel ducts have been identified as sources of VOCs in indoor air from residual oils left from the original machining and fabrication (Pasanen et al., 1995).

Airflow through ventilation ducts is turbulent, and particles can deposit on ducts owing to interactions with this turbulence, by gravitational settling, and by other mechanisms. With usage, ducts have been observed to accumulate particulate deposits on their interior surfaces. Wallin (1994) observed that such deposits could reduce the amount of air flowing through ducts, especially small diameter ducts, and thus could degrade the performance of the ventilation system. Previous measurements of the density of dust deposits on the floors of ventilation ducts in office buildings and schools and the inferred dust accumulation rates are presented in Table 1. General consistency among studies is observed for the mean and range of both the deposit density and accumulation rates, despite variability in the methods used and in the building location and age. The size distributions of such deposited dusts have not been measured, but the total mass of the deposits in all cited studies is likely to have been dominated by very large particles, debris and fibers. Measurement of deposit density has been shown to vary depending on the method used for quantification (Holopainen et al., 1999).

Duct cleaning is an increasingly common practice in both residential and commercial buildings. It can help maintain proper duct flow rates and provide a potential preventive and corrective benefit for indoor air quality. Duct cleaning businesses in the US are certified by the National Air Duct Cleaners Association (NADCA), which has developed standards and methods for duct cleaning and cleanliness measurement. The maximum deposit density for a duct to be considered clean is $0.1 \mathrm{~g} / \mathrm{m}^{2}$ based on a vacuum-andfilter-cassette method (NADCA, 1992).

\subsubsection{Duct components and terminal devices}

Ventilation systems include duct components that locally modify airflow and offer surface area for particle deposition. Fin-and-tube heating and cooling coils are often installed at the end of the ducted distribution system to allow occupants local thermostatic control of air temperature. Turning vanes, dampers, variable air volume boxes and registers help direct the air stream, control flow rates and distribute air 
properly. The presence of such components can alter the fate of particles that enter HVAC systems.

\subsubsection{Summary}

The proper functioning of HVAC systems in mechanically ventilated buildings is important for maintaining indoor air quality. Particle deposits can detrimentally affect HVAC systems in a variety of ways such as reducing air flow rates, fostering biological growth and emitting VOCs. Deposition can also increase or decrease overall HVAC system energy consumption depending on its specific operating conditions. There is also the potential for particle deposits to become modified and then become secondary sources of pollution. Particle deposition in HVAC systems reduces the exposure of building occupants to particles of outdoor origin. HVAC systems also continuously modify exposure to indoor particles as air is recirculated. 


\section{Experimental Work on Particle Deposition from Turbulent Tube and Duct Flows}

There is widespread interest in the deposition of particles from turbulent airflows due to its applicability in such diverse fields as aerosol sampling, inhalation toxicology, atmospheric transport and fate of pollutants, air cleaning and semiconductor microcontamination. Numerous reviews of turbulent particle deposition experiments and theories exist (Kneen \& Strauss, 1969; Owen, 1969; Sehmel, 1980; Papavergos \& Hedley, 1984); however, several advances in understanding this phenomenon have been made since the most recent summary. Investigations into this topic in the literature have utilized three main methods: physical experiments, Eulerian modeling, and Lagrangian simulations.

Section 2 reviews published experimental investigations of particle deposition rates from turbulent flows. This section begins by defining parameters useful for discussing particle deposition from turbulent flows. Subsequently, experimental studies of particle deposition in straight tubes and ducts are discussed, then experiments performed in tube bends are considered. The relevance of the overall data set to the case of particle deposition in ventilation ducts is discussed in the last part of this section. The experimental review is limited to investigations in which sufficient information was reported so that specific deposition rates could be associated with specific particle sizes for a given deposition surface. Turbulent duct flows are discussed in section 3. Empirical equations, Eulerian models and Lagrangian simulations predicting particle deposition rates from turbulent flows are discussed in Section 4.

\subsection{Definition of Parameters}

The deposition velocity, $V_{d}$, of a particle to a duct surface is defined as

$$
V_{d}=\frac{J}{C_{a v e}}
$$

where $J$ is the time-averaged particle flux to the surface (mass or number per area per time) and $C_{a v e}$ is the time-averaged airborne particle concentration in the duct (mass or number per volume), usually evaluated at the centerline of the flow. Among other factors, the deposition velocity is a function of particle size. 
Penetration through a duct is defined by

$$
P_{\text {duct }}=\frac{C_{\text {outlet }}}{C_{\text {inlet }}}
$$

where $C_{\text {outlet }}$ and $C_{\text {inlet }}$ are the flow-weighted average particle concentrations at the outlet and inlet of the duct, respectively. If the deposition velocity is known for a given particle size, and deposition to the duct interior is uniform (or the deposition velocity is interpreted as the area-weighted average over all surfaces), then the penetration of that particle size through a straight duct section is related to the deposition velocity as follows:

$$
P_{d u c t}=\exp \left(\frac{-4 L V_{d}}{D_{h} U_{\text {ave }}}\right)
$$

where $L$ is the duct length, $U_{\text {ave }}$ is the bulk average air speed in the duct and $D_{h}$ is the hydraulic diameter of the duct defined as

$$
D_{h}=\frac{4 A}{P}
$$

Here, $A$ is the cross sectional area of the duct and $P$ is the perimeter of a section through the duct, normal to the direction of flow.

Turbulent duct flows can be characterized in part by their turbulence intensity as measured by the friction velocity, $u^{*}$, which is defined as

$$
u^{*}=\sqrt{\tau_{w} / \rho_{a}}
$$

where $\tau_{w}$ is the shear stress at the duct wall and $\rho_{a}$ is the air density. Assuming that the wall shear stress is uniform inside the duct, a balance of pressure forces and shear stress forces in the duct leads to this relationship:

$$
u^{*}=U_{\text {ave }} \sqrt{f / 2}
$$

where $f$ is the Fanning friction factor. For a fully developed turbulent flow, $f$ is given by

$$
f=\frac{\Delta P}{\Delta L} \frac{D_{h}}{2 \rho_{a} U_{a v e}^{2}}
$$


where $\Delta P / \Delta L$ the pressure drop per unit duct length. The friction velocity may be determined experimentally for a known air velocity and hydraulic duct diameter, by means of measuring the pressure drop along the duct, and by applying equations (6) and (7). Alternatively, empirical expressions may be used to calculate the friction factor. For turbulent flows and smooth walls, the Blasius law or the von Karman correlation may be used to estimate the friction factor.

$$
\begin{array}{lll}
\text { Blasius: } & f=0.0791 \cdot \operatorname{Re}^{-0.25} & \left(2800<\operatorname{Re}<10^{5}\right) \\
\text { von Karman: } & 1 / \sqrt{f}=4.0 \log (\operatorname{Re} \sqrt{f})-0.4 & \left(2800<\operatorname{Re}<3.2 \times 10^{6}\right)
\end{array}
$$

Here, Re is the Reynolds number of the duct flow, based on the average flow velocity and the hydraulic diameter of the duct:

$$
\operatorname{Re}=\frac{D_{h} U_{\text {ave }}}{v}
$$

where $v$ is the kinematic viscosity of the air. For turbulent flow past smooth walls, the friction at the surface results from viscous drag. For rough walls, form drag on the roughness elements may be an important contributor to the total friction. If the mean microscale roughness height is $k$, the dimensionless roughness height (also known as the roughness Reynolds number) can be defined by

$$
k^{+}=\frac{k u^{*}}{v}
$$

Schlichting (1979) defined three regimes of flow resistance for turbulent flow in rough pipes:

$$
\begin{array}{ll}
\text { hydraulically smooth regime: } & k^{+} \leq 5 \\
\text { transition regime: } & 5 \leq k^{+} \leq 70 \\
\text { completely rough regime: } & k^{+}>70
\end{array}
$$

In the hydraulically smooth regime, roughness elements are submerged in the nearly laminar layer near the wall and roughness does not significantly influence the friction of the flow. In this case, the friction factor depends only on viscosity (through $R e$ ) as seen in equations (8) and (9). As $k^{+}$grows to greater than 5, a portion of the roughness elements protrude into more turbulent flow and form drag on these elements increases the flow resistance relative to a smooth wall. In this case, the friction factor is observed to depend on both the air viscosity (through $\mathrm{Re}$ ) and on the relative roughness height, $k / D_{H}$. In the completely rough regime, roughness elements protrude far into the turbulent flow 
and dominate the flow resistance so that the friction factor no longer depends significantly on $\mathrm{Re}$, rather it depends on the relative roughness height alone. Equations for computing the friction factor of flows through ducts with rough walls are provided in Table 2. These equations strictly hold only for closely-packed, sand-grain type roughness elements. For differently shaped or more widely spaced roughness, an equivalent sand grain roughness can be defined, but it must be determined experimentally. For very large roughness elements like grasses, friction velocities are usually determined based on measurements of the velocity profile far from the wall, in the logarithmically varying region of the flow.

The friction velocities in a smooth-walled ducts is typically of the order of about $5 \%$ of the average velocity; in rough walled ducts, the friction velocity is a slightly greater proportion of the mean flow. The range of friction velocities expected in ventilation ducts is about $0.1-1.0 \mathrm{~m} / \mathrm{s}$. In terms of flow resistance, most clean steel ducts are expected to be in the hydraulically smooth regime. Ducts with roughness caused by deposits, corrosion or insulation are likely to be either in the hydraulically smooth regime or the transition regime, although the completely rough regime may be approached in some cases with large roughness elements and high friction velocities. The equivalent hydraulic roughness of fiberglass duct insulation has been estimated to be $3.0 \mathrm{~mm}$, meaning most flows through insulated ducts are expected to be in the transitional or completely rough regime (ASHRAE, 1995).

The dimensionless particle deposition velocity is defined by normalizing the dimensional deposition velocity with the friction velocity:

$$
V_{d}^{+}=V_{d} / u^{*}
$$

In studies of particle deposition from turbulent flow, it is common to investigate the relationship between the dimensionless particle deposition velocity and the dimensionless particle relaxation time. The dimensional relaxation time of a particle, $\tau_{p}$, is the characteristic time for a particle velocity to respond to a change in air velocity. It may be calculated for particles in the Stokes flow regime as follows

$$
\tau_{p}=\frac{C_{c} \rho_{p} d_{p}^{2}}{18 \mu}
$$


where $C_{c}$ is the Cunningham slip correction factor, $\rho_{p}$ is the particle density, $d_{p}$ is the particle diameter $\mu$ is the dynamic viscosity of air. The slip correction factor can be estimated by the expression

$$
C_{c}=1+\operatorname{Kn}\left[1.257+0.4 \exp \left(-\frac{1.1}{\mathrm{Kn}}\right)\right]
$$

where the Knudsen number, $\mathrm{Kn}$, is

$$
\mathrm{Kn}=\frac{2 \lambda}{d_{p}}
$$

and $\lambda$ is the mean free path of gas molecules, equal to $0.065 \mu \mathrm{m}$ at a temperature of $25{ }^{\circ} \mathrm{C}$ and atmospheric pressure.

Turbulent eddies in duct flows display a wide range of length scales, with the largest eddies limited by the transverse duct dimensions and the smallest limited by the dissipative action of molecular viscosity. Smaller eddies tend to be shorter lived while larger eddies persist for a longer time before disappearing. The smallest eddies in a flow are those near the walls and their average lifetime may be estimated by

$$
\tau_{e}=v / u^{*^{2}}
$$

Because deposition happens at walls, particle interactions with near-wall eddies are potentially important in determining deposition rates. A dimensionless particle relaxation time, $\tau^{+}$, can be defined by comparing the particle relaxation time to the timescale associated with the near-wall turbulent eddies

$$
\tau^{+}=\frac{\tau_{p}}{\tau_{e}}=\frac{C_{c} \rho_{p} d_{p}^{2} u^{* 2}}{18 \mu \nu}
$$

In general, particle motion is only affected by eddies with duration at least as long (in a magnitude sense) as the particle relaxation time. Particles do not have sufficient time to respond to the shorter lived eddies. A value of $\tau^{+}<0.1$, indicates that a particle is able to fully respond to even the smallest turbulent eddies. In this case, the particle is expected to closely follow all turbulent air fluctuations. A particle with $\tau^{+}>10$ will be relatively unaffected by the small near-wall eddies and will only be significantly affected by larger eddies further from the wall. The motion of particles with relaxation times close to the 
lifetime of the near-wall eddies, $0.1<\tau^{+}<10$, is expected to be heavily influenced by these eddies, with instantaneous particle velocities equilibrating with, but then disengaging from, the local air velocity. Consequently, such particles frequently shoot ahead of or lag behind the near wall eddies.

Considering duct flows with $u^{*}=0.1-1 \mathrm{~m} / \mathrm{s}$ and particles in the diameter range 0.003-30 $\mu \mathrm{m}$, expected values of $\tau^{+}$span from about $10^{-6}$ for a $0.003 \mu \mathrm{m}$ particle in a flow with low turbulence, up to about 100 for a $30 \mu \mathrm{m}$ particle in a highly turbulent flow.

For particle deposition to smooth, vertical walls, the dimensionless deposition velocity is expected to be a nearly unique function of the dimensionless relaxation time. In the case of rough deposition surfaces, the dimensionless deposition velocity can also be strongly influenced by the shape and magnitude of the roughness elements. Electrical and thermal forces can also significantly influence the dimensionless deposition velocity, as can gravitational forces in the case of a non-vertical deposition surface.

\subsection{Experimental Data from Straight Tubes and Ducts}

Many experimental investigations have been conducted that pertain to particle deposition from turbulent airflow through ducts. Major factors that have been observed experimentally to influence particle deposition rate include particle size, degree of air turbulence, surface orientation with respect to gravity and roughness of the deposition surface. The best experimental investigations are those that employ a highly monodisperse aerosol, have a well characterized air flow and deposition surface, and directly measure deposited particles on the wall surface. These conditions are satisfied by only a small fraction of the studies.

Experimentally measured particle deposition velocities from turbulent flows have historically been presented as plots of $V_{d}^{+}$versus $\tau^{+}$. Figure 2 is such a plot showing most of the published data for particles depositing from flow through vertically oriented tubes of small diameter. This figure illustrates the importance of particle size, as measured by $\tau^{+}$, in determining particle deposition. The data of Shimada et al. (1993) for deposition from a horizontal tube is included so as to extend the lower range of $\tau^{+}$and to illustrate the trends in deposition as $\tau^{+}$becomes very small. (The effect of gravity on deposition from this horizontal flow is expected to be negligible owing to the very small particle sizes, $d_{p}=0.01-0.04 \mu \mathrm{m}$, used in these experiments.) 
Following the terminology of Wood (1981b), the data in Figure 2 are divided into three regimes: the diffusion regime, the diffusion-impaction regime, and the inertia-moderated regime. Although the data are broadly scattered in this plot, trends are still clearly visible. In the diffusion regime, particles have small inertia and so follow all turbulent eddies. Particle transport to surfaces depends mostly on Brownian and turbulent diffusion. In a typical HVAC duct flow, turbulent diffusion is much stronger than Brownian diffusion, except extremely close to the duct wall where turbulent fluctuations decay to zero owing to the no-slip boundary condition and the impermeable wall. The dimensionless deposition velocity decreases as $\tau^{+}$increases for $\tau^{+}<0.1$ because of the decrease in Brownian diffusivity as particle size increases. In the diffusion-impaction regime, particles follow turbulent air fluctuations less faithfully and may shoot ahead of or lag behind eddies near the wall. Hence, through this interaction between particle inertia and turbulent eddies, particles may deposit without relying on Brownian diffusion to make the final step to the surface, and $V_{d}{ }^{+}$increases substantially even for relatively small increases in $\tau^{+}$. For the largest particles, those in the inertia-moderated regime, the dimensionless deposition velocity is observed to level off to become nearly independent of $\tau^{+}$. In this case, particles are too large to respond to the rapid fluctuations of near wall eddies and transport to the wall by turbulent diffusion is very weak. These particles reach the wall through momentum imparted by large eddies in the core of the turbulent flow.

The wide scatter among the data in Figure 2 is in part a testament to the difficulty of obtaining accurate experimental data in even the simplest turbulent flow. In the diffusion-impaction regime particle deposition scales roughly in proportion to $d_{p}{ }^{4}$. Thus, small errors in particle sizing or small amounts of polydispersity in the aerosol can lead to large errors in properly interpreting the experimental results. In addition, the character of the deposition surface can significantly influence particle deposition velocities. Roughness, even on the scale of a few microns, may have a big effect on deposition. Unfortunately, the roughness of deposition surfaces has rarely been measured and reported in experimental studies. Other factors that may contribute to the data scatter are differences in particle density, differences in method and data quality from different investigators and differences in the magnitude of the lift force between vertical upward flow and downward flow (Fan \& Ahmadi, 1993). Thermophoretic and electrophoretic forces may also influence particle behavior unbeknownst to the investigator. Furthermore, particle bounce or resuspension have occasionally been observed in some investigations and accounting for these processes is difficult. 


\subsection{Details about Experiments in Straight Tubes and Ducts}

Tables 3-7 summarize the characteristics of the ducts, particles and methods used in most of the published experimental studies on aerosol deposition from turbulent duct flows. Tables 3 and 4 respectively summarize experiments conducted in horizontal and vertical tubes with hydraulic diameters less than $2.7 \mathrm{~cm}$. Tables 5 and 6 respectively summarize studies in horizontal and vertical tubes with hydraulic diameters greater than $2.7 \mathrm{~cm}$. Table 7 explains the codes used to describe the experimental methods in the columns labeled 'Methods \& Comments' in Tables 3-6.

Methods for experimentally determining particle deposition velocities have varied widely, but two broad schemes are available. The first method involves direct measurement of the airborne concentration and the particle flux to the surface followed by calculation of the deposition velocity according to equation (1). Methods $a, b$ and $c$ in the column entitled 'Methods \& Comments' in Tables 3-6 are variations of this technique. The principal alternative method is to measure particle air concentrations in the duct at upstream and downstream locations and infer the deposition velocity by equations (2) and (3). This is method $d$ in Tables 3-6. The fluorometric, radioactive and microscopic techniques of methods $a-c$ are much more sensitive and reliable for determination of deposition velocities than method $d$. When using method $d$, small errors in measuring penetration can lead to large errors in calculating the deposition velocity, especially for particle penetrations near zero or 1. Also, particle loss mechanisms other than deposition to duct walls may influence the interpretation of data collected by method $d$. Those methods in which deposition flux is measured directly are far less susceptible to these types of errors. Data collected by method $d$ often exhibit low reproducibility (e.g., Lee \& Gieseke, 1994) and are rarely of high quality.

Measurement of airborne concentration in duct flows usually involves filter sampling or quantification by a particle counting device, though alternative techniques (methods $w, x$ and $z$ in Tables 3-6) have been implemented. As a rule, isokinetic sampling should be utilized to deliver a representative sample to the filter or particle counter, especially for particles larger than $2 \mu \mathrm{m}$. Often in these experiments, the ducts were too small to accommodate a sampling probe and the entire air stream was sampled to determine the concentration. Uncertainties in concentration measurements may arise from anisokinetic sampling, deposition losses in sampling lines, intrinsic uncertainties of particle 
monitoring equipment and laboratory analytical errors. Isokinetic sampling or absolute filtration coupled with fluorometric or radioactive techniques (methods $t$ and $u$ ) are likely to yield the most reliable results. Microscopic counting of filter samples may yield high quality results as well, but is likely to be more susceptible to errors by the investigator. Where fluorometric, radioactive or microscopic techniques were used for surface flux determination, the same technique was used for analysis of filter samples.

Concentrations measured by particle counters generally have a larger uncertainty than filter samples owing to variations in the performance of the device, the increased potential for particle loss in sampling lines and the difficulty of achieving isokinetic sampling with a constant flow pump.

Accurate determination of the diameter of experimental particles is important. Dimensionless relaxation time is proportional to the square of the particle diameter, and $V_{d}{ }^{+}$is observed to be approximately proportional to the fourth power of particle diameter in the diffusion-impaction regime. Thus, small errors in sizing can thus translate into large errors in the determination of deposition rates. Comments labeled $f-m$ in the tables refer to the method of particle sizing used in a particular experiment.

The duct material, duct diameter, roughness, and flow orientation all play a role in determining particle deposition from a duct flow. A horizontal rectangular duct has three distinct internal surfaces: the floor (upward facing), the wall (vertically oriented) and the ceiling (downward facing). For particles larger than roughly $0.1 \mu \mathrm{m}$ diameter, deposition velocities to these surfaces are expected to differ owing to the influence of gravity. Particles in horizontal ducts of round cross section are expected to deposit in a similarly nonuniform manner as in rectangular ducts due to gravity. In the case of a vertical round duct, all duct surfaces are the same and deposition is expected to be uniform over the entire internal duct circumference. Nearly all investigations in horizontal flows have examined deposition only to the duct floor; only Sehmel (1973) looked at differences in deposition to the duct floor and ceiling. In cases where deposition velocities are inferred from concentration measurements (Adam et al., 1996; Cheong, 1997), the deposition surface must be considered to include the entire internal perimeter of the duct and information on differences in deposition to distinct surfaces is inaccessible. Deposition surface materials have included a variety of metals and plastics, as well as glass. Strong electrostatic effects may influence particle deposition when the deposition surface is nonmetallic, especially when steps have not been taken to neutralize the test aerosol (comment $n$ in Tables 3-6). 
Duct roughness may be divided into microscale roughness, with average roughness heights much less than a millimeter, and macroscale roughness, referring to roughness elements on the order of a millimeter or larger. Theoretical (Browne, 1974; Wood, 1981 a; Fan \& Ahmadi, 1993; Li et al., 1994) and experimental (El-Shobokshy, 1983; Wells \& Chamberlain, 1967; Lai, 1997) evidence suggests that both roughness scales influence particle deposition. All real materials possess microscale roughness, and this type of roughness has rarely been quantified in experiments. Ducts with significant microscale roughness are often hydraulically smooth, meaning that the friction factors of flows in the duct are the same as if the duct walls were perfectly smooth. Because Brownian diffusivities of particles are much smaller than the molecular viscosity of air, the particle boundary layer is much thinner than the aerodynamic boundary layer. Therefore, microscale roughness elements can enhance particle deposition even in hydraulically smooth flows and, when particle deposition is the concern, more precise measurement of the roughness is needed than simple classification of the surface as hydraulically smooth. Macroscale roughness, in the form of fibers or grasses (Chamberlain, 1967; Wells \& Chamberlain, 1967; Sehmel, 1970a), repeated ribs (Chamberlain et al., 1984; Hahn et al., 1985; Lai, 1997) and uniform three-dimensional elements (Lai, 1997), has been more frequently characterized and its influence on deposition more systematically investigated. Roughnesses reported in Tables 3-6 include both microscale and macroscale conditions.

On occasion, researchers have seen fit to apply a coating of petroleum jelly, viscous oil or similar tacky substance to the deposition surface to prevent particles from bouncing upon impact or from becoming reentrained into the flow after depositing. These surface treatments have allowed some researchers to assess the role of particle bounce or reentrainment in their experiments. The way such coatings may influence particle deposition from the standpoint of microscale roughness is not apparent. Evidence of particle bounce or reentrainment has been observed in some experiments (Friedlander \& Johnstone, 1957; Postma \& Schwendiman, 1960; Chamberlain, 1967; Rouhiainen \& Stachiewicz, 1970). When observed, these phenomena have been greater for larger particle sizes and higher flow rates.

Deposition of liquid droplets in annular flow has been frequently considered experimentally. Annular flow consists of a thin liquid layer on the walls of a conduit flowing concurrently with the air stream. Disturbances at the liquid-air interface cause 
polydisperse droplets to be released into the air and it is the deposition of these droplets back into the liquid layer that is studied. A review of experiments of droplet deposition from annular flow is available (McCoy \& Hanratty, 1977). Investigations of this type were not included in this paper because of the polydisperse nature of the aerosol and the poorly characterized wave-like surface condition at the liquid-air interface.

\subsection{Historical Development of Experiments in Straight Tubes \& Ducts}

\subsubsection{Particle size and air velocity}

The seminal experimental investigation into understanding particle deposition from turbulent flows was conducted by Friedlander \& Johnstone (1957). Their data showed increased particle deposition with increases in air velocity and particle diameter for particles in the diffusion-impaction regime. Subsequent measurements of deposition from small diameter tubes have confirmed these findings (Postma \& Schwendiman, 1960; Sehmel, 1968; Liu \& Agarwal, 1974).

Using glass tubes, Liu \& Agarwal (1974) conducted experiments that are widely considered the benchmark for particle deposition from turbulent flow due to the high quality of their methods and the reproducibility of the data. The data are shown in Figure 3 along with those of El-Shobokshy (1983) and are observed to cover both the diffusionimpaction and inertia moderated regimes. The data of Liu \& Agarwal clearly show the large increase in deposition velocity with particle size in the diffusion-impaction regime and a subtle decrease in deposition velocity as particle size increases in the inertiamoderated regime. This leveling of dimensionless deposition velocities for large values of $\tau^{+}$has been corroborated experimentally by Forney \& Spielman (1974).

Wells \& Chamberlain (1967) were the first to investigate deposition of particles to smooth surfaces in the diffusion regime. Their data confirmed the decrease in deposition as particle size increases within the diffusion regime to the point where Brownian diffusion becomes negligible. Chamberlain et al. (1984) made a single observation of deposition in the diffusion regime. Shimada et al. (1993) provided a larger data set of reasonable quality for deposition in the diffusion regime, showing increasing deposition for decreasing particle size. The data of Wells \& Chamberlain and Shimada et al. are displayed in Figure 2. 


\subsubsection{Microscale roughness}

El-Shobokshy (1983) explored deposition to surfaces with three different levels of microscale roughness: smooth glass and brass machined to microscale roughnesses of 7 and $20 \mu \mathrm{m}\left(k^{+}=0.5\right.$ and 1.5). As shown in Figure 3, his data agree well with those of Liu $\&$ Agarwal (1974) for the case of the smooth surface. Deposition to the roughened surfaces was enhanced up to two orders of magnitude as compared to the smooth case. The deposition enhancement was greatest for the smallest particles studied. To date, this is the only experimental investigation to systematically investigate the connection between increases in microscale roughness and increases in particle deposition velocities in the diffusion-impaction regime.

It should also be noted that Postma \& Schwendiman (1960) saw either no difference in deposition or slightly less deposition to 'as fabricated' and 'grit roughened' surfaces as compared to polished tubes in the diffusion-impaction regime. Sehmel (1968) classified some pipes in his study as smooth or rough based on a visual inspection. In the two reported experimental runs comparing smooth and rough pipes, the deposition rate to the rough pipe was larger than to the smooth pipe in one case, and the deposition rates were equal in the other case. Ilori (1971) estimated the size of roughness elements in his experimental tubes by examining the surfaces under a microscope. The glass tube was determined to be optically smooth, while the plastic and aluminum tubes were found to have maximum roughness heights of about $5 \mu \mathrm{m}$. Particles in the diffusion-impaction regime had measured deposition rates to the rough surfaces that were equal to or up to two times greater than those to the glass surface. It seems likely that microscale roughness enhances particle deposition for some particle sizes, and the systematic inquiry by El-Shobokshy supports this viewpoint; however, investigations with more qualitative assessments of surface roughness have found it to have much less of an impact on particle deposition than indicated by the measurements of El-Shobokshy.

\subsubsection{Fibrous and macroscale roughness}

Wells \& Chamberlain (1967) studied particles in the diffusion and diffusion-impaction regimes depositing to a hydraulically smooth brass surface and a surface with fibrous roughness elements with an average length of about $100 \mu \mathrm{m}$. The results of these experiments are shown in Figure 4, where it can be seen that particle deposition to the 
fibrous roughness was measured to be up to 3 orders of magnitude greater than deposition to the smooth brass surface.

Sehmel (1970a) investigated deposition of 6-14 $\mu \mathrm{m}$ particles to artificial grass on the floor of a square duct. The results of this study are presented in Figure 5, along with later results (Sehmel, 1973) showing deposition to a smooth surface on the floor of the same duct. Lines predicting particle deposition by gravitational settling to a smooth floor surface at the three friction velocities of Sehmel's 1973 experiments are also included in the figure. Dimensionless deposition velocities are seen to be approximately equal for both surfaces for larger particles. On the other hand, deposition to the artificial grass surface is enhanced by about an order of magnitude over the smooth surface as particle diameter decreases. This is the same trend as observed by El-Shobokshy (1983) for deposition to microscale roughness.

Lai (1997) measured dimensionless deposition velocities of $0.7-7.1 \mu \mathrm{m}$ particles to surfaces with two-dimensional rib roughness and three-dimensional blocks on the floor of a duct and compared these values to those for deposition onto the smooth floor of the duct. Compared to smooth surfaces, deposition velocities to surfaces with twodimensional ribs were observed to be 2-3 times higher and those to surfaces with threedimensional blocks higher by a factor of 5-19. In addition, the distribution of threedimensional roughness blocks on the surface was found to slightly influence measured particle deposition. Chamberlain et al. (1984) studied the effects of two-dimensional rib type roughness on the deposition of large particles and Hahn et al. (1985) performed similar experiments for small, diffusive particles. Effects of spacing between the ribs were unclear in both cases and neither work offered a comparison of deposition to a comparable smooth surface.

\subsection{Particle Deposition in Tube Bends}

Deposition in bend sections is potentially important, but it has rarely been investigated experimentally under turbulent flow conditions. Only two experimental inquiries have been conducted on aerosol deposition in bends with turbulent flow and these were both conducted in very small diameter tubes. The conditions of these experiments are summarized in Table 8. The interior surface roughness of the deposition tube was not reported in either experiment. The bend ratio, $R_{o}$, presented in the table is the ratio of the bend radius to the hydraulic radius of the tube 


$$
R_{o}=\frac{2 R_{\text {bend }}}{D_{h}}
$$

where $R_{\text {bend }}$ is the radius of the bend measured at the centerline.

Pui et al. (1987) investigated particle penetration through 90-degree bends in both laminar and turbulent flow conditions, but only the turbulent flow results are discussed here. McFarland et al. (1997) conducted experiments similar to those of Pui et al. in 90degree bends with a variety of bend ratios. These investigators found it most appropriate to present their data as plots of bend penetration versus particle Stokes number as shown in Figure 6. The Stokes number is defined by

$$
\mathrm{St}=\frac{C_{c} \rho_{p} d_{p}^{2} U_{a v e}}{9 \mu D_{h}}
$$

The experimental data show a sharp decrease in bend penetration as the Stokes number increases from near zero to 1 . The data of McFarland et al. in Figure 6 clearly show that increasing the bend ratio tends to increase particle penetration through the bend for a given value of the Stokes number, especially for bend ratios in the range of 1-4. The data collected by Pui et al. at a bend ratio of 5.7 show good agreement with the trends in the data of McFarland et al., but the data of Pui et al. suggest somewhat greater penetration at the higher Stokes numbers.

Figure 7 shows the data of Pui et al. (1987) and McFarland et al. (1997) in a plot of $V_{d}^{+}$ versus $\tau^{+}$along with the experimental data of Liu \& Agarwal (1974) for particle deposition in a straight tube collected under comparable conditions. Dimensionless deposition velocities in these bends are observed to be greater than those in the straight tube sections, in some cases by 1-2 orders of magnitude.

The deposition velocities in these experiments in tube bends are quite high and are potentially important in terms of particle penetration through HVAC ducts. However, the tubes in which these experiments were conducted were of very small diameter and their relevance to the much larger diameter ducts in ventilation systems is uncertain. 


\subsection{Relevance of Current Data to Deposition in Ventilation Ducts}

Most of the experimental work conducted to date is not directly applicable to the case of particle deposition from flow through HVAC ducts. Several studies have focused on particle losses in aerosol sampling lines and have been performed in small diameter tubes with very high average air velocities and friction velocities. Experiments in ducts with hydraulic diameters of $15 \mathrm{~cm}$ or larger, similar to those in HVAC systems, have often focused on very large grass-like or ribbed roughness not commonly found in ventilation ducts. However, the experimental information gathered to date does provide a mostly consistent picture that can lead to informed expectations of particle behavior in HVAC systems. The overall data set places bounds on the expected deposition behavior of particles in ventilation ducts and provides a foundation for understanding upon which more detailed questions about particle behavior in turbulent flows may be investigated.

The data of Liu \& Agarwal (1974) collected in vertical tubes have proven valuable for evaluating the predictive capabilities of theoretical models and provide an estimate of the magnitude of deposition that can be expected to occur on vertical surfaces in HVAC systems. The finding by Wells \& Chamberlain (1967) of an increase in particle deposition to fibrous filter paper by orders of magnitude as compared to smooth brass leads to questions regarding the analogous situation of deposition to fiberglass liners on duct interiors compared to galvanized steel. The increases in particle deposition velocities with increases in microscale roughness in the experiments of El-Shobokshy (1983) indicate that deposition in ducts may increase as duct surfaces corrode and scale or as particles deposit to surfaces and thereby contribute to microscale surface roughness. The deposition enhancement on two-dimensional rib roughness compared to a smooth surface measured by Lai (1997) informs expectations about particle deposition at joints between duct sections, where an internal ridge is commonly present.

Experimental data with direct relevance to HVAC ducts are those that were collected in ducts with hydraulic diameters greater than or equal to $15 \mathrm{~cm}$ without deliberately added roughness elements. All such data are presented in Figure 8. The deposition surface in all of these experiments was the floor of a horizontal duct, except in the case of Montgomery \& Corn (1970) where the entire internal perimeter of a round horizontal duct served as the deposition surface. The data collected by Kvasnak et al. (1993) for irregularly shaped particles flowing through a $15 \mathrm{~cm}$ wide and $2.5 \mathrm{~cm}$ high duct are 
included even though the hydraulic diameter of this duct is somewhat smaller than the others.

The data in Figure 8 are not expected to follow the same deposition regimes illustrated in Figure 2 because of the influence of gravity when deposition is occurring on a horizontal surface. The data appear to be quite scattered; however, much of the variation arises from the scaling by the friction velocity to yield the dimensionless quantities that are plotted. Deposition to the floor surface is controlled by gravity in these large ducts; therefore, with the diffusion-impaction and inertia-moderated regimes, particles deposit at approximately their gravitational settling velocity, regardless of the turbulence intensity. When scaled by the friction velocity, equal deposition velocities have different values of $V_{d}^{+}$for different turbulence intensities.

The only work in the literature to investigate deposition in a large duct to a surface other than the floor is from Sehmel (1973), who also measured deposition rates to the duct ceiling. No data are presented in the literature for deposition to the sidewall of a large duct, although Muyshondt et al. (1996) report a few data points for $20 \mu \mathrm{m}$ particles depositing to the walls of a $10.2 \mathrm{~cm}$ diameter vertical pipe. The data for deposition to the floor and ceiling of a $61 \mathrm{~cm}$ square duct as measured by Sehmel (1973) are shown in Figure 9. The measured deposition velocities to the ceiling are observed to be 1-2 orders of magnitude lower than those to the floors. This figure also more clearly shows the variation in dimensionless deposition velocities to floor surfaces with friction velocities described in reference to Figure 8.

The investigations of Adam et al. (1996) and Cheong (1997) are both directly concerned with particle deposition in HVAC ducts, but are of questionable value owing to the poor quality of the experimental methods used and the unclear reporting of data. Adam et al. measured particle penetration through a horizontal square duct of $30 \mathrm{~cm}$ width by monitoring upstream and downstream locations with infrared particle monitors. In addition to studying a straight duct, particle penetration through a flow reducer, a single $90^{\circ}$ duct bend, a double $90^{\circ}$ bend and a flow damper positioned at four different angles were measured. A polydisperse test aerosol was used and most data were presented as least-squares fits of the percentage of particles penetrating the duct versus the flow rate. The data of Adam et al. for the straight duct section is presented in the traditional method of $\tau^{+}$versus $V_{d}^{+}$in Figure 10. A particle diameter of $1.5 \mu \mathrm{m}$ was used to calculate representative values of $\tau^{+}$for the 0.5-2.0 $\mu \mathrm{m}$ polydisperse aerosol. A comparison with 
Figures 8 and 9 shows that these data are clearly inconsistent with almost all other data for deposition to nominally smooth surfaces. Cheong used experimental techniques and data reporting similar to Adam et al. when investigating the effect of the aspect ratio in rectangular ducts on deposition of a polydisperse aerosol of unreported size distribution. The data from Cheong for deposition in a $30 \mathrm{~cm}$ square duct are also shown in Figure 10 where, again, an average particle diameter of $1.5 \mu \mathrm{m}$ was assumed here to enable presentation of the data in this form. These data also seem inconsistent with previously observed trends and the inconsistencies are likely to be a consequence of the unreliable methods used in these measurements. Other than Adam et al., no data on particle deposition to separate ventilation duct components are reported in the literature.

Most experimental determinations of particle deposition from turbulent flow have been limited to deposition from fully developed flow in straight ducts, but a fully developed flow profile is not the norm in ventilation ducts. Deposition at bends and in duct branches from turbulent flow has rarely been reported in the literature, and there are no reports of such measurements in large-diameter ducts. Differences in particle deposition from developing flow and flow with a fully developed velocity profile have been mentioned by some researchers, but have not been the subject of systematic investigation. In most cases, deposition from the developing flow downstream of a duct inlet has been observed to be greater than that in fully developed flow (Chamberlain, 1967; Wells \& Chamberlain, 1967; Sehmel, 1968; Ilori, 1971). Occasionally the opposite trend has been observed (Friedlander \& Johnstone, 1957; Liu \& Agarwal, 1974). The effect has not often been reported quantitatively.

The overall data set in the literature for particle deposition from turbulent flow has addressed a broad range of experimental conditions. While results are often not directly comparable, and measured particle deposition rates, even within individual data sets, are frequently widely scattered, clear trends and broad consistency in the data can be observed. Direct relevance of the data set to ventilation ducts is limited. Of all data of reliable quality, only Sehmel (1973) and Lai (1997) have performed experiments in ducts similar to those found in HVAC systems. Only Sehmel (1973) has reported differences in particle deposition to the distinct surfaces in horizontal ducts. Air traveling through an HVAC system typically traverses several bends and branches that alter flow conditions from the fully developed state. No investigations have been reported regarding deposition at these sites from ducts with sizes relevant to building ventilation systems. There are no quantitative data on particle deposition from incompletely developed flows 
associated with inlet sections or flow after bends in large ducts. Complexities of air flow through HVAC ducts and the surface character of insulated or used and soiled ducts are two primary factors that set the real situation of particle deposition in ventilation ducts apart from all previous investigations. 


\section{Turbulent Airflow in Ventilation Ducts}

Most models of particle deposition from turbulent flows have focused on particle interactions with turbulent eddies in the near-wall region of the flow. The description of near-wall turbulence in this section is meant to provide a basis for better understanding the assumptions and limitations of the particle deposition models discussed in Section 4. The near-wall turbulence phenomena described are the fluctuating wall-normal velocity component, the eddy viscosity and organized vortices, bursts and down sweeps. Some key experiments and numerical investigations regarding these phenomena are discussed for turbulent flows near both smooth walls (Section 3.2) and rough walls (Section 3.2). A brief discussion of secondary flows in rectangular ducts and in duct bends is provided in Section 3.3.

\subsection{Description of Turbulent Flow near Smooth Walls}

For particles in the diffusion and diffusion-impaction regimes, deposition is dominated by particle behavior in a very thin region near the wall. An understanding of the structure of turbulent duct flow very near the duct wall illuminates particle deposition behavior observed both in experiments and in Lagrangian simulations. Very close to boundaries, variables are expected to be independent of flow Reynolds number when nondimensionalized by the near-wall scaling variables, the friction velocity $\left(u^{*}\right)$ and the kinematic viscosity $(v)$. Dimensionless quantities, sometimes called wall variables and signified by the + superscript, are formed by multiplying velocities by $1 / u^{*}$, lengths by $u^{*} / v$ and times by $u^{* 2} / v$.

\subsubsection{Fluctuating velocity component normal to a smooth wall}

Fluid velocities in turbulent flows are unsteady and appear to fluctuate randomly. Instantaneous velocity components in a turbulent flow can be expressed as a sum of the mean velocity component and a fluctuating velocity component as follows

$$
\begin{aligned}
& u=\bar{u}+u^{\prime} \\
& v=\bar{v}+v^{\prime} \\
& w=\bar{w}+w^{\prime}
\end{aligned}
$$

where $\bar{u}, \bar{v}$ and $\bar{w}$ are the time-averaged mean velocity components in the $x, y$ and $z$ (streamwise, wall-normal and spanwise for ducts) directions defined in Figure 11 and $u^{\prime}$, 
$v^{\prime}$ and $w^{\prime}$ are the fluctuating velocity components in the corresponding directions. These fluctuating velocity components play a significant role in the transport of heat, mass and momentum in turbulent flows. In particular, interactions between particles and the fluctuating air velocity component normal to the wall, $v^{\prime}$ in equation (21), were suspected to control particle deposition on surfaces in the earliest theories. A gradient in the wall-normal fluctuating air velocity component leads to a turbophoretic drift of particles toward a wall, as described later. The quantification of the wall-normal fluctuating velocity component was a point of disagreement amongst early particle deposition theories.

Investigations into near-wall turbulence have been frequently conducted in four distinct types of flows: turbulent boundary layers over a flat plate, channel flow, duct flow and pipe flow. Channel flow is flow between two infinite parallel plates and can be approximated experimentally in a duct with an aspect ratio greater than 5:1. Duct flow refers to flow in a duct of rectangular cross-section that, for this discussion, has an aspect ratio (width:height) of less than 5:1. Pipe flow is defined as flow through a tube of circular cross section. While each of these flows is distinct and may differ from each other far from the boundaries, the near-wall turbulence is expected to be similar in each case because the flow in this region is dominated by the presence of a single proximate wall in the near vicinity of the flow. Flow in HVAC ducts can usually be classified as duct flow or pipe flow.

Techniques for experimental measurement of turbulent fluctuating velocity components include hot wire anemometry (HWA), laser Doppler anemometry/velocimetry (LDA/LDV), and particle image velocimetry (PIV). The different methods yield similar results, and all have the limitation that velocities very close to a wall are difficult to measure and are less accurate than velocities measured far from the wall as a consequence of wall interferences. Laufer (1954) was one of the first to report experimental data regarding the near-wall normal fluctuating velocity component, $v^{\prime}$. This investigation considered airflow through a $25.4 \mathrm{~cm}$ diameter pipe at Reynolds numbers, based on the average flow rate and duct diameter, of 50,000 and 500,000. Figure 12 shows the data collected by Laufer plotted as the dimensionless root-meansquare (rms) wall-normal velocity, $v_{r m s}^{\prime+}$, versus the dimensionless distance from the wall, $y^{+}$, where $y$ is the distance from the wall and 


$$
\begin{aligned}
& v_{r m s}^{\prime+}=\frac{\sqrt{\overline{\left(v^{\prime}\right)^{2}}}}{u^{*}} \\
& y^{+}=\frac{y u^{*}}{v}
\end{aligned}
$$

Included in Figure 12 are the data collected by Durst et al. (1995) by LDA in oil flow through a $5 \mathrm{~cm}$ diameter pipe at Reynolds numbers from 7440 to 20,800. Durst et al. were able to make high quality measurements very close to the pipe wall by taking great care to minimize wall effects. The two data sets agree well over a range of flow Reynolds numbers due to the scaling of the fluctuating velocity components and the distance from the wall by the friction velocity and air viscosity. The values of $v_{r m s}^{\prime+}$ measured by Laufer are generally higher than those of Durst et al., probably because of larger wall interferences in the former case.

In recent years, much has been learned about turbulent flows by conducting numerical simulations on computers. Direct numerical simulation (DNS) provides the most accurate means of simulating turbulence. In DNS, the Navier-Stokes equations are solved numerically with resolution in time and space fine enough to capture even the smallest turbulent eddies. DNS is computationally intensive, and the need for computational power increases dramatically as the Reynolds number increases. Thus, DNS is limited to relatively low Reynolds numbers and short simulation times. Large eddy simulation (LES) is a method of generating a turbulent flow field that is not as severely restricted to low Reynolds number as DNS. In LES, only the large scale turbulent eddies are simulated directly, and the small scale eddies are modeled by a subgrid scale model. Information about the small-scale eddies is lost, but reasonably accurate modeling of the subgrid eddies is possible because the behavior of these eddies is more universal. These computational methods have proven to be very reliable and have the advantage over physical experiments of providing information on flows very close to boundaries.

Profiles of $v_{r m s}^{\prime+}$ versus $y^{+}$in channel flow at two different Reynolds numbers as calculated from the DNS of Moser et al. (1999) are shown in Figure 12 along with experimental pipe flow data. Equation 90 is discussed later, in section 4.4.1. The bottom panel of this figure shows the same data as the top panel with the axes scaled to focus attention on data near the wall. The profiles from the DNS at the two different Reynolds numbers diverge away from the wall, but converge for values of $y^{+}$less than 20 . 
Reasonable agreement between the DNS data and the physical experiments is observed. However, values of $v_{r m s}^{\prime+}$ immediately adjacent to the wall are lower in DNS than in experiments, possibly owing to wall interference in the physical experiments. The profiles calculated by DNS follow the quadratic relationship expected from theory (Chapman \& Kuhn, 1986).

DNS has been performed in square ducts (Gavrilakis, 1992; Huser \& Biringen, 1993). Profiles of $v_{r m s}^{\prime+}$ versus $y^{+}$near the center of the duct walls compare well to the results from channel DNS. In the DNS of square duct flows, values of ${v_{r m s}^{\prime+}}^{+}$were suppressed near the corners of square ducts relative to those near the center of the duct wall.

\subsubsection{Eddy viscosity}

The similarity between the mechanisms of mass and momentum transport in fluid flows gives rise to the analogy between the eddy viscosity inducing momentum transport and the eddy diffusivity inducing mass transport. In most theories of particle deposition from turbulent flow, the eddy diffusivity of particles has been assumed to be equal to the eddy diffusivity of the air, and specification of this parameter and its dependence on $y^{+}$has been a primary concern. The assumption of the equivalence of the eddy diffusivity to the eddy viscosity is valid for particles in homogeneous isotropic turbulence and for very small particles (i.e., those in the diffusion regime) near a wall. However, the assumption breaks down for larger particles, which possess significant inertia, especially in the vicinity of a wall where the turbulence is anisotropic.

It can be shown that substitution of equations (20)-(22) into the fluid momentum conservation equation and subsequent time averaging can lead to an expression for the fluid shear stress (Kay \& Nedderman, 1990):

$$
\tau_{a}=\rho_{a}\left(v \frac{\partial \bar{u}}{\partial y}-\overline{u^{\prime} v^{\prime}}\right)
$$

where $\tau_{a}$ is the time-averaged local shear stress in the air, $\partial \bar{u} / \partial y$ is the mean velocity gradient normal to the duct wall and $\overline{u^{\prime} v^{\prime}}$ is the time average of the product of the streamwise $\left(u^{\prime}\right)$ and wall-normal $\left(v^{\prime}\right)$ fluctuating velocity components. The first term on the right side of (25) is the contribution to shear owing to viscous forces and the second term is the shear stress induced by turbulent fluctuations. 
In analogy with the viscous transport of momentum by gradient diffusion through random interactions of molecules, turbulent momentum transport is often modeled as gradient diffusion by random interactions of turbulent eddies. The term representing the turbulent transport of momentum, $-\overline{u^{\prime} v^{\prime}}$, is often modeled by this expression:

$$
-\overline{u^{\prime} v^{\prime}}=\xi_{a} \frac{\partial \bar{u}}{\partial y}
$$

where $\xi_{a}$ is the eddy viscosity of the turbulent airflow. This eddy viscosity is not an intrinsic property of the air, but a property of the turbulent flow. Also, contrary to molecular viscosity, the value of $\xi_{a}$ is not constant, but varies strongly with distance from a wall, because the scale of the largest eddies increases with distance from a flow boundary. Combining (25) and (26), the shear stress per unit density of fluid may be expressed as

$$
\frac{\tau_{a}}{\rho_{a}}=\left(v+\xi_{a}\right) \frac{\partial \bar{u}}{\partial y}
$$

In this model, molecular viscosity and turbulent viscosity are assumed to additively and independently contribute to momentum transport.

Several correlations from the literature for the variation of eddy viscosity with distance from the wall are reproduced in Table 9. Based on continuity arguments, it is now well accepted that $\xi_{a}$ is proportional to $\left(y^{+}\right)^{3}$ in the close vicinity of a wall (Chapman \& Kuhn, 1986). The eddy viscosity correlations of Lin et al. (1953), Davies (1966a) (with Re = $10^{4}$ ) and Lai \& Nazaroff (2000) are plotted in Figure 13 and general agreement among the expressions is observed.

Figure 13 also clearly illustrates the very large change in eddy viscosity with distance from the wall. This large variation in eddy viscosity gives rise to three distinct regions of the turbulent flow: the viscous sublayer where momentum transport is dominated by the viscosity of air, the turbulent core where momentum transport is dominated by eddy viscosity, and the intermediate buffer layer where both molecular and eddy viscosities contribute significantly to momentum transport. These regions have historically been defined by

$$
\text { viscous sublayer: } \quad y^{+} \leq 5 \quad \xi_{a}<<v
$$




$\begin{array}{lll}\text { buffer layer: } & 5 \leq y^{+} \leq 30 & \xi_{a} \approx \nu \\ \text { turbulent core: } & y^{+} \geq 30 & \xi_{a}>>\end{array}$

The concept of eddy viscosity has proven useful for explaining momentum and gaseous mass transport phenomena in turbulent flows. The analogous concept of particle eddy diffusivity has achieved only limited success in explaining particle behavior in turbulent flows as will be discussed in more detail in Sections 4.1.7, 4.4.1 and 4.4.3.

\subsubsection{Organized structures in turbulence near a smooth wall}

Early studies of turbulence were framed around an assumption that turbulent motions were completely random and without structure. The flow visualization experiments of Kline et al. (1967) and Corino \& Brodkey (1969) were among the first to indicate a regular structure in the turbulent near-wall region, dominated by coherent vortical structures and intermittent down sweeping and bursting phenomena. It is now well recognized that these coherent structures are responsible for generating most of the turbulent fluctuation energy and for transporting most of the momentum associated with turbulence. There is also strong evidence from Lagrangian simulations that these structures contribute profoundly to particle deposition from turbulent flows. Because of their potential importance in influencing particle deposition, a brief description is merited of the form of the coherent near-wall turbulent structures. While ordered structures have been observed in near-wall turbulence, the region may still be rightfully characterized as having a high degree of disorder.

Cantwell (1981) provides a review of the early visualization and correlation studies and presents a detailed description of the near-wall turbulence structure and the associated bursting and down sweeping phenomena as it was understood at the time. Subsequent experimental studies and DNS investigations have partially modified this earlier understanding, but a consensus on the shape, strength, spatial orientation and dynamic role of most of the observed near-wall structures has yet to be reached. The presence of short-lived alternating streaks of high axial speed and low axial speed fluid immediately adjacent to the wall resulting from associated longitudinal streamwise vortices is well accepted. Observed dimensions of low speed streaks have varied widely, but central estimates of nondimensional lengths in the streamwise, wall normal, and spanwise directions of $\lambda_{x}{ }^{+} \sim 1000, \lambda_{y}{ }^{+} \sim 30$, and $\lambda_{z}{ }^{+} \sim 50$, respectively, are well accepted. An approximation of the instantaneous alternating arrangement of high speed and low speed 
streaks is shown in Figure 14. Individual streaks are temporal in nature, but the overall streaky structure is maintained because new streaks are formed as others subside. Low speed streaks have been observed to terminate with a 'burst' of the fluid away from the wall and into the turbulent core as suggested by Figure 15. Bursts of low speed fluid away from the wall have been associated with a corresponding downward sweep towards the wall of relatively high velocity fluid to fill the void left by the bursting fluid, and the frequency and intensity of these bursts and down sweeps were found to increase with increasing Reynolds number. The duration of burst and sweep events has been estimated to be approximately $20-25 \%$ of the mean time period between bursts (Chapman \& Kuhn, 1986).

Figure 15 also illustrates the association of low speed streaks in the viscous sublayer with pairs of coherent counter-rotating streamwise vortices in the buffer region as observed in early visualization studies. The streamwise vortices were observed to have similar dimensions as low speed streaks and to be the primary producers of turbulent shear stress. It is these eddies that have been implicated in Lagrangian simulations in playing an integral role in depositing particles in the diffusion-impaction regime to surfaces (Zhang \& Ahmadi, 2000).

Moin \& Mahesh (1998) chronicle the advances made by DNS to the understanding of the near-wall turbulence structure. The existence and extent of alternating streaks of highspeed and low-speed fluid and associated vortices have been confirmed by DNS. However, DNS data suggest that near-wall vortices are usually not paired with a counterrotating vortex, but commonly exist independently. DNS also suggests that the length of near-wall vortices is somewhat less than the length of the low speed fluid streaks adjacent to the wall and that experimentally observed bursts of fluid that were thought to terminate low speed streaks may be less crucial than originally thought.

While the current understanding of near-wall turbulence is far from complete, the basic structures of streaks and vortices near walls have been repeatedly observed in experiments and simulations. Interactions between these structures and particles may strongly influence particle deposition. The discovery of these near-wall structures provided the starting point for sublayer theories of particle deposition to walls from turbulent flows (Fichman et al., 1988; Fan \& Ahmadi, 1993). 


\subsection{Description of Turbulent Flow near Rough Walls}

Investigations into the near-wall turbulence structure in flows past rough walls are far more limited than smooth-walled studies. Roughness elements on walls can increase flow resistance compared to smooth walls because of form drag on the elements. In a similar way, roughness elements can enhance particle deposition by offering sites for particle impaction and by reducing the thickness of the viscous sublayer near the wall. The extent to which particle deposition may be enhanced is likely to depend on the size, shape and spacing of the roughness elements. Several key differences have been experimentally observed between the near-wall turbulence structure of smooth and rough walls. Thus, it is conceivable that surface roughness may also influence particle deposition by altering the turbulence structures that are expected to be responsible for particle deposition.

\subsubsection{Turbulence in rough wall boundary layers: Experiments}

Certain features of turbulence over rough walls have been observed to be different than features of turbulence near smooth walls. Grass (1971) studied the impact of sand-graintype roughness on turbulent flow characteristics by performing visualization experiments in an open water-channel flow with hydraulically smooth, transitionally rough $\left(k^{+}=20.7\right)$ and fully rough $\left(k^{+}=84.7\right)$ boundaries. This study confirmed the existence of low-speed streaks, sweeps and bursts in flows over rough walls, but noted that streamwise vortices were less apparent in rough-wall flows compared to smooth-wall flows. The spanwise extent of low-speed streaks was observed to be the same in smooth- and rough-wall cases. Notable differences between the smooth- and rough-wall cases were the location of the origin of the mean velocity profile and the difference in the profile of $v_{r m s}^{\prime+}$ versus

$y^{+}$. The mean velocity profile in the cases with rough surfaces was offset from the smooth-surface case to a location between the flat surface and the mean roughness height of the sand grains. The measured profile of $v_{r m s}^{\prime+}$ versus $y^{+}$agreed with the data of Laufer (1954) for the smooth wall; however, measured values of $v_{r m s}^{\prime+}$ near the wall were larger in the case of rough walls, and the measured increase was greater for larger roughness elements.

Krogstad et al. (1992) used HWA to measure mean and fluctuating velocities in a turbulent air boundary layer over a completely rough surface roughened by means of a wire mesh with a thickness of $1.55 \mathrm{~mm}$. They made comparisons to measurements over a 
smooth surface. Similar to the observations of Grass (1971), an offset in the mean velocity profile was noted as was a nearly twofold increase in $v_{r m s}^{\prime+}$ near the meshroughened surface as compared to the smooth boundary. Only small differences for profiles of $-\overline{u^{\prime} v^{\prime}}$ between smooth and rough walls were observed, suggesting that the eddy viscosity does not vary appreciably with changes in surface condition. Bursts and downsweeps observed over the mesh-roughened surface, and both phenomena occurred with a greater frequency and intensity than in the smooth-wall boundary layer. A key contribution of this work was the recognition that the impact of a rough wall on a flow may be felt not only in the immediate vicinity of the wall, but also well outside the buffer layer.

Grass et al. (1993) were the first to experimentally verify the existence of coherent streamwise vortical structures in transitional and fully rough turbulent flows. Visualization experiments were performed in an open channel water flow with the surface roughened by closely packed glass beads with diameters in the range 1.15-12 $\mathrm{mm}$. In contrast to the relatively constant dimensions observed in the smooth-wall case, the spanwise extent of the vortical structures was seen to be proportional to the size of the roughness elements.

The work of Krogstad \& Antonia (1999) highlighted the deficiencies of characterizing surface roughness by only the mean height. In this study, HWA measurements were made in a turbulent air boundary layer over repeated rib roughness and wire mesh roughness. Both cases were in the fully rough regime with an equivalent dimensionless sand grain roughness of 340 . For these two flows with identical mean velocity profiles, the profiles of $v_{r m s}^{\prime+}$ versus $y^{+}$differed dramatically near the wall with $v_{r m s}^{\prime+}$ values over the mesh being much greater than those over the ribs. Wall-normal velocity profiles were larger than smooth-wall profiles for both roughness cases. Labraga et al. (1997) also demonstrated the need to characterize roughness by more than just the mean height when investigating turbulent flows. These investigators made HWA measurements in a fully rough air channel roughened by elements of different aspect ratio and the frequency of bursts and sweeps in the flow were found to depend on the aspect ratio of the roughness elements. 


\subsubsection{Turbulence in rough walled channels and pipes: Experiments}

Surface roughness has been observed to have the opposite effect on profiles of $v_{r m s}^{\prime^{+}}$ versus $y^{+}$in the bulk flow of channel and pipe flow as compared with a turbulent boundary layer adjacent to a single bounding surface. Mazouz et al. (1998) present HWA measurements in an air channel flow with smooth and completely rough $\left(k^{+}=335\right)$ walls that were roughened by repeated ribs. Measurements suggested that values of $v_{r m s}^{\prime+}$ were suppressed in the case of fully rough walls compared to smooth walls throughout the entire channel. Measurements very close to the wall were not presented. These measured profiles compare favorably to the measurements of Sabot et al. (1977) in smooth and rough pipe flows. The experiments by Mazouz et al. and Sabot et al. dealt solely with the bulk airflow and did not address near-wall turbulence. Consequently, it is not clear whether such differences between boundary layer flows and pipe and channel flows exist in the near-wall region. Using the same experimental facility as Mazouz et al., Demare et al. (1999) studied the impact of fully roughened walls on bursting and down sweeping frequency. They found that the bursting frequency decreased by about a factor of two in the fully rough-wall case when compared to the smooth-wall conditions. Differences in the structures of the near-wall layers of channel and pipe flows as compared to boundary layer flows were attributed to interchanges of vortices between the different wall layers, which cannot happen in a turbulent boundary layer with a single wall.

\subsubsection{Turbulence in rough walled channels and pipes: Simulations}

As compared to smooth-wall investigations, DNS has played a less dominant role in the understanding of turbulence near rough walls because of the difficulty and computational expense of applying the no-slip boundary condition at the roughened surface. Choi et al. (1993) successfully implemented DNS in a channel with walls roughened by longitudinal riblets in the transitionally rough regime $\left(k^{+}=20\right.$ and 40$)$, an arrangement that has been observed to reduce drag relative to a smooth wall for some configurations. Profiles of $v_{r m s}^{\prime+}$ versus $y^{+}$near the wall were found to depend on the position above the tip or valley of the riblet and interactions between the riblet peaks and streamwise vortices were hypothesized to determine whether the riblet configuration would increase or decrease the drag of the flow. A novel idea put forth in this work was the definition of the offset 
in the velocity profile based on the fluctuating streamwise velocity component, $u^{\prime}$, rather than on the mean velocity.

Miyake et al. (2000) present DNS results for channel flow with one smooth wall and the other wall roughened by conical roughness elements to give a sand-grain type roughness with $k^{+} \sim 25$. In this simulation, very little difference was observed between profiles of $v_{r m s}^{\prime+}$ versus $y^{+}$over the smooth wall and the transitionally rough wall.

Friedrich et al. (2001) point out that a boundary condition of partial wall permeability accurately models wall roughness in DNS. They present results of DNS in a smooth pipe as well as in pipes with five different wall permeabilities. Values of $v_{r m s}^{\prime+}$ near the wall (presented in Wagner \& Friedrich, 1998) were found to be much higher near the permeable 'rough' walls than near the smooth wall. This contradicts the measurements of Sabot et al. (1977) in pipe flow, but is in qualitative agreement with measurements in turbulent boundary layers (Krogstad \& Antonia, 1999).

In summary, roughness has been observed to have a dramatic, though sometimes uncertain, effect on the structure of near-wall turbulence. Surface roughness has been accounted for in some particle deposition theories from the standpoint of reducing the particle transport distance across the viscous sublayer. The effect of changes in the structure of the near-wall turbulence owing to surface roughness on particle deposition processes has not been explored theoretically. Changes in turbulence structure caused by changes in surface roughness have an unknown impact on the deposition of particles entrained in flows near roughened walls.

\subsection{Secondary Flows}

Turbulent flow in ducts of rectangular cross section is different than flow in pipes or channels because the average wall normal and spanwise velocities have nonzero values in duct flows. At the corners of a duct, the interaction of the vertical and horizontal boundary layers establishes corner vortices, as shown in Figure 16, with flow toward the duct corners along the corner bisectors and flow toward the center of the duct along wall bisectors. Up to eight large-scale vortices in the plane normal to the mean flow direction may comprise the secondary flow in a square duct, and more vortices may be present as the duct aspect ratio increases. The intensity of this secondary flow is approximately 2$3 \%$ of the bulk velocity (Kay \& Nedderman, 1990). 
Another secondary flow of importance in ventilation ducts is that established in a bend. An example of the secondary flow in the plane normal to the mean flow that may be established at the outlet of a bend is shown in Figure 17. Two large, counter rotating vortices are established with flow along the horizontal centerline of the duct towards the outside of the bend and flow returning to the inside of the bend along the floor and ceiling of the duct. Experimental evidence indicates that from one to three secondary vortices may be established in a bend and that the shape and intensity of the secondary flow resulting from a bend depends on the Dean number

$$
\mathrm{De}=\operatorname{Re}\left(\frac{D_{h}}{2 R_{\text {bend }}}\right)^{0.5}
$$

The magnitude of these types of secondary flows is usually less than $10 \%$ of the axial velocity (Boersma \& Nieuwstadt, 1996). Bends skew the mean velocity profile toward the outer duct wall. Also, the LES result of Boersma \& Nieuwstadt of turbulent flow in a pipe bend indicates that $v_{r m s}^{\prime+}$ achieves higher values near the inner wall and lower values near the outer wall when compared to a straight pipe. To the best of our knowledge, the influence of secondary flows on particle deposition has not been addressed theoretically in the literature.

\subsection{Turbulent airflow summary}

Most models of particle deposition from turbulent flows require some representation of the near-wall turbulence. Advances in understanding near-wall turbulence have supported advances in predictive models of particle deposition. The structure of fully developed turbulence near smooth walls has been well studied; profiles of $v_{r m s}^{\prime+}$ and $\xi_{a}$ are predictable and near-wall vortices, low axial speed streaks, bursts and down sweeps have been well characterized. Turbulence near rough walls has been studied less frequently, but studies have shown the structure of turbulence near rough walls to be different than near smooth walls and dependent on the size and shape of the roughness elements. When modeling particle deposition to rough surfaces, no effort has yet been made to account for changes in the structure of turbulence owing to roughness; only the offset in the velocity profile due to the roughness elements has been considered. Likewise, secondary flow structures in rectangular ducts and in bends have not yet been incorporated into deposition models. 


\section{Predicting Particle Deposition Rates}

Particle deposition models provide estimates of particle deposition rates given information about the particles, the airflow in which the particles are suspended and the airflow conduit. Particle deposition rates predicted by models are most commonly expressed in terms of the dimensionless deposition velocity, $V_{d}^{+}$. Common input information for most models includes the particle size and density, the air velocity, and the hydraulic diameter of the duct. Some models are also able to predict the influence on deposition rates of duct-surface roughness. Others are able to predict deposition rates in the presence of thermal gradients or electrical fields. The orientation of the deposition surface influences particle deposition, but only a small fraction of models predict deposition rates to both horizontal and vertical surfaces.

No models presented in the literature have attempted to account for all forces that may act on a particle. Frequently, models have focused on predicting deposition rates of particles in a single deposition regime (diffusion, diffusion-impaction or inertiamoderated) because of the presumed unique mechanisms for deposition in each regime. Model evaluation by comparison to experimental data has been limited. Owing to the wide scatter in experimental data, it is difficult to make fine distinctions among models based on such comparisons under most circumstances.

This section first discusses the mechanisms that can cause particles to move relative to an air stream in section 4.1. The differences among empirical equations, Eulerian models and Lagrangian simulations are considered in section 4.2. Following is a review of the four main methods for predicting particle deposition rates: empirical equations (section 4.3), Eulerian models (section 4.4), sublayer models (section 4.5) and Lagrangian simulations (section 4.6). Section 4.7 provides a brief description of empirical models for predicting particle penetration rates through bends under turbulent flow conditions. This discussion follows a mostly chronological development of published models within each method of prediction.

\subsection{Particle Transport Mechanisms}

Forces and mechanisms influencing particle motion that are potentially present in turbulent ventilation duct flow are discussed in this section. All of the mechanisms are applicable to turbulent flow, but only turbulent diffusion and turbophoresis are unique to 
turbulent flow. All equations developed in this section are strictly applicable for rigid, spherical particles only; however, they are commonly applied in environmental aerosol dynamics models even when these conditions are not strictly met.

\subsubsection{Brownian diffusion}

Brownian motion is always present as a result of the random interactions between particles and air molecules. The flux of particles owing to Brownian diffusion is calculated by applying Fick's law of diffusion, written here for flux in one dimension:

$$
J_{B}=-D_{B} \frac{\partial C}{\partial y}
$$

where $J_{B}$ is the Brownian diffusive particle flux in the $y$-direction, $\partial C / \partial y$ is the $y$ component of the gradient in particle concentration and $D_{B}$ is the particle Brownian diffusivity. The Brownian diffusivity of a particle in air can be calculated by the StokesEinstein relation, corrected for slip:

$$
D_{B}=\frac{C_{C} k_{B} T}{3 \pi d_{p} \mu}
$$

where $k_{B}=1.38 \times 10^{-23} \mathrm{~J} / \mathrm{K}$ is Boltzmann's constant and $T$ is the absolute temperature. A net flux of particles generated by Brownian diffusion only exists in the presence of a nonzero concentration gradient. Brownian diffusion can be the dominant transport mechanism of very small particles over very small distances, but is a weak transport mechanism for particles larger than about $0.1 \mu \mathrm{m}$.

\subsubsection{Drag force}

Whenever there is relative motion between a particle and the surrounding air, the particle experiences a drag force from the air that tends to reduce that relative motion. In the general case, the drag force on a particle is calculated by

$$
F_{d}=\frac{\pi d_{p}^{2} \rho_{a}\left|u_{a}-v_{p}\right|\left(u_{a}-v_{p}\right) C_{d}}{8 C_{c}}
$$


where $u_{a}$ is the local air velocity, $v_{p}$ is the particle velocity, $C_{d}$ is the drag coefficient and the sign of the force is determined by the difference in the air and particle velocities. The drag coefficient of a sphere can be calculated by the following equations:

$$
\begin{array}{ll}
C_{d}=\frac{24}{\operatorname{Re}_{p}} & \operatorname{Re}_{p} \leq 0.3 \\
C_{d}=\frac{24}{\operatorname{Re}_{p}}\left(1+0.15 \operatorname{Re}_{p}^{0.687}\right) & 0.3<\operatorname{Re}_{p}<800
\end{array}
$$

where $\mathrm{Re}_{\mathrm{p}}$ is the particle Reynolds number

$$
\operatorname{Re}_{p}=\frac{d_{p}\left|v_{p}-u_{a}\right|}{v}
$$

Equation (32) is the drag coefficient for particles obeying Stokes law, while (33) represents an empirical fit to experimental observations. The derivation of Stokes law assumes that the fluid flow far from the particle is uniform and that the particle is not accelerating relative to the fluid. These assumptions are frequently violated for particles in HVAC duct flow, but the errors introduced by these violations are likely to be small. For particles that do not obey Stokes law because $\operatorname{Re}_{\mathrm{p}}>0.3$, the particle relaxation time could be calculated by

$$
\tau_{p}=\frac{C_{c} \rho_{p} d_{p}^{2}}{18 \rho_{a} v\left(1+0.15 \operatorname{Re}_{p}^{0.687}\right)}
$$

instead of by equation (13), where, in this case, $\mathrm{Re}_{\mathrm{p}}$ is evaluated at the initial velocity

difference $\left|v_{p, o}-u_{a}\right|$. However, this expression assumes that $\operatorname{Re}_{\mathrm{p}}>0.3$ for the entire relaxation period. In reality, as a particle relaxes towards the fluid velocity, the particle Reynolds number decreases towards zero. Even if the Reynolds number has a high initial value, some fraction of the relaxation period will occur with the particle obeying Stokes law. In most of the literature and in the results presented in this review, equation (13) is used to relate relaxation time to particle size.

\subsubsection{Gravitational force}

Particles more dense than air settle owing to the effects of gravitational acceleration. Neglecting buoyancy (appropriate for $\rho_{a}<<\rho_{p}$ ) the net gravitational force on a particle is 


$$
F_{g}=\frac{\pi}{6} d_{p}^{3} \rho_{p} g
$$

where $g$ is the gravitational acceleration, which at the surface of Earth is approximately equal to $9.81 \mathrm{~m} / \mathrm{s}^{2}$. A balance of the drag force with the gravitational force on a particle leads to a simple expression for the particle gravitational settling velocity

$$
v_{g}=\tau_{p} g
$$

The importance of gravitational settling increases with particle size. It is generally an unimportant mechanism for particles smaller than $0.1 \mu \mathrm{m}$ in diameter.

\subsubsection{Shear-induced lift force}

A particle entrained in a shear flow field may experience a lift force perpendicular to the main flow direction. The magnitude of this shear-induced lift force for particles in a constant shear flow far from any walls was first calculated by Saffman $(1965,1968)$ to be

$$
F_{l}=\frac{1.62 \mu d_{p}^{2}(d u / d y)}{\sqrt{v|d u / d y|}}\left(u-v_{p x}\right)
$$

where $d u / d y$ is the air velocity gradient normal to the duct wall and $v_{p x}$ is the particle velocity in the axial direction. The direction of the lift force depends on the relative velocity between the particle and the air in the $x$-direction (streamwise), evaluated at the particle center. A particle in a velocity gradient near a wall (where $d u / d y$ is positive) with a streamwise velocity higher than the air velocity will experience a negative lift force, i.e., towards the wall. A particle that lags the air stream in the streamwise direction has a lift force away from the wall.

Equation (38) as derived by Saffman has the constraints that

$$
\frac{d_{p}^{2}|d u / d y|}{v}<1 \quad \text { and } \quad \operatorname{Re}_{p}<\frac{d_{p}^{2}|d u / d y|}{v}
$$

McLaughlin (1991) performed a theoretical analysis in which the second constraint was relaxed and found the magnitude of the lift force to be less than or equal to that expressed by (38). Subsequent analyses by McLaughlin (1993) and Cherukat \& McLaughlin (1994) modified Saffman's expression to account for the presence of a wall and the nearwall expressions suggested a lessening of the lift force magnitude as the wall is 
approached. Wang et al. (1997) used the term 'optimum lift force' for the lift force when modified to relax the Reynolds number constraints and to account for the presence of a wall and this convention is adopted in this report. The lift force arises due to particle inertia and is most important for large particles. Analyses from Lagrangian simulations suggest that the lift force is most important very close to the wall $\left(y^{+}<20\right)$, where the velocity gradient is largest and the differences between particle and fluid velocities are greatest.

\subsubsection{Thermophoresis}

If a temperature gradient exists in an air volume, a particle in that volume tends to migrate towards the cooler region. The motion is the result of gas molecules on the warm side striking the particle with a greater average momentum than those on the cooler side. For larger particles, the establishment of a temperature gradient within the particle alters the gas temperature field near the particle and complicates the analysis. This motion can be evaluated by balancing a drag force with the thermophoretic force, which acts in the direction of decreasing temperature. This expression for thermophoretic force is given by Talbot et al. (1980):

$$
F_{t h}=-\frac{3 \pi \mu^{2} d_{p} H}{\rho_{a} T} \frac{d T}{d y}
$$

where $d T / d y$ is the $y$-component of the temperature gradient and $H$ is the thermophoretic force coefficient

$$
H=\left(\frac{2.34}{1+3.42 \mathrm{Kn}}\right)\left(\frac{k_{a} / k_{p}+2.18 \mathrm{Kn}}{1+2 k_{a} / k_{p}+4.36 \mathrm{Kn}}\right)
$$

Here, $k_{a}$ and $k_{p}$ are the thermal conductivities of the air and the particle material, respectively. The thermophoretic velocity, obtained when the thermophoretic force is balanced by drag is

$$
v_{t h}=\frac{-C_{c} v H}{T} \frac{d T}{d y}
$$

The thermophoretic velocity in a given temperature gradient is at a maximum and nearly independent of particle size for particles smaller than $1 \mu \mathrm{m}$. For larger particles, the thermophoretic velocity decreases with increasing particle size, provided that $k_{a} / k_{p}<\sim$ 
0.2. Lower values of $k_{a} / k_{p}$ also lead to lower thermophoretic velocities for larger particles. Thermal gradients are common in HVAC ducts because the delivered air is often heated or cooled and ducts are often outside of the thermal envelope of buildings.

\subsubsection{Electrostatic drift}

A charged particle in an electric field experiences an electrostatic force. The Coulomb force on a particle due to the electric field is calculated by

$$
F_{C}=q E
$$

where $q$ is the charge on the particle and $E$ is the electric field strength. The particle charge is calculated from its excess or deficit of electrons

$$
q=n e_{o}
$$

where $n$ is the number of electrons of deviation (including sign) from the electrically neutral state and $e_{o}$ is the charge of a single electron, $-1.6 \times 10^{-19} \mathrm{C}$.

Li \& Ahmadi (1993c) present an equation that predicts the electrostatic force on a charged particle near a conducting surface as

$$
F_{e}=q E-\frac{q^{2}}{16 \pi \varepsilon_{o} y^{2}}+\frac{q E d_{p}^{3}}{16 y^{3}}-\frac{3 \pi \varepsilon_{o} d_{p}^{6} E}{128 y^{4}}
$$

where $\varepsilon_{o}$ is the permittivity of air, equal to $8.86 \times 10^{-12} \mathrm{C}^{2} \mathrm{~N}^{-1} \mathrm{~m}^{-2}$. The terms on the right side of equation (45) respectively account for the Coulomb force, image force, dielectric force and dipole-dipole force. Li \& Ahmadi's analysis suggested that the dielectric force and the dipole-dipole force are negligible and that the Coulomb force dominates when an electric field is present. Because of the use of electrically conducting materials, significant electric fields are not expected in HVAC ducts. In the absence of an electric field, the only component of the electrostatic force that can influence particle motion is the image force. The image force is always directed towards a wall and is only appreciable extremely close to a wall. It only occurs near a conducting surface. Charges accumulated on electrically insulating materials may give rise to electric fields and influence the motion and deposition of charged particles. 


\subsubsection{Turbulent diffusion}

In the same way that fluctuating turbulent velocity components contribute to momentum transport in turbulent flows, turbulent fluctuations contribute to the diffusive flux of particles. The instantaneous particle concentration in a turbulent flow can be expressed as the sum of an average and a fluctuating concentration, just as the instantaneous turbulent velocity components are expressed in equations (20)-(22):

$$
C=\bar{C}+C^{\prime}
$$

where $C$ is the instantaneous concentration, $\bar{C}$ is the time averaged concentration and $C^{\prime}$ is the fluctuating concentration. Substitution of (46) into the particle mass conservation equation for duct flow and Reynolds averaging leads to a total particle diffusive flux (averaged over turbulent fluctuations) in the direction normal to the wall of

$$
J_{d i f f}=-D_{B} \frac{d \bar{C}}{d y}-\overline{v^{\prime} C^{\prime}}
$$

where $J_{\text {diff }}$ is the total diffusive flux and $\overline{v^{\prime} C^{\prime}}$ is the contribution to the total diffusive flux from turbulent fluctuations. Continuing the analogy with turbulent momentum transport, the term $\overline{v^{\prime} C^{\prime}}$ is commonly modeled for homogeneous turbulence by

$$
\overline{v^{\prime} C^{\prime}}=\xi_{p} \frac{d \bar{C}}{d y}
$$

so that the total diffusive flux can be represented by

$$
J_{d i f f}=-\left(D_{B}+\xi_{p}\right) \frac{d \bar{C}}{d y}
$$

Here, $\xi_{p}$ is the eddy diffusivity of the particle, which is often assumed to be equal to the eddy viscosity of air, $\xi_{a}$. This assumption implies that there is no slip velocity between the particle and the air, which is untrue in many circumstances. However, the equality of $\xi_{p}$ to $\xi_{a}$ has been shown to be true for larger particles in homogeneous turbulence, where $\xi_{a}$ is constant (Hinze, 1975). As with Brownian diffusion, there is no net particle flux owing to turbulent diffusion in the absence of a concentration gradient. 


\subsubsection{Turbophoresis}

In turbulence that is inhomogeneous, the gradient in turbulent fluctuating velocity components gives rise to turbophoresis, a particle transport mechanism that is distinct from turbulent diffusion. Because turbulent velocity fluctuations decay to zero at surfaces, near-wall turbulence is highly inhomogeneous, with a gradient in turbulence intensity as a function of near-wall distance. The velocity of a particle with sufficient inertia can be decoupled from the local air velocity because of the lag in particle response, as measured by its relaxation time. Where there is a gradient in turbulence intensity, the likelihood that an inertial particle is thrown to a region of lower turbulence intensity near a wall is greater than the likelihood that it will make the return journey away from the wall. This asymmetry leads to a net migration of particles in turbulent flows down a gradient in turbulence intensity and towards walls. Caporaloni et al. (1975) were the first to recognize this phenomenon and they calculated the turbophoretic velocity to be

$$
v_{t}=-\tau_{p} \frac{d\left(\overline{v_{p y}^{\prime}}\right)^{2}}{d y}
$$

The same expression was derived somewhat more rigorously by Reeks (1983).

Caporaloni et al. provided an expression relating the particle rms wall-normal velocity to that of the fluid. Subsequent investigators (Guha, 1997; Young \& Leeming, 1997) have proposed similar expressions for this relationship. Combining equations (13), (31), (32) and (50), and assuming that the drag force balances turbophoresis, the net turbophoretic force applied to a particle can be expressed as

$$
F_{t}=\frac{\pi}{6} \rho_{p} d_{p}^{3} \frac{d\left(\overline{v_{p y}^{\prime}}\right)^{2}}{d y}
$$

Frequently, turbophoresis has not been explicitly recognized in the literature, even though it proves to be a dominant transport mechanism in turbulent flows for some inertial particles near walls. In contrast to turbulent diffusion, turbophoresis gives rise to a flux of particles even in the absence of a concentration gradient. 


\subsubsection{Combining transport mechanisms}

In Eulerian particle deposition models, particle transport mechanisms are usually assumed to be additive. This assumption appears valid for most practical purposes; however, there are cases when mechanisms do not act independently, but influence one another. An example is the effect of crossing trajectories identified by Yudine (1959) in which turbulent diffusion is reduced owing to the influence of gravitational settling. Small particles are able to follow fluid streamlines, but large particles influenced by gravitational settling may lose contact with eddies before the eddies decay. The heavy particles thus undergo a less intense turbulent diffusion owing to a lower velocity correlation with the surrounding fluid.

\subsubsection{Other transport mechanisms}

The previously discussed transport mechanisms have all been included in at least one form of particle deposition model. Other forces to transport particles exist but have been shown to be negligible for particle-air systems. The Basset history force, the Magnus force due to particle spin, the buoyancy force, the hydrodynamic force due to the pressure gradient in the flow and the Faxen correction for unsteady Stokes flow are all routinely ignored (Maxey \& Riley, 1983).

\subsection{Methods: Empiricism, Eulerian Models \& Lagrangian Simulations}

There are four basic approaches to predicting particle deposition rates in turbulent flows: empirical equations, Eulerian modeling, sublayer modeling and Lagrangian simulation. Empirical equations are simple best fits to collected experimental data, while Eulerian models and Lagrangian simulations are theoretically based approaches to modeling particle behavior. In the Lagrangian approach, the air is considered a continuous phase and the trajectory of a single particle through the airflow is predicted from the sum of all forces acting on the particle. By solving particle trajectories for particles from several different initial locations, airflow with particles can be simulated and information about particle deposition can be obtained. The Eulerian approach treats both the particles and the air as separate continuous phases. Individual trajectories of particles are not calculated, rather the overall behavior of an ensemble of particles is predicted through the 
introduction of the volume-averaged concentration and the solution of the conservation equations for the particle phase. Both Eulerian and Lagrangian methods require knowledge of the turbulent flow field and are suitable for solid or liquid particles depositing from airflow in a duct. Comparisons of Eulerian and Lagrangian methods are available in the literature (Durst et al., 1984; Gouesbet \& Berlemont, 1999). In general, Lagrangian simulations require less conceptual modeling and capture more of the fundamental physics involved in particle motion, but at a much greater computational cost. Sublayer models are also discussed in this section, these models use a Lagrangian scheme to locate a single limiting particle trajectory in the near-wall region upon which deposition velocity predictions are based. Sublayer models are not fully-Lagrangian simulations that find deposition rates from large numbers of calculated particle trajectories and are thus classified separately from Eulerian models and Lagrangian simulations.

Most models and simulation approaches share some common limitations. Most predict the deposition of rigid spherical particles to a vertical wall so that gravity is not a direct factor in determining deposition. Thermal gradients and electrical fields are usually ignored. All models address deposition from fully developed turbulent flow only. Particle concentrations are assumed to be low enough to ignore particle-particle interactions. (Such interactions are unlikely to be a concern when applied to the case of particles in HVAC ducts.) The presence of particles is also assumed not to affect the structure of air turbulence. The duct is assumed to be a perfect sink for particles so that once a particle contacts a wall, it does not bounce or detach and become reentrained. In the following sections, when models are applied to simulate experiments, the conditions in the model applications are set to be as close as possible to the physical conditions of the experiments.

\subsection{Empirical Equations}

Due to the historical lack of a physically satisfying and well performing model of particle deposition from turbulent flow, empirical equations have frequently been proposed as the best means to predict particle deposition. The equations are generally successful in representing experimental data because they are adjusted to fit these data, but these equations offer little in the way of understanding particle behavior or predicting particle deposition from flow arrangements different than those in the experiments to which the

empirical equations are fit. The most common form of the proposed empirical equations 
for deposition to vertical surfaces in the three deposition regimes are as follows (Papavergos \& Hedley, 1984):

$$
\begin{array}{ll}
\text { diffusion regime }\left(\tau^{+}<\sim 0.1\right): & V_{d}^{+}=k_{1} \mathrm{Sc}^{-2 / 3} \\
\text { diffusion-impaction regime }\left(\sim 0.1<\tau^{+}<\sim 10\right): & V_{d}^{+}=k_{2} \tau^{+^{2}} \\
\text { inertia-moderated regime }\left(\sim 10<\tau^{+}\right): & V_{d}^{+}=k_{3}
\end{array}
$$

where $k_{1}, k_{2}$ and $k_{3}$ are empirical constants and the particle Schmidt number, important in the diffusion regime, is defined as

$$
\mathrm{Sc}=\frac{v}{D_{B}}
$$

Published empirical equations for predicting particle deposition to smooth surfaces in the three different deposition regimes are presented in Sections 4.3.1-4.3.3. The application of these empirical equations to nonvertical surfaces is discussed in Section 4.3.4 and published empirical equations for deposition to rough surfaces are considered in Section 4.3.5.

\subsubsection{Empirical equations for smooth surfaces in the diffusion regime}

In the diffusion regime where particles are very small and Brownian motion is an important particle transport mechanism, particle deposition is similar to deposition of gaseous species because of the extremely small particle inertia. The film model of mass transfer of a diffusive species to a wall theorizes a turbulent core perfectly mixed by eddies and a thin laminar film near the wall where only molecular processes occur. A sharp boundary is assumed to divide the turbulent core and the laminar layer leading to the concentration profile of a diffusive species shown in Figure 18. Based on the analogy between momentum transfer and mass transfer, the film model predicts the dimensionless deposition velocity of a species to be

$$
V_{d}^{+}=\sqrt{\frac{f}{2}} \mathrm{Sc}^{-1}
$$

and substitution of the Blasius formula, equation (8), for $f$ yields

$$
V_{d}^{+}=0.1989 \mathrm{Re}^{-1 / 8} \mathrm{Sc}^{-1}
$$


The film model is only a rudimentary theory that neglects the contribution of turbulent diffusion in the near-wall region. A key limitation is the implicit assumption that the particle boundary layer thickness is independent of the particle diameter. Smaller particles diffuse faster and have thicker boundary layers than larger particles; thus the true dependence of deposition velocity on the Brownian diffusivity is less than the proportional dependence predicted by equations (56) and (57). A more rigorous consideration of the development of the particle concentration boundary layer and its dependence on Brownian diffusivity suggests that the Schmidt number exponent should be $-2 / 3$ instead of -1 . Colburn's j-factor approach, based on an analogy to these analyses predicts (Kay \& Nedderman, 1990)

$$
V_{d}^{+}=\sqrt{\frac{f}{2}} \mathrm{Sc}^{-2 / 3}
$$

or

$$
V_{d}^{+}=0.1989 \mathrm{Re}^{-1 / 8} \mathrm{Sc}^{-2 / 3}
$$

after substitution of equation (8). This result is in agreement with the relationship suggested by equation (52) and the $-2 / 3$ exponent can be considered to have strong theoretical, as well as empirical justification. It should be pointed out that equations (56)(59) were developed for smooth surfaces only, as the analogy between momentum and mass transfer only holds when there is no contribution to the overall drag from form drag on roughness elements.

The main difference between equations (52) and (59) is the Reynolds number dependence in equation (59), but this dependence is very weak. Values of $k_{1}$ for equation (52) calculated by equation (59) range from 0.073 when $\mathrm{Re}=10^{3}$ down to 0.035 when $\mathrm{Re}=$ $10^{6}$. Values of $k_{1}$ recommended by various authors are summarized in Table 10 and these values are near the range predicted by Colburn's j-factor analogy. In the cases of Cleaver $\&$ Yates (1975) and Wood (1981b), these constants are not the result of fits to experimental data, rather they come from theoretical analyses coupled with simplifying mathematical approximations. While equation (52) is often treated as a simple empirical expression, it is more correctly a mathematical approximation to a more rigorous theoretical analysis, supported by experimental evidence. 
Empirical equations slightly different in form from equation (52) have also been proposed. Shaw \& Hanratty (1977) found the best fit to turbulent diffusional deposition data collected in a liquid flow system to be

$$
V_{d}^{+}=0.0889 \mathrm{Sc}^{-0.704}
$$

with the difference between the -0.704 and $-2 / 3$ exponents being statistically significant. Shimada et al. (1993) correlated the results of several numerical calculations of diffusional deposition by the equation

$$
V_{d}^{+}=\frac{2.4 \times 10^{-4} \operatorname{Re}^{0.92} D_{B}^{0.67}}{D_{h} u^{*}}
$$

where all variables are in SI units (i.e. length in $\mathrm{m}$, time in s). The purpose of casting the expression in this form was to compare it with the analysis of Friedlander (1977) who derived a theoretical expression for diffusional deposition from pipe flow assuming the particle eddy diffusivity to be equal to Lin et al.'s (1953) expression for eddy viscosity near the wall. The expression from Friedlander was

$$
\frac{V_{d} D_{h}}{D_{B}}=0.042 \operatorname{ReSc}^{1 / 3} \sqrt{f}
$$

which was recast by Shimada et al. as

$$
V_{d}^{+}=\frac{3.0 \times 10^{-4} \mathrm{Re}^{0.88} D_{B}^{0.67}}{D_{h} u^{*}}
$$

However, for clarity in the context of this discussion, equation (63) can be more simply rewritten by substitution of equations (6), (8) and (10) giving

$$
V_{d}^{+}=0.061 \mathrm{Sc}^{-2 / 3}
$$

using a value of $1.55 \times 10^{-5} \mathrm{~m}^{2} \mathrm{~s}^{-1}$ for the kinematic viscosity of air. Thus, although they appear significantly different, equations (61) and (62) reduce to the same form as equation (52), in agreement with other expressions for diffusional deposition derived from mathematical approximations.

A comparison of equation (52) with $k_{1}=0.063$ to the empirical expressions of equations (60) and (61) is presented graphically in Figure 19. Experimental data collected by Shimada et al. in a very small (6 $\mathrm{mm}$ diameter) tube are included on the figure for 
comparison. To eliminate the confounding influence of Reynolds number (e.g., equation (59)), only data collected at a Reynolds number of approximately $10^{4}$ are included on the figure. Setting $k_{l}$ equal to 0.063 in equation (52) is the same as applying Colburn's jfactor analogy at a Reynolds number of $10^{4}$. From Figure 19, it is easy to see that there is little difference between the expressions, especially for $\tau^{+}<0.1$ where the equations are most applicable. In addition, these equations demonstrate good agreement with the collected experimental data. Because particles that are strongly influenced by Brownian motion are insignificantly affected by gravity, these equations for the diffusion regime may be applied equally to horizontal and vertical surfaces.

\subsubsection{Empirical equations for smooth surfaces in the diffusion-impaction regime}

Most relevant experiments have studied particle deposition to vertical walls. Proposed empirical equations to predict deposition velocities for particles in the diffusionimpaction regime to vertical surfaces have largely been of the form expressed by equation (53). These equations represent simple best fits to experimental data and a summary of values for $k_{2}$ reported in the literature is given in Table 11. All of the proposed constants agree to within a factor of 2 .

Papavergos \& Hedley (1984) also proposed correlations of the form of equation (53) for horizontal floor and ceiling surfaces based on the data collected by Sehmel (1973).

These equations are

$$
\begin{array}{ll}
\text { horizontal floor: } & V_{d}^{+}=2 \times 10^{-3} \tau^{+^{2}} \\
\text { horizontal ceiling: } & V_{d}^{+}=4 \times 10^{-5} \tau^{+^{2}}
\end{array}
$$

They suggest that deposition to a floor surface is enhanced by a factor of 3-6 compared to wall deposition whereas ceiling deposition is diminished by a factor of 9-15 compared to deposition to a wall.

Erhart (1986) found that a form of equation (53), modified by a factor proposed by Gieseke et al. (1980) to account for variations in Reynolds number, fit his data better than the unmodified equation. The modified equation is

$$
V_{d}^{+}=6.2 \times 10^{-4} \tau^{+^{2}}+2.0 \times 10^{-8} \operatorname{Re}
$$


The term with the Reynolds number represents an ad hoc modification that is unlikely to be useful over a wide range of Reynolds numbers.

Muyshondt et al. (1996) assumed the deposition velocity could be correlated to both $\tau^{+}$ and Re and fit the experimental data they collected in the diffusion-impaction regime to a sigmoid curve. The best fit was found to be

$$
\begin{aligned}
& V_{d}^{+}=a_{1} \exp \left[-0.5\left(\frac{\operatorname{Re}-a_{2}}{a_{3}}\right)^{2}\right]+a_{4} \exp \left[-0.5\left(\frac{\ln \left(\tau^{+}\right)-\ln \left(a_{5}\right)}{a_{6}}\right)^{2}\right] \\
& a_{1}=0.0226 \quad a_{4}=0.1394 \\
& a_{2}=4.03 \times 10^{4} \quad a_{5}=49.0 \\
& a_{3}=1.533 \times 10^{4} \quad a_{6}=1.136
\end{aligned}
$$

with the limitations that $0.1<\tau^{+}<100$ and $2500<\operatorname{Re}<50,000$. While it is generally true that increasing the number of fitting variables can improve the quality of a modelmeasurement comparison, it does so at the cost of losing the causal connections between the model equation and the underlying physical process. Thus, while the form of equations (52) and (53) reveal some mechanistic underpinnings, equation (68) reflects pure empiricism.

Dimensionless deposition velocities predicted by equation (53), with $k_{2}=4.5 \times 10^{-4}$, and by equations (67) and (68) are compared to the experimental data of Liu \& Agarwal (1974) in Figure 20. The data were collected for particles in the diffusion-impaction regime depositing to the walls of a $1.27 \mathrm{~cm}$ diameter tube at a nominal Reynolds number of 10,000. Equation (53), which is an empirical fit to the data, agrees well with the data in the diffusion-impaction regime as expected. Equations (67) and (68) both seem to overpredict deposition velocities for low values of $\tau^{+}$. This overprediction is likely the result of making the original empirical fit to data that were collected by techniques that were insufficiently sensitive to distinguish among low values of $V_{d}^{+}$in the range of $10^{-3}-$ $10^{-6}$.

\subsubsection{Empirical equations for smooth surfaces in the inertia-moderated regime}

For relatively large particles depositing to vertical surfaces, dimensionless deposition velocities have been observed to be relatively independent of particle size. The Reynolds analogy for mass transfer to pipe walls in a turbulent flow assumes that there is no 
viscous layer resistance to mass transfer near to the wall and that mass transfer rates are set by resistance in the turbulent core. This assumption is approximately true for large particles and the result of the Reynolds analogy for mass transfer is

$$
V_{d}^{+}=\sqrt{\frac{f}{2}}
$$

This result predicts deposition velocities to be independent of particle size and to be slightly dependent on the flow Reynolds number via the friction factor (equations (8) and (9)).

Several investigators have suggested that deposition velocities to vertical surfaces for particles in the inertia-moderated regime are constant and have recommended specific values for $k_{3}$ to be used in equation (54). These recommended values are given in Table 12 and there is little variation in the reported constants.

Several investigators have noted a decrease in experimentally observed deposition velocity as particle size increases through the inertia-moderated regime. Some have suggested that this phenomenon is best explained as the result of large particles bouncing upon impact with the wall or resuspending into the flow after deposition, but some have proposed that the reduction in deposition velocity is due to the decreased response to turbulent velocity fluctuations of the very large particles. Reeks \& Skyrme (1976) provide a theoretical expression to calculate particle deposition in the inertia-moderated regime that predicts decreased deposition with increased particle size. Wood (1981b) developed a simple mathematical approximation to this result and adjusted the constants to fit Liu \& Agarwal's (1974) data for large particle deposition. The result from Wood is

$$
\begin{array}{ll}
V_{d}^{+}=\frac{2.6}{\sqrt{\tau^{+}}}\left(1-\frac{50}{\tau^{+}}\right) & \tau^{+} \geq 270 \\
V_{d}^{+}=0.13 & 17 \leq \tau^{+}<270
\end{array}
$$

These equations predict a moderately decreasing deposition velocity with increasing particle relaxation time, for $\tau^{+} \geq 270$.

A comparison is made between equation (54) with $k_{3}=0.18$ recommended by Papavergos \& Hedley (1984), the Reynolds analogy of equation (69) and equation (70) from Wood (1981b) in Figure 21. The experimental data in the figure were collected by Liu \& 
Agarwal (1974) in a $1.27 \mathrm{~cm}$ tube at a nominal Reynolds number of 50,000. The equations recommended by Wood show excellent correlation with the data as expected because the equation was fit to these data. The constant deposition velocities suggested by other researchers and by the Reynolds analogy all show reasonable agreement (in magnitude if not in exact value) with the data.

\subsubsection{Synthesis of empirical equations}

Although most empirical equations are valid for only one deposition regime, deposition across the entire range of particle sizes is easily predicted by a simple combination of equations. Empirical equations in the form of equations (52)-(54) can be applied to vertical surfaces across all deposition regimes when configured in the following manner:

$$
\begin{array}{ll}
V_{d}^{+}=k_{1} \mathrm{Sc}^{-2 / 3}+k_{2} \tau^{+^{2}} & \text { if } k_{1} \mathrm{Sc}^{-2 / 3}+k_{2} \tau^{+^{2}} \leq k_{3} \\
V_{d}^{+}=k_{3} & \text { if } k_{1} \mathrm{Sc}^{-2 / 3}+k_{2} \tau^{+^{2}}>k_{3}
\end{array}
$$

To apply empirical equations of this type to horizontal surfaces, Kvasnak \& Ahmadi (1996) added a simple modification to account for the effect of gravitational settling on the particle deposition velocity. The result is

$$
V_{d}^{+}=k_{1} \mathrm{Sc}^{-2 / 3}+k_{2} \tau^{+^{2}}+g^{+} \tau^{+}
$$

where $g^{+}$is the dimensionless gravitational acceleration defined by

$$
g^{+}=\frac{g v}{u^{* 3}}
$$

and $g$ is positive for a floor and negative for a ceiling surface. In equation (73) the first term on the right side accounts for Brownian diffusion, the second term accounts for interactions between particle inertia and turbulent eddies and the final term accounts for gravitational settling. Equation (73) can be applied to horizontal surfaces for all particle sizes. The leveling of deposition velocities for particles in the inertia-moderated regime is not expected to be observed when considering deposition to ventilation duct floors. At friction velocities typically found in ducts, the transport of particles with dimensionless relaxation times larger than 10 is strongly influenced by gravity. As particle size increases in the inertia-moderated regime, deposition rates are expected to increase owing to increases in the gravitational settling rate. 
Equation (73), with representative values of $k_{1}=0.057$ and $k_{2}=4.5 \times 10^{-4}$, is compared to the data collected by Sehmel (1973) for particle deposition to the floor and ceiling of a horizontal square duct in Figure 22. Also included on this figure are equations (65) and (66), the empirical fits to the data of Sehmel (1973) for the floor and ceiling. Equation (73) compares favorably to the experimental data for the floor. Also, the slope of equation (73) follows the slope of the experimental data more closely than equation (65), implying that deposition in this case is dominated by gravity as expressed in (73) and not by inertial interactions with turbulence as expressed by (65). Equation (73) is less successful in this case at correlating the experimental data for deposition to the ceiling. The excellent agreement between equation (65) and the experimental data for the ceiling derives from the fact that (65) was developed as a correlation to these data.

Following the same technique of linear summation of deposition mechanisms as in equation (73), successes have been reported for estimating deposition velocities of particles under the influence of thermal gradients (He \& Ahmadi, 1998) and electrical fields (Fan \& Ahmadi, 1994) by rather simple equations.

\subsubsection{Empirical equations for rough surfaces}

All of the preceding empirical equations are recommended only for smooth walls. Wood (1981b) modified the theoretical results of Davies (1966b) for deposition of particles in the diffusion regime to account for surface roughness. Wood recommended the following mathematical approximations for deposition of particles in the diffusion regime and for wall roughness in the hydraulically smooth or transitionally rough regime

hydraulically smooth $\left(0.45 k^{+} \leq 5\right)$ :

$$
\begin{aligned}
& \phi_{k}=\frac{k^{+} \mathrm{Sc}^{1 / 3}}{32.2} \\
& V_{d}^{+}=\left[24.2+14.5 \mathrm{Sc}^{-2 / 3}\left(\frac{2 \pi}{3 \sqrt{3}}-\frac{k^{+} S c^{1 / 3}}{27.6}\right)\right]^{-1} \quad \text { if } \quad \phi_{k}<1 \\
& V_{d}^{+}=\left[\frac{1524}{0.45 k^{+}\left(0.9 k^{+}-5 / \phi_{k}\right)^{-6.3}}\right]^{-1} \quad \text { if } \phi_{k} \geq 1
\end{aligned}
$$

transitionally rough $\left(5 \leq 0.45 k^{+} \leq 70\right)$ : 


$$
V_{d}^{+}=\frac{0.2}{\ln \left(\frac{25.2}{0.45 k^{+}-4.8}\right)}
$$

A correlation for the deposition velocity of particles in the diffusion regime to completely rough surfaces based on a theoretical analysis is (Davies, 1983)

$$
V_{d}^{+}=0.080 \mathrm{Sc}^{-1 / 2}
$$

This equation has a form similar to equation (52), but the dependence on Sc differs.

Fan \& Ahmadi (1993) present a perturbation solution to the equations of particle motion used in their sublayer model to predict deposition rates of particles in the diffusionimpaction regime to vertical surfaces with small amounts of roughness. The resulting empirical equation is in a complex form with many variables that need to be calculated, but the calculations are straightforward. The empirical equation gave predictions similar to their sublayer model for both smooth and rough surfaces. Other than the perturbation solution of Fan \& Ahmadi (1993), We are unaware of any empirical expressions for predicting deposition of particles outside the diffusion regime to rough surfaces. We are also unaware of any data in the diffusion regime investigating deposition to rough surfaces with values of $k^{+}<140$, with which to compare equations (75)-(78).

\subsection{Eulerian Models}

There are three main classes of Eulerian models for predicting particle deposition rates: gradient diffusion models, free-flight models and turbophoretic models. Gradient diffusion and free-flight models entail solving the particle mass conservation equation. Turbophoretic models solve the particle mass and particle momentum conservation equations. Gradient diffusion models consider turbulent and Brownian diffusion to be the only mechanisms of particle transport that induce deposition. Free-flight models explain particle deposition as a combination of diffusion towards a wall followed by a final step to the wall in 'free-flight' at a velocity much higher than that of the air near the wall. Turbophoretic models consider particle transport by both diffusion and turbophoresis and offer a more physically satisfying explanation of particle deposition compared to free-flight models. Through the inclusion of the particle momentum equation, turbophoretic models are also more readily able to be adapted to include forces like shear-induced lift than are other Eulerian models. 
The development of free-flight models for smooth and rough surfaces are respectively discussed in Sections 4.4.1 and 4.4.2. Gradient diffusion models and turbophoretic models are reviewed in Sections 4.4.3 and 4.4.4, respectively.

\subsubsection{Free-flight models for smooth surfaces}

The first major theory of particle deposition from turbulent flow was the free-flight theory proposed by Friedlander \& Johnstone (1957). The theory aimed to explain the extremely rapid increase in deposition velocity to a vertical surface with small increases in particle size in the diffusion-impaction regime observed in their own experiments. Many subsequent authors have added modifications to this original theory and good reviews of the evolution of the free-flight theory are available (Papavergos \& Hedley, 1984; Wallin, 1994). This section first describes the general ideas of the free-flight theory then discusses the assumptions made by various investigators to implement the theory. All of these free-flight models predict deposition to vertical surfaces only.

The free-flight deposition theory is based on the idea that particles are able to deposit onto a wall by traversing the laminar region near the wall in free flight instead of diffusing through a near-wall concentration boundary layer. Particles entrained in turbulent eddies are assumed to travel towards the wall by a combination of turbulent and Brownian diffusion to the relatively quiescent region adjacent to the wall. At this point the turbulent eddies dissipate, but particles continue moving toward the wall in free flight. Particles impact on the surface where they deposit owing to their inertia. Particles are assumed to stop diffusing towards the wall and begin free flight to the wall at a distance equal to the particle stopping distance away from the wall. The stopping distance of a particle is the characteristic distance that the particle, given an initial velocity, will travel through stagnant air before coming to rest. The stopping distance is calculated by means of this expression:

$$
S=\tau_{p} v_{p, o}
$$

where $S$ is the stopping distance and $v_{p, o}$ is the initial velocity of the particle. The stopping distance can be made dimensionless by this expression:

$$
S^{+}=\frac{S u^{*}}{v}
$$


Mathematically, the free-flight theory is a solution of the particle mass conservation equation in the direction normal to a wall. Writing this equation for turbulent flow and subsequently applying Reynolds averaging leads to Fick's law of diffusion, modified to include the effects of turbulent diffusion:

$$
J_{\text {diff }}=\left(D_{B}+\xi_{p}\right) \frac{d \bar{C}}{d y}
$$

This is a restatement of equation (49), although here the negative sign on the right hand side is dropped and flux toward the wall is defined to be positive. Equation (82) can be written in dimensionless form as

$$
V_{d}^{+}=\left(\frac{D_{B}}{v}+\frac{\xi_{p}}{v}\right) \frac{d C^{+}}{d y^{+}}
$$

where

$$
C^{+}=\frac{\bar{C}}{C_{b u l k}}
$$

and $C_{b u l k}$ is the time-averaged particle concentration in the turbulent core of the duct, which is assumed to be constant.

The capture distance, $\Delta$, is defined as the distance from the wall at which the depositing particle begins its free flight to the wall. The particle is assumed to undergo Brownian and turbulent diffusion from the core region to the capture distance. Equation (83) is integrated from the turbulent core, where $C^{+}=1$, to the particle capture distance to yield

$$
\frac{1-C_{\Delta^{+}}^{+}}{V_{d}^{+}}=\int_{\Delta^{+}}^{y^{+}\left(C^{+}=1\right)} \frac{d y^{+}}{\left(\frac{D_{B}}{v}+\frac{\xi_{p}}{v}\right)}
$$

where $y^{+}\left(C^{+}=1\right)$ is the dimensionless distance from the duct wall to the turbulent core, $\Delta^{+}$is the dimensionless particle capture distance, and $C_{\Delta^{+}}^{+}$is the dimensionless particle concentration at the dimensionless capture distance. The parameter $C_{\Delta^{+}}^{+}$is an unknown quantity that is not easily measured. This variable may be eliminated from the analysis by substituting for the flux of particles at the capture distance, which is defined as follows: 


$$
J=\left(v_{p y} \cdot \bar{C}\right)_{\Delta^{+}}
$$

where $v_{p y}$ is the particle velocity normal to the wall. The dimensionless deposition velocity can be written

$$
V_{d}^{+}=\frac{J}{C_{b u l k} u^{*}}
$$

Substitution of equation (86) into (87) gives

$$
V_{d}^{+}=\left[\left(\frac{v_{p y}}{u^{*}}\right)\left(\frac{\bar{C}}{C_{b u l k}}\right)\right]_{\Delta^{+}}=v_{p y, \Delta^{+}}^{+} C_{\Delta^{+}}^{+}
$$

where $v_{p y, \Delta^{+}}^{+}$is the dimensionless $y$-component of particle velocity at $\Delta^{+}$. Equation (88) can then be substituted into (85) to eliminate $C_{\Delta^{+}}^{+}$and give a general form of the freeflight model:

$$
\frac{1}{V_{d}^{+}}=\frac{1}{v_{p y, \Delta^{+}}^{+}}+\int_{\Delta^{+}}^{y^{+}\left(C^{+}=1\right)} \frac{d y^{+}}{\left(\frac{D_{B}}{v}+\frac{\xi_{p}}{v}\right)}
$$

The dimensionless deposition velocity may be evaluated given information or appropriate assumptions about $\xi_{p}, \Delta^{+}$, and $v_{p y, \Delta^{+}}^{+}$. Not all free-flight models conform exactly to equation (89), but this form provides a useful framework for looking at the influence of different assumptions on the predictions of free-flight models. Often, investigators have focused on integrating equation (89) and expressing it in the form of an analytical equation to allow for easier evaluation of deposition velocities.

In their original model, Friedlander \& Johnstone (1957) ignored Brownian diffusion and assumed the particle eddy diffusivity, $\xi_{p}$, to be equal to Lin et al.'s (1953) correlations for the eddy viscosity, $\xi_{a}$. The dimensionless capture distance, $\Delta^{+}$, was assumed to be equal to the dimensionless stop distance of the particle, $S^{+}$. The initial dimensionless velocity of the particle before making the free flight to the wall, $v_{p y, \Delta^{+}}^{+}$, was assigned a constant value of 0.9 regardless of particle size. This value for ${v_{p y, \Delta^{+}}^{+}}^{p y, \Delta^{+}}$was chosen to match the wall-normal, root-mean-square fluctuating velocity of the air in the turbulent core as measured by Laufer (1954) (see Figure 12). Resistance to particle transport through the turbulent core to a distance of $y^{+}=30$ was calculated by the Reynolds analogy. This resistance was added in series with the resistance represented by the integral in equation 
(89), which was integrated from $y^{+}=\Delta^{+}$to $y^{+}=30$ (with $D_{B}=0$ ) to determine $V_{d}^{+}$. The first term on the right side of equation (89) was ignored in this analysis. This is equivalent to assuming that the normalized particle concentration at $\Delta^{+}$is very much smaller than one. A summary of the assumptions made by Friedlander \& Johnstone and by several other investigators that have proposed variations on this free-flight model is given in Table 13. Friedlander \& Johnstone summed the resistances to particle transport in the turbulent core, buffer layer and viscous sublayer. The resistance in the turbulent core was derived from the Reynolds analogy and the resistance in the buffer layer and viscous sublayer was calculated by integration of equation (89). The result was three expressions for the dimensionless deposition velocity that depend only on the particle stopping distance; the proper equation to apply is determined by the magnitude of the dimensionless stopping distance. A comparison of the theory of Friedlander \& Johnstone with the data collected by Liu \& Agarwal (1974) is presented in Figure 23. The agreement between model and measurement is favorable, but the discontinuities in the model predictions are physically unrealistic.

Davies (1966b) suggested many modifications to the free-flight theory of Friedlander \& Johnstone (1957). Davies noted that the capture distance should include the particle radius to account for the interception effect. Also, he suggested that the initial free-flight velocity, $v_{p y, \Delta^{+}}^{+}$, be equated to the local root-mean-square of the wall-normal fluctuating air velocity component of the fluid instead of a constant value as recommended by Friedlander \& Johnstone. Davies approximated the measurements of Laufer (1954) for the rms wall-normal fluctuating velocity of the fluid by this expression:

$$
v_{r m s}^{\prime+}=\frac{y^{+}}{y^{+}+10}
$$

This profile is compared to data from experiments and DNS in Figure 12. Far from the wall, equation (90) approximately follows the trends in the data. Near the wall, in the bottom panel of the figure, equation (90) predicts values of $v_{r m s}^{\prime+}$ that are higher than the data. This profile does not predict the near-wall dependence of $v_{r m s}^{\prime+}$ on $y^{+2}$ that is expected from theory (Chapman \& Kuhn, 1986).

Davies also included the effects of Brownian diffusion in his analysis for cases when the dimensionless capture distance is less than $20\left(D_{B} / v\right)^{1 / 3}$. In addition, a continuous correlation for the eddy viscosity was utilized instead of the correlation proposed by Lin 
et al. (1953). The assumptions in this free-flight model are much more physically satisfying than those in Friedlander \& Johnstone's work because the initial free-flight velocity is not assigned arbitrarily, but is equal to the local fluid velocity fluctuations at the point at which free flight begins. Despite these apparent improvements in assumptions, the theory of Davies is observed to considerably underpredict the experimental data in Figure 23. The effect of Brownian diffusion in this model is readily apparent at low relaxation times where deposition velocity is predicted to increase as particle size decreases.

Liu \& Ilori (1974) sought to explain the discrepancy between the model of Davies (1966b) and the experimental data by suggesting that the particle eddy diffusivity was not equal to the fluid eddy viscosity as had previously been assumed. Liu \& Ilori proposed that the particle eddy diffusivity was greater than the eddy viscosity, especially for large particles, and argued that the particle eddy diffusivity should be calculated by

$$
\xi_{p}=\xi_{a}+v_{r m s}^{\prime 2} \tau_{p}
$$

In the work of Liu \& Ilori (1974), this equation for the particle eddy diffusivity, along with Davies' (1966b) expression for $v_{r m s}^{\prime+}$ (equation 90), and Owen's (1960) expression for the eddy viscosity of air, were substituted into a variant of equation (89). The limits of the integration were chosen to be the dimensionless duct centerline and the dimensionless particle capture distance, $\Delta^{+}$. Because the resulting integral expression was improper at the duct centerline, the equation was solved by analogy to the similar case of diffusion of a vapor from turbulent flow to a pipe wall. The model of Liu \& Ilori is compared to the relevant experimental data in Figure 23, and very good agreement with this data set is observed. However, the form of the expression for the particle eddy diffusivity given in (91) lacks a strong theoretical foundation. The eddy diffusivity of particles has been shown to be equal to the eddy viscosity of air over a long period of time and this modification really amounts to a clever semiempirical tuning of the freeflight model to better fit the data. Brownian diffusion was neglected in the development of this theory, as is evident by the monotonic rise in deposition velocity for $\tau^{+}<0.1$.

The free-flight theories of Davies (1966b) and Liu \& Ilori (1974) were significantly different in terms of basic assumptions from the original theory of Friedlander \& Johnstone (1957). Numerous other free-flight theories have been proposed that differ from one of these theories in only subtle ways. Owen (1960) proposed a free-flight 
theory of particle deposition independent of Friedlander \& Johnstone in which $\Delta^{+}$was assumed to be 1.6 regardless of particle size. This highly questionable assumption leads to a theory that predicts no variation in deposition with particle size, a prediction clearly at odds with experimental findings. The work of Owen, while being based on unrealistic assumptions, was the only free-flight model to address the issue of particle deposition to horizontal surfaces, such as floors and ceilings. Beal (1970) proposed a theory similar in most ways to Friedlander \& Johnstone's, but with an even higher initial free-flight velocity based on the local axial fluid velocity. Most subsequent investigators have found this proposed free-flight velocity to be unrealistically high. The theory of Beal introduced the concept of a particle sticking probability as a means of accounting for particle bounce, but only sticking probabilities equal to unity were used in his analysis. A free-flight theory incorporating a different correlation for the eddy viscosity of air was proposed by Wasan et al. (1973). Forney \& Spielman (1974) modified Friedlander \& Johnstone's theory to eliminate the discontinuities in the predicted relationship between deposition velocity and relaxation time.

In conclusion, although some free-flight theories give predictions of deposition that compare favorably to the best available experimental data, these theories are often based on questionable assumptions. The free-flight theory based on the most realistic assumptions, that of Davies (1966b), predicts deposition velocities 1-2 orders of magnitude lower than those observed experimentally in portions of the diffusionimpaction regime.

\subsubsection{Free-flight models for rough surfaces}

The first modeling work to attempt to account for the effect of deposition surface roughness was that of Browne (1974) who followed a suggestion made by Davies (1966a). Browne used all the same assumptions as Davies (1966b) except that the dimensionless capture distance was modified to account for surface roughness. The dimensionless capture distance was defined as

$$
\Delta^{+}=S^{+}+r_{p}^{+}+k^{+}+\sigma_{k}^{+}-e^{+}
$$

where $r_{p}{ }^{+}$is the dimensionless particle radius, $\sigma_{k}{ }^{+}$is the dimensionless standard deviation of the roughness height and $e^{+}$is the dimensionless offset in the axial air velocity profile owing to the roughness elements. These dimensionless parameters are made 
nondimensional by multiplying the dimensional parameters by the factor $u^{*} / v$. The dimensional forms of these parameters are defined graphically in Figure 24. The definition of the capture distance in this manner implies that particles deposit above the mean roughness height by a distance equal to one roughness height standard deviation. Browne recommends direct measurement of $k$ and $\sigma_{k}$. For cases in which the standard deviation in roughness height is not measurable, he suggests the following correlation:

$$
\sigma_{k}^{+}=0.17 k^{+}
$$

The point where the axial air velocity profile decays to zero is located somewhere between the peaks and the troughs of the roughness elements. The dimensionless height of this origin in the velocity profile relative to the lowest valleys in the roughness elements was estimated by Browne to be

$$
e^{+}=0.53 k^{+}+0.0034 k^{+2}
$$

This correlation is based on three measurements made by Grass (1971) in a water channel flow with sand-grain type roughness elements.

With the simple modification of the particle capture distance represented by equations (92)-(94), Browne modified the model of Davies (1966b) to account for the effect of surface roughness on particle deposition velocities. The predictions of this model are compared in Figure 25 to the experimental data collected by El-Shobokshy (1983) in vertical tubes with 0,7 and $20 \mu \mathrm{m}$ roughness elements, corresponding to values of $k^{+}$of approximately $0,0.5$ and 1.5 . This model consistently predicts lower deposition velocities than observed in the experiments, but the model does capture similar trends as the experiments. Large increases in deposition velocities with increases in surface roughness are seen in both the model and in the experiments. Furthermore, the importance of surface roughness in determining deposition velocity diminishes as the particle size increases in the model and in the experiments. This is especially clear in the model, where the lines for the three different roughness values converge for values of $\tau^{+}$ greater than 20 .

El-Shobokshy \& Ismail (1980) modified the model of Liu \& Ilori (1974) to account for surface roughness by changing $\Delta^{+}$using equations (92)-(94). They also included Brownian diffusion in their analysis. El-Shobokshy \& Ismail used the Lin et al. (1953) correlation for the eddy viscosity. By setting the upper integration limit at the edge of the 
turbulent core instead of the duct centerline, they were able to solve for deposition velocity without resorting to an analogy to vapor diffusion as in Liu \& Ilori. The model of El-Shobokshy \& Ismail is also displayed in Figure 25 at the three different roughness heights of the experiments. This model exhibits better agreement with the experimental data at all roughness levels than the model of Browne (1974). As with the previous model, this model seems to follow the same trends as observed in this experimental data set.

Wood (1981a) presents a form of a free-flight model that includes Brownian diffusion and is suitable for calculating deposition to rough surfaces. For the initial free-flight velocity, Wood correlated his model output with Liu \& Agarwal's (1974) experimental data to arrive at a constant value of 0.69 for $v_{p y, \Delta^{+}}^{+}$, applicable for all particle sizes. To account for rough surfaces, Wood used a similar approach as Browne (1974), where the capture distance was defined as

$$
\Delta^{+}=S^{+}+r_{p}^{+}+k^{+}-e^{+}
$$

and the offset in the velocity profile was chosen to be

$$
e^{+}=0.55 k^{+}
$$

Equation (95) is the same as (92) except that the standard deviation of the roughness height is neglected. Equation (96) is a simpler version of (94) based on the same three velocity profile offsets measured by Grass (1971). Considering the sparse data, equations (95) and (96) are probably more appropriate estimates of a particle capture distance than (92)-(94).

The proposed model of Wood (1981a) applied to the three roughness conditions in the experiments of El-Shobokshy (1983) is presented in Figure 26. Similar results as observed in Browne's (1974) model accounting for roughness is observed. The model appears to follow similar trends as the experimental data, but deposition velocities predicted by the model are somewhat lower than those measured experimentally.

Im \& Ahluwalia (1989) present a free-flight model that is mostly independent of the previously discussed models. A key feature of this model is its ability to account for variations in surface roughness. The particle eddy diffusivity was not assumed to equal the eddy viscosity; rather, a relationship between the two depending on the ratio of the 
turbulent integral time scale to the particle relaxation time was proposed. The turbulent integral time scale was defined as

$$
\tau_{f}=\frac{2 y_{b}}{3 u^{*}}
$$

where $y_{b}$ is the thickness of the buffer layer, taken to occur at $y^{+}=80$. The initial freeflight velocity of the particle was assumed to depend on the ratio $\tau_{f} / \tau_{p}$ as well. The particle capture distance was taken to be

$$
\Delta^{+}=S^{+}+e^{+}
$$

This formulation neglects particle interception and assumes that particles deposit at the same height as the origin of the velocity profile. This is the same as assuming that the particle concentration profile is offset to the same point as the velocity profile. Experimental data compiled by Cebeci \& Smith (1974) for the offset in the velocity profile versus roughness height were used to develop the following correlation

$$
e^{+}=0.7 \sqrt{k^{+}}+0.014 k^{+}
$$

This correlation yields a somewhat larger velocity profile offset than equations (94) or (96) for values of $k^{+}$less than about 2 and a smaller offset in the velocity profile for larger values of $k^{+}$.

Model results from Im \& Ahluwalia (1989) are also shown in Figure 26. The model predicts extremely low deposition velocities for small particles because it neglects Brownian diffusion. The model shows reasonable agreement with the experimental data depositing to the smooth surface and predicts deposition velocities less than observed for the case with the largest roughness. As a consequence of the scaling of the free-flight velocity and eddy diffusivity with the turbulent integral time scale, this model also predicts decreasing deposition velocity with increasing particle size in the inertiamoderated regime. This feature is not observed in any other free-flight model.

Overall, the model of El-Shobokshy \& Ismail (1980) appears to be the free-flight model that compares most favorably to the limited experimental data on deposition to rough surfaces, at least in the diffusion-impaction regime. It also displays good agreement with experimental data to smooth surfaces and includes Brownian diffusion in its analysis. However, this model arbitrarily assigns particle diffusivities greater than the eddy 
viscosity as represented by equation (91). Another weakness is that the method of accounting for roughness in this model is based on intuition and the correlation for the offset in velocity profile used to calculate the capture distance is based on only three data points, with only one of those being in the hydraulically smooth region of interest here. It is uncertain whether the model-measurement agreement is the result of the model capturing the physics of the deposition process or a mere coincidence. The model for rough surfaces presented by Browne (1974), based on the model for smooth walls proposed by Davies (1966b), seems to be the free-flight model based on the most physically sound assumptions, but this model predicts deposition velocities smaller than those measured by an order of magnitude or more. In general, free-flight theories can compare favorably to experimental measurements when they assume either seemingly unreasonably large initial free-flight velocities or large particle eddy diffusivities. When the assumptions are most realistic, the theories tend to predict deposition velocities that are much lower than observed in experiments.

\subsubsection{Gradient diffusion models}

Gradient diffusion models are similar to free-flight models in that they are solutions to the particle mass conservation equation. In fact, gradient diffusion models can be considered a conceptual simplification of free-flight models where the only deposition mechanisms included are Brownian and turbulent diffusion. Particles are assumed to diffuse from the turbulent core all the way to the wall, with no allowance made for jumping across the near-wall region of the flow by inertial coasting as in free-flight models. Thus, gradient diffusion models are free of the somewhat questionable assumption made in free-flight models that a depositing particle stops diffusing precisely at the capture distance and instantly begins its free flight to the wall.

Equation (82), Fick's law of diffusion in one dimension, is the starting point for gradient diffusion models. Nondimensionalizing this equation and solving for the dimensionless deposition velocity in the case of a smooth surface yields

$$
\frac{1}{V_{d}^{+}}=\int_{r_{p}^{+}}^{y^{+}\left(C^{+}=1\right)} \frac{d y^{+}}{\left(\frac{D_{B}}{v}+\frac{\xi_{p}}{v}\right)}
$$


The lower limit of the integral is the dimensionless particle radius, $r_{p}{ }^{+}$, because the center of a particle deposited on a smooth wall lies at a distance of the particle radius from the surface. Because diffusion is assumed to be the only deposition mechanism, no assumptions about initial free flight velocity or particle capture distance are required as in free flight models. One only needs to assume a correlation for the particle eddy diffusivity and a distance from the wall where the particle concentration is constant $\left(y^{+}\left(C^{+}=1\right)\right)$ to solve equation $(100)$.

Sehmel (1970b) used experimental deposition data in the framework of the free-flight theory to back calculate the initial free-flight velocities of depositing particles. He found that initial free-flight velocities decreased as particle size increased which is the opposite of what one would expect if free flight were indeed the means by which particles arrive at the surface. He also concluded that free-flight theories did not acceptably predict deposition velocities and he abandoned the concept of free-flight deposition in favor of deposition by gradient diffusion only. Sehmel (1970b) represents the first published non free-flight model. In this model, he proposes that the particle eddy diffusivity is greater than the eddy viscosity. By correlating the available experimental deposition data (Stavropolous, 1954; Friedlander \& Johnstone, 1957; Postma \& Schwendiman, 1960; Sehmel, 1968), he arrived at an expression for the particle eddy diffusivity for a particle depositing to a smooth vertical wall of

$$
\frac{\xi_{p}}{v}=0.011 y^{+1.1} \tau^{+^{1.1}}
$$

In applying the model, equation (101) is considered to be bounded by two limits. On the high side, the particle eddy diffusivity cannot exceed this value:

$$
\frac{\xi_{p}}{v}=0.4 y^{+}
$$

On the low side, the particle eddy diffusivity is limited by this expression:

$$
\frac{\xi_{p}}{v}=0.001 y^{+^{3}}
$$

With these equations for the particle eddy diffusivity, Sehmel (1970b) recommends integrating equation (100) from the duct centerline. This gradient diffusion model is compared to the data collected by Liu \& Agarwal (1974) in Figure 27. The model agrees with the magnitude and functional shape of the observed deposition velocities. 
Discontinuities in the model are visible and result from the abrupt changes in particle eddy diffusivity when the bounds expressed by equations (102) and (103) are reached and equation (101) is no longer applied. The proposed equations for $\xi_{p}$ have no sound theoretical support. Instead, they represent empirical fits to the data that were then available.

This type of gradient diffusion model with a particle eddy diffusivity correlation back calculated from experimental data was extended to horizontal floor and ceiling surfaces by Sehmel (1973). Correlations with a functional form similar to equation (101) were proposed for the particle eddy diffusivity of particles depositing to both floor and ceiling surfaces and bounds of applicability similar to equations (102) and (103) were also provided. Because these correlations were based on a smaller data set then that for the vertical surface, they are potentially less universally applicable.

Another gradient diffusion model that has been proposed is that of Lai \& Nazaroff (2000). This model was developed for predicting particle deposition to indoor surfaces where turbulence intensities are much smaller than in most turbulent flows considered here. In indoor environments, particle inertia is not expected to be important for determining particle transport; thus, inertia was excluded from consideration in this model. For particle deposition to a vertical surface, this work recommends integrating equation (100) from an upper bound of $y^{+}=30$ using a new correlation for the eddy viscosity based on a fit to data developed from DNS applied to channel flow (see Table 9). For deposition to horizontal floor and ceiling surfaces, a term for the flux due gravitational settling is included in the original flux equation. Thus, equation (82) becomes

$$
J=\left(D_{B}+\xi_{p}\right) \frac{d \bar{C}}{d y} \pm v_{g} \bar{C}
$$

In this work, the eddy viscosity and particle eddy diffusivity were assumed to be equal. This model, when applied to the experimental conditions of Liu \& Agarwal (1974), drastically under predicts deposition velocities for particles in the diffusion-impaction and inertia-moderated regimes, as seen in Figure 27. The eddy viscosity correlation is accurate and the model is based on physically sound assumptions if turbulent and Brownian diffusion are the only mechanisms leading to particle deposition. The fact that this model so seriously underpredicts deposition velocities to vertical surfaces in the 
diffusion-impaction and inertia-moderated regimes strongly demonstrates the importance of particle inertia in influencing deposition in these domains.

Gradient diffusion models are probably inappropriate for predicting deposition velocities in sampling tubes and ventilation ducts where air velocities are high and particles may not faithfully follow fluid streamlines. The gradient diffusion model with the best physical basis, that of Lai \& Nazaroff (2000), does not incorporate effects owing to particle inertia and consequently predicts deposition velocities far lower than are observed experimentally. The gradient diffusion model of Sehmel (1970b) shows better agreement with the experimental data, but the correlation for the particle eddy diffusivity was chosen to make the model fit the available experimental data and it is not supported by theory. The particle eddy diffusivity used in this model needs to be orders of magnitude greater than the eddy viscosity for the model to perform well. As noted earlier, several researchers have advanced strong arguments that, over long time periods, the particle eddy diffusivity should be equal to the eddy viscosity.

\subsubsection{Turbophoretic models}

Caporaloni et al. (1975) were the first investigators to recognize turbophoresis as a mechanism of particle transport. Turbophoresis was expected to be most important for transporting particles in regions very close to boundaries where turbulence is highly inhomogeneous. Caporaloni et al. suggest that the exclusion of turbophoresis from previous models is the reason that assumptions about free flight to the wall, allowing the particle to jump over the highly inhomogeneous but low intensity turbulence near the wall, were necessary to achieve agreement with the experimental data. They proposed a model accounting for turbophoresis, as well as Brownian and turbulent diffusion, which was based on a solution of the particle mass conservation equation:

$$
J=-\left(D_{B}+\xi_{p}\right) \frac{d \bar{C}}{d y}+v_{t} \bar{C}
$$

This equation is simply a restatement of equation (82) modified to account for particle flux due to a turbophoretic velocity. A simple method for calculating the turbophoretic velocity as a function of distance from the wall was provided and is repeated here

$$
v_{t}=-\tau_{p} \frac{d \overline{v_{p y}{ }^{\prime 2}}}{d y}
$$




$$
\begin{aligned}
& \overline{v_{p y}{ }^{\prime 2}}=\left(\frac{a_{o} \tau_{f}+b_{o}^{2}}{a_{o} \tau_{f}+1}\right) \overline{v^{\prime 2}} \\
& a_{o}=\frac{9 \mu}{\left(2 \rho_{p}+\rho_{a}\right) r_{p}^{2}} \\
& b_{o}=\frac{3 \rho_{a}}{2 \rho_{p}+\rho_{a}} \\
& \tau_{f}=\frac{\xi_{a}}{v^{\prime 2}}
\end{aligned}
$$

No specific correlation was suggested for the eddy viscosity, $\xi_{a}$, or for the fluid wall normal velocity fluctuations, $v^{\prime}$, as a function of the distance from the wall; however, any of those presented in this work could reasonably be used in this model. This model also ignored the particle radius in the boundary condition, thus neglecting the interception effect.

Guha (1997) and Young \& Leeming (1997) both began with the particle mass and momentum conservation equations, performed Reynolds averaging and, through modest simplifying assumptions, arrived at a set of equations that could be simultaneously solved for particle deposition velocity. The two models are essentially the same, both building on ideas first put forth by Johansen (1991), but Guha developed his equations in Cartesian coordinates and Young \& Leeming developed the equations in radial coordinates. The model published by Guha is described here and is representative of both models. The manner of the derivation lends more insight into the origin of the turbophoretic term than provided in Caporaloni et al. (1975). The dimensionless form of the particle mass conservation equation that Guha arrived at was

$$
V_{d}^{+}=-\left(\frac{D_{B}+\xi_{p}}{v}\right) \frac{\partial C^{+}}{\partial y^{+}}+v_{p c y}^{+} C^{+}-C^{+} \frac{D_{T}}{v} \frac{\partial(\ln T)}{\partial y^{+}}
$$

where $v_{p c y}^{+}$is the dimensionless particle convective velocity in the $y$-direction (normal to the wall) and $D_{T}$ is the temperature-gradient-dependent diffusion constant. The particle convective velocity in the second term on the right hand side (RHS) of (111) allows for differences between the particle velocity and the local fluid velocity. This convective velocity may result from a turbophoretic force, but it may also arise from a shear-induced lift force or an electrical force, and is thus more general than the second term on the RHS of (105), which is due solely to turbophoresis. The third term on the RHS of (111) allows for particle transport by thermophoresis. 
A key advance of the work by Guha (1997) was the inclusion of the particle momentum equation, which provides a rigorous means of calculating the particle convective velocity. The dimensionless forms of the $x$-and $y$-momentum equations, as presented in that work, are

$$
\begin{array}{ll}
x \text {-momentum: } & v_{p c y}^{+} \frac{\partial v_{p x}^{+}}{\partial y^{+}}=\frac{1}{\tau^{+}}\left(u^{+}-v_{p x}^{+}\right)+g^{+} \\
y \text {-momentum: } & v_{p c y}^{+} \frac{\partial v_{p c y}^{+}}{\partial y^{+}}+\frac{v_{p c y}^{+}}{\tau^{+}}=-\frac{\partial v_{p y}^{+2}}{\partial y^{+}}+F_{l}^{+}+F_{e}^{+}
\end{array}
$$

Here, $u^{+}$is the dimensionless axial fluid velocity, $v_{p x}^{+}$is the dimensionless axial particle velocity and $v_{p y}^{+}$is the dimensionless wall-normal particle velocity (equal to the sum of the dimensionless particle diffusive and convective velocities). The $y$-momentum equation allows for the inclusion of the shear-induced lift force and an electrical force. The gravitational force is included as a positive term in the $x$-momentum equation here, indicating a vertically downward airflow. Depending on the orientation of the flow and the deposition surface, the term for gravity may have the opposite sign or be included in the $y$-momentum equation for accurate accounting of the force. The first term on the RHS of equation (113) has the same form as the turbophoretic term from Caporaloni et al. (1975) in equation (106). Guha presents a similar model as expressed in (107) to relate the fluctuating particle velocity to the local fluctuating velocity of the fluid

$$
v_{p y}{ }^{\prime 2}=\left(\frac{1}{1+0.7\left(\tau_{p} / \tau_{f}\right)}\right) v^{\prime 2}
$$

where $\tau_{f}$ is calculated by equation (110). This is a difficult quantity to represent because the particle velocity often may not directly relate to the local air velocity.

Guha adopted the simple relationship proposed by Wood (1981a) to account for both interception and surface roughness, thus the wall boundary condition for the particle mass conservation equation is evaluated at

$$
y_{o}^{+}=r_{p}^{+}+k^{+}-e^{+}=r_{p}^{+}+0.45 k^{+}
$$

This model assumes that the particle eddy diffusivity is equal to the eddy viscosity. 
Application of this model requires simultaneous numerical solution of equations (111)(113). This is a more difficult task than solution of the previously presented free-flight or gradient diffusion models, but current computing power allows for solution in just a few seconds of CPU time. Neglecting the lift force decouples equation (113) from equations (111) and (112) and allows for a somewhat easier solution of particle deposition velocities. This model is attractive because it is applicable in all particle deposition regimes and it offers the capability of including a variety of forces, including the turbophoretic force, which had not been rigorously applied in any previous Eulerian model.

To illustrate the importance of turbophoresis in determining the deposition velocity of certain sized particles, predictions from the model of Guha (1997) are plotted in Figure 28 while retaining and excluding the particle convective velocity term in equation (111). In this model application, the deposition surface was vertical and smooth, and thermal, electrical and shear-induced lift forces were ignored so that the only phenomenon contributing to the particle convective velocity was turbophoresis. The data of Liu \& Agarwal (1974) are also shown in the figure. In the case when turbophoresis is ignored, equation (111) becomes nearly identical to the gradient diffusion model of Lai \& Nazaroff (2000) and the model predicts deposition velocities that are too low in the diffusion-impaction and inertia-moderated regimes. The inclusion of the turbophoretic term leads to the prediction of a dramatic increase in deposition velocity in the diffusionimpaction regime and of a plateau and decrease in deposition velocity as particle size increases through the inertia-moderated regime. This predicted trend is in much better agreement with the observed data than most other theoretically based models, although the agreement with the experimental data is not perfect.

Figure 29 again compares the model of Guha (1997) to the experimental data of Liu \& Agarwal (1974). In this case, the model was applied with three different deposition surface roughnesses of 0,5 and $20 \mu \mathrm{m}$, corresponding to dimensionless roughness values of 0.25 and 1.0. The model line for $k^{+}=0.25$ agrees well with the data across the entire range of particle sizes. More than one investigator has suggested that small amounts of surface roughness in the experiments of Liu \& Agarwal led to measured deposition velocities that were slightly enhanced compared to those that would have occurred if the surface had been truly smooth (Wood, 1981a; Guha, 1997). However, this level of roughness probably overstates the level that was present in these experiments, owing to the smooth nature of the glass deposition tubes utilized. Other modeling efforts with this 
type of model (Johansen, 1991) suggest that relatively small temperature gradients or the presence of an electric charge on the particles during the experiments could account for the discrepancy in deposition velocities between model and measurement at the smallest particle sizes studied.

Young \& Leeming (1997) used their model to analyze predicted airborne particle concentration profiles near the deposition surface. They found that particles in the diffusion-impaction regime were predicted to have a maximum in the concentration profile very near a vertical wall. This finding is consistent with the accumulation of particles of this size in the near-wall region predicted by many Lagrangian simulations (i.e. Brooke et al., 1994). Turbophoretic models are the only Eulerian models that predict this near-wall accumulation of particles.

Turbophoretic models of the form presented in Guha (1997) and Young \& Leeming (1997) are probably the Eulerian models that are best suited to predicting particle deposition in ventilation ducts. These models faithfully reproduce trends observed in experimental data and give reasonably good predictions of absolute deposition.

Furthermore, these models are applicable across the entire size range of particles and they are physically satisfying, with only a small amount of empiricism. Such models can account for a wide variety of forces that may act on particles. They also show good agreement with deposition velocities and concentration profiles predicted by the best Lagrangian simulations, but at a much lower computational cost. These models are limited to application in fully developed turbulent flow. Changes in deposition rates owing to the presence of flow disturbances and surface irregularities are not predicted by these models.

\subsection{Sublayer models}

Sublayer models use a Lagrangian scheme to calculate particle trajectories in the nearwall region of a flow and thereby predict particle deposition velocities. However, these models are different than fully Lagrangian simulations that calculate trajectories for large numbers of particles. In sublayer models, a single limiting or critical trajectory in the near-wall region is calculated for a particle that just impacts the surface. Predicted deposition velocities are based on the percentage of trajectories that would bring particles into closer contact with the wall than the limiting trajectory. These models are termed sublayer models because limiting particle trajectories are calculated only for the near- 
wall flow region, sometimes called the sublayer. Owen (1969) commented on the weaknesses of free flight models and proposed '...that particles are convected to the wall from the region of energetic turbulent motion outside the viscous sublayer by the occasional large eddy that encroaches on it...'. This proposition was based on the then recent discovery of turbulent bursts, down sweeps and coherent structures in near-wall turbulence described by Kline et al. (1967).

Three groups of researchers have proposed sublayer models and all follow the same general scheme. The coherent near-wall turbulence described by Kline et al. (1967) and others is modeled as a two-dimensional, stagnation-point flow as described in Schlichting (1979). Figure 30 provides a schematic description of such flow in the $y$-z plane, normal to the main flow direction. Flow is directed towards the wall near a centerline that passes through a stagnation point and is deflected away from the wall further from the stagnation point. As described earlier, these structures repeat at regular intervals with a typical spacing of approximately $\lambda_{z}^{+}=100$. The region outside that shown in the figure is assumed to have a uniform particle concentration. A particle entering the near-wall region from the well-mixed region is assumed to deposit to the wall if its initial spanwise location is closer to the centerline than the initial location of the limiting trajectory. Conversely, it is swept back up into the well-mixed core if the starting position is further from the centerline than the limiting trajectory. Particle trajectories are calculated by assuming an initial position and solving the particle equations of motion with the goal of determining the limiting particle trajectory. The limiting particle trajectory is defined as that trajectory which leads to the deposition of the particle at a distance of $\lambda_{z} / 4$ from the centerline. A particle following the limiting trajectory and depositing on a smooth wall is shown in Figure 30. Once the limiting trajectory is determined, the dimensionless deposition velocity for inertial particles can be calculated from the initial spanwise particle location of this trajectory, $z_{\text {lim }}$, and the initial particle velocity towards the wall at this location, $v_{p y, o}$, via

$$
V_{d, \text { inertial }}^{+}=\frac{2 z_{\lim }^{+} v_{p y, o}^{+}}{\lambda_{z}^{+}}
$$

This basic approach of determining a critical trajectory to predict particle deposition rates is common in other applications, such as sedimentation basins for water and wastewater treatment, particle impactors and laminar-flow electrostatic precipitators. For particles 
where Brownian diffusion is significant, this method is invalid and a different approach that includes Brownian diffusion is necessary.

Cleaver \& Yates (1975) were the first investigators to propose a mathematical form of a sublayer model for smooth surfaces. Their model assumed that the boundary between the turbulent core and the sublayer occurred at $y^{+}=10$ and particles were assumed to enter the sublayer with an initial dimensionless velocity, $v_{p y, o}^{+}$, of 0.5 . In the particle momentum equations used to calculate the limiting trajectory, the drag force was assumed to be the only force acting on the particle. Cleaver \& Yates applied their model with two different sublayer flow models, the two-dimensional stagnation flow model described above and a flow model based on the experimental data of Laufer (1954). They found little difference between the model predictions. The basic model was found to predict deposition velocities lower than measured in experiments and the model was modified to account for axial convection into regions with down sweeping flow. The manifestation of this modification was an ad hoc factor to improve the modelmeasurement agreement and it has weak theoretical support. Cleaver \& Yates performed a separate analysis for deposition of particles when Brownian diffusion is important. They solved the diffusion equation for stagnation point flow and, through some simplifying assumptions, arrived at this expression for diffusional deposition velocity:

$$
V_{d, d i f f}^{+}=0.084 \mathrm{Sc}^{-2 / 3}
$$

This expression has the same form as the empirical equation (52) for the diffusive deposition regime. Cleaver \& Yates suggested that the deposition velocity of all particles could be predicted by adding the inertial and diffusive components of deposition:

$$
V_{d}^{+}=V_{d, \text { inertial }}^{+}+V_{d, \text { diff }}^{+}
$$

One of the results reported in this model is the strong influence of the particle to fluid density ratio on deposition velocity when plotted in the form of $V_{d}^{+}$versus $\tau^{+}$. This has repeatedly been shown in subsequent investigations, and it is believed that Cleaver $\&$ Yates were the first to recognize the importance of this density ratio.

Fichman et al. (1988) proposed a sublayer model with particle momentum equations that included both drag and the shear-induced lift force. The sublayer-turbulent core boundary was assumed to be at $y^{+}=30$ and the initial dimensionless particle velocity was taken as 0.8 . Correlations were developed to model the two-dimensional stagnation flow. 
Fichman et al. found good agreement between their model and the experimental data and they attributed the improvement over previous models to the inclusion of the shearinduced lift force. The lift force was noted to be especially important for particles in the diffusion-impaction regime very close to the wall $\left(y^{+}<10\right)$. Application of the model as presented in this review is made impractical owing to what seem to be errors in the reporting of constants used in the solution of the particle momentum equations. The same difficulty with this model has been reported by Fan \& Ahmadi (1993).

The most well-developed sublayer model in the literature is that of Fan \& Ahmadi (1993), which includes drag, shear-induced lift and gravitational forces in the particle momentum equations. The boundary condition for a deposited particle was modified to account for surface roughness following the method of Browne (1974). In this model, the interface between the turbulent core and the sublayer was assumed to occur at $y^{+}=12$ and the initial dimensionless particle velocity upon entering the sublayer was assumed to be 0.7 . This value of $v_{p y, o}^{+}$is somewhat higher than experimentally measured values at this distance from the wall (0.3-0.5), and the higher value was rationalized by the fact that particles near the centerline of a down sweep will tend to have a higher wall-normal velocity than the time-averaged value over all locations. The sublayer stagnation flow was approximated using the model presented in Schlichting (1979). Fan \& Ahmadi adopted the method of Cleaver \& Yates (1975) for particles with significant rates of Brownian diffusion; accordingly, the total deposition velocity was calculated using equations (116)-(118).

The model of Fan \& Ahmadi (1993) is applied to the conditions of the experiments by Liu \& Agarwal (1974) in Figure 31. The model for deposition to a vertical wall is applied assuming three different values for the surface roughness, 0,5 and $20 \mu \mathrm{m}$. The model predicts the general shape of the data reasonably well, although for deposition to the smooth surface in the range of $1<\tau^{+}<10$, it predicts an increase in deposition velocity that is sharper than observed experimentally. In the diffusion-impaction regime, the model for the smooth surface underpredicts deposition velocities for particles with $\tau^{+}$ $<4$ and over predicts for $\tau^{+}>4$. The inaccurate sharpness of the model may derive from representing the turbulence structure as uniform based on its mean properties, rather than variable with a distribution of properties. If deposition is more strongly influenced by turbulence events that are more intense than the average, models that are based on mean turbulence properties will give unreliable predictions. Dimensionless deposition velocities are predicted to increase dramatically in the diffusion and diffusion-impaction 
regimes with increases in surface roughness. Again, as observed in previous models, small amounts of roughness buried in the laminar sublayer are predicted to strongly enhance deposition rates. This consistent modeling outcome for different types of models could be anticipated since the method of accounting for the surface roughness in all the models is nearly identical.

Fan \& Ahmadi (1993) also provide an empirical expression based on a perturbation solution that closely reproduces the deposition predictions of the full sublayer model, including the effects of surface roughness and shear-induced lift. Fan \& Ahmadi (1994) and Fan \& Ahmadi (1995), respectively, extended their sublayer model to account for electrophoresis and nonspherical particles.

Sublayer models are not fully Lagrangian models; a small number of trajectories of a given sized particle are calculated until the limiting particle trajectory is found. Because deposition velocities are calculated based on the limiting particle trajectory and not on statistics of a large ensemble of particles, the computational requirements are significantly lower than fully Lagrangian models and sublayer models may be solved in just a few seconds of CPU time with current computational power. These models attempt to capture the interaction between particles and near-wall turbulent eddies that impinge on the deposition surface. From investigations of the near-wall turbulence structure and Lagrangian simulations of particle deposition, these eddies are believed to play a key role in the deposition of particles, especially those in the diffusion-impaction regime. Sublayer models reproduce the shape of the deposition curve observed in experiments well with physically satisfying assumptions. The sublayer model of Fan \& Ahmadi (1993) is probably the most appropriate model of this type for application to the case of particle deposition in ventilation ducts.

\subsection{Lagrangian simulations}

The general approach to investigating particle deposition in turbulent flow by Lagrangian simulation involves two steps. First, the flow field is mathematically described, and second, particles are released into the simulated flow field and trajectories are tracked based on the prescribed equations of particle motion. It is usually assumed that the presence of particles in the fluid does not affect the structure of the turbulent flow. This assumption is termed the one-way coupling assumption, referring to the fact that the fluid affects the particle momentum, but the particles do not influence the momentum of the 
fluid. This assumption is reasonable when the mass concentration of suspended particles is very much smaller than the air density, as would be true in ventilation ducts.

Lagrangian models discussed here are grouped by the method used for generating the turbulent flow field. Lagrangian simulations utilizing simpler stochastic turbulence models are discussed first in Section 4.6.1, followed by simulations that use LES and DNS to generate the description of turbulent flow in Section 4.6.2.

\subsubsection{Lagrangian simulations with stochastically modeled turbulent flow}

A summary of the flow conditions and assumptions used in the Lagrangian simulations discussed in this section is given in Table 14. The earliest works in this area (Hutchinson et al., 1971, and Reeks \& Skyrme, 1976) are not included in Table 14. These early works were developed as predictive models to be applied to a variety of conditions. They comprised composites of Lagrangian simulations and Eulerian models and were not 'numerical experiments' of the type represented by more recent Lagrangian simulations.

It should be noted that turbulent diffusion and turbophoresis do not appear in the 'Forces' column of Table 14. These phenomena must be included in Eulerian models to capture the interactions between particles and turbulent eddies; in Lagrangian simulations, these particle-eddy interactions are directly calculated. Thus, particle drift owing to turbulent diffusion and turbophoresis are captured to some degree in all Lagrangian simulations.

The first particle deposition model to incorporate a Lagrangian framework (Hutchinson et $a l ., 1971)$ was rather crude. This model was divided into two sections: a Lagrangian random walk trajectory in the plane normal to the main flow was calculated to determine the fraction of particles that approach a stagnant wall layer and then an Eulerian calculation, similar in concept to the free-flight models, was used to calculate the fraction of those particles approaching the wall layer that actually traverse the layer and deposit. When multiplied together, these two fractions yield the fraction of the total particles in

the system that deposit. The stagnant wall layer was assumed to have a thickness of $y^{+}=$ 1.25 to best fit the experimental data.

Hutchinson et al. (1971) assumed that steps taken in the random walk were the result of interactions with large, energy-containing turbulent eddies and only the drag force was taken into account. Relationships for the length and time scales associated with these eddies were taken from the work of Townsend (1956) and Laufer (1954). The direction 
of each step was randomly assigned; thus, the likely correlation between successive steps in time was ignored. The fraction of particles approaching the stagnant wall layer that actually deposit was determined by integrating the particle velocity density distribution function over all possible initial particle velocities and approach angles to determine the fraction of particles of that size with sufficient inertia to reach the wall by free flight. A Stokesian drag force was the only force considered in this Eulerian portion of the model. Reasonable agreement with the available data was reported in this work; however, the wall-layer thickness was empirically adjusted to achieve the best fit to the data.

Reeks \& Skyrme (1976) followed the same procedure outlined in Hutchinson et al. (1971). However, instead of solving for a random-walk trajectory to determine the fraction of particles approaching the stagnant wall region, the particle flux toward the wall region was obtained through integration of the particle velocity distribution function that was assumed to be jointly normal. Calculation of the fraction of these particles approaching the wall that actually deposit was carried out as in Hutchinson et al., except that the stagnant wall layer was assumed to begin at a distance from the wall of $y^{+}=5$, instead of $y^{+}=1.25$. The model of Reeks \& Skyrme was developed for large particles in the inertia-moderated regime and at the high end of the diffusion-impaction regime. The model predicts an extremely rapid increase in deposition velocity as particle size increases through the diffusion-impaction regime, one that is much larger than observed experimentally or predicted by any other model. The model does successfully capture the magnitude of the deposition velocity and the experimentally observed decrease in deposition velocity with increasing particle size in the inertia-moderated regime. However, this agreement with the experimental data was facilitated by the empirical adjustment of two model parameters.

The first fully Lagrangian simulation of particle deposition from turbulent flow is presented by Kallio \& Reeks (1989). In this study, and in those that have followed, the deposition velocity of a given sized particle is determined from the calculated trajectories of thousands of particles. The turbulent flow in this work was modeled as a twodimensional random velocity field. Profiles of mean and fluctuating air velocities were fit to the experimental results of Laufer (1954) and others. The particle trajectory was determined from repeated interactions with discrete turbulent eddies. Each turbulent eddy was assumed to have a random wall-normal velocity component drawn from a Gaussian distribution and a random time scale drawn from an exponential distribution. Particles were assumed to interact with eddies for a length of time equal to the eddy time 
scale. The particle momentum equations included the lift force. The lift force was not included in some simulations so that its influence on deposition rates could be evaluated.

The results of the simulation of Kallio \& Reeks (1989) are plotted along with the data of Liu \& Agarwal (1974) in Figure 32. Details such as the air velocity and the duct diameter for the simulated flow were not reported by Kallio \& Reeks. This relatively simple simulation agrees well with the experimental data on several points. The magnitudes of the simulated deposition velocities are similar to those that were experimentally observed, the slopes of the deposition curve in the diffusion-impaction regime are similar in both cases, and the decrease in deposition velocity at high values of $\tau^{+}$observed in experiments is also observed in the simulation. This simulation also suggests that the lift force is most important for particles with dimensionless relaxation times in the range $1<\tau^{+}<20$. However, the authors caution that the effect of the lift force is only approximate, because the restrictions on the equation for lift force derived by Saffman $(1965,1968)$ were frequently violated and because the equation is not strictly applicable near a wall. Another observation in this simulation was the accumulation of particles in the diffusion-impaction regime near the vertical wall, in the region $0.1<y^{+}<$ 3. This increase in the particle concentration profile near the wall is similar to that predicted by means of the turbophoretic model of Young \& Leeming (1997).

Li \& Ahmadi (1993a) performed a particle deposition simulation where Brownian diffusion was simulated as a Gaussian white-noise process and the particle momentum equations included the effects of drag, shear-induced lift and gravity. The turbulent velocity field was generated from a Gaussian random field that was modified to account for the anisotropic nature of the turbulence near the wall. Another novel aspect of this simulation was that it allowed particles striking the wall to either deposit or to bounce depending on the energy of the particle-surface collision. Particles striking the surface with a velocity greater than a critical velocity were assumed to bounce, while those with a lesser velocity deposited. The critical velocity was defined by

$$
v_{p, c r i t}=\sqrt{\frac{2 E_{o}}{m_{p}}\left(\frac{1-r^{2}}{r^{2}}\right)}
$$

where $m_{p}$ is the mass of the particle, $r$ is the restitution coefficient and $E_{o}$ is the potential energy of the surface. The restitution coefficient ranges from zero to one, with a value of 
one indicating a perfectly reflecting surface. The surface potential energy was calculated by

$$
E_{o}=\frac{A_{H} d_{p}}{12 y_{e}}
$$

where $y_{e}$ is the equilibrium separation between a particle and a surface (recommended as $0.4 \mathrm{~nm})$ and $A_{H}$ is the material dependent Hamaker constant for the particle-surface interaction (on the order of $10^{-19} \mathrm{~J}$ ).

Li \& Ahmadi (1993a) performed simulations in $2 \mathrm{~cm}$ wide vertical and horizontal channels with an air velocity of $5 \mathrm{~m} / \mathrm{s}$ and a friction velocity of $30 \mathrm{~cm} / \mathrm{s}$. Results for particles of two different densities depositing to a vertical wall are displayed in Figure 33 along with Liu \& Agarwal's (1974) data. The results from the simulation lie somewhat below the experimental data for both particle densities. This simulation does capture the shape of the deposition curve seen in experiments across the entire range of particle sizes. Only a small difference is observed for particles of different densities. Figure 34 shows the results of Li \& Ahmadi's simulation to a horizontal floor surface. Simulated deposition rates to the horizontal surface are much greater than those to the vertical surface owing to the effects of gravitational settling. The results compare favorably with the experimental findings of Sehmel (1973) who collected experimental data at a similar friction velocity of $34.1 \mathrm{~cm} / \mathrm{s}$. The simulation results for all but the smallest particles also agree with the line representing the expected deposition velocity if gravitational settling were the only particle transport mechanism. Li \& Ahmadi also simulated deposition in cases where particles were allowed to bounce after impact depending on the energy of the collision. The results of these investigations, shown in Figure 35, suggest that particle bouncing upon wall impact is only important for $\tau^{+}>10$, (i.e., for particles in the inertiamoderated regime). As observed in other Lagrangian simulations and as predicted by turbophoretic models, an accumulation of particles in the diffusion-impaction regime very close to the vertical wall was observed in this simulation. Such a concentration profile was not observed in the case of the horizontal floor surface owing to the influence of gravity.

Li \& Ahmadi (1993b) used the same procedure as Li \& Ahmadi (1993a) to investigate the role of surface roughness in particle deposition to vertical walls and horizontal floors. In this case, the condition for particle deposition was modified for rough surfaces by the 
method described in Wood (1981a) (equations 95 and 96) and particle bounce was ignored. To our knowledge, this is the only Lagrangian simulation of particle deposition to include the effect of surface roughness. Results of the simulation considering deposition to a vertical wall with four different surface roughness values are shown along with the experimental data of El-Shobokshy (1983) in Figure 36. In this simulation, increasing surface roughness is observed to dramatically increase deposition velocities for smaller particles, and the effect is observed to decrease as particle size increases. These are the same trends seen in the data of El-Shobokshy and predicted by all Eulerian models that account for surface roughness. The Li \& Ahmadi simulation yields lower values of deposition velocity than the experimental data, a similar situation as observed with most Eulerian models. As in previous simulations with smooth vertical walls, an accumulation of particles in the diffusion-impaction regime was observed in the nearwall region of the rough vertical walls, although the effect was less dramatic in the rough wall case than in the smooth wall simulation. The Li \& Ahmadi (1993b) simulation results for deposition to rough horizontal floor surfaces are shown in Figure 37. The impact of roughness on deposition velocity is observed to be much less in this case than was observed in the vertical wall simulation. No difference in deposition velocity is seen for particles with $\tau^{+}>0.1$ because gravitational settling is the dominant deposition mechanism in this case and it is relatively unaffected by changes in surface roughness.

Li et al. (1994) used a similar method as in Li \& Ahmadi (1993a) to examine deposition in a channel at a $12.5 \mathrm{~mm}$ wide (streamwise) by $6.1 \mathrm{~mm}$ tall rectangular obstruction and for a more streamlined trapezoidal obstruction of similar dimensions. Deposition to the three exposed faces of the obstructions was evaluated and the presence of the obstructions was found to significantly enhance particle deposition, especially for larger particles, owing to impaction. The upstream face of the obstructions experienced the greatest amount of deposition and the enhancement in deposition compared to that on the flat channel surface was less for the more streamlined trapezoidal obstruction. While the results of this work are not directly comparable to other experiments or models in this paper, they are an indication of the potential importance of flow obstructions in enhancing particle deposition.

Chen \& Ahmadi (1997) performed Lagrangian simulations following a procedure similar to Li \& Ahmadi (1993a). Transport from turbulence interactions, Brownian diffusion, gravitational settling and shear-induced lift were all included. In this case, however, the flow configuration was a pipe instead of a channel and the modified equation for the 
shear-induced lift force recommended by McLaughlin (1993) was implemented. This optimum lift force alleviated the constraints imposed on equation (38) and also corrected that expression for the presence of a wall. Because the shear-induced lift force depends on the difference between the particle and fluid axial velocities, it has been argued (Fan \& Ahmadi, 1993) that the direction of flow in a vertical system could affect particle deposition velocities through the lift force. An upward flow scheme should cause the particle to lag the fluid in the axial direction, leading to a lift force away from the wall and reducing deposition, while a downward flow scheme should have the opposite effect. A goal of these simulations was to evaluate the magnitude of this effect. Figure 38 shows the results of the simulations of Chen \& Ahmadi for two cases of downward flow, one in the absence of gravity and one including gravity, which should increase the magnitude of the lift force. The difference between the two cases is small for most particle sizes and this difference is less than predicted by previous models that considered this effect. The authors attribute the lesser effect observed in their simulation to the use of the modified equation for the shear-induced lift force, which is smaller than the force calculated by equation (38) and applied in most other models.

\subsubsection{Lagrangian simulations with turbulent flow from LES and DNS}

Table 15 summarizes the conditions and assumptions made in the Lagrangian particle simulations discussed in this section. Lagrangian simulations provide details of particle trajectories that allow for the extraction of a wealth of information on their behavior in turbulent flows. Not all the simulations included in Table 15 are directly concerned with particle deposition, but all provide information on the behavior of particles in air turbulence that gives insight into the particle deposition process. Values in the table are both as presented in the cited articles and as calculated by the current authors. In cases of a channel flow configuration, $D_{h}$ refers to the full height of the channel. Frequently, authors of these studies report channel heights in dimensionless units without reference to a friction velocity for converting to a height with dimensions. As a final introductory note, in studies that allowed for the lift force and gravity, these forces were not included in every simulation; commonly, the authors would run identical simulations with and without one of these forces to illustrate the effect of that force on particle deposition.

The first Lagrangian simulation of particle deposition using a flow field generated by DNS was that of McLaughlin (1989) who studied the deposition of particles with values of $\tau^{+}$ranging from 2-6 in a simulated vertical channel flow with $\operatorname{Re}=4000$. This 
pioneering work yielded deposition velocities similar to those measured by experiment, as illustrated in Figure 39. McLaughlin introduced several new ideas that were expanded upon by subsequent investigators. In particular, McLaughlin concluded that particles were brought to the wall by strong, well-organized fluid motions. An accumulation of particles in the diffusion-impaction regime near the wall $\left(y^{+}<5\right)$ was identified such that the concentration near the wall was about twice the core concentration. Particle velocities were observed to lag the fluid velocities in both the streamwise and wallnormal directions in the turbulent core, but to lead the fluid in both directions very near the wall. Depositing particles impacted the wall with normal velocities on the order of $0.5 u^{*}$, suggesting an inertial deposition mechanism rather than a diffusive one. Also noted was the potential importance of the lift force on the particle trajectory in the nearwall region. McLaughlin suggested that the main limitations of this simulation were associated with the particle momentum equation. The particle Reynolds number conditions for both the Stokes drag force and the shear-induced lift force, equation (38), were violated by the particles in the simulations. Deposition velocities from this simulation agreed well with the experimental data over the limited range of dimensionless relaxation times studied; furthermore, this work provided information on particle Reynolds numbers, particle velocities and near wall particle concentrations that were unattainable by experiment or previous Eulerian modeling.

Ounis et al. (1991) made a significant advance in Lagrangian simulations when they included a method to account for particle transport by Brownian diffusion. Ounis et al. (1991) and Ounis et al. (1993) simulated the motion of 0.01-0.1 $\mu \mathrm{m}$ particles in the nearwall region of a DNS-generated flow by implementing a Gaussian white-noise process for the Brownian force. These simulations indicated that Brownian motion significantly affects particle behavior near the wall for particle diameters less than $0.05 \mu \mathrm{m}$ and that turbulence significantly affects deposition rates of particles with diameters larger than $0.03 \mu \mathrm{m}$. Of particular note in this simulation was that the initial locations of depositing particles that were not affected by Brownian motion were concentrated in bands where coherent vortices form strong streams toward the wall. These bands in initial particle location were separated by 100-150 wall units and corresponded to locations where nearwall vortices caused strong flows towards the wall. This is strong evidence that these vortices are responsible for the deposition of inertial particles. For particles strongly influenced by Brownian diffusion, no such patterns in initial location were observed for depositing particles. The resulting deposition velocities of these $0.01-0.1 \mu \mathrm{m}$ particles from Ounis et al. (1993) are compared to the empirical equation (52) with $k_{1}=0.084$ for 
particles in the diffusion regime in Figure 40. Note that limits of the relaxation time in this figure are different than in most others in this review, the low relaxation times in this study are the result of the small particle sizes and the low friction velocity, $3.7 \mathrm{~cm} / \mathrm{s}$. The simulation results are in close agreement with the predictions of the empirical equation.

Brooke et al. (1992) and Brooke et al. (1994) conducted Lagrangian particle tracking in a DNS-generated vertical channel flow with particles in the diffusion-impaction regime considering only the drag force. The paper by Brooke et al. (1992) contains observations similar to those reported in other simulation work. These include the accumulation of particles in the diffusion-impaction regime near the wall and their segregation into the low-speed streaks in this region, the violation of the particle Reynolds number criteria for drag calculation, the importance of near-wall vortices in causing particle deposition and the high impact velocities of depositing particles, of the same order as the friction velocity. While these high impact velocities were consistent with the high initial freeflight velocities required in free-flight theories, turbophoresis, and not turbulent diffusion, was suggested as the mechanism by which particle arrive at their capture distance.

Brooke et al. (1994) took a detailed look at particle velocities in the near-wall region and found that a small fraction, defined as free-flight particles, had a velocity much greater than, and disengaged from, the local fluid velocity. Particles with velocities similar to the local fluid velocity were defined as entrained particles. Free flight was determined to be significant only near the wall $\left(y^{+}<20\right)$ and these free-flight particles were much more likely to deposit than entrained particles. The most likely distance from which a depositing particle would begin free flight to the wall was found to be $y^{+} \sim 7-9$, regardless of the relaxation time. This location corresponded to an inflection point in the profile of the root-mean-square of the wall-normal air velocity fluctuations in this simulation. The turbophoretic force would be expected to be appreciable at this location owing to the steep gradient in turbulence intensity at this location. Brooke et al. (1994) also analyzed trajectories of particles released very near the wall, at $y^{+}<3$. These particles were found to rarely deposited and, when they did, they usually first escaped the near-wall region and initiated a free flight to the wall from a greater distance.

Chen \& McLaughlin (1995) performed a simulation in a DNS-generated vertical-channel flow using a particle equation of motion with the wall-corrected drag force and the optimum lift force (lift corrected for both the presence of a wall and for high Reynolds number effects). Extremely large accumulations of particles in the diffusion-impaction regime were observed for $y^{+}<1$; a value of $C^{+}=250$ was observed for particles with $\tau^{+}=$ 
10 in this near-wall region. The deposition velocities observed in this simulation lie below those measured in the experiments of Liu \& Agarwal (1974) as seen in Figure 41. Deposition rates in this simulation are lower than observed in other numerical simulations as well, possibly due to the decreased lift force and increased drag force near the wall. Chen \& McLaughlin developed an empirical fit to their simulation results to look at the effects of particle polydispersity on deposition rates and suggested that very small amounts of polydispersity can lead to large changes in measured deposition rates in experiments. Particle polydispersity is cited as a likely cause of the wide scatter in the data of deposition experiments.

Wang \& Squires (1996a) and Wang \& Squires (1996b) demonstrated the feasibility of particle simulations in LES-generated channel flows. Because of the enormous computational intensity required, DNS is limited to low flow Reynolds numbers and short simulation times. The LES-method was used to increase the Reynolds numbers in simulations and to simulate particles with long relaxation times that are currently not amenable to analysis by DNS. These advantages in LES are achieved by the less accurate accounting of small-scale near-wall turbulent eddies. Wang \& Squires (1996a) observed similar particle velocity statistics and particle concentration profiles in simulations in flows generated by LES and DNS. As seen in simulations using DNS, particles in the diffusion-impaction regime accumulating near the wall were found to segregate preferentially in the low-speed, low-vorticity streaks. Wang \& Squires (1996b) compared particle simulations in an LES-generated vertical-channel flow at Reynolds numbers of 11,160 and 79,400 to the DNS results of McLaughlin (1989). The comparison of LES and DNS was favorable and features of particle behavior commonly observed in simulations with DNS were also observed in this LES. It was noted that the LES results deviated most from the DNS results for smaller particles that are most sensitive to the smallest eddies, which are modeled approximately in LES but are completely resolved in DNS. The deposition profile from the simulation of Wang \& Squires (1996b) at $\operatorname{Re}=11,160$ is shown in Figure 42 for cases where the lift force was either included or excluded. The results of this simulation agree well with the experimental data, and with previous DNS results. Both simulations predicted a steeper increase in $V_{d}^{+}$with $\tau^{+}$in the diffusion-impaction regime than what has been observed experimentally, but the magnitude of deposition in the simulations is in accord with experiments. Almost no difference was seen in this deposition profile when the Reynolds number was increased from 11,160 to 79,400 . In general, these works showed LES to be 
a useful tool in analyzing particle deposition in larger Reynolds number flows and for larger values of $\tau^{+}$than currently can be handled by DNS.

Uijttewaal \& Oliemans (1996) performed simulations of particles in the inertiamoderated regime in vertical cylindrical-tube flows generated by both DNS and LES. DNS was used to generate a flow with $\operatorname{Re}=5300$, and LES was used to generate flows with Reynolds numbers of 18,300 and 42,000 . Due to the large particle sizes in these simulations, the Stokes drag force was modified to account for large particle Reynolds numbers. Many of the results commonly reported in DNS particle transport simulations were observed in these LES simulations: an accumulation of particles in the near-wall region in the diffusion-impaction regime; and large particle Reynolds numbers and large impact velocities when depositing to a wall, suggesting a nondiffusive deposition mechanism. Deposition velocities of particles in these simulations are compared to the experiments of Liu \& Agarwal (1974) in Figure 43. Note that Figure 43 extends further into the inertia-moderated regime than most figures presented in this report. The simulation results match those of the experiments in absolute magnitude and in the trend of decreasing deposition velocity with increasing particle size in the inertia-moderated regime. Simulated deposition velocities were also found to increase with increasing Reynolds number at the highest relaxation times. Because these particles are so large, their motion is not significantly influenced by flows in the near-wall region. Instead it is dominated by the larger eddies in the turbulent core that scale with the Reynolds number. While concentration profiles of particles in the diffusion-impaction regime showed an accumulation of particles immediately adjacent to the wall, no such accumulation was observed for particles in the inertia-moderated regime.

Wang et al. (1997) calculated trajectories incorporating the optimum lift force in the particle equations of motion in the same LES channel flows described in Wang \& Squires (1996b). Figure 44 displays the results. Deposition velocities when the optimum lift was included in the calculations were higher than in the case with no lift for particles with $2<$ $\tau^{+}<20$, but less than previously observed when the unmodified lift expression was utilized (Wang \& Squires, 1996b). Very little difference was observed for the deposition velocities at two different Reynolds numbers $(11,160$ and 79,400$)$. The deposition velocities predicted in these simulations are less than the corresponding experimental measurements of Liu \& Agarwal (1974). The slope of the deposition velocities when plotted against $\tau^{+}$is in better agreement with the experimentally observed slope in 
simulations with the optimum lift than in simulations using the unmodified lift (Wang \& Squires, 1996b).

Zhang \& Ahmadi (2000) analyzed particle deposition to vertical and horizontal floor surfaces in a DNS-generated channel flow. This investigation mainly focused on differences in deposition between upward vertical flow and downward vertical flow owing to changes in the lift force; however, the study is unique in that it is the only DNS Lagrangian simulation that includes deposition to a horizontal floor surface. By noting that the initial locations of depositing particles are concentrated in bands, this work also clearly reemphasized that particle deposition to vertical surfaces in the diffusionimpaction regime is associated with high speed down sweeps of fluid periodically spaced in the spanwise direction by about 100 dimensionless units. The same effect was not observed for very small particles where Brownian diffusion is important or for particles depositing to the horizontal floor surface because of the effect of gravity. Deposition velocities from the simulations of Zhang \& Ahmadi are presented in Figures 45-47. Figure 45 demonstrates this effect dramatically in flows with low friction velocities of 3 and $10 \mathrm{~cm} / \mathrm{s}$. In this figure, deposition rates to the vertical wall in the horizontal flow are much lower than those in the vertical downward flow. Furthermore, the increase in deposition in the downward flow is much greater for the smaller friction velocity. This effect can be explained by the fact that gravitational settling leads to a larger slip velocity and, thus, larger lift force in the lower air velocity case. The simulation results from the vertical flows exhibit much higher deposition velocities than the experimental data. At least in part, this difference can be attributed to the experiments being conducted at far greater friction velocities.

Figure 46 shows simulation results comparing vertical upward flow to vertical downward flow at a higher friction velocity of $30 \mathrm{~cm} / \mathrm{s}$. Deposition velocities in these two cases are nearly identical and the flow direction has little impact on the magnitude of the lift force or the deposition velocity at this higher friction velocity. This simulation suggests that the direction of flow is only important for very small friction velocities, less than about $10 \mathrm{~cm} / \mathrm{s}$. This result should be taken with caution considering that the equation used for lift in this simulation has been shown to overpredict the magnitude of the force (Wang et al., 1997).

Simulated deposition rates to the horizontal floor at two friction velocities are compared to the comparable experimental data of Sehmel (1973) in Figure 47. The primary 
deposition mechanism in this case is gravitational settling and excellent agreement between the simulation and the data is observed. As observed in experiments, dimensionless deposition velocities to the horizontal surfaces in these simulations are much higher than those to vertical surfaces and they are highly dependent on the magnitude of the friction velocity.

Lagrangian simulations of particles in turbulent airflows have provided much information regarding particle deposition to surfaces and particle-turbulence interactions. Because individual particle trajectories are calculated in Lagrangian simulations, details of particle motion are accessible that are unattainable by experiment. For particles in the diffusionimpaction regime depositing to vertical walls, temporal deposition patterns and high wallimpact velocities illustrate the importance of near-wall turbulent vortices in influencing particle deposition. Accumulation of particles in the diffusion-impaction regime near vertical walls has also been frequently observed in Lagrangian simulations.

Figure 48 displays deposition velocities from several Lagrangian simulations for particles depositing to smooth vertical surfaces in cases where the lift force was both neglected and included in the particle trajectory calculations. The diffusion, diffusion-impaction and inertia-moderated particle deposition regimes seen in the experimental data in Figure 2 are clearly evident in Figure 48. The deposition data from the Lagrangian simulations are less scattered than the experimentally obtained data, but significant scatter is also present in the simulation results. In the simulations, there are no errors involved in determining particle size or deposition fluxes and this undoubtedly helps to reduce the scatter compared to physical experiments. Changes in factors like particle density, friction velocity (especially at low values of friction velocity) and the method of accounting for forces acting on the particles are most likely to contribute to the spread in the results observed in Figure 48.

Lagrangian simulations of particle deposition to floors have also demonstrated good agreement with experiments; however, no simulations have been performed analyzing deposition to a ceiling. Investigations of particle deposition to rough surfaces by Lagrangian simulations have been limited. The work that has been done suggests similar trends as observed in Eulerian models and experiments in the case of vertical walls. Roughness had a lesser effect in simulations on deposition to a floor. In sum, Lagrangian simulations have proven extremely useful for investigating the behavior of particles in 
turbulent airflows, but their usefulness as tools for predicting particle deposition from a given flow is constrained by high computational cost and time.

\subsection{Empirical Equations for Particle Deposition in Duct Bends}

Most studies of particle deposition in bends have been conducted in laminar flow. Only two models to predict deposition from turbulent flow in bends have been published. Both report empirical equations to calculate particle penetration through a bend. The model of Pui et al. (1987) is a fit to experimental data and the model of McFarland et al. (1997) is a fit to the results of simple Lagrangian simulations.

Pui et al. (1987) measured particle penetration through $90^{\circ}$ bends in $0.503-0.851 \mathrm{~cm}$ diameter tubes and found that the best correlation to their experimental data was given by

$$
P_{\text {bend }}=\frac{C_{\text {outlet }}}{C_{\text {inlet }}}=10^{-0.963 \mathrm{St}}
$$

Support for the form of this equation was provided by arguments from turbulent mixing theory.

McFarland et al. (1997) performed physical experiments and Lagrangian simulations to investigate particle penetration through $45-180^{\circ}$ bends in $1.6 \mathrm{~cm}$ diameter tubes. Only the results of the Lagrangian simulations were used to develop the following empirical equation for predicting particle penetration through bends:

$$
\begin{aligned}
& \ln P_{\text {bend }}=\frac{4.61+a \theta \mathrm{St}}{1+b \theta \mathrm{St}+c \theta \mathrm{St}^{2}+d \theta^{2} \mathrm{St}} \\
& a=-0.9526-0.05686 R_{o} \\
& b=\frac{-0.297-0.0174 R_{o}}{1-0.07 R_{o}+0.0171 R_{o}^{2}} \\
& c=-0.306+\frac{1.895}{\sqrt{R_{o}}}-\frac{2.0}{R_{o}} \\
& d=\frac{0.131-0.0132 R_{o}+0.000383 R_{o}^{2}}{1-0.129 R_{o}+0.0136 R_{o}^{2}}
\end{aligned}
$$


Here, $\theta$ is the bend angle in radians and $R_{o}$ is the bend ratio as defined in equation (18). Note also that the equation for parameter $d$ presented in McFarland et al. has a minor error that is corrected in equation (126). Because the model proposed by McFarland et $a l$. is able to account for a variety of bend angles and bend ratios, it is somewhat more flexible than the equation of Pui et al. which was validated only for $90^{\circ}$ bends with a single bend radius of 5.7. Neither of these bend-penetration models account for changes in the bend orientation with respect to the direction of gravitational acceleration.

A comparison of the two bend-penetration models is given in Figure 49. The models are applied to the case of a $90^{\circ}$ bend in a $15-\mathrm{cm}$ diameter duct with an average air speed of 5 $\mathrm{m} / \mathrm{s}$. Both models predict nearly complete penetration of the smallest particles and nearly complete deposition in the bend for the largest particles. For intermediate sized particles, the model of Pui et al. predicts lower values of particle penetration than the model of McFarland et al. There are no experimental data to evaluate these models when the bends have diameters similar to those in HVAC systems.

Duct bends in HVAC systems may be oriented in a variety of ways and these different orientations are likely to influence the deposition of some particles within the bends. For example, owing to the influence of gravity, a bend that directs a horizontal flow vertically upwards will likely have different particle deposition characteristics than a bend that directs a horizontal flow to flow vertically downwards. Such effects have not been systematically investigated by theory or experiment. We are also unaware of any reports investigating the influence of surface roughness on particle deposition within bends.

\subsection{Summary of Methods for Predicting Particle Deposition Rates}

Airflows through ventilation ducts of commercial buildings are turbulent and span a range of air velocities and flow types. Ventilation ducts vary widely in terms of material, size, cleanliness and internal roughness. Models to predict particle deposition in ventilation ducts should be applicable to the broad range of flow conditions and surface characteristics in HVAC systems and should be able to account for variations in particle size and density.

A variety of techniques for predicting particle deposition from turbulent flows have been presented, ranging in complexity from empirical equations to DNS-based Lagrangian simulations. Empirical equations are based on fits to experimental data using equation 
forms that may or may not have a theoretical foundation. Theoretically based models and simulations have generally been found to underpredict deposition rates compared to experimental measurements.

Empirical equations are simple to use and show good agreement with data from physical experiments and Lagrangian simulations. The main drawbacks of empirical equations are that they offer little insight into particle deposition mechanisms and that their applicability to flows different from those for which they were developed is uncertain. In addition, it is not possible to account for surface roughness in most cases when using empirical equations. Despite these limitations, empirical equations can provide quick estimates of particle deposition velocities, even in cases where thermal gradients or electric fields exist. Among the methods, equations (2.52)-(2.54) along with equations (2.71)-(2.74) are likely to yield reasonable predictions for particle deposition to smooth vertical and horizontal surfaces across all deposition regimes.

Eulerian models include gradient diffusion models, free-flight models and turbophoretic models. Gradient diffusion models have been determined to be inappropriate for the high air velocities and friction velocities typical in HVAC ducts. Experiments and Lagrangian simulations suggest that mechanisms other than Brownian and turbulent diffusion are responsible for the deposition of particles outside of the diffusion regime and these other mechanisms are not taken into account in gradient diffusion models. Free-flight models allow for a free-flight deposition mechanism similar to what is observed in Lagrangian simulations. However, the method of accounting for free flight is crude and free-flight models are based on somewhat unsatisfying and sometimes contradictory assumptions. Most free-flight models rely on empiricism to achieve agreement with experimental data, and those that do not (e.g., Davies, 1966b) substantially underpredict most experimental data. The model of El-Shobokshy \& Ismail (1980) is the recommended free-flight model for application in HVAC ducts. This model is highly empirical, but it achieves good agreement with experiments and simulations for both smooth and rough vertical surfaces. The turbophoretic models of Guha (1997) and Young \& Leeming (1997) are the most appropriate Eulerian models for predicting particle deposition in HVAC ducts. These models are more difficult to apply than other Eulerian models, but they are based on more physically sound assumptions and are the only Eulerian models with the ability to give an accurate accounting of all particle forces, including the turbophoretic force. These turbophoretic models predict deposition rates similar to those observed in experiments and simulations and they predict the accumulation of particles in the diffusion-impaction 
regime near vertical walls, as is observed in Lagrangian simulations. These models exhibit good performance when applied to both vertical and horizontal surfaces and to both smooth and rough surfaces.

Sublayer models simulate near-wall turbulence as a two-dimensional stagnation-point flow and use a Lagrangian scheme in this near-wall layer to estimate deposition rates. The modeled flow is meant to represent the near-wall eddies observed to be responsible for depositing certain particles to vertical surfaces in Lagrangian simulations. These models are not fully Lagrangian and the resulting equations can be solved quickly $(\sim \mathrm{a}$ few seconds) with current computing power. The sublayer model of Fan \& Ahmadi (1993) is the most well-developed in the literature and the best sublayer model for application to HVAC ducts. This sublayer model had been extended to account for surface roughness, electrical charge and nonspherical particles.

Fully Lagrangian simulations have yielded much information that is applicable to particle deposition in HVAC ducts, but such simulations are impractical as predictive tools owing to their current high computational cost. Lagrangian particle simulations with flow fields generated by DNS are currently limited to flow Reynolds numbers less than about 10,000. Flows generated by LES have allowed particle simulations in flows with Reynolds numbers approaching 80,000. These techniques reach into the lower end of the range of flow Reynolds numbers of interest for HVAC ducts, i.e. $10^{4}$ to $3 \times 10^{6}$. The information generated by these simulations can be viewed as the results of numerical experiments, analogous to physical experiments. Deposition velocities from most Lagrangian simulations are within the range observed in physical experiments. Provided that these simulations accurately account for all forces acting on particles near a wall, they can be an excellent source of information for comparison to Eulerian models. As computational power increases, Lagrangian simulations will be able to investigate higher flow Reynolds numbers and more complex flow geometries. But for the near future, these techniques should be viewed as high-end research tools, rather than tools for engineering analysis of real HVAC systems.

Even the most sophisticated particle deposition models have only limited direct applicability to the case of particle deposition in HVAC systems. Except for those few efforts that have dealt explicitly with bends, all equations, models and simulations discussed in this review are concerned exclusively with fully developed turbulent flow in a straight duct. Such flows occur in real ventilation ducts, but the frequent occurrence of 
bends, junctions and obstructions makes developing turbulence a common aspect of HVAC duct flow. No models or simulations have been advanced to help understand particle deposition from turbulent flows with undeveloped velocity profiles.

Roughness elements on the interior of duct surfaces are likely to vary in terms of shape, height and surface density. Surface roughness is typically accounted for in models by changing the criteria for particle deposition by using the method proposed by Browne (1974). This model adjustment was developed specifically for the case of closely packed sand-grain type roughness and considers only the average height of the roughness elements. Most models that account for surface roughness exhibit a similar response, probably because the method of accounting for roughness is fundamentally the same. No models consider other geometrical aspects of roughness elements like width or orientation, and roughness element densities other than closely packed sand-grain type have not been thoroughly addressed.

In summary, predicting particle behavior in turbulent flows with developing velocity profiles and accounting for different types of roughness elements are the main difficulties when modeling particle deposition from flows in ventilation ducts. Further research addressed toward improving these aspects of models, augmented by well-designed laboratory experiments will improve our ability to model particle deposition from turbulent flow in HVAC systems. 


\section{References}

Adam, N., Everitt, P. and Riffat, S.B. (1996) Aerosol deposition in ventilation ducts. International Journal of Energy Research, 20: 1095-1101.

Alexander, L.G. and Coldren, C.L. (1951) Droplet transfer from suspending air to duct walls. Industrial and Engineering Chemistry, 43: 1325-1331.

Altshul, A.D. (1970) Hydraulic Resistance. Moscow: Nedra Press, as cited in Idelchik (1986).

ASHRAE (1989a) ASHRAE Guideline 1-1989, Guideline for commissioning of HVAC systems. Atlanta: American Society of Heating, Refrigeration and Air-Conditioning Engineers.

ASHRAE (1989b) ANSI/ASHRAE Standard 62-1989, Ventilation for acceptable indoor air. Atlanta: American Society of Heating, Refrigeration and Air-Conditioning Engineers.

ASHRAE (1992) ANSI/ASHRAE Standard 55-1992, Thermal environmental conditions for human occupancy. Atlanta: American Society of Heating, Refrigeration and AirConditioning Engineers.

ASHRAE (1995) ASHRAE Handbook: HVAC Applications. Atlanta: American Society of Heating, Refrigeration and Air-Conditioning Engineers.

Batterman, S.A. and Burge, H. (1995) HVAC systems as emission sources affecting indoor air quality: A critical review. $H V A C \& R$ Research, 1: 61-80.

Beal, S.K. (1970) Deposition of particles in turbulent flow on channel or pipe walls. Nuclear Science and Engineering, 40: 1-11.

Bennet, C.O. and Meyers, J.E. (1962) Momentum, Heat and Mass Transfer. New York: McGraw-Hill.

Björkroth, M.K. (1999) Cleanliness of a ventilation system, how clean is clean enough? Proceedings of Indoor Air '99, Edinburgh, Scotland, Vol. 3, pp. 80-85.

Boersma, B.J. and Nieuwstadt, F.T.M. (1996) Large-eddy simulation of turbulent flow in a curved pipe. Journal of Fluids Engineering, 118: 248-254.

Brooke, J.W., Kontomaris, K., Hanratty, T.J. and McLaughlin, J.B. (1992) Turbulent deposition and trapping of aerosols at a wall. Physics of Fluids A, 4: 825-834.

Brooke, J.W., Hanratty, T.J. and McLaughlin, J.B. (1994) Free-flight mixing and deposition of aerosols. Physics of Fluids A, 6: 3404-3415. 
Browne, L.W.B. (1974) Deposition of particles on rough surfaces during turbulent gasflow in a pipe. Atmospheric Environment, 8: 801-816.

Cantwell, B.J. (1981) Organized motion in turbulent flow. Annual Review of Fluid Mechanics, 13: 457-515.

Caporaloni, M., Tampieri, F., Trombetti, F. and Vittori, O. (1975) Transfer of particles in nonisotropic air turbulence. Journal of the Atmospheric Sciences, 32: 565-568.

Cebeci, T. and Smith, A.M.O. (1974) Analysis of Turbulent Boundary Layers. New York: Academic Press.

Chamberlain, A.C. (1967) Transport of lycopodium spores and other small particles to rough surfaces. Proceedings of the Royal Society of London Series A, 296: 45-70.

Chamberlain, A.C., Garland, J.A. and Wells, A.C. (1984) Transport of gases and particles to surfaces with widely spaced roughness elements. Boundary Layer Meteorology, 29: 343-360.

Chapman, D.R. and Kuhn, G.D. (1986) The limiting behavior of turbulence near a wall. Journal of Fluid Mechanics, 170: 265-292.

Chen, M. and McLaughlin, J.B. (1995) A new correlation for the aerosol deposition rate in vertical ducts. Journal of Colloid and Interface Science, 169: 437-455.

Chen, Q. and Ahmadi, G. (1997) Deposition of particles in a turbulent pipe flow. Journal of Aerosol Science, 28: 789-796.

Cheong, K.W. (1997) Deposition of aerosol particles in ductwork. Applied Energy, 57: 253-261.

Cherukat, P. and McLaughlin, J.B. (1994) The inertial lift on a rigid sphere in a linear shear flow field near a flat wall. Journal of Fluid Mechanics, 263: 1-18.

Choi, H., Moin, P. and Kim, J. (1993) Direct numerical simulation of turbulent flow over riblets. Journal of Fluid Mechanics, 255: 503-539.

Cleaver, J.W. and Yates, B. (1975) A sub layer model for the deposition of particles from a turbulent flow. Chemical Engineering Science, 30: 983-992.

Colebrook, F. (1939) Turbulent flow in pipes with particular reference to the transition region between the smooth and rough pipe laws. Journal of the Institution of Civil Engineering, 4: 14-25, as cited in Idelchik (1986). 
Collet, C.W., Nathanson, T., Scott, J.A., Baer, K. and Waddington, J. (1999) The impact of HVAC system cleaning on levels of surface dust and viable fungi in ductwork. Proceedings of Indoor Air '99, Edinburgh, Scotland, Vol. 3, pp. 56-61.

Corino, E.R. and Brodkey, R.S. (1969) A visual investigation of the wall region in turbulent flow. Journal of Fluid Mechanics, 37: 1-30.

Crandall, M.S. and Sieber, W.K. (1996) The National Institute for Occupational Safety and Health indoor environmental evaluation experience, Part I: Building environmental evaluations. Applied Occupational and Environmental Hygiene, 11: 533-539.

Davies C.N. (1965) The rate of deposition of aerosol particles from turbulent flow through ducts. Annals of Occupational Hygiene, 8: 239-245.

Davies, C.N. (1966a) Deposition of aerosols from turbulent flow through pipes. Proceedings of the Royal Society of London Series A, 289: 235-246.

Davies, C.N. (1966b) Brownian deposition of aerosol particles from turbulent flow through pipes. Proceedings of the Royal Society of London Series A, 290: 557-562.

Davies, J.T. (1983) A new theory of aerosol deposition from turbulent fluids. Chemical Engineering Science, 38: 135-139.

Delp, W.W., Matson, N.E., Tschudy, E., Modera, M.P. and Diamond, R.C. (1997) Field investigation of duct system performance in California light commercial buildings. Report LBNL-40102, Berkeley, California: Lawrence Berkeley National Laboratory.

Demare, S., Labraga, L. and Tournier, C. (1999) Comparison and scaling of the bursting period in rough and smooth walls channel flows. Journal of Fluids Engineering, 121: $735-746$.

Durst, F., Milojevic, D. and Schönung, B. (1984) Eulerian and Lagrangian predictions of particulate two-phase flows: a numerical study. Applied Mathematical Modeling, 8: 101115.

Durst, F., Jovanović, J. and Sender, J. (1995) LDA measurements in the near-wall region of a turbulent pipe flow. Journal of Fluid Mechanics, 295: 305-335.

El-Shobokshy, M.S. and Ismail, I.A. (1980) Deposition of aerosol particles from turbulent flow onto rough pipe wall. Atmospheric Environment, 14: 297-304.

El-Shobokshy, M.S. (1983) Experimental measurements of aerosol deposition to smooth and rough surfaces. Atmospheric Environment, 17: 639-644.

Erhart, D.L. (1986) Turbulent aerosol deposition and reentrainment. Ph.D. Dissertation, University of California, Berkeley, California. 
Fan, F-G. and Ahmadi, G. (1993) A sublayer model for turbulent deposition of particles in vertical ducts with smooth and rough surfaces. Journal of Aerosol Science, 24: 45-64.

Fan, F-G. and Ahmadi, G. (1994) On the sublayer model for turbulent deposition of aerosol particles in the presence of gravity and electric fields. Aerosol Science and Technology, 21: 49-71.

Fan, F-G. and Ahmadi, G. (1995) A sublayer model for wall deposition of ellipsoidal particles in turbulent streams. Journal of Aerosol Science, 26: 813-840.

Fichman, M., Gutfinger, C. and Pnueli, D. (1988) A model for turbulent deposition of aerosols. Journal of Aerosol Science, 19: 123-136.

Fisk, W.J., Delp, W., Diamond, R., Dickerhoff, D., Levinson, R., Modera, M., Nematollahi, M. and Wang, D. (1999) Duct systems in large commercial buildings: Physical characterization, air leakage and heat conduction gains. Report LBNL-42339, Berkeley, California: Lawrence Berkeley National Laboratory.

Forney, L.J. and Spielman, L.A. (1974) Deposition of coarse aerosols from turbulent flow. Aerosol Science, 5: 257-271.

Fransson, J.I. (1996) Particle deposition in ventilation air supply ducts. Proceedings of Indoor Air '96, Nagoya, Japan, Vol. 2, pp. 717-722.

Friedlander, S.K. and Johnstone, H.F. (1957) Deposition of suspended particles from turbulent gas streams. Industrial and Engineering Chemistry, 49: 1151-1156.

Friedlander, S.K. (1977) Smoke, Dust and Haze: Fundamentals of Aerosol Behavior. New York: John Wiley \& Sons.

Friedrich, R., Hüttl, T.J., Manhart, M. and Wagner, C. (2001) Direct numerical simulation of incompressible turbulent flows. Computers and Fluids, 30: 555-579.

Gavrilakis, S. (1992) Numerical simulation of low-Reynolds-number turbulent flow through a straight square duct. Journal of Fluid Mechanics, 244: 101-129.

Gieseke, J.A., Lee, K.W. and Goldenberg, M.A. (1980) Measurement of aerosol deposition rate in turbulent flows. NUREG/CR-1262, BMI-2041, as cited in Erhart (1986).

Gouesbet, G. and Berlemont, A. (1999) Eulerian and Lagrangian approaches for predicting the behavior of discrete particles in turbulent flows. Progress in Energy and Combustion Science, 25: 133-159. 
Grass, A.J. (1971) Structural features of turbulent flow over smooth and rough boundaries, Journal of Fluid Mechanics, 50: 238-255.

Grass, A.J., Stuart, R.J. and Mansour-Tehrani, M. (1993) Common vortical structure of turbulent flows over smooth and rough boundaries. AIAA Journal, 31: 837-847.

Guha, A. (1997) A unified Eulerian theory of turbulent deposition to smooth and rough surfaces. Journal of Aerosol Science, 28: 1517-1537.

Hahn L.A., Stukel, J.J., Leong, K.H. and Hopke, P.K. (1985) Turbulent deposition of submicron particles on rough walls. Journal of Aerosol Science, 16: 81-86.

Hanley, J.T., Ensor, D.S., Smith, D.D. and Sparks, L.E. (1994) Fractional aerosol filtration efficiency of in-duct ventilation air cleaners. Indoor Air, 4: 169-178.

He, C. and Ahmadi, G. (1998) Particle deposition with thermophoresis in laminar and turbulent duct flows. Aerosol Science and Technology, 29: 525-546.

Hinze, J.O. (1975) Turbulence, $2^{\text {nd }}$ Edition. New York: McGraw-Hill.

Holopainen, R., Palonen, J. and Seppänen, O. (1999) Measuring methods for determining the level of dust in the air duct. Proceedings of Indoor Air '99, Edinburgh, Scotland, Vol. 3, pp. 103-108.

Huser, A. and Biringen, S. (1993) Direct numerical simulation of turbulent flow in a square duct. Journal of Fluid Mechanics, 257: 65-95.

Hutchinson P., Hewitt, G.F. and Dukler, A.E. (1971) Distribution of liquid or solid dispersions from turbulent gas streams: a stochastic model. Chemical Engineering Science, 26: 419-439.

Idelchik, I.E. (1986) Handbook of Hydraulic Resistance, $2^{\text {nd }}$ Edition. New York: Hemisphere Publishing.

Ilori, T.A. (1971) Turbulent deposition of aerosol particles inside pipes. Ph.D. Dissertation, University of Minnesota, Minneapolis, Minnesota.

Im, K.H and Ahluwalia, R.K. (1989) Turbulent eddy deposition of particles on smooth and rough surfaces. Journal of Aerosol Science, 20: 431-436.

Ishikawa, K., Iwata, T., Kumagai, K., Kimura, K. and Yoshizawa, S. (1999) Field investigation on the effects of duct cleaning on indoor air quality with measured results of TVOC and perceived air quality. Proceedings of Indoor Air '96, Nagoya, Japan, Vol. 2, pp. 809-814. 
Johansen, S.T. (1991) The deposition of particles on vertical walls. International Journal of Multiphase Flow, 17: 355-376.

Kallio, G.A. and Reeks, M.W. (1989) A numerical simulation of particle deposition in turbulent boundary layers. International Journal of Multiphase Flow, 15: 433-446.

Kay, J.M. and Nedderman, R.M. (1990) Fluid Mechanics and Transfer Processes, New York: Cambridge University Press.

Kim, J., Moin, P. and Moser, R. (1987) Turbulence statistics in fully developed channel flow at low Reynolds number. Journal of Fluid Mechanics, 177: 133-166.

Kline, S.J., Reynolds, W.C., Schraub, F.A. and Runstadler, P.W. (1967) The structure of turbulent boundary layers. Journal of Fluid Mechanics, 30: 741-773.

Kneen, T. and Strauss, W. (1969) Deposition of dust from turbulent gas streams. Atmospheric Environment, 3: 55-67.

Krogstad, P-Å., Antonia, R.A. and Browne, L.W.B. (1992) Comparison between roughand smooth-wall turbulent boundary layers. Journal of Fluid Mechanics, 245: 599-617.

Krogstad, P-Å. and Antonia, R.A. (1999) Surface roughness effects in turbulent boundary layers. Experiments in Fluids, 27: 450-460.

Kvasnak, W., Ahmadi, G., Bayer, R. and Gaynes, M. (1993) Experimental investigation of dust particle deposition in a turbulent channel flow. Journal of Aerosol Science, 24: 795-815.

Kvasnak, W. and Ahmadi, G. (1996) Deposition of ellipsoidal particles in turbulent duct flows. Chemical Engineering Science, 51: 5137-5148.

Laatikainen, T., Pasanen, P., Korhonen, L., Nevalainen, A. and Ruuskanen, J. (1991) Methods for evaluating dust accumulation in ventilation ducts. Proceedings of Healthy Buildings 1991, Washington D.C., pp. 379-382.

Labraga, L., Mazouz, A., Demare, S. and Tournier, C. (1997) Effect of roughness aspect ratio on the "bursting" period in a fully turbulent channel flow. Journal of Fluids Engineering, 119: 469-471.

Lai, C.K. (1997) An experimental study of the deposition of aerosol on rough surfaces and the implications for indoor air quality control. Ph.D. Dissertation, Imperial College, London, England.

Lai, A.C.K. and Nazaroff, W.W. (2000) Modeling indoor particle deposition from turbulent flow onto smooth surfaces. Journal of Aerosol Science, 31: 463-476. 
Laufer, J. (1954) The structure of turbulence in fully developed pipe flow. NACA Technical Note 1174, pp. 417-434.

Lee, K.W. and Gieseke, J.A. (1994) Deposition of particles in turbulent pipe flows. Journal of Aerosol Science, 25: 699-709.

Levinson, R., Delp, W., Dickerhoff, D., Fisk, W., Nematollahi, M., Stordahl, I., Torre, C., Wang, D., Diamond, R. and Modera, M. (1997) Commercial thermal distribution systems: Final report for California Institute for Energy Efficiency. Report LBNL-41045, Berkeley, California: Lawrence Berkeley National Laboratory.

Li, A. and Ahmadi, G. (1993a) Deposition of aerosols on surfaces in a turbulent channel flow. International Journal of Engineering Science, 31: 435-451.

Li, A. and Ahmadi, G. (1993b) Computer simulation of deposition of aerosols in a turbulent channel flow with rough walls. Aerosol Science and Technology, 18: 11-24.

Li, A. and Ahmadi, G. (1993c) Aerosol particle deposition with electrostatic attraction in a turbulent channel flow. Journal of Colloid and Interface Science, 158: 476-482.

Li, A., Ahmadi, G., Bayer, R.G. and Gaynes, M.A. (1994) Aerosol particle deposition in an obstructed turbulent duct flow. Journal of Aerosol Science, 25: 91-112.

Lin, C.S., Moulton, R.W. and Putnam, G.L. (1953) Mass transfer between solid wall and fluid streams: Mechanism and eddy distribution relationships in turbulent flow. Industrial and Engineering Chemistry, 45: 636-640.

Liu, B.Y.H. and Agarwal, J.K. (1974) Experimental observation of aerosol deposition in turbulent flow. Aerosol Science, 5 :145-155.

Liu, B.Y.H. and Ilori, T.A. (1974) Aerosol deposition in turbulent pipe flow. Environmental Science and Technology, 8: 351-356.

Luoma, M., Pasanen, P. and Ikäheimo, M. (1999) The effect of duct cleaning on indoor air quality in office buildings. Proceedings of Indoor Air '99, Edinburgh, Scotland, Vol. 3, pp. 91-96.

Maxey, M.R. and Riley, J.R. (1983) Equation of motion for a small rigid sphere in a nonuniform flow. Physics of Fluids, 26: 883-889.

Mazouz, A., Labraga, L. and Tournier, C. (1998) Anisotropy invariants of Reynolds stress tensor in a duct flow and turbulent boundary layer. Journal of Fluids Engineering, 120: $280-284$.

McCoy, D.D. and Hanratty, T.J. (1977) Rate of deposition of droplets in annular twophase flow. International Journal of Multiphase Flow, 3: 319-331. 
McFarland, A.R., Gong, H., Muyshondt, A., Wente, W.B. and Anand, N.K. (1997) Aerosol deposition in bends with turbulent flow. Environmental Science and Technology, 31: 3371-3377.

McLaughlin, J.B. (1989) Aerosol particle deposition in numerically simulated channel flow. Physics of Fluids A, 1: 1211-1224.

McLaughlin, J.B. (1991) Inertial migration of a small sphere in linear shear flows. Journal of Fluid Mechanics, 224: 261-274.

McLaughlin, J.B. (1993) The lift on a small sphere in wall-bounded linear shear flows. Journal of Fluid Mechanics, 246: 249-265.

Miyake, Y., Tsujimoto, K. and Agata, Y. (2000) A DNS of a turbulent flow in a roughwall channel using roughness elements model. JSME International Journal Series BFluids and Thermal Engineering, 43: 233-242.

Moin, P. and Mahesh, K. (1998) Direct numerical simulation: A tool in turbulence research. Annual Review of Fluid Mechanics, 30: 539-578.

Montgomery, T.L. and Corn, M. (1970) Aerosol deposition in a pipe with turbulent airflow. Aerosol Science, 1: 185-213.

Morrison, G.C., Nazaroff, W.W., Cano-Ruiz, J.A., Hodgson, A.T. and Modera, M.P. (1998) Indoor air quality impacts of ventilation ducts: Ozone removal and emissions of volatile organic compounds. Journal of the Air and Waste Management Association, 48: 941-952.

Moser, R.D., Kim, J. and Mansour, N.N. (1999) Direct numerical simulation of turbulent channel flow up to $\mathrm{Re}_{\tau}=590$. Physics of Fluids, 11: 943-945.

Muyshondt, A., Anand, N.K. and McFarland, A.R. (1996) Turbulent deposition of aerosol particles in large transport tubes. Aerosol Science and Technology, 24: 107-116.

NADCA (1992) NADCA 1992-01, Mechanical cleaning of non-porous air conveyance system components. Washington, DC: National Air Duct Cleaners Association.

Nikuradse, I. (1936) Laws governing turbulent flow in smooth tubes. In Probl. Turnulentnosti, Edited by Velikanov, M.I. and Shveikovsky, N.G., Moscow: ONTI Press, pp. 75-150, as cited in Idelchik (1986).

Ostro, B. and Chestnut, L. (1998) Assessing the health benefits of reducing particulate matter air pollution in the United States. Environmental Research, 76: 94-106. 
Ottney, T.C. (1993) Particle management for HVAC Systems. ASHRAE Journal, 35 (7): 26-34.

Ounis, H., Ahmadi, G. and McLaughlin, J.B. (1991) Dispersion and deposition of Brownian particles from point sources in a simulated turbulent channel flow. Journal of Colloid and Interface Science, 147: 233-250.

Ounis, H., Ahmadi, G. and McLaughlin, J.B. (1993) Brownian particle deposition in a directly simulated turbulent channel flow. Physics of Fluids A - Fluid Dynamics, 5: 1427-1432.

Owen, P.R. (1960) Dust deposition from a turbulent airstream. International Journal of Air Pollution, 3: 8-25.

Owen, P.R. (1969) Pneumatic transport. Journal of Fluid Mechanics, 39: 407-432.

Papavergos, P.G. and Hedley, A.B. (1984) Particle deposition behaviour from turbulent flows. Chemical Engineering Research and Design, 62: 275-295.

Parker, D.S., Sherwin, J.R., Raustad, R.A. (1997) Impact of evaporator airflow in residential air-conditioning systems. ASHRAE Transactions, 103: 395-405.

Pasanen, P., Nevalainen, A., Ruuskanen, J. and Kalliokoski, P. (1992) The composition and location of dust settled in supply air ducts. Proceedings of the $13^{\text {th }}$ AIVC Conference on Ventilation for Energy Efficiency and Optimum Air Quality, Nice, France, pp. 481488.

Pasanen, P. (1994) Impurities in ventilation ducts. Proceedings of IAQ '94: Engineering Indoor Environments, Atlanta: American Society of Heating, Refrigerating and AirConditioning Engineers, pp. 149-153.

Pasanen, P.O., Pasanen, A-L. and Kalliokoski, P. (1995) Hygienic aspects of processing oil residues in ventilation ducts. Indoor Air, 5: 62-68.

Pasanen, P., Salo, J., Hyttinen, M., Vartiainen, M. and Kalliokoski, P. (2000) Ozone reduction in supply air filters. Proceedings of Healthy Buildings 2000, Helsinki, Finland, Vol. 2, pp. 263-268.

Pearce, D. and Crowards, T. (1996) Particulate matter and human health in the United Kingdom. Energy Policy, 24: 609-619.

Pope, C.A. and Dockery, D.W. (1999) Epidemiology of particle effects. In Air Pollution and Health, Edited by Holgate, S.T., Samet, J.M., Koren, H.S. and Maynard, R.L., London: Academic Press, pp. 673-705. 
Pope, C.A. (2000) Review: Epidemiological basis for particulate air pollution health standards. Aerosol Science and Technology, 32: 4-14.

Postma, A.K. and Schwendiman, L.C. (1960) Studies in micrometrics: I. Particle deposition in conduits as a source of error in aerosol sampling. Report HW-65308, Richland, Washington: Hanford Laboratory.

Pui, D.Y.H., Romay-Novas, F. and Liu, B.Y.H. (1987) Experimental study of particle deposition in bends of circular cross section. Aerosol Science and Technology, 7: 301315.

Reeks, M. W. and Skyrme, G. (1976) The dependence of particle deposition velocity on particle inertia in turbulent pipe flow. Journal of Aerosol Science, 7: 485-495.

Reeks, M. W. (1983) The transport of discrete particles in inhomogeneous turbulence. Journal of Aerosol Science, 14: 729-739.

Rouhiainen, P.O. and Stachiewicz, J.W. (1970) On the deposition of small particles from turbulent streams. Journal of Heat Transfer, 92: 169-177.

Sabot, J., Saleh, I. and Comte-Bellot, G. (1977) Effects of roughness on the intermittent maintenance of Reynolds shear stress in pipe flow. Physics of Fluids, 20: S150-S155.

Saffman, P.G. (1965) The lift on a small sphere in a slow shear flow. Journal of Fluid Mechanics, 22: 385-400.

Saffman, P.G. (1968) Corrigendum to 'The lift on a small sphere in a slow shear flow.' Journal of Fluid Mechanics, 31: 624.

Schlichting, H. (1979) Boundary-Layer Theory, $7^{\text {th }}$ Edition. New York: McGraw-Hill.

Schwartz J., Dockery D.W. and Neas, L.M. (1996) Is daily mortality associated specifically with fine particles? Journal of the Air and Waste Management Association, 46: 927-939.

Sehmel, G.A. (1968) Aerosol deposition from turbulent airstreams in vertical conduits. Report BNWL-578, Richland, Washington: Pacific Northwest Laboratory.

Sehmel, G.A. (1970a) Turbulent deposition of monodisperse particles on simulated grass. In Assessment of Airborne Particles: Fundamentals, Applications, and Implications to Inhalation Toxicity, Edited by Mercer, T.T., Morrow, P.E. and Stöber, W., Springfield, Illinois: Charles C. Thomas, pp. 18-42.

Sehmel, G.A. (1970b) Particle deposition from turbulent air flow. Journal of Geophysical Research, 75: 1766-1781. 
Sehmel, G.A. (1973) Particle eddy diffusivities and deposition velocities for isothermal flow and smooth surfaces. Aerosol Science, 4: 125-138.

Sehmel, G.A. (1980) Particle and gas dry deposition: A review. Atmospheric Environment, 14: 983-1011.

Shaw, D.A. and Hanratty, T.J. (1977) Turbulent mass transfer rates to a wall for large Schmidt numbers. AIChE Journal 23: 28-37.

Shimada, M., Okuyama, K. and Asai, M. (1993) Deposition of submicron aerosol particles in turbulent and transitional flow. AIChE Journal, 39: 17-26.

Siegel, J.A. (2002) Particulate fouling of HVAC heat exchangers. Ph.D. Dissertation, University of California, Berkeley, California.

Stavropoulos, N. (1954) Deposition of particles from turbulent gas streams. M.Sc. Thesis, Columbia University, New York.

Ström, L. (1972) Transmission efficiency of aerosol sampling lines. Atmospheric Environment, 6: 133-142.

Talbot, L., Cheng, R.K., Schefer, R.W. and Willis, D.R. (1980) Thermophoresis of particles in a heated boundary layer. Journal of Fluid Mechanics, 101: 737-758.

Townsend, A.A. (1956) The Structure of Turbulent Shear Flow, Cambridge, England: Cambridge University Press.

Uijttewaal, W.S.J. and Oliemans, R.V.A. (1996) Particle dispersion and deposition in direct numerical and large eddy simulations of vertical pipe flows. Physics of Fluids, $\mathbf{8}$ : 2590-2604.

Valbjørn, O., Nielsen, J.B., Gravesen, S. and Mølhave, L. (1990) Dust in ventilation ducts. Proceedings of Indoor Air '90, Toronto, Canada, Vol. 3, pp.361-364.

Wagner, C. and Friedrich, R. (1998) On the turbulence structure in solid and permeable pipes. International Journal of Heat and Fluid Flow, 19: 459-469.

Wallace, L. (2000) Correlations of personal exposure to particles with outdoor air measurements: A review of recent studies. Aerosol Science and Technology, 32: 15-25.

Wallin, O. (1994) Computer simulation of particle deposition in ventilating duct systems. Ph.D. Dissertation, Royal Institute of Technology, Stockholm, Sweden.

Wang, Q. and Squires, K.D. (1996a) Large eddy simulation of particle-laden turbulent channel flow. Physics of Fluids, 8: 1207-1223. 
Wang, Q. and Squires, K.D. (1996b) Large eddy simulation of particle deposition in a vertical turbulent channel flow. International Journal of Multiphase Flow, 22: 667-683.

Wang, Q., Squires, K.D., Chen, M. and McGlaughlin, J.B. (1997) On the role of the lift force in turbulence simulations of particle deposition. International Journal of Multiphase Flow, 23: 749-763.

Wargocki, P., Wyon, D.P., Sundell, J., Clausen, G. and Fanger, P.O. (2000) The effects of outdoor air supply rate in an office on perceived air quality, Sick Building Syndrome (SBS) symptoms and productivity. Indoor Air, 10: 222-236.

Wasan, D.T. and Wilke, C.R. (1964) Turbulent exchange of momentum, mass and heat between fluid streams and pipe wall. International Journal of Heat and Mass Transfer, 7: 87-94.

Wasan, D.T., Sood, S.K., Davies, R. and Lieberman, A. (1973) Aerosol transport: Particle charge and re-entrainment effects. Journal of Colloid and Interface Science, 43: 144-149.

Wells, A.C. and Chamberlain, A.C. (1967) Transport of small particles to vertical surfaces. British Journal of Applied Physics, 18: 1793-1799.

White, F.M. (1986) Fluid Mechanics, $2^{\text {nd }}$ Edition. New York: McGraw-Hill.

Wood, N.B. (1981a) A simple method for the calculation of turbulent deposition to smooth and rough surfaces. Journal of Aerosol Science, 12: 275-290.

Wood, N.B. (1981b) The mass transfer of particles and acid vapour to cooled surfaces. Journal of the Institute of Energy, 76: 76-93.

Yoder, J.D. and Silverman, L. (1967) Influence of turbulence on aerosol agglomeration and deposition in a pipe. Paper No. 67-33, 60th Annual Air Pollution Control Association Meeting, Cleveland, Ohio.

Young, J. and Leeming, A. (1997) A theory of particle deposition in turbulent pipe flow. Journal of Fluid Mechanics, 340: 129-159.

Yudine, M.I. (1959) Physical considerations on heavy-particle diffusion. Advances in Geophysics, 6: 185-191.

Zhang, H. and Ahmadi, G. (2000) Aerosol particle transport and deposition in vertical and horizontal turbulent duct flows. Journal of Fluid Mechanics, 406: 55-80. 
Table 1. Measured densities of dust on supply duct floors in office buildings and schools.

\begin{tabular}{|c|c|c|c|}
\hline Investigator & $\begin{array}{c}\text { Number } \\
\text { of samples }\end{array}$ & $\begin{array}{c}\text { Mean (range) } \\
\left(\mathrm{g} \mathrm{m}^{-2}\right)\end{array}$ & $\begin{array}{c}\text { Accumulation rate } \\
\left(\mathrm{g} \mathrm{m}^{-2} \mathrm{y}^{-1}\right)\end{array}$ \\
\hline Valbjørn et al. (1990) & NR & $6.8(1.1-51)$ & 0.7 \\
\hline Laatikainen et al. (1991) & 27 & $18(3.6-140)$ & $2.3(0.5-13)$ \\
\hline Pasanen et al. (1992) & 44 & $11(1.2-58)$ & $3.5(1.2-8.3)$ \\
\hline Pasanen (1994) & 44 & $13(1.2-160)$ & 1.0 \\
\hline Fransson (1996) & 15 & $4.0(1.7-12)$ & $0.2-0.3$ \\
\hline Ishikawa et al. (1996) & 6 & $10(2.0-19)$ & NR \\
\hline Björkroth (1999) & 15 & $4.1(0.04-11)$ & NR \\
\hline Collet et al. (1999) & 21 & $12(0.1-59)$ & NR \\
\hline Luoma et al. (1999) & 17 & $6.5(0.7-47)$ & $0.6(0.1-5.9)$ \\
\hline
\end{tabular}

Table 2. Equations for the Fanning friction factor in ducts with rough walls.

\begin{tabular}{|l|l|}
\hline \multicolumn{1}{|c|}{ Reference } & \multicolumn{1}{c|}{ Correlation } \\
\hline Nikuradse $^{\mathrm{a}}(1936)$ & $\frac{1}{\sqrt{f}}=A_{1}+B_{1} \log (\operatorname{Re} \sqrt{f})+C_{1} \log \left(k / D_{h}\right)$ \\
\hline Colebrook (1939) & $\frac{1}{\sqrt{f}}=4.0 \log \left(\frac{2.51}{\operatorname{Re} \sqrt{f}}+\frac{k}{3.7 D_{h}}\right)$ \\
\hline $\begin{array}{l}\text { Bennet \& Meyers } \\
\text { (1962) }\end{array}$ & $\frac{1}{\sqrt{f}}=2.14-4.06 \log \left(\frac{4.73}{\operatorname{Re} \sqrt{f}}+\frac{k}{D_{h}}\right)$ \\
\hline Altshul (1970) & $f=0.0275\left(\frac{68}{\operatorname{Re}}+\frac{k}{D_{h}}\right)^{0.25}\left[\frac{6.9}{\operatorname{Re}}+\left(\frac{k}{3.7 D_{h}}\right)^{1.11}\right]$ \\
\hline White (1986) & $\frac{1}{\sqrt{f}}=-3.6 \log \left[\frac{1}{2}\right.$ \\
\hline
\end{tabular}

${ }^{a}$ As reported in Ch. 2 of Idelchik (1986), the constants $A_{l}, B_{l} \& C_{l}$ depend on the value of the quantity $\operatorname{Re} k \sqrt{f} / D_{h}$ as follows:

\begin{tabular}{|c|c|c|c|}
\hline Value of $\operatorname{Re} k \sqrt{f} / D_{h}$ & $\boldsymbol{A}_{\boldsymbol{I}}$ & $\boldsymbol{B}_{\boldsymbol{1}}$ & $\boldsymbol{C}_{\boldsymbol{I}}$ \\
\hline $3.6-10$ & -0.8 & 2.0 & 0 \\
\hline $10-20$ & 0.068 & 1.13 & -0.87 \\
\hline $20-40$ & 1.538 & 0 & -2.0 \\
\hline $40-191.2$ & 2.471 & -0.588 & -2.588 \\
\hline$>191.2$ & 1.138 & 0 & -2.0 \\
\hline
\end{tabular}




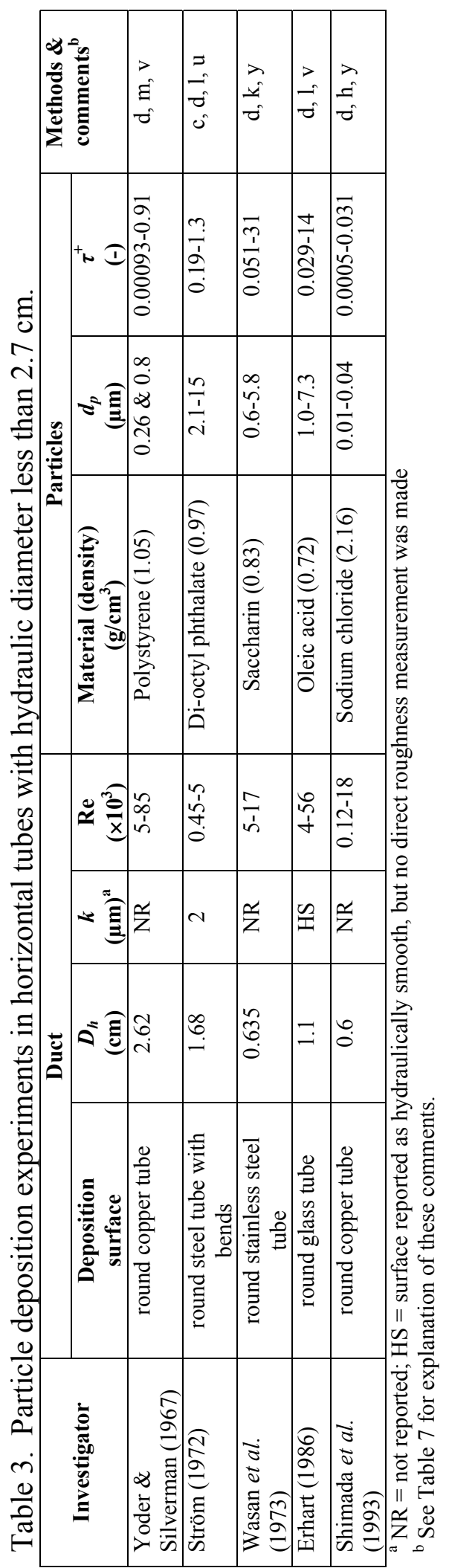




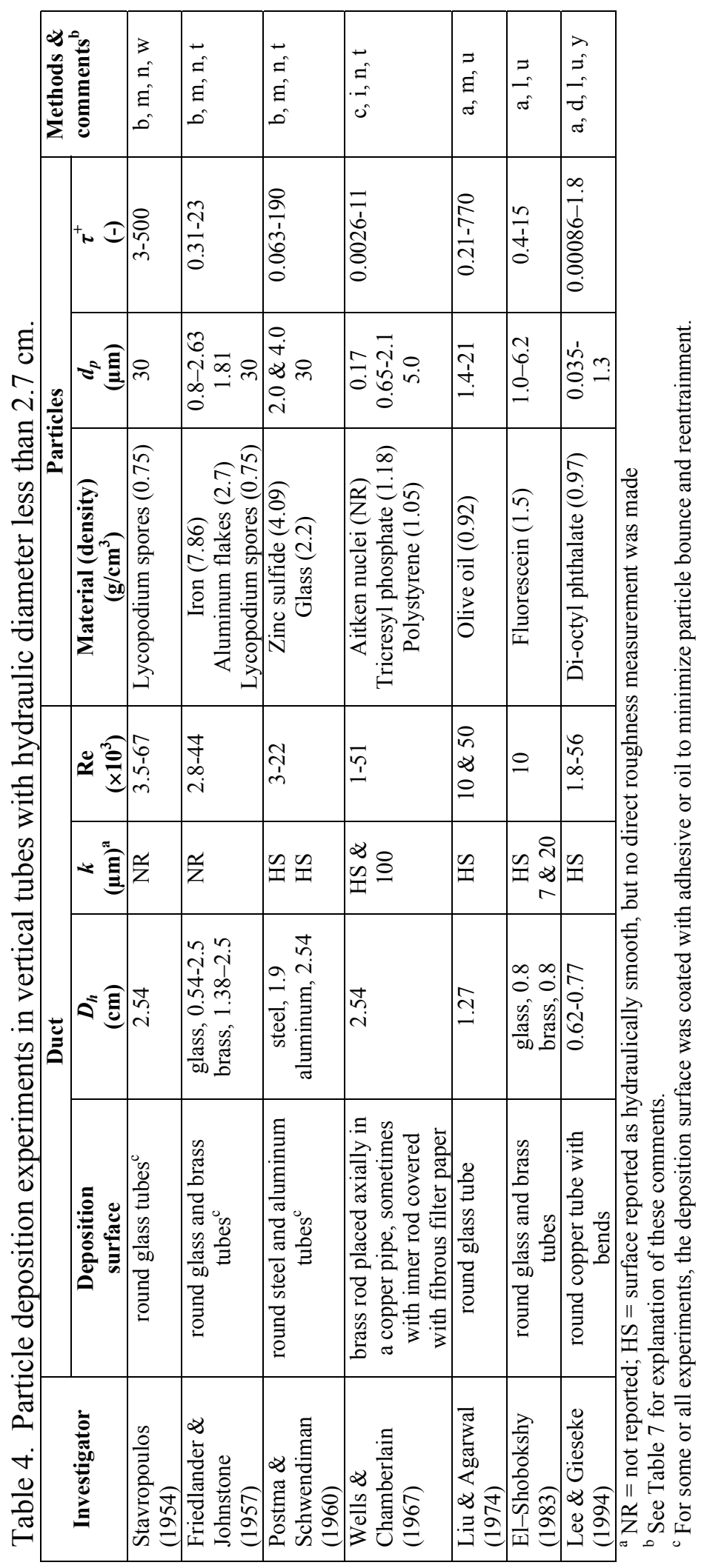




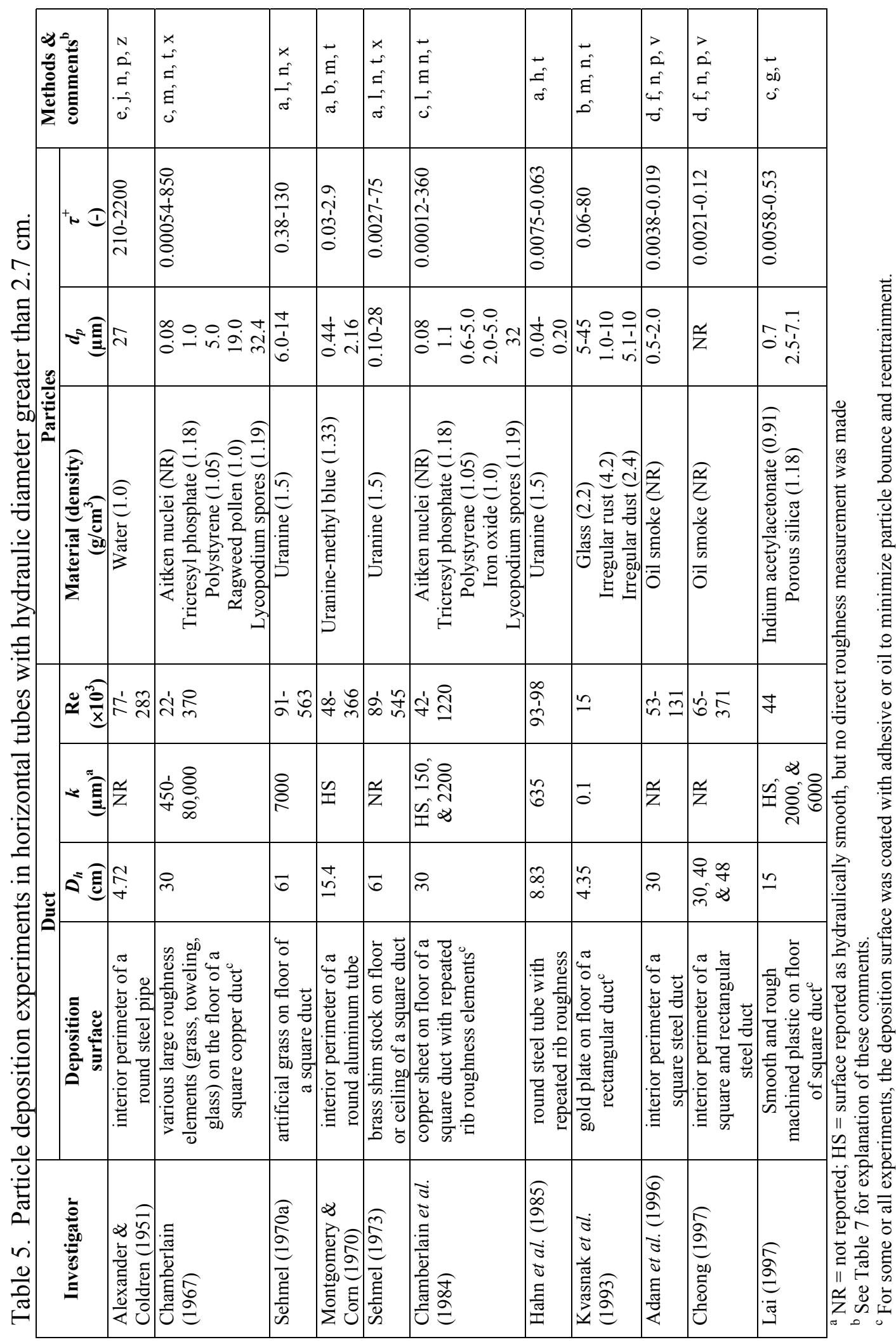




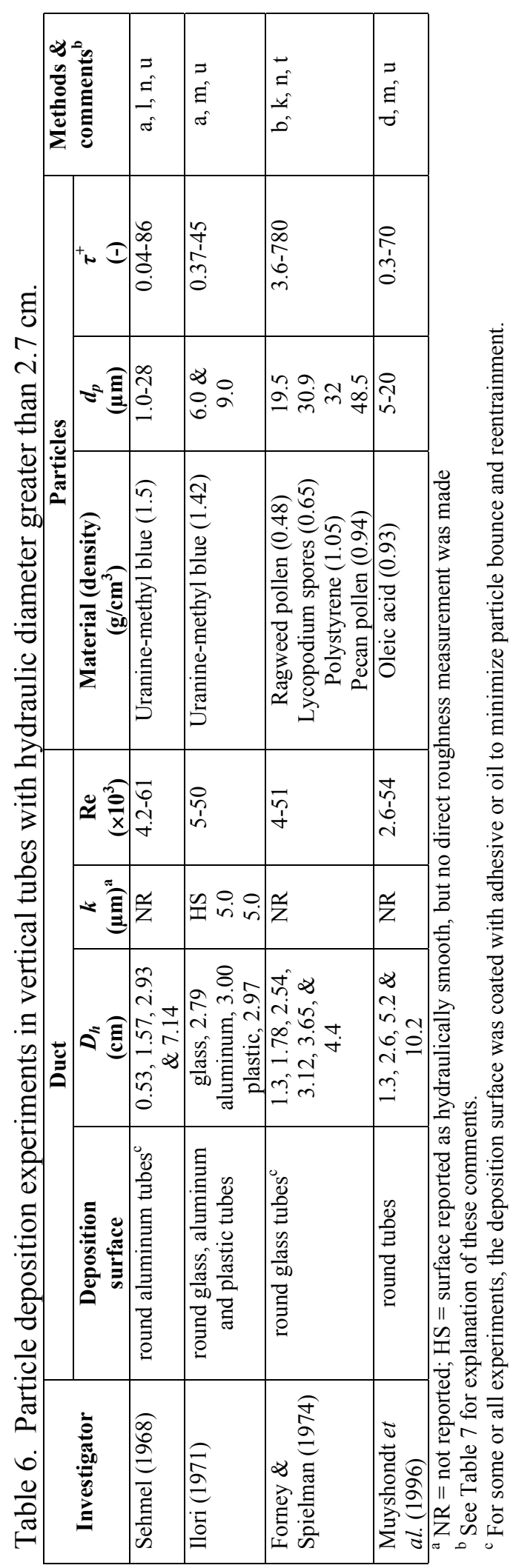




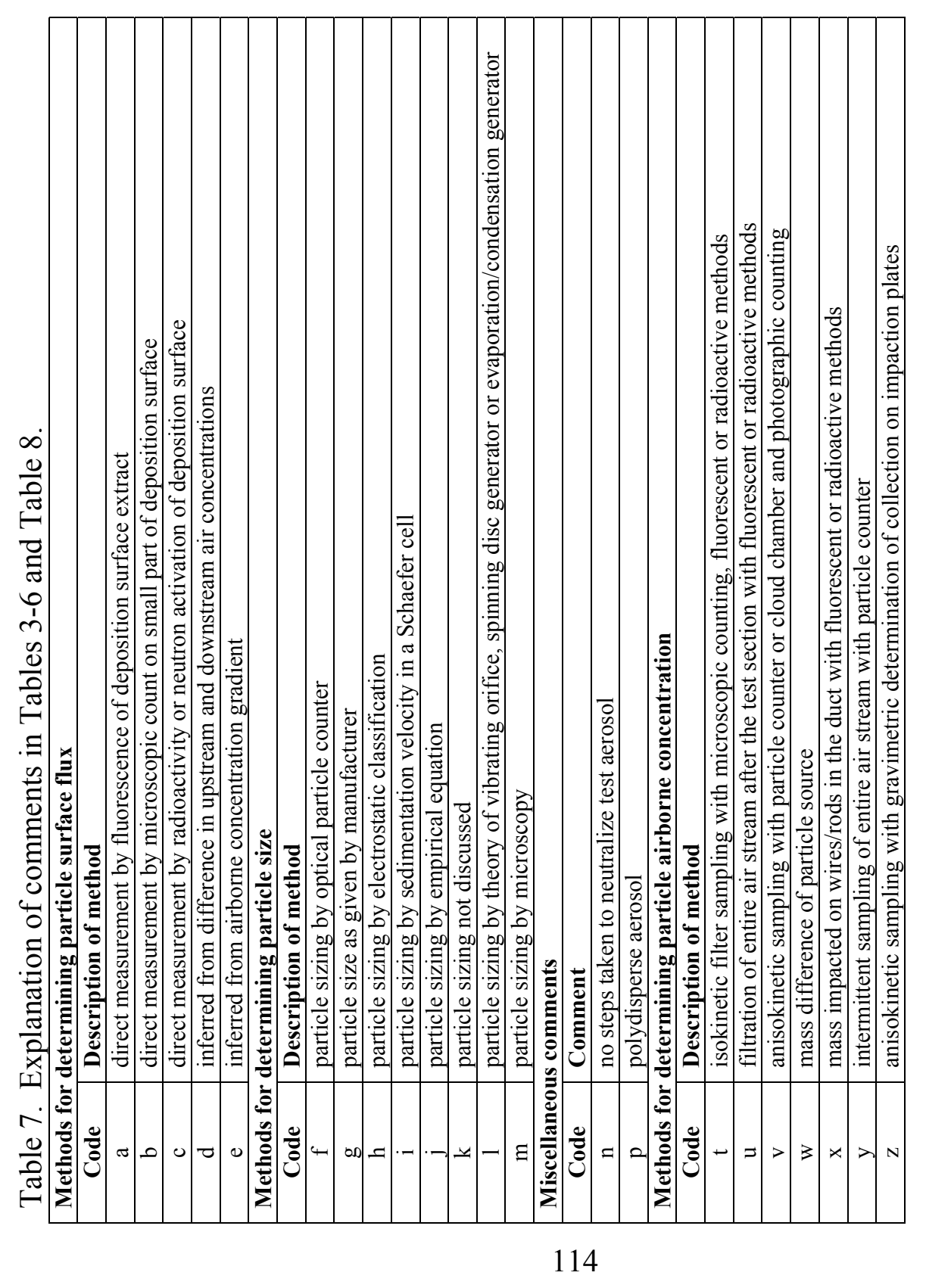




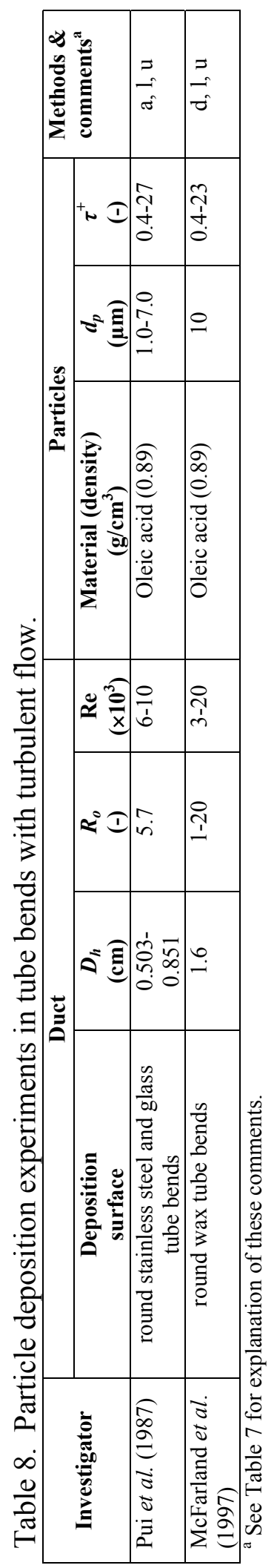


Table 9. Correlations for the eddy viscosity of air.

\begin{tabular}{|c|c|c|}
\hline Investigator & Correlation for the eddy viscosity of air, $\xi_{a}$ & Comments \\
\hline Lin et al. (1953) & $\begin{array}{rr}\frac{\xi_{a}}{v}=\left(\frac{y^{+}}{14.5}\right)^{3} & y^{+} \leq \\
\frac{\xi_{a}}{v}=\frac{y^{+}}{5}-0.959 & 5<y^{+} \leq\end{array}$ & $\begin{array}{l}\text { Best fit to previously } \\
\text { measured velocity } \\
\text { distributions. } \\
\text { Discontinuous at } y^{+}=5 \text {. }\end{array}$ \\
\hline Owen (1960) & $\begin{array}{l}\frac{\xi_{a}}{v}=\left(\frac{y^{+}}{10}\right)^{3} \\
\frac{\xi_{a}}{v}=0.012\left(y^{+}-1.6\right)^{2} \\
\frac{\xi_{a}}{v}=0.4\left(y^{+}-10\right)\end{array}$ & $\begin{array}{l}\text { Best fit to previously } \\
\text { measured velocity } \\
\text { distributions. } \\
\text { Smoother correlation than } \\
\text { that of Lin et al. (1953). }\end{array}$ \\
\hline $\begin{array}{l}\text { Wasan \& Wilke } \\
\text { (1964) }\end{array}$ & $\frac{\xi_{a}}{v}=\frac{4.16 \times 10^{-4} y^{+^{3}}-1.515 \times 10^{-5} y^{+^{4}}}{1-4.16 \times 10^{-4} y^{+^{3}}+1.515 \times 10^{-5} y^{4^{4}}} \quad y^{+} \leq 20$ & $\begin{array}{l}\text { Expression based on } \\
\text { empirical logarithmic } \\
\text { velocity distribution }\end{array}$ \\
\hline Davies (1966a) & $\frac{\xi_{a}}{v}=\frac{\left.y^{+^{\left(4-y^{+}\right.}}\right)}{10^{3}\left(\frac{2.5 \times 10^{7}}{\operatorname{Re}}\right)\left(\frac{y^{+}}{y^{+}+400}\right)} \quad 0.05$ & $\begin{array}{l}\text { Only correlation with a } \\
\text { dependence on Re. } \\
\xi_{a} \text { is not proportional to } \\
y^{+3} \text { as the wall is } \\
\text { approached. }\end{array}$ \\
\hline $\begin{array}{l}\text { Cebeci \& Smith } \\
\text { (1974) }\end{array}$ & $\frac{\xi_{a}}{v}=0.41 y^{+}\left[1-\exp \left(\frac{y^{+}}{26.0}\right)\right]^{2}$ & No comment. \\
\hline $\begin{array}{l}\text { Lai \& Nazaroff } \\
(2000)\end{array}$ & $\begin{array}{l}\frac{\xi_{a}}{v}=7.669 \times 10^{-4} y^{+^{3}} \\
\frac{\xi_{a}}{v}=0.001 y^{+^{2.8214}} \\
\frac{\xi_{a}}{v}=0.0107 y^{1.8895}\end{array}$ & $\begin{array}{l}\text { Best fit to velocity } \\
\text { statistics from DNS of } \\
\text { channel flow by Kim et } \\
\text { al. (1987). }\end{array}$ \\
\hline
\end{tabular}


Table 10. Recommended values of $k_{l}$ for equation (50).

\begin{tabular}{|l|c|}
\hline \multicolumn{1}{|c|}{ Investigator } & $\boldsymbol{k}_{\boldsymbol{1}}$ \\
\hline Cleaver \& Yates (1975) & 0.084 \\
\hline Friedlander (1977) & 0.059 \\
\hline Wood (1981b) & 0.045 \\
\hline Davies (1983) & 0.075 \\
\hline Papavergos \& Hedley (1984) & 0.07 \\
\hline
\end{tabular}

Table 11. Recommended values of $k_{2}$ for equation (51).

\begin{tabular}{|l|c|}
\hline \multicolumn{1}{|c|}{ Investigator } & $\boldsymbol{k}_{2}$ \\
\hline Kneen \& Strauss (1969) & $3.79 \times 10^{-4}$ \\
\hline Liu \& Agarwal (1974) & $6 \times 10^{-4}$ \\
\hline Wood (1981b) & $4.5 \times 10^{-4}$ \\
\hline Papavergos \& Hedley (1984) & $3.5 \times 10^{-4}$ \\
\hline
\end{tabular}

Table 12. Recommended values of $k_{3}$ for equation (52).

\begin{tabular}{|l|c|}
\hline \multicolumn{1}{|c|}{ Investigator } & $\boldsymbol{k}_{3}$ \\
\hline Wood (1981b) & 0.13 \\
\hline Davies (1983) & 0.30 \\
\hline Papavergos \& Hedley (1984) & 0.18 \\
\hline Fan \& Ahmadi (1993) & 0.14 \\
\hline
\end{tabular}

Table 13. Summary of assumptions for free-flight model by different investigators.

\begin{tabular}{|c|c|c|c|c|}
\hline Investigators & $\underset{\xi_{p}}{\text { Particle eddy diffusivity, }}$ & $\begin{array}{c}\text { Eddy } \\
\text { viscosity } \\
\text { (Table 2.8), } \\
\xi_{a}\end{array}$ & $\begin{array}{c}\text { Dimensionless } \\
\text { capture } \\
\text { distance, } \\
\Delta^{+}\end{array}$ & $\begin{array}{c}\text { Dimensionless } \\
\text { particle } \\
\text { velocity at } \Delta^{+}, \\
v_{p y, \Delta^{+}}^{+}\end{array}$ \\
\hline $\begin{array}{l}\text { Friedlander \& } \\
\text { Johnstone } \\
\text { (1957) }\end{array}$ & $\xi_{p}=\xi_{a}$ & $\begin{array}{l}\text { Lin et al. } \\
\text { (1953) }\end{array}$ & $S^{+}$ & 0.9 \\
\hline Davies (1966b) & $\xi_{p}=\xi_{a}$ & Davies (1966a) & $S^{+}+r_{p}^{+}$ & $\Delta^{+} /\left(\Delta^{+}+10\right)$ \\
\hline $\begin{array}{l}\text { Liu \& Ilori } \\
\text { (1974) }\end{array}$ & $\xi_{p}=\xi_{a}+v \tau^{+} v_{r m s}^{\prime+2}$ & Owen (1960) & $S^{+}+r_{p}^{+}$ & $\Delta^{+} /\left(\Delta^{+}+10\right)$ \\
\hline Browne (1974) & $\xi_{p}=\xi_{a}$ & Davies (1966a) & $S^{+}+r_{p}^{+}+k^{+}+\sigma_{k}^{+}-e^{+}$ & $\Delta^{+} /\left(\Delta^{+}+10\right)$ \\
\hline $\begin{array}{l}\text { El-Shobokshy } \\
\text { \& Ismail } \\
(1980)\end{array}$ & $\xi_{p}=\xi_{a}+v \tau^{+} v_{r m s}^{\prime+2}$ & $\begin{array}{l}\text { Lin et al. } \\
(1953)\end{array}$ & $S^{+}+r_{p}^{+}+k^{+}+\sigma_{k}^{+}-e^{+}$ & $\Delta^{+} /\left(\Delta^{+}+10\right)$ \\
\hline Wood (1981a) & $\xi_{p}=\xi_{a}$ & $\begin{array}{l}\text { Lin et al. } \\
\text { (1953) }\end{array}$ & $S^{+}+r_{p}^{+}+k^{+}-e^{+}$ & 0.69 \\
\hline $\begin{array}{l}\text { Im \& } \\
\text { Ahluwalia } \\
(1989)\end{array}$ & $\xi_{p}=\xi_{a}\left[1+\frac{\exp \left(-\tau_{f} / \tau_{p}+1\right)-1}{1.72\left[\left(\tau_{f} / \tau_{p}\right)^{2}-1\right]}\right]$ & $\begin{array}{c}\text { Cebeci \& } \\
\text { Smith (1974) }\end{array}$ & $S^{+}+e^{+}$ & $0.8\left[\frac{\tau_{f}}{\tau_{f}+\tau_{p}}\right]^{1 / 2}$ \\
\hline
\end{tabular}




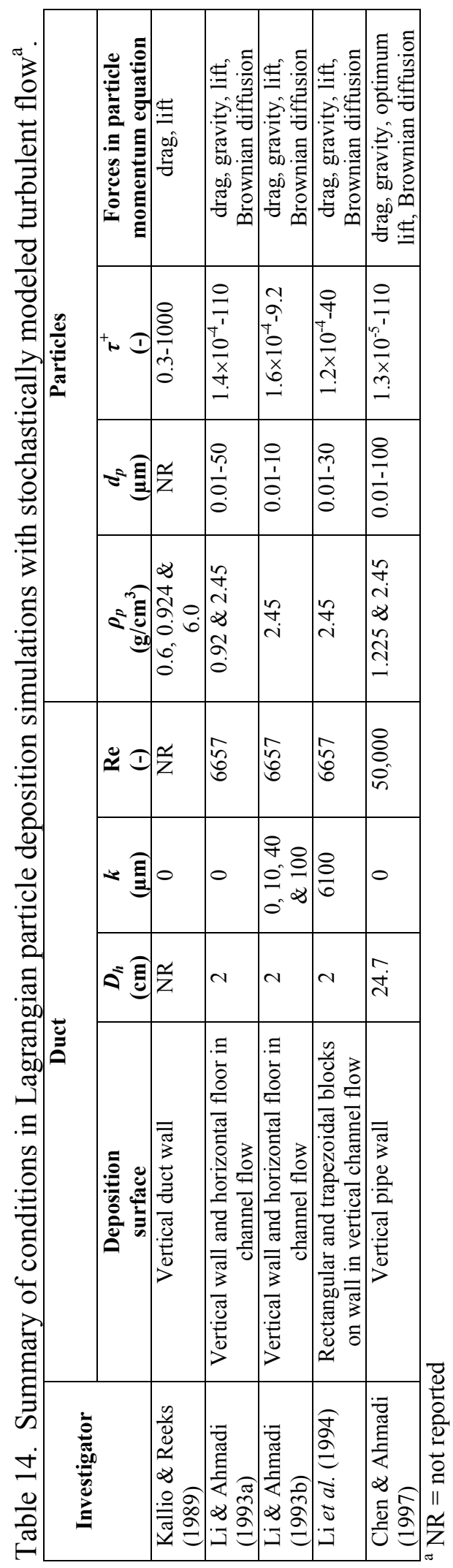




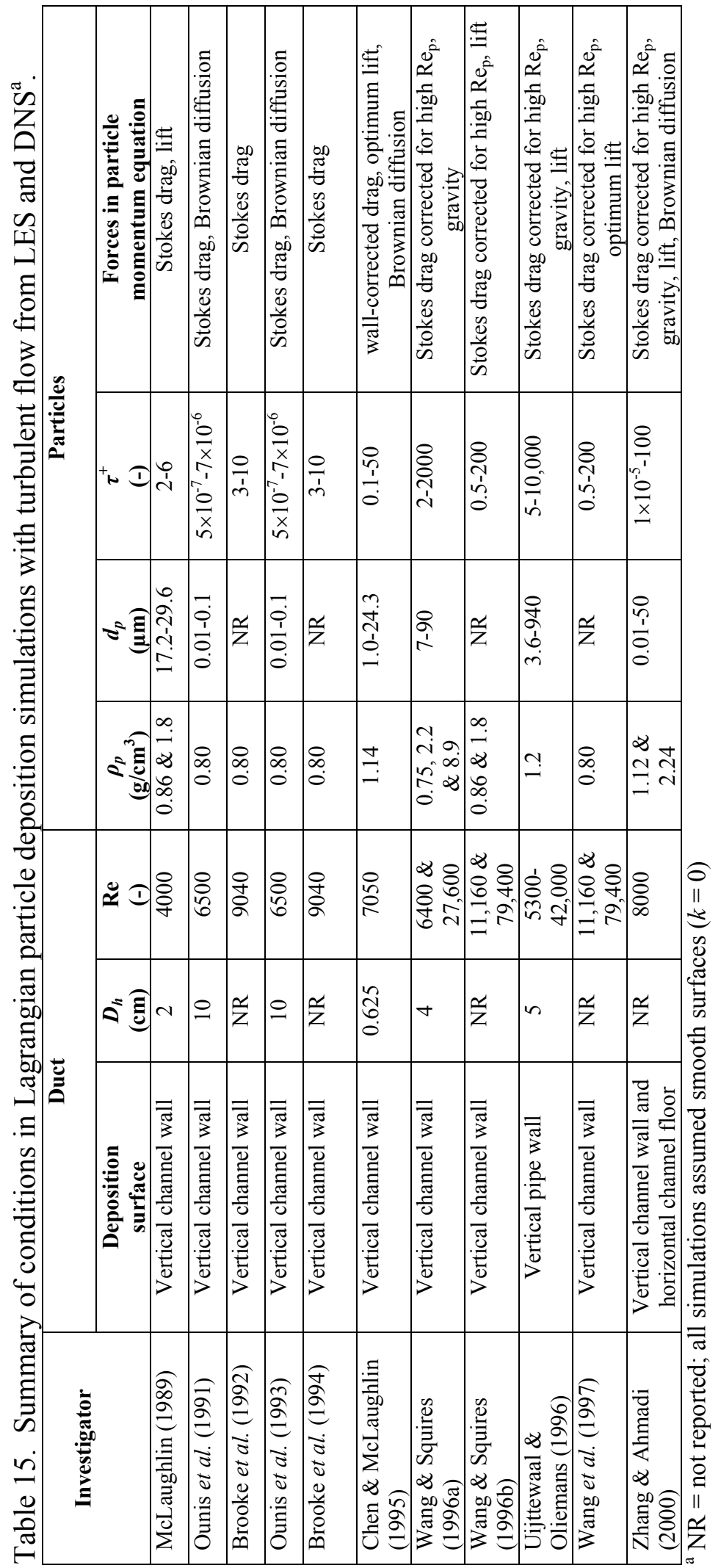




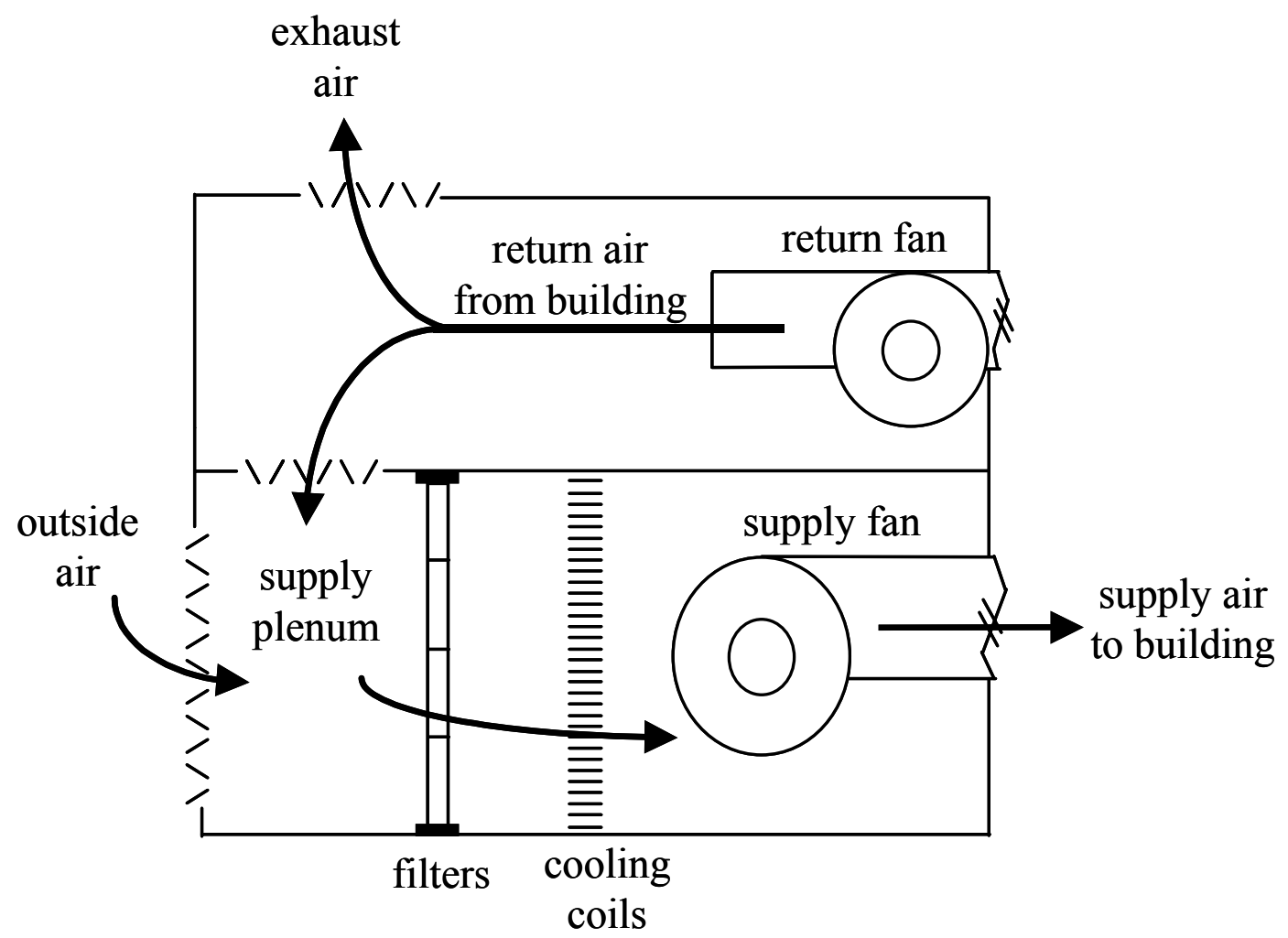

Figure 1. Schematic diagram of airflow through the mechanical room for a typical HVAC system.

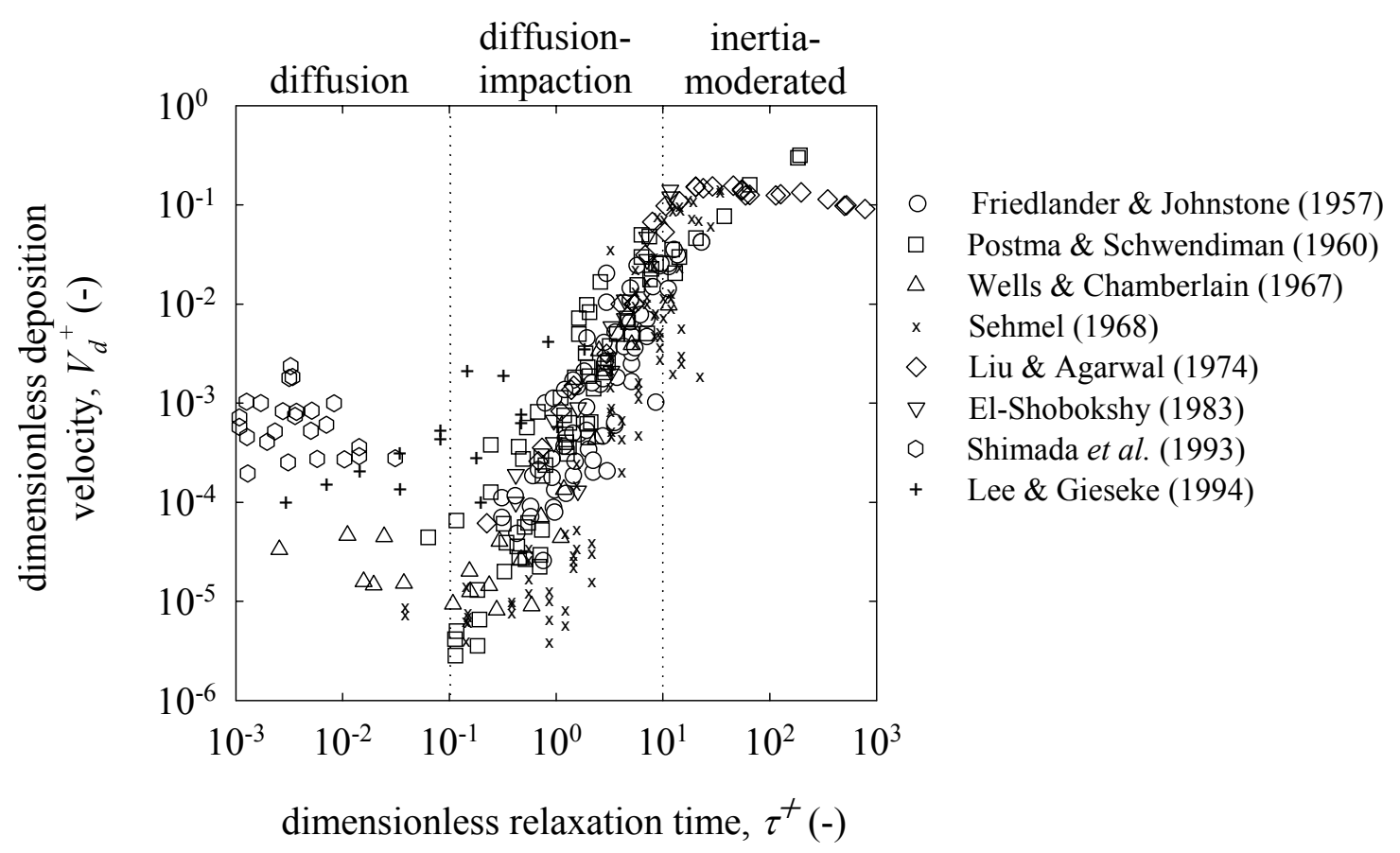

Figure 2. Experimental particle deposition data collected from turbulent flow through small vertical tubes by several researchers. 


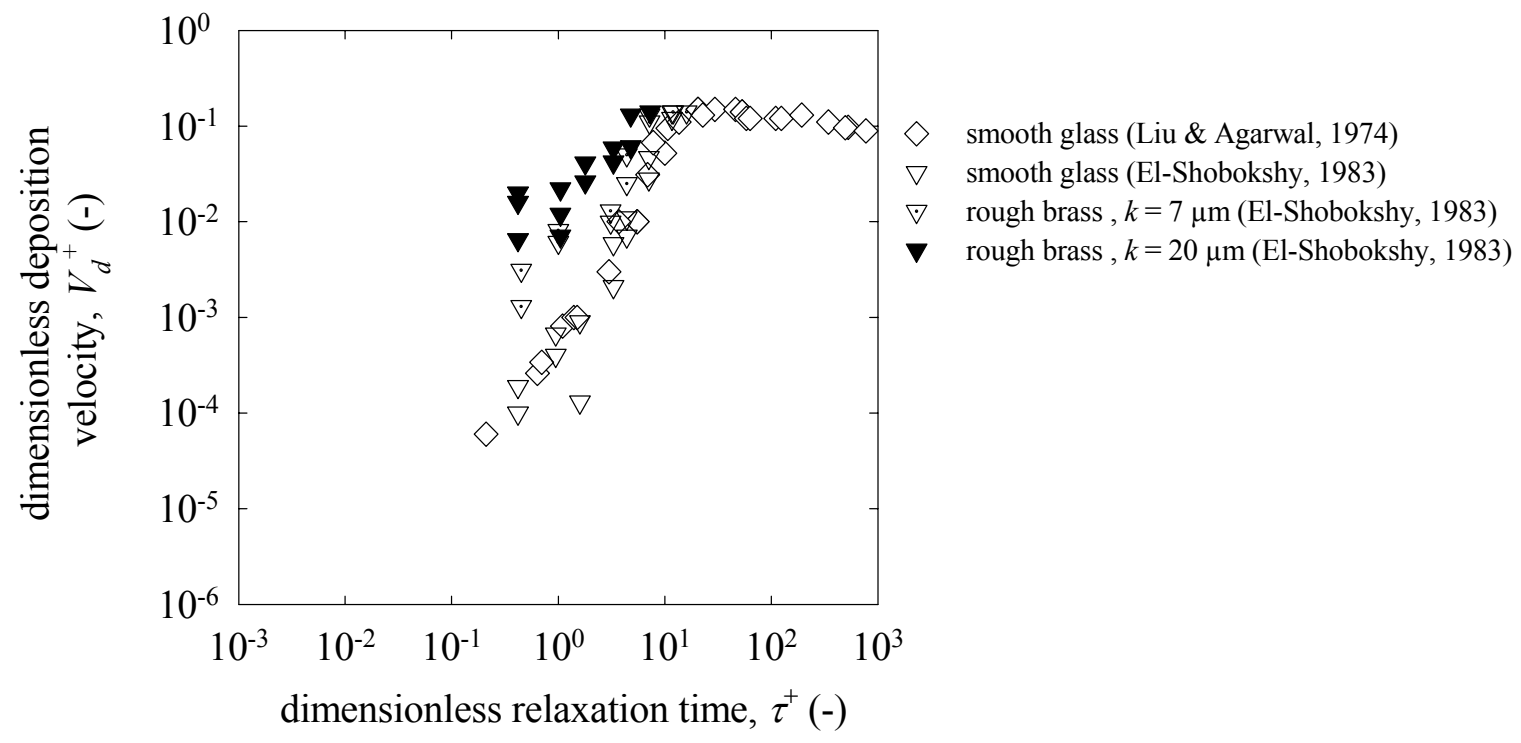

Figure 3. Experimental particle deposition data collected from flow through vertical smooth and rough tubes in the diffusion-impaction and inertia-moderated regimes.

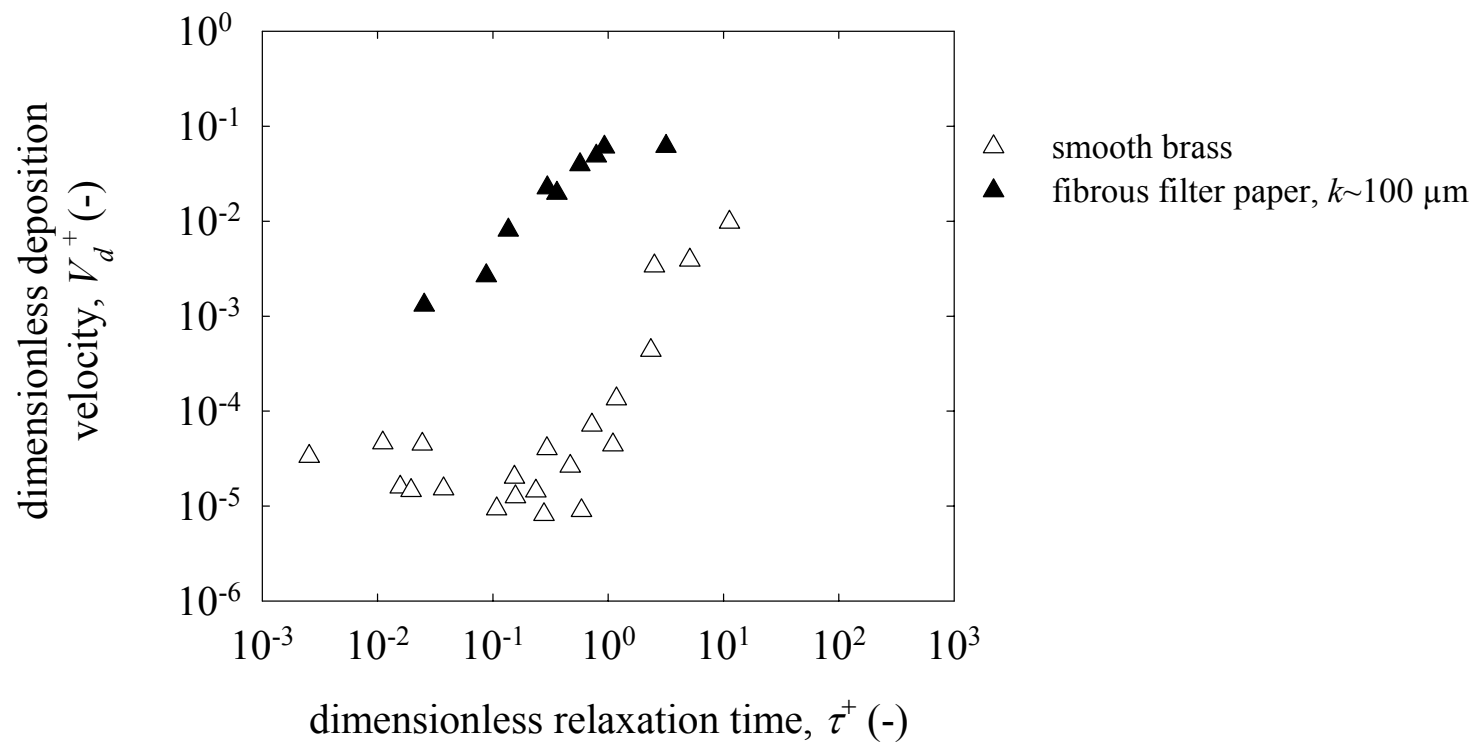

Figure 4. Experimental particle deposition data collected by Wells \& Chamberlain (1967) for particles depositing to vertically oriented smooth brass and fibrous filter paper with roughness length scale, $k$, of approximately $100 \mu \mathrm{m}$. 


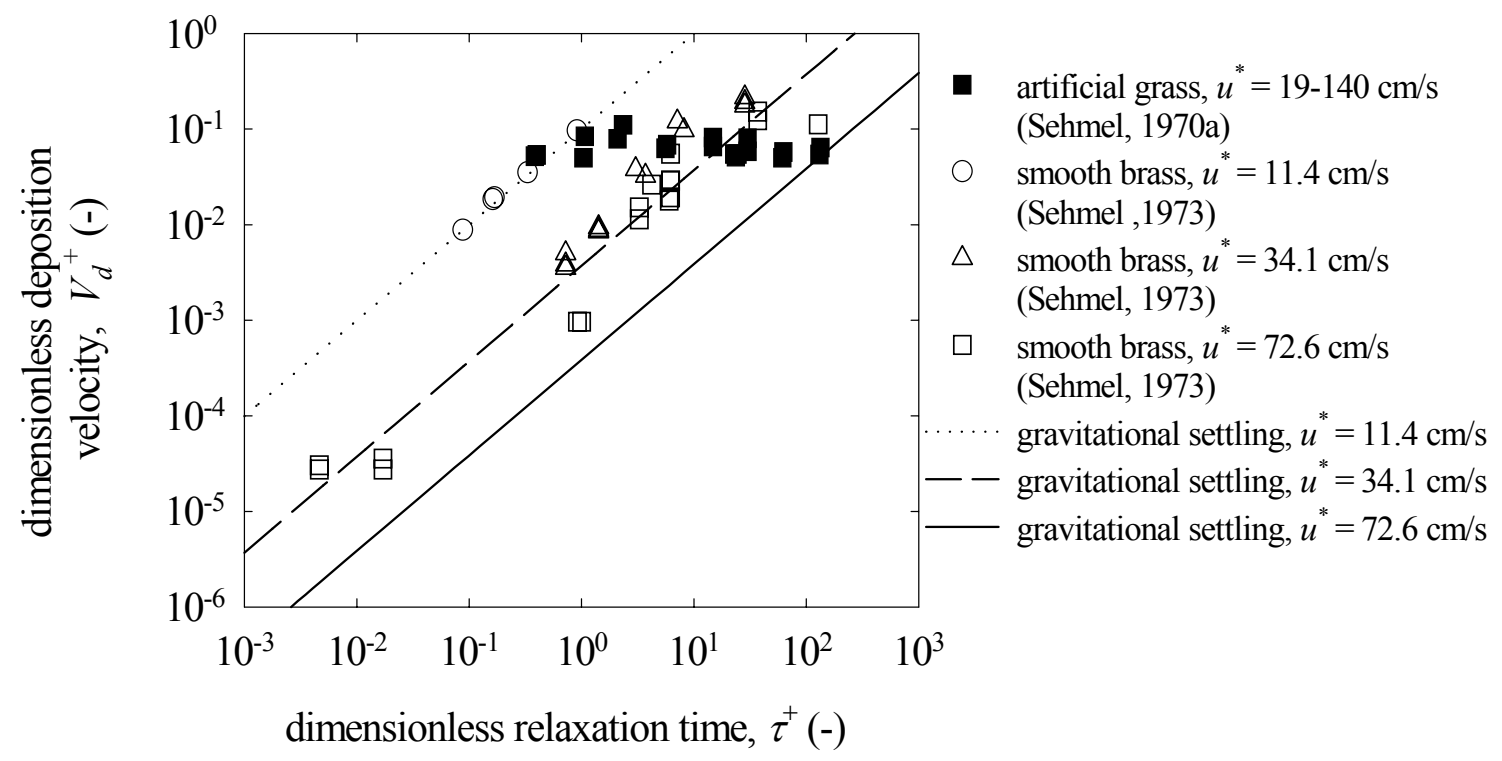

Figure 5. Experimental particle deposition data for particles depositing to a smooth duct floor and a duct floor covered with artificial grass at different values of the friction velocity, $u^{*}$.

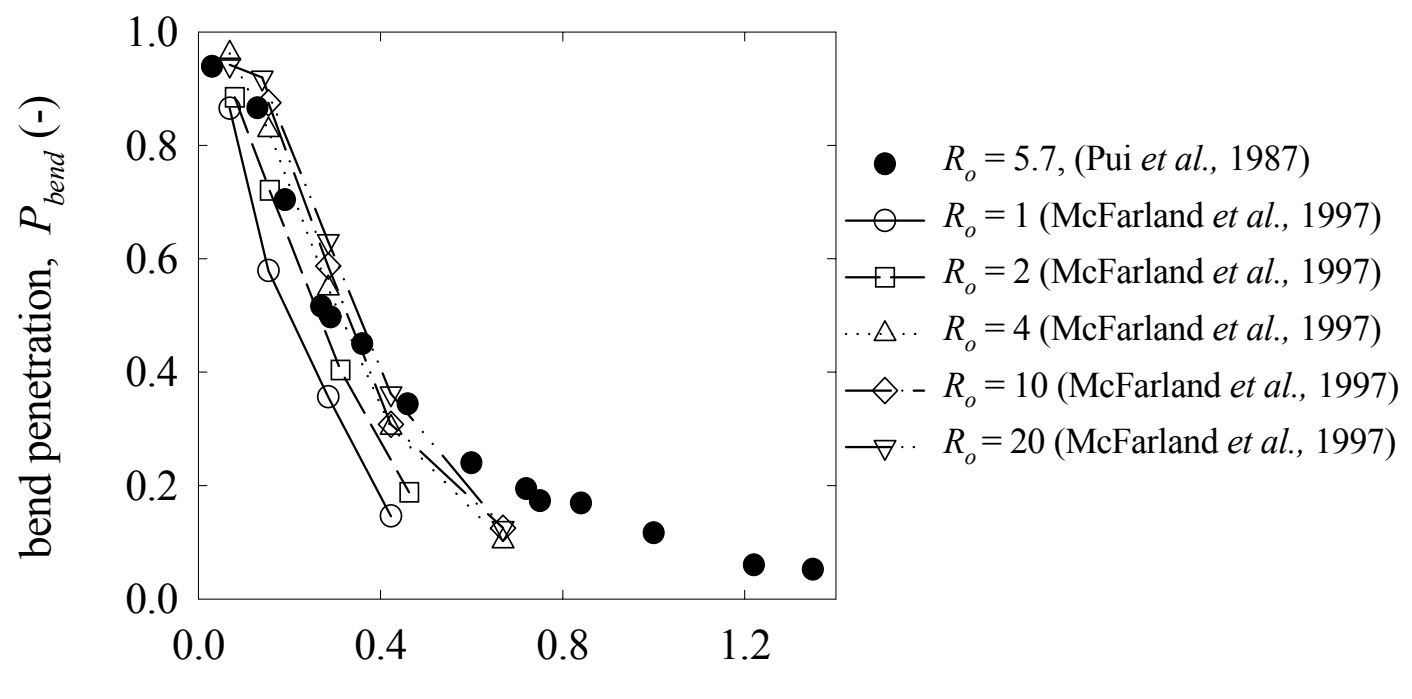

Stokes number, St (-)

Figure 6. Experimental data collected for particle penetration through 90-degree bends with turbulent flow and small tube diameters. 


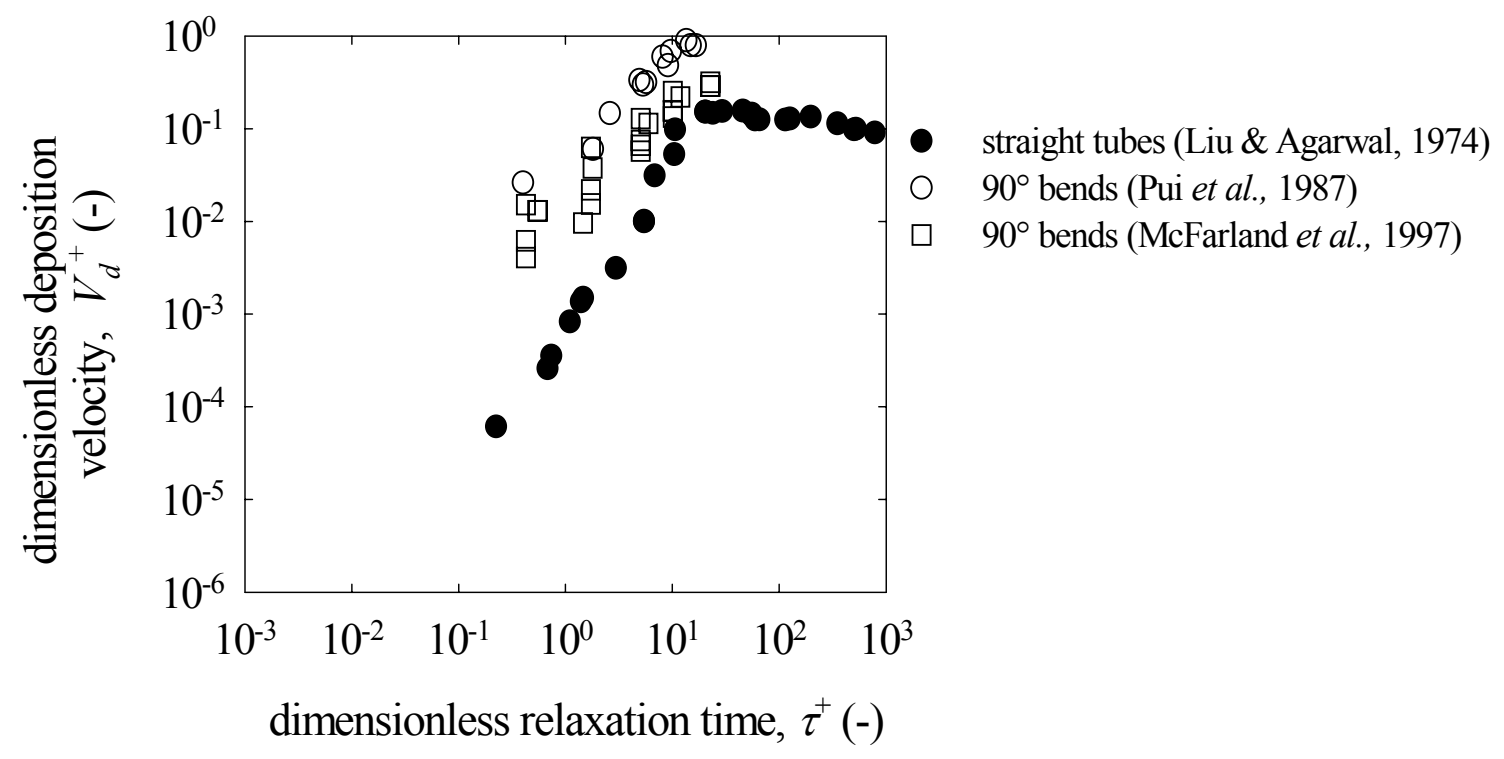

Figure 7. Experimental data collected for deposition in 90-degree bends with turbulent flow and small tube diameters compared to experimental data collected in straight tubes.

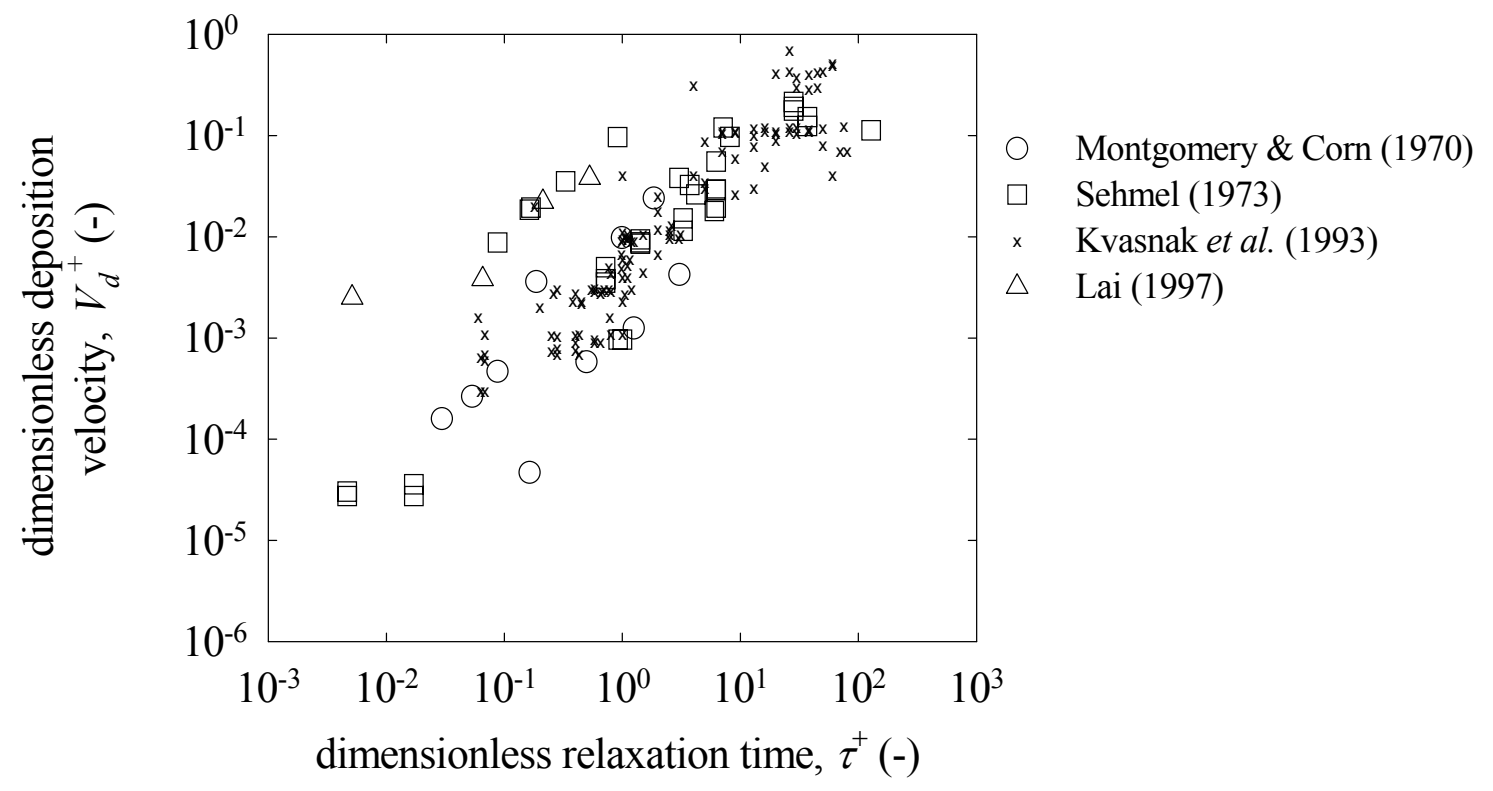

Figure 8. Experimental particle deposition data for particles depositing to the smooth floors of large ducts. 


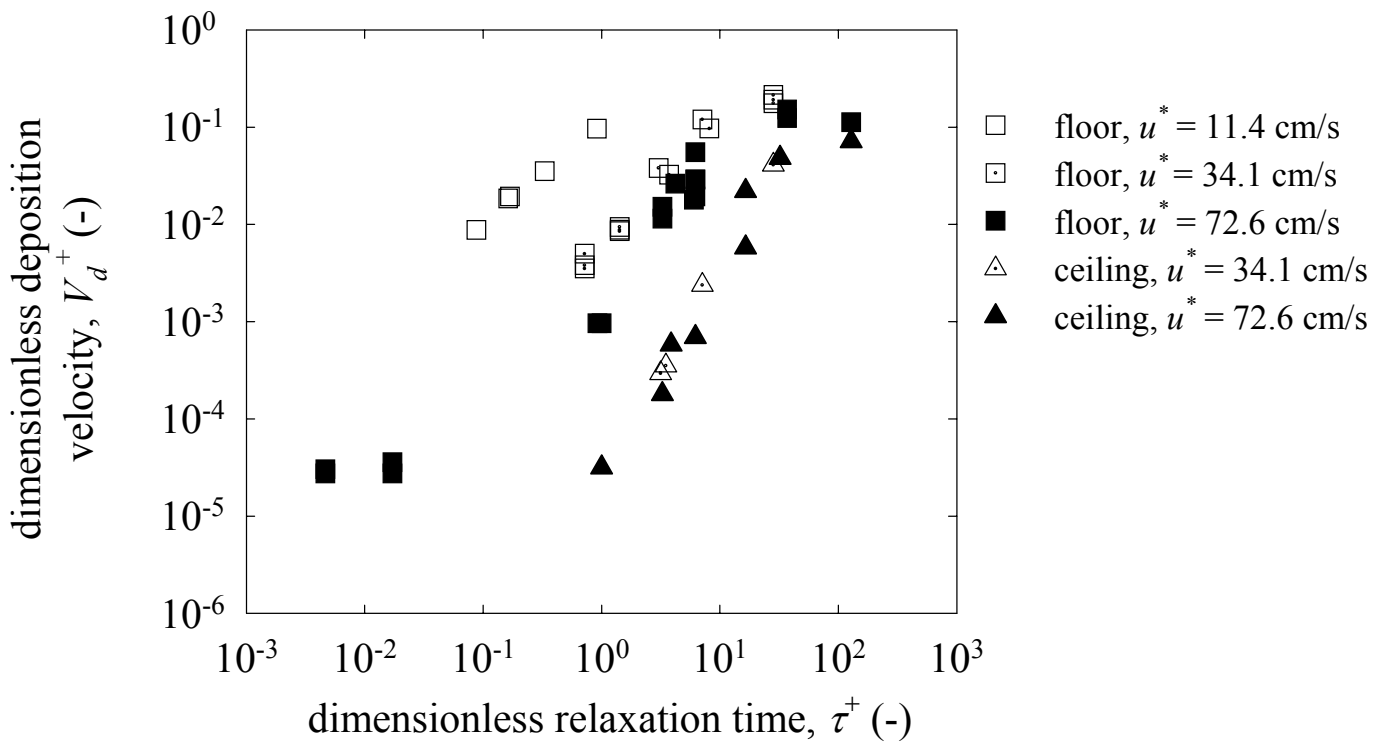

Figure 9. Experimental particle deposition data collected by Sehmel (1973) for particles depositing to the floor and ceiling of a horizontal duct at three different values of the friction velocity, $u^{*}$.

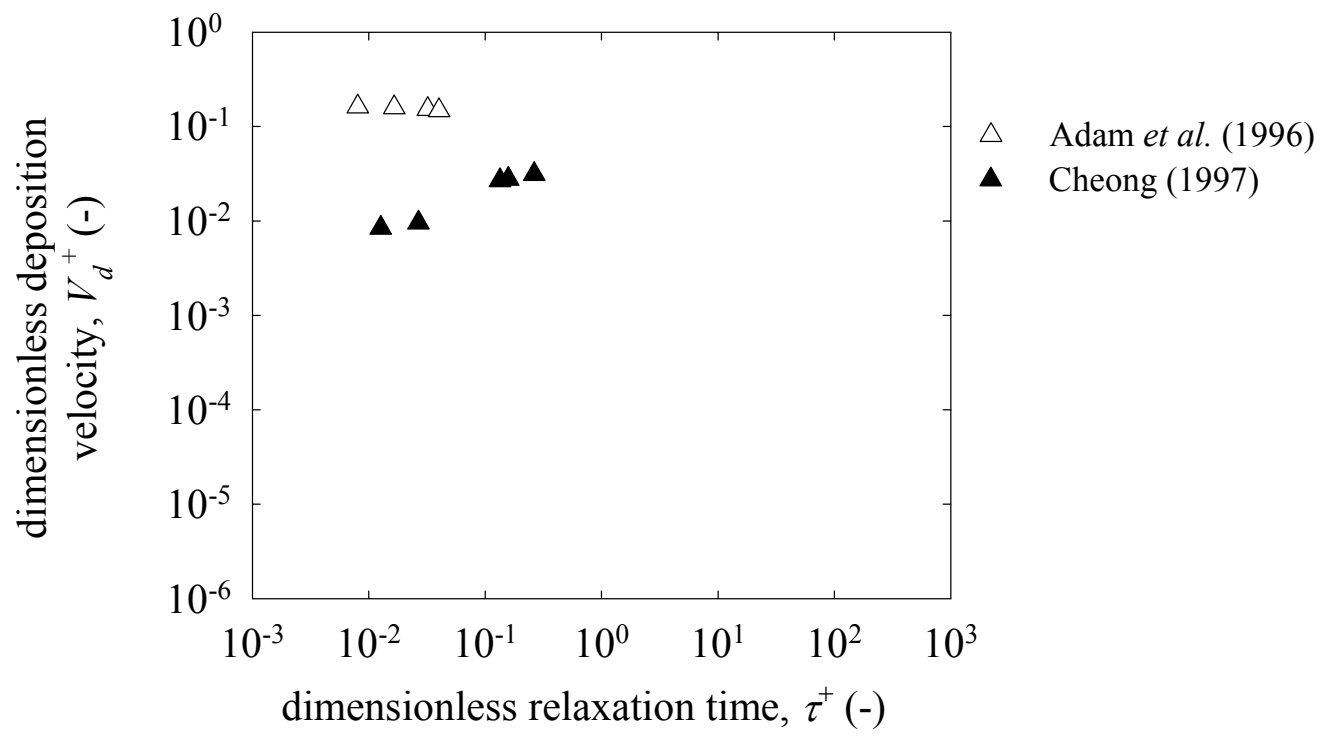

Figure 10. Particle deposition data for polydisperse aerosols in straight $30 \mathrm{~cm}$ square ducts measured by inferring deposition from differences in concentration measurements. 


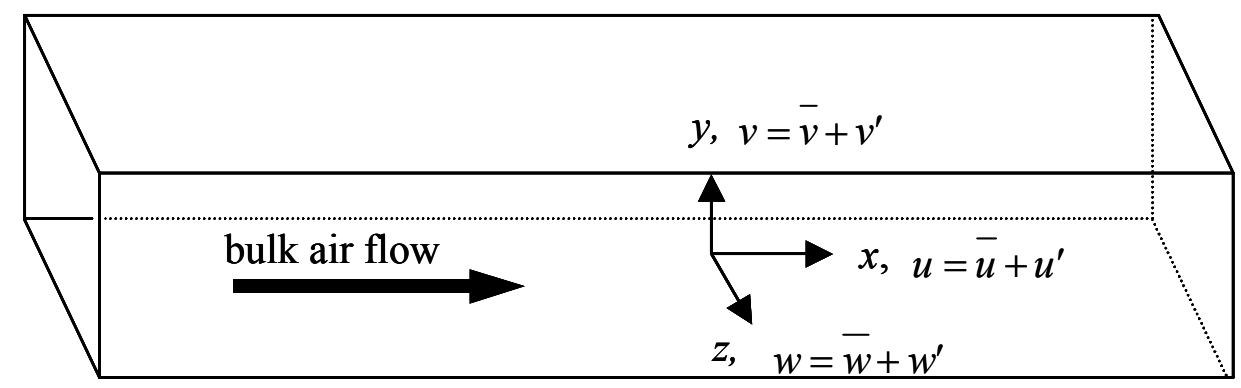

Figure 11. Definition of coordinate directions in turbulent duct flow 

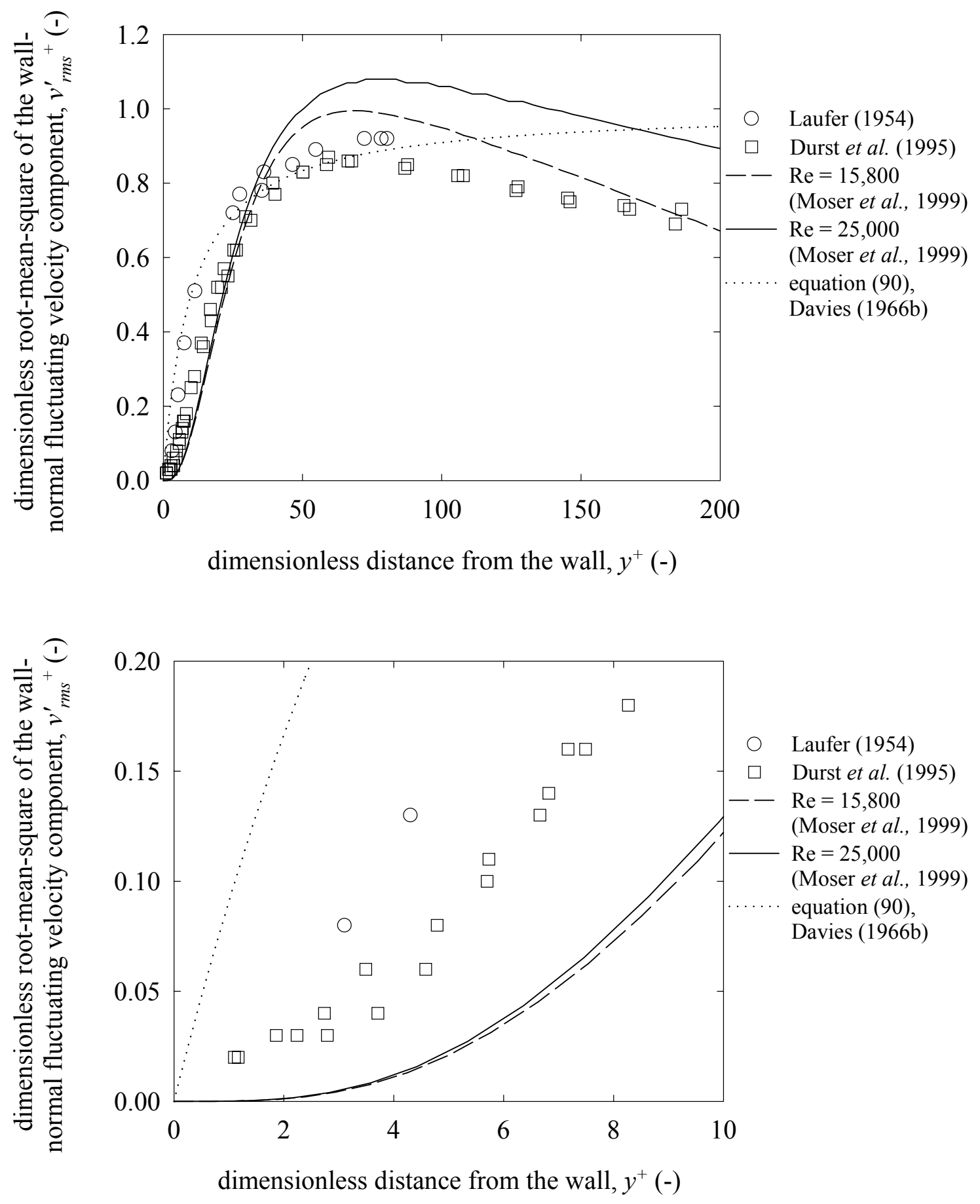

Figure 12. Profiles of $v_{r m s}^{\prime+}$ versus $y^{+}$derived from measurements in pipe flow and DNS of channel flow. The bottom panel shows the same data as the top panel with the axes scaled to focus attention near the wall. 


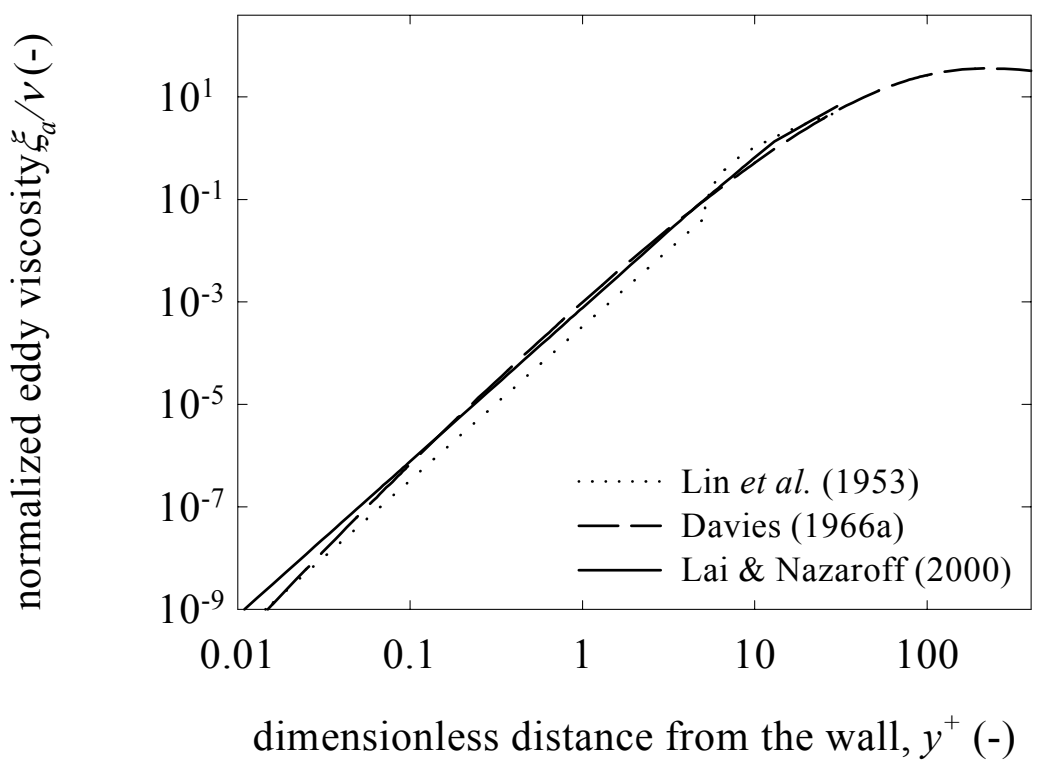

Figure 13. Comparison of correlations for eddy diffusivity versus dimensionless distance from a wall.

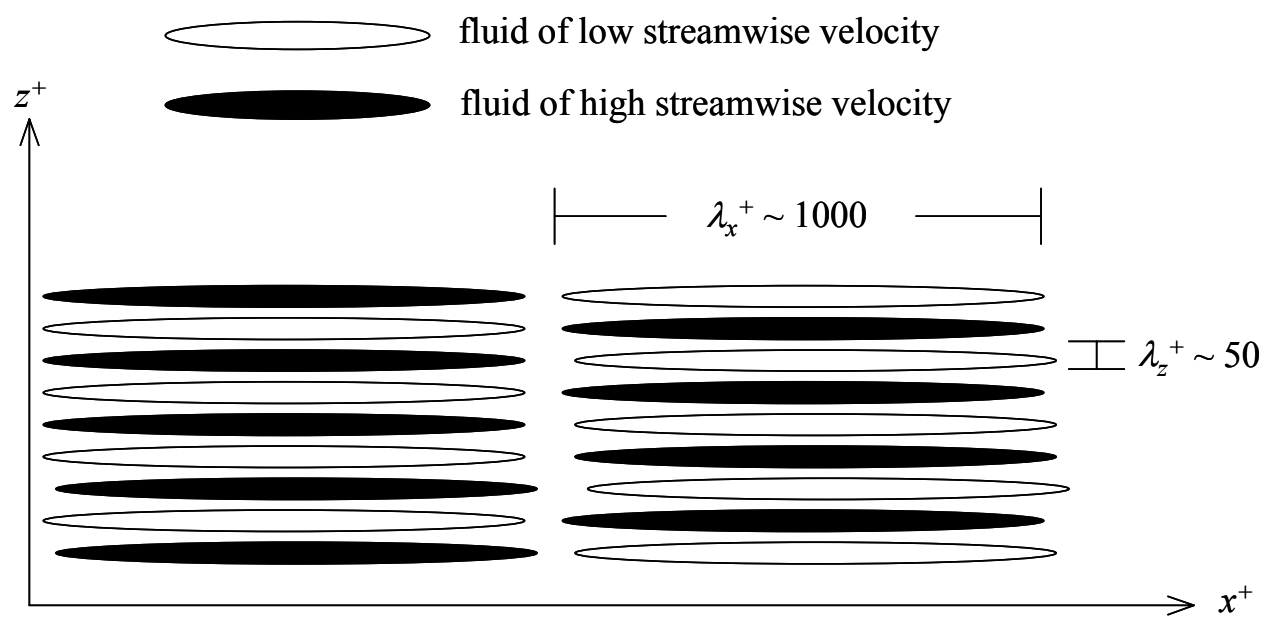

Figure 14. Approximate instantaneous arrangement and magnitudes of alternating lowspeed and high-speed streaks of fluid near a wall in turbulent flow. 


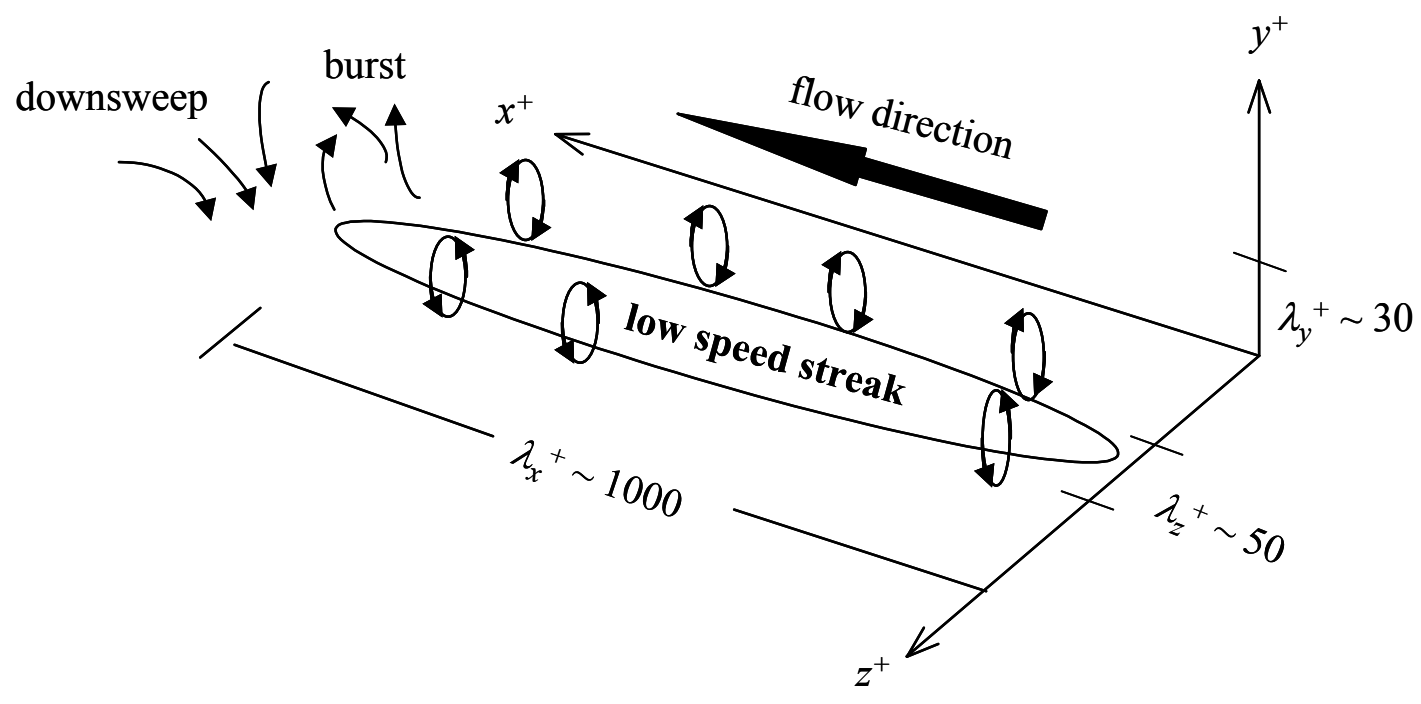

Figure 15. Schematic of near-wall turbulence illustrating the association between lowspeed streaks, streamwise vortices, bursts and down sweeps.

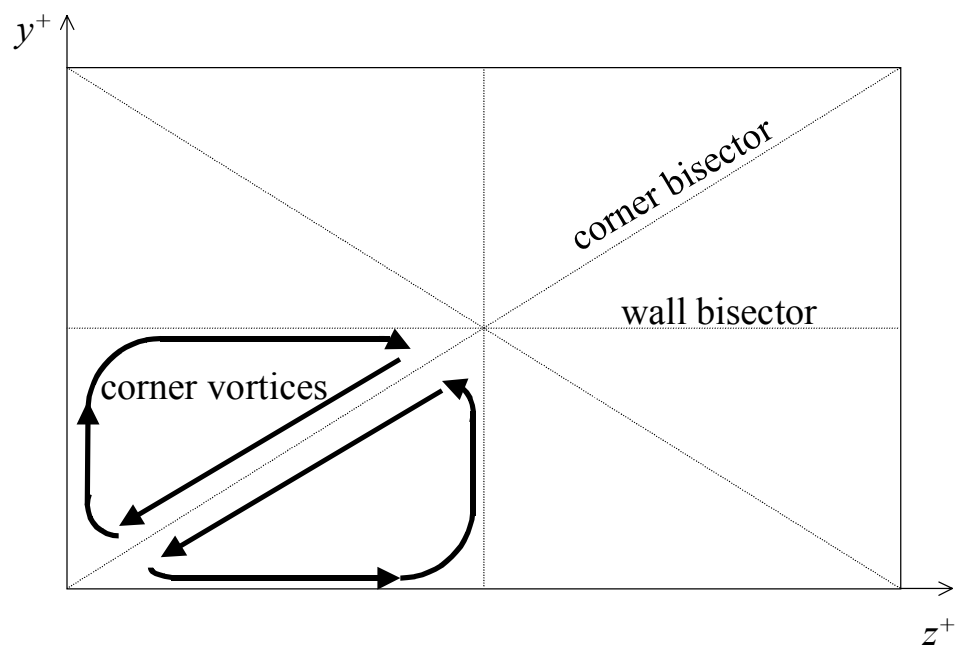

Figure 16. Secondary flow in the $y-z$ plane of a straight rectangular duct with fully developed turbulent flow. 


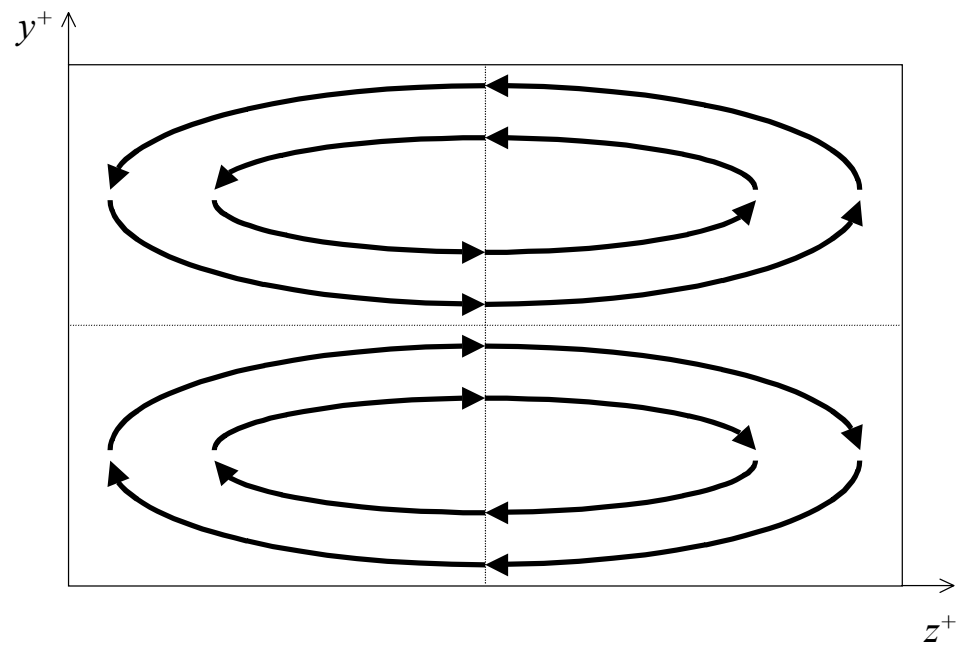

Figure 17. Secondary flow established in the $y-z$ plane in a leftward turning bend with the outside of the bend to the right.

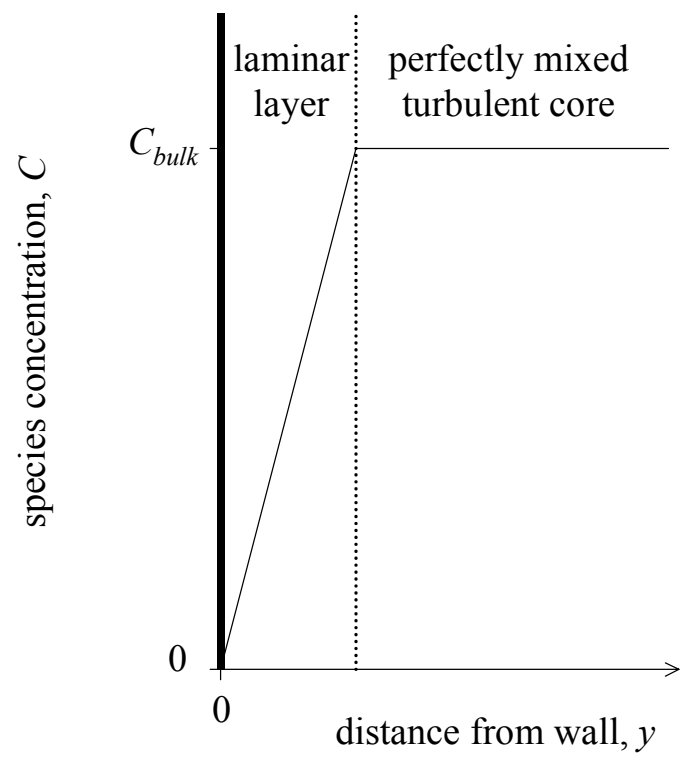

Figure 18. Concentration profile of a diffusive species based on the assumptions of the film model. 


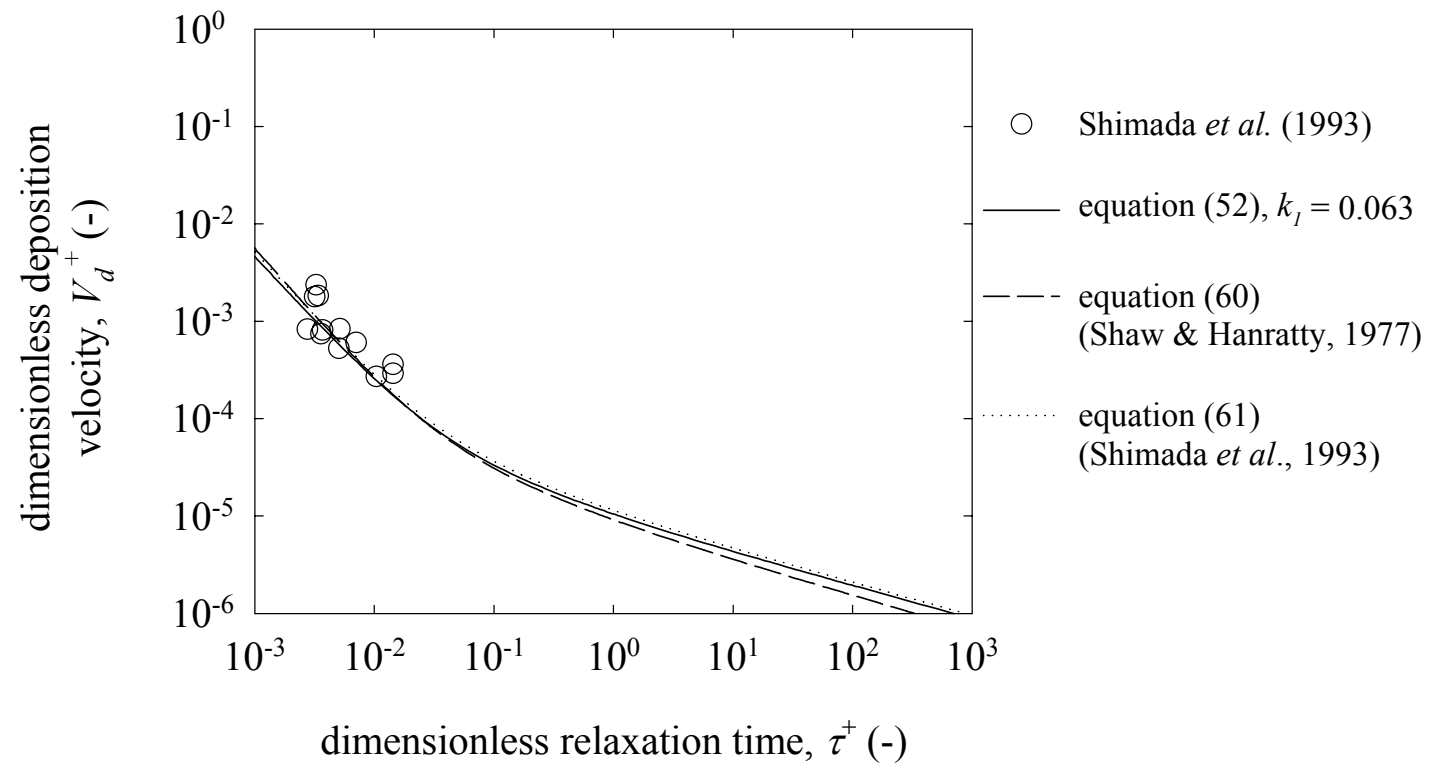

Figure 19. Comparison of empirical expressions for particle dimensionless deposition velocities in the diffusion regime.

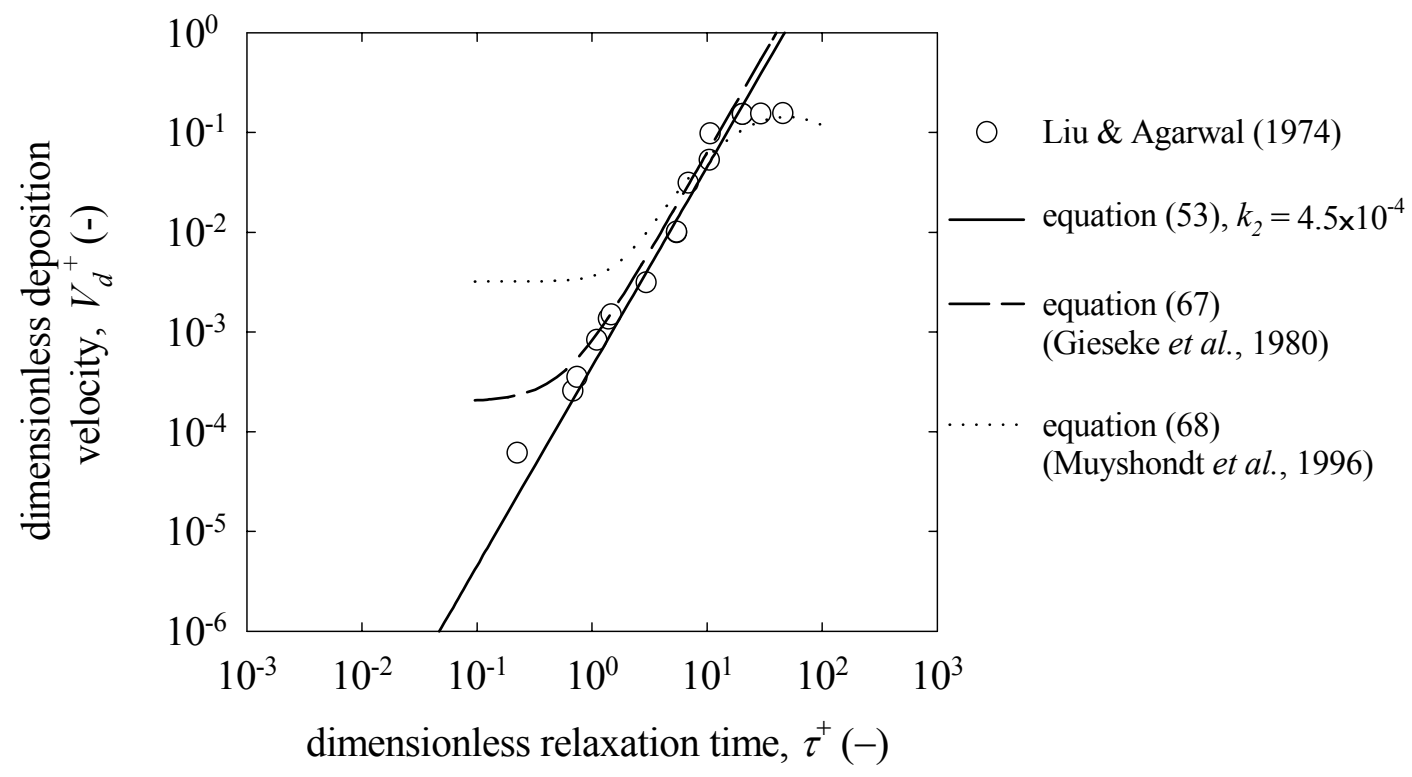

Figure 20. Comparison of empirical expressions for particle dimensionless deposition velocities in the diffusion-impaction regime. 


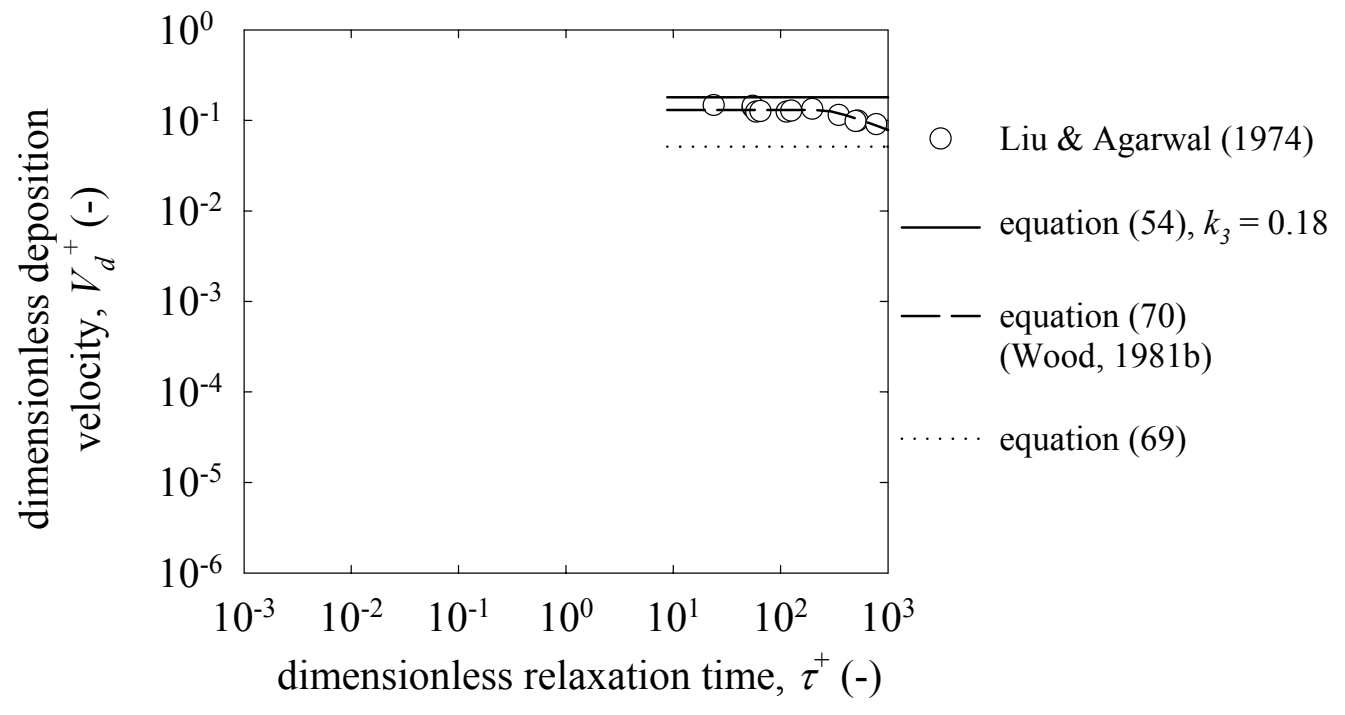

Figure 21. Comparison of empirical expressions for particle dimensionless deposition velocities in the inertia-moderated regime.

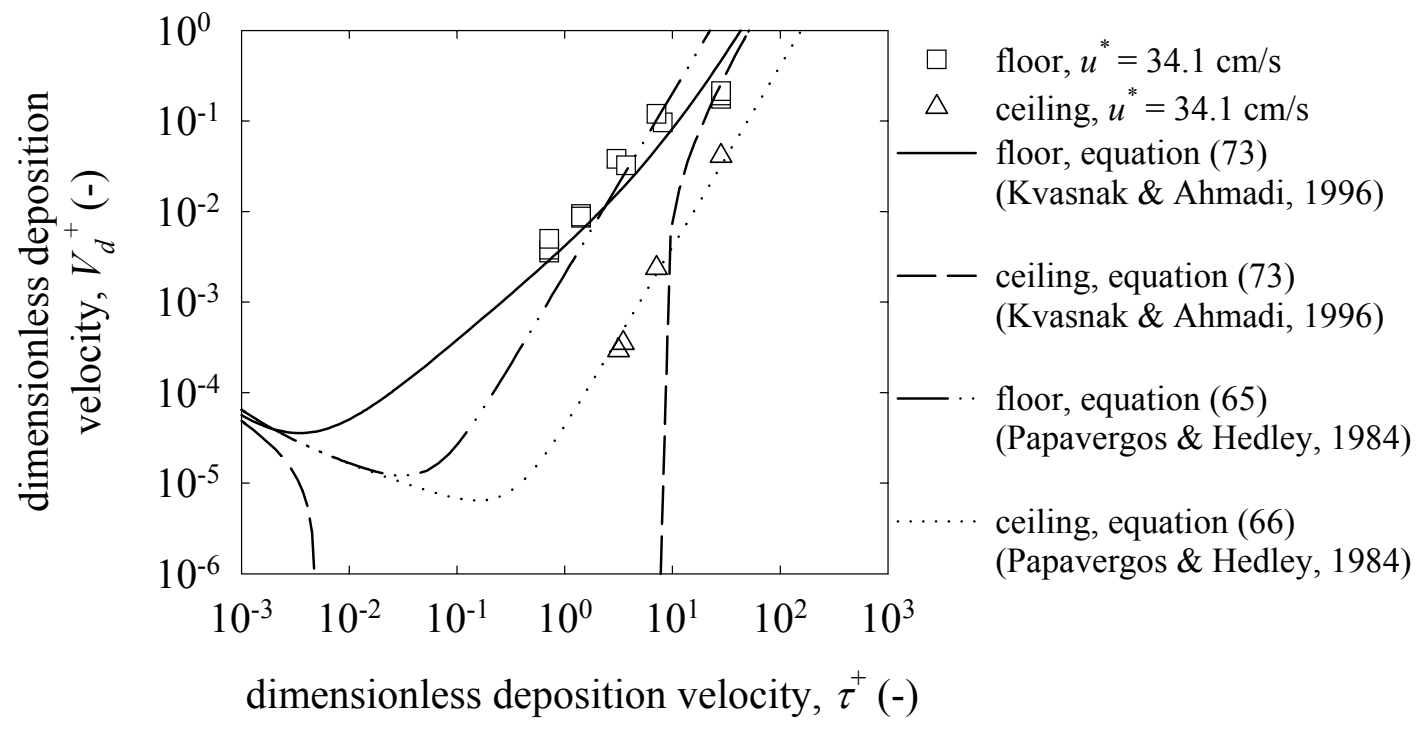

Figure 22. Comparison of empirical expressions for particle dimensionless deposition velocities to horizontal floor and ceiling surfaces. 


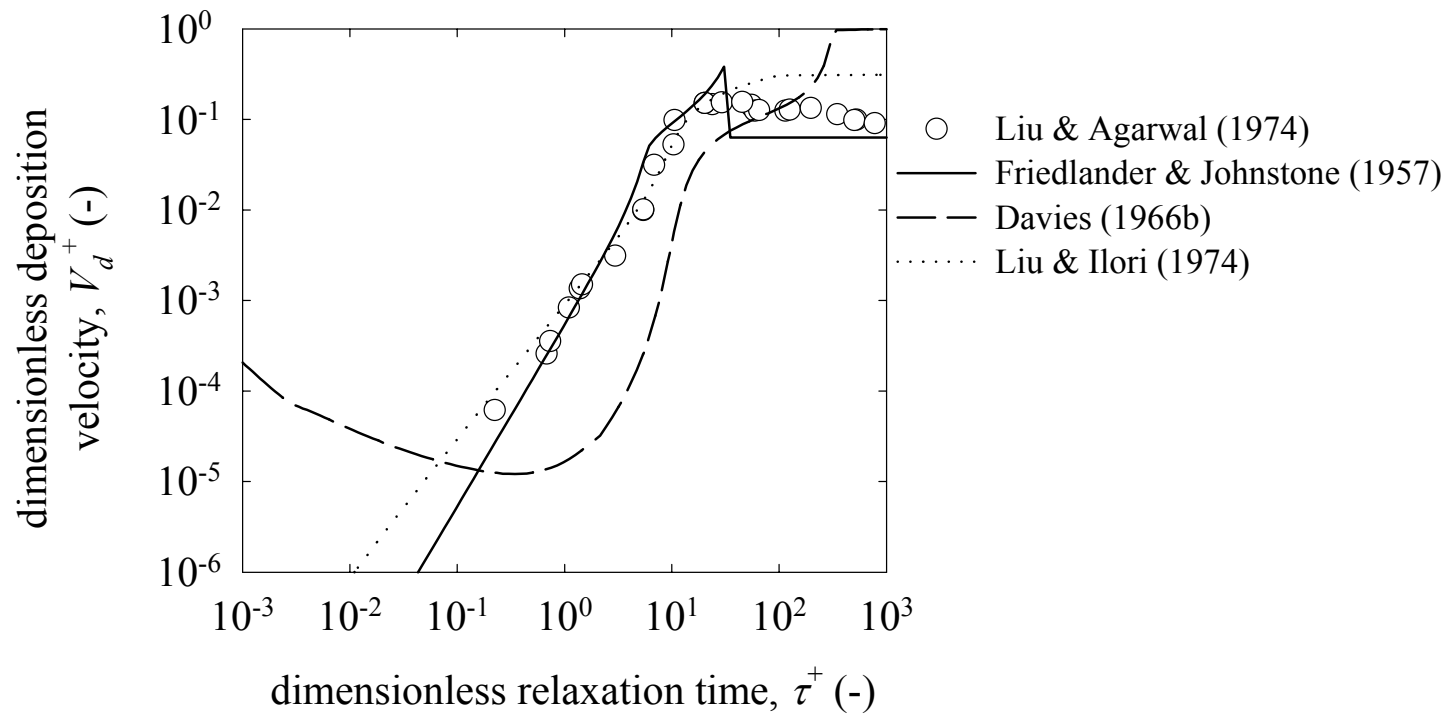

Figure 23. Comparison of free flight models in the case of deposition to a smooth vertical surface.

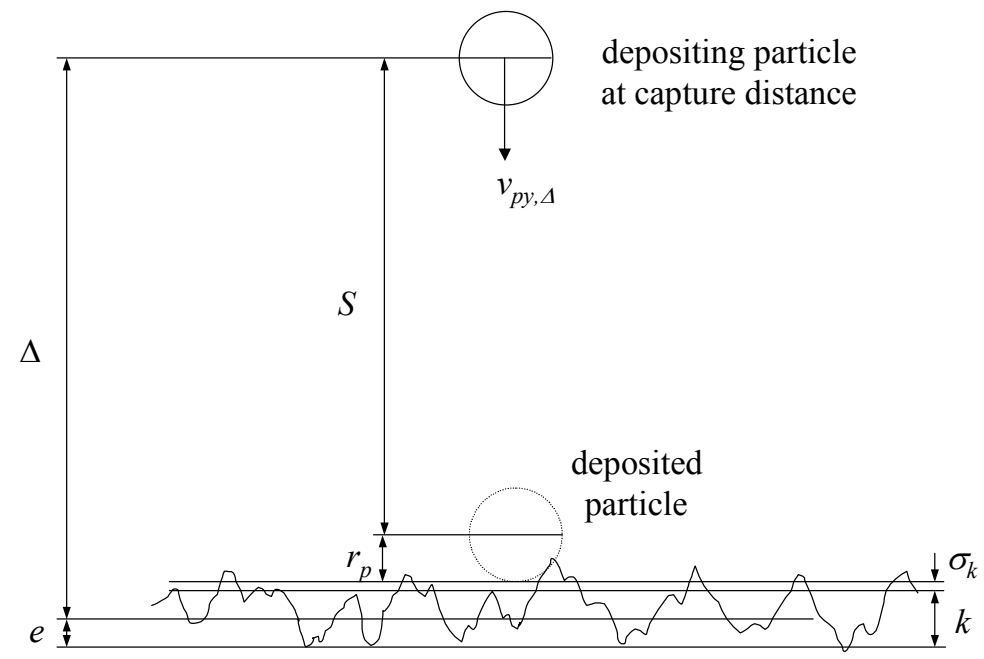

Figure 24. Description of parameters to determine the particle capture distance to a rough surface by the method recommended by Browne (1974). 


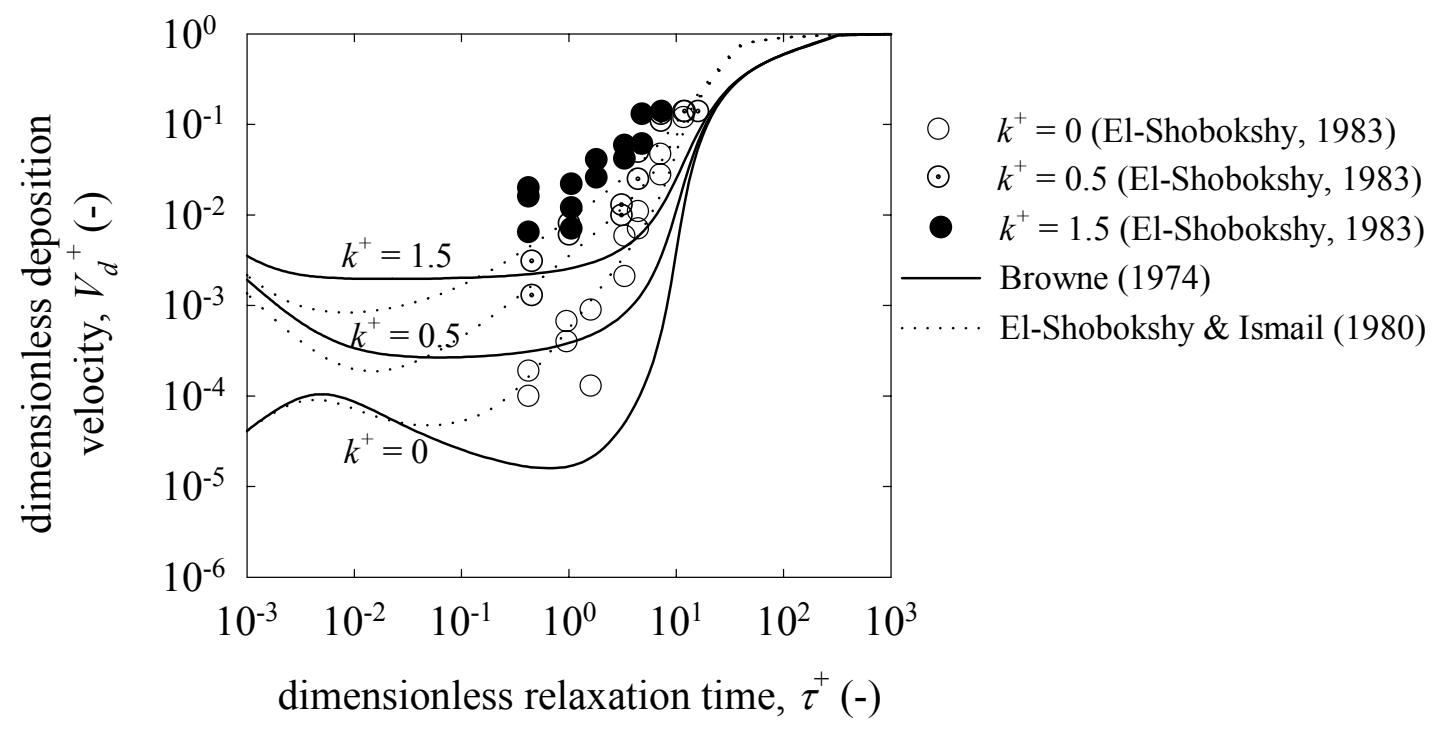

Figure 25. Comparison of free-flight models of Browne (1974) and El-Shobokshy \& Ismail (1980) with experimental data in the case of deposition to a rough vertical surface.

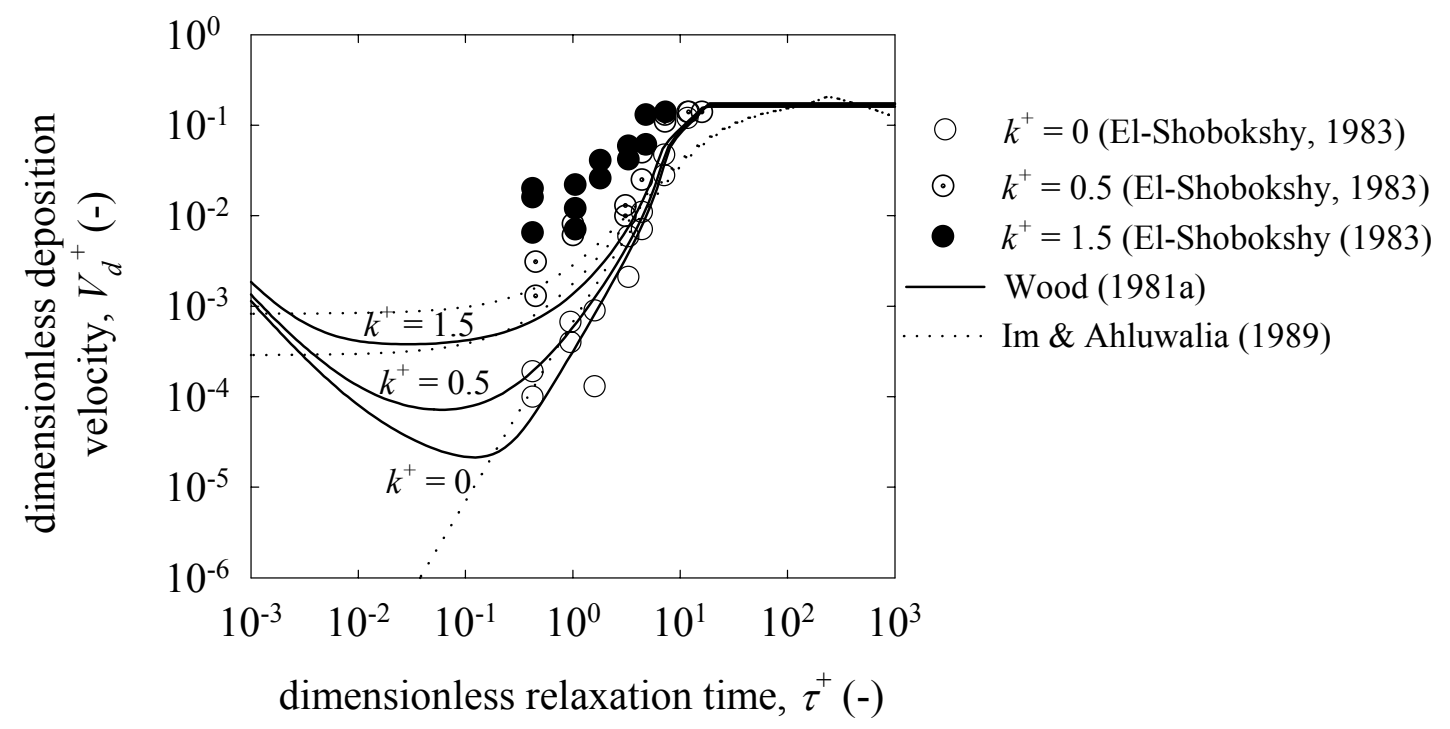

Figure 26. Comparison of free-flight models of Wood (1981a) and Im \& Ahluwalia (1989) with experimental data in the case of deposition to a rough vertical surface. 


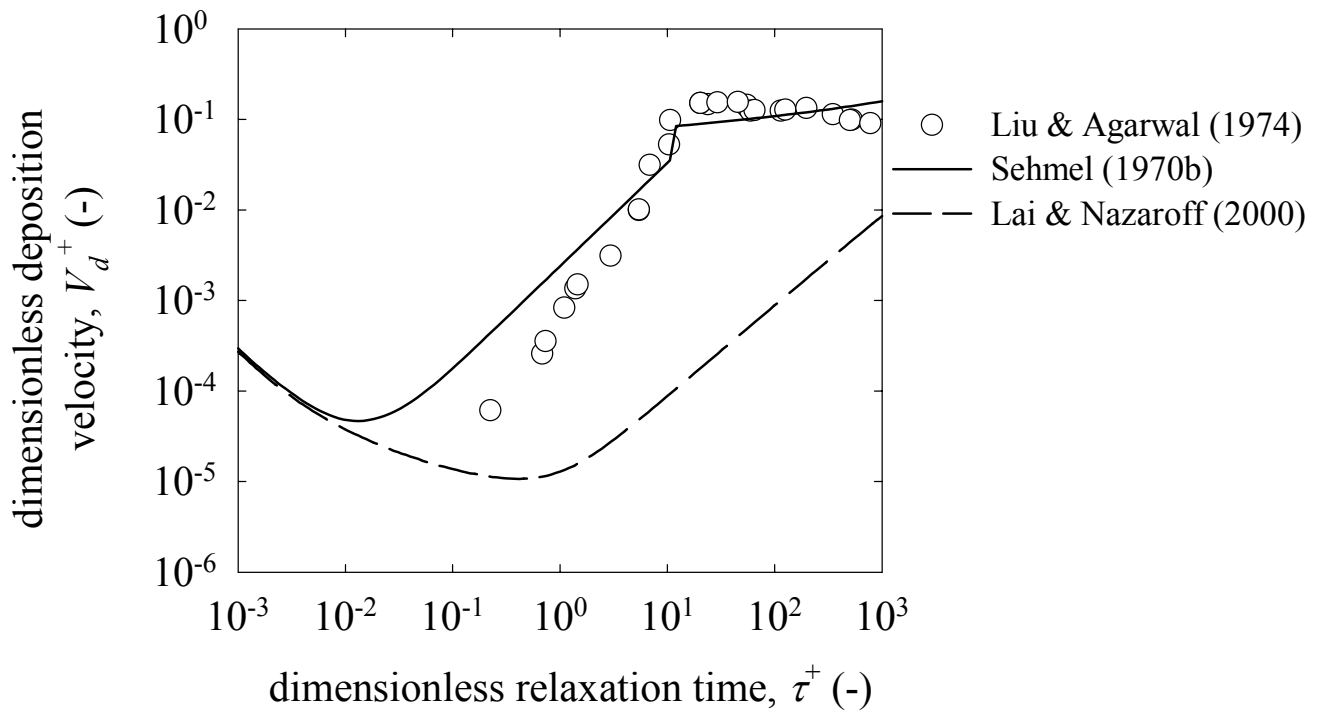

Figure 27. Comparison of gradient diffusion models in the case of a smooth vertical surface.

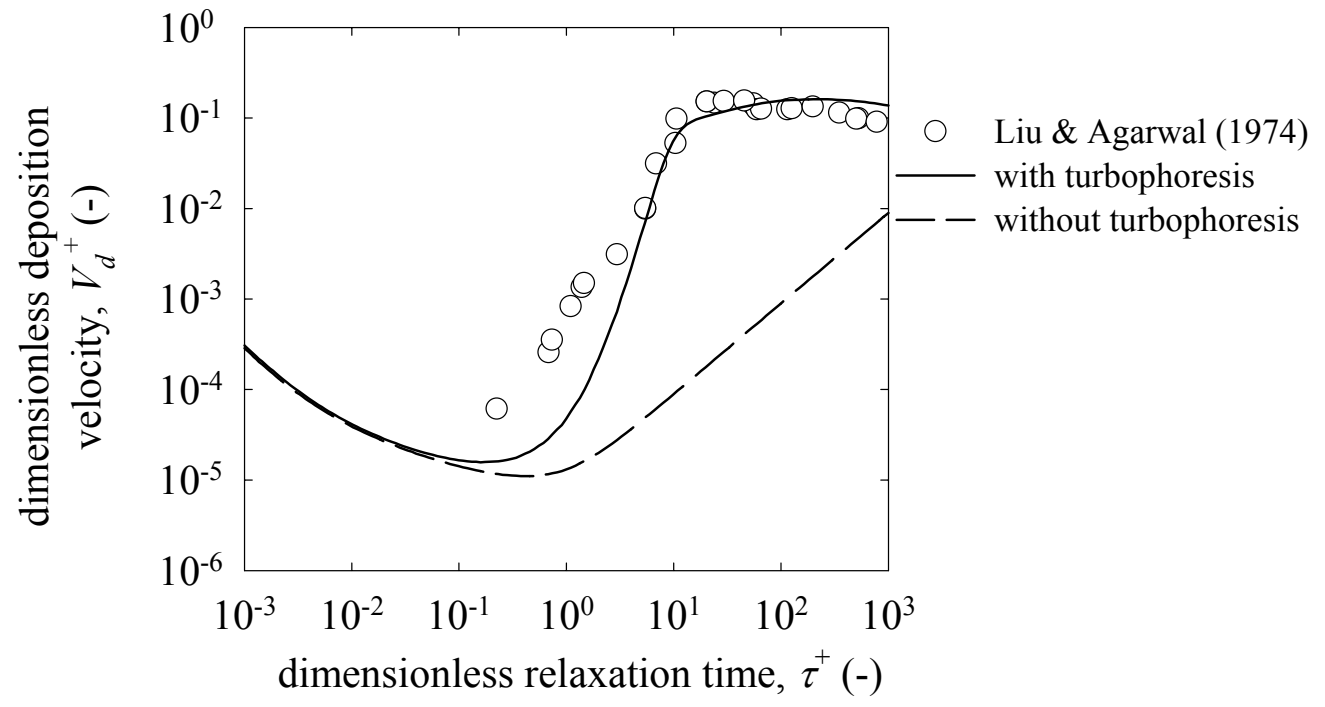

Figure 28. Comparison of the turbophoretic model of Guha (1997) in the case of a smooth vertical surface including and excluding the effect of turbophoresis. 


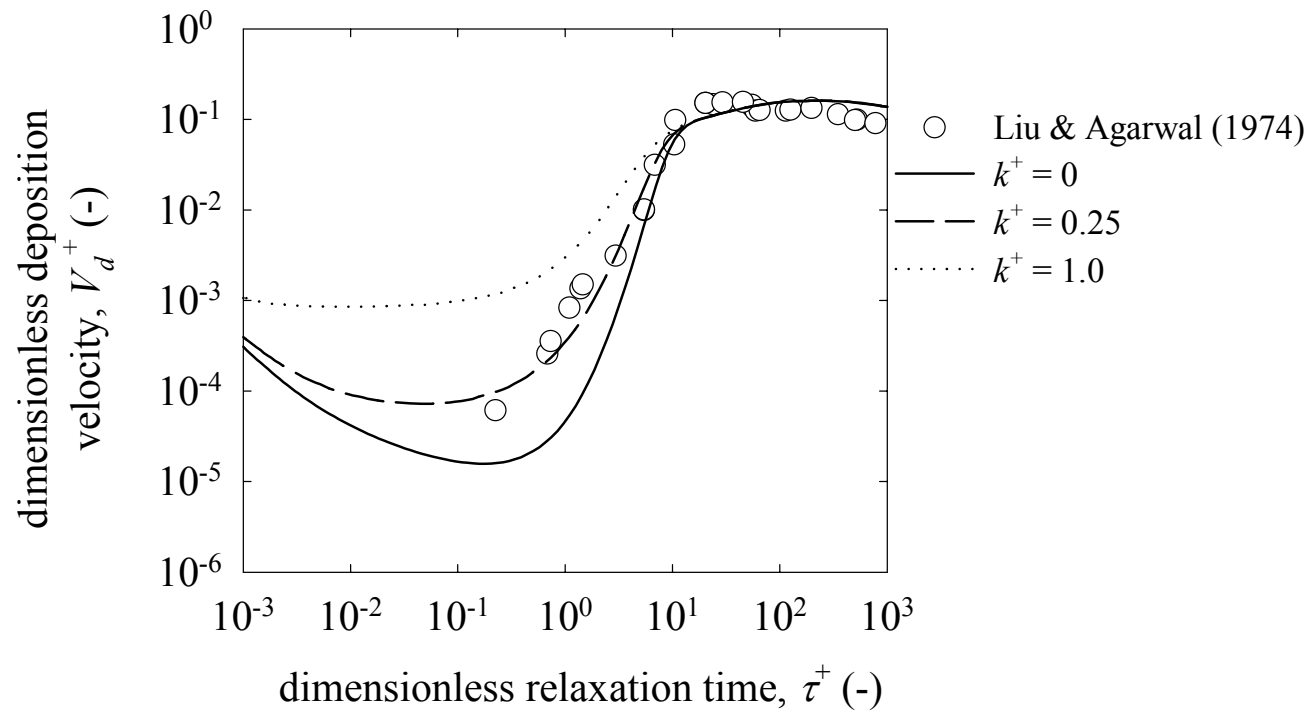

Figure 29. Application of the turbophoretic model of Guha (1997) with different roughness values to the conditions of the experiments of Liu \& Agarwal (1974).

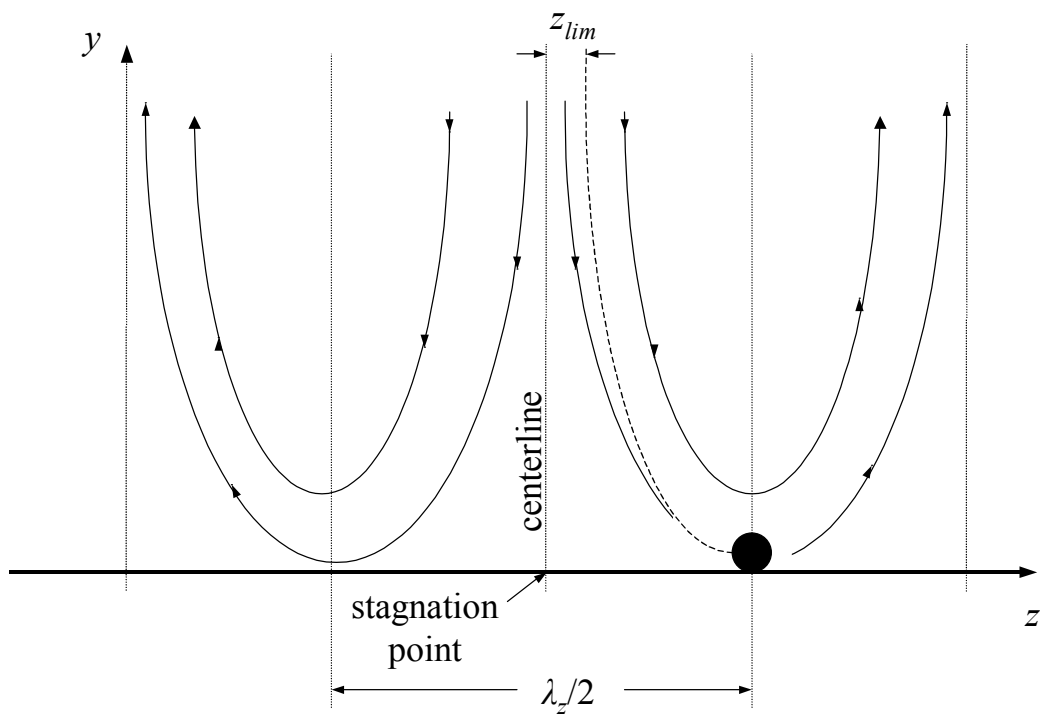

Figure 30. Schematic of near-wall turbulence modeling by two-dimensional stagnation point flow in sublayer models. A particle depositing at the limiting trajectory is also shown. 


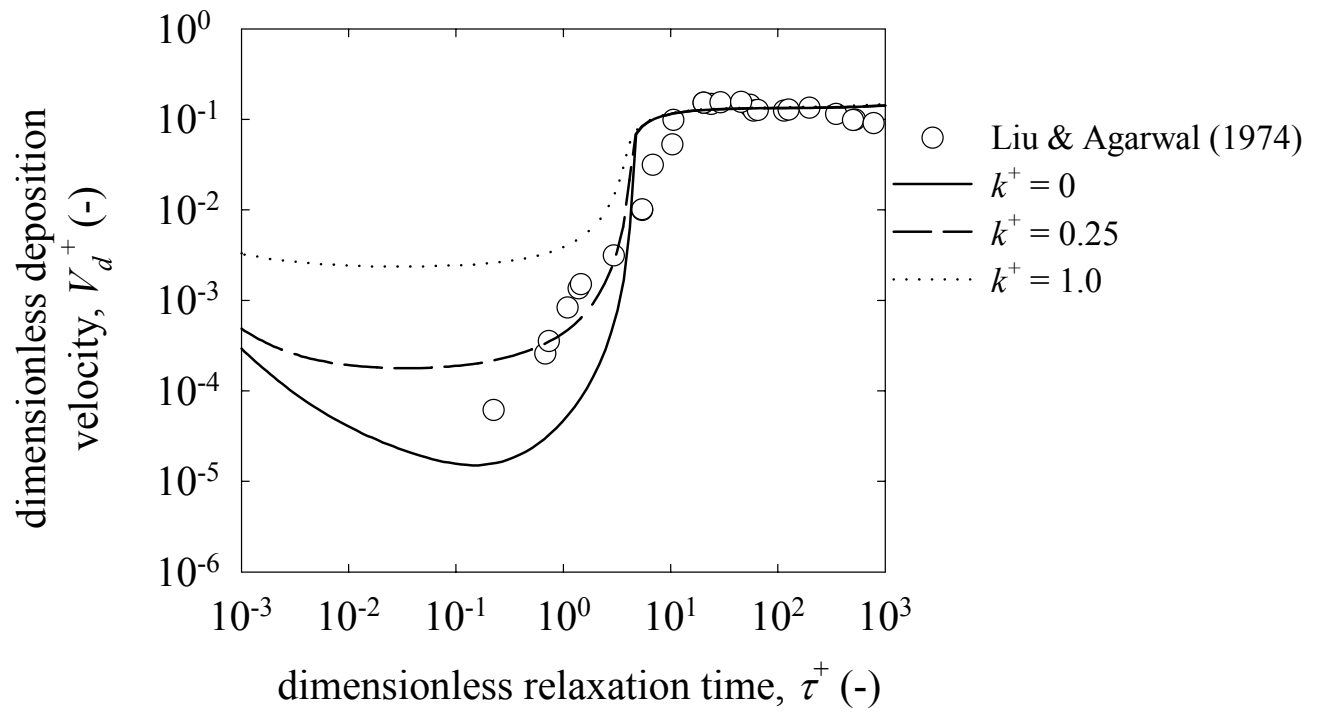

Figure 31. Application of the sublayer model of Fan \& Ahmadi (1993) with different roughness values to the conditions of the experiments of Liu \& Agarwal (1974).

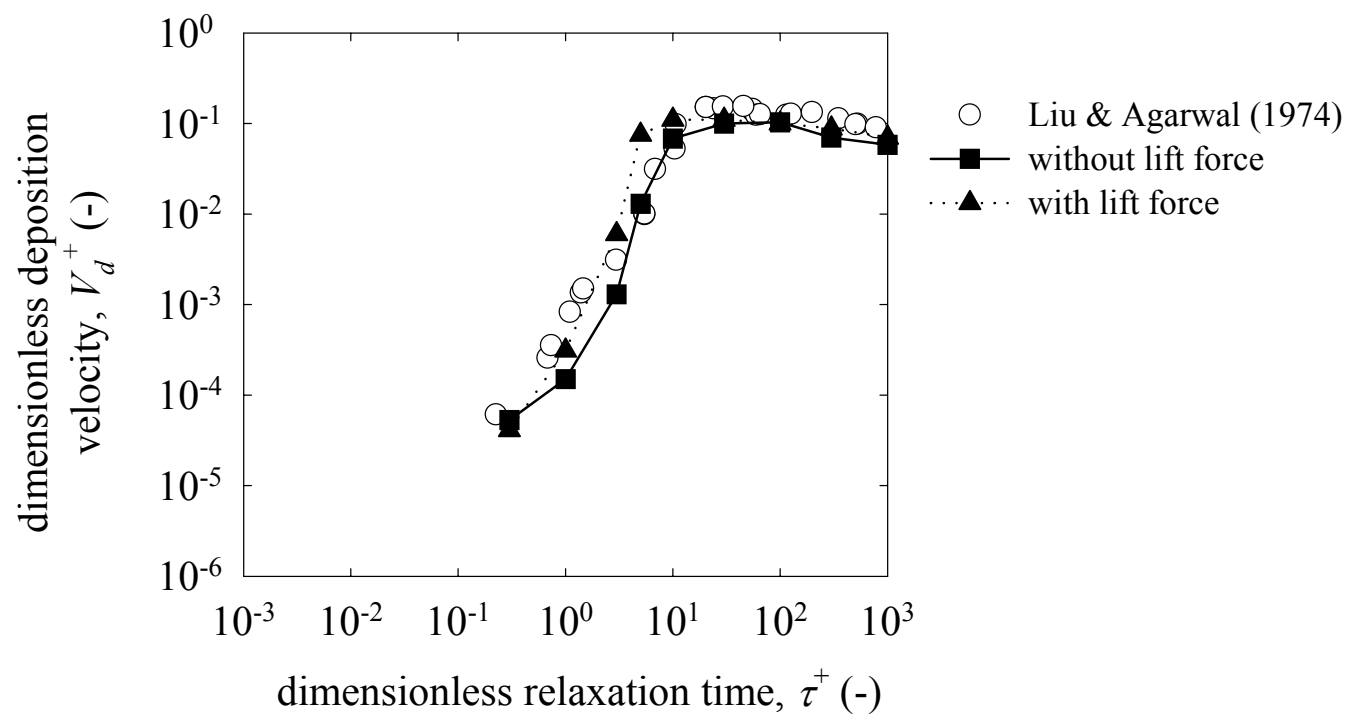

Figure 32. Results for deposition velocity to a smooth vertical surface by the Lagrangian simulation of Kallio \& Reeks (1989) with and without the lift force. 


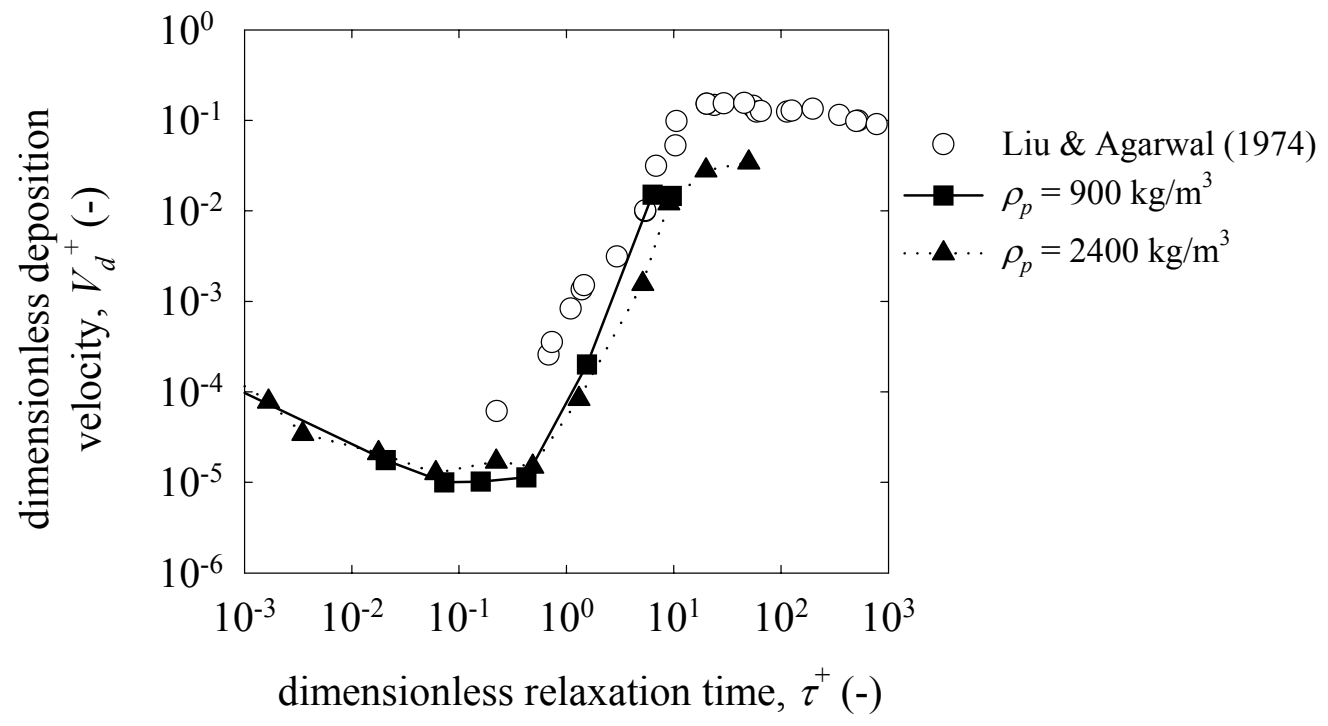

Figure 33. Results for deposition velocity to a smooth vertical surface by the Lagrangian simulation of Li \& Ahmadi (1993a) for particle densities of 900 and $2400 \mathrm{~kg} / \mathrm{m}^{3}$.

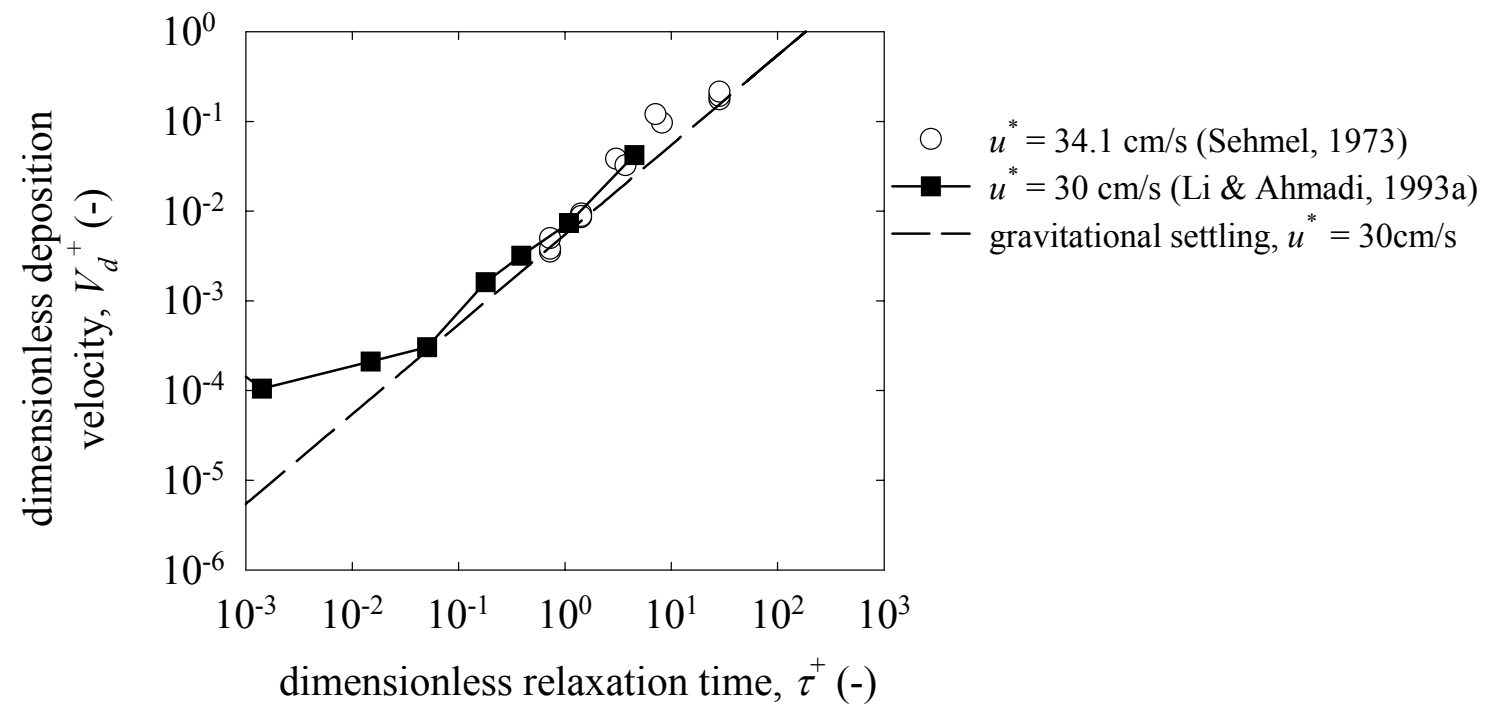

Figure 34. Results for deposition velocity to a smooth floor surface by the Lagrangian simulation of Li \& Ahmadi (1993a) compared to the similar experiments of Sehmel (1973). 


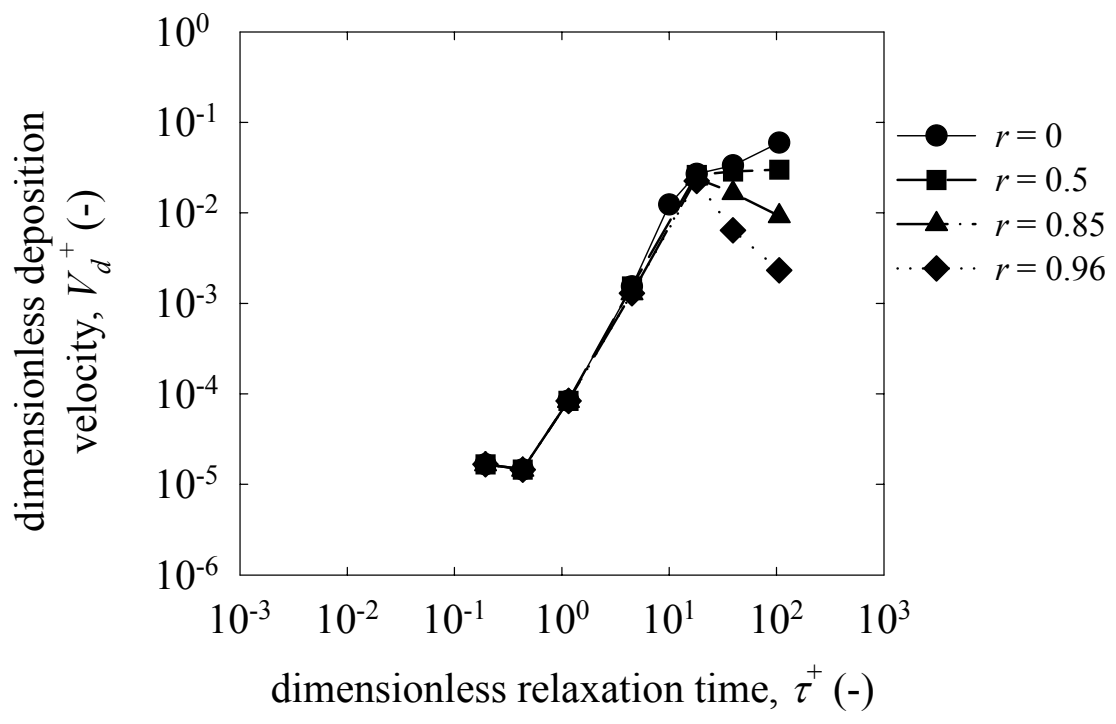

Figure 35. Results for deposition velocity to a smooth vertical surface by the Lagrangian simulation of Li \& Ahmadi (1993a) allowing for particle bounce with different restitution coefficients, $r$.

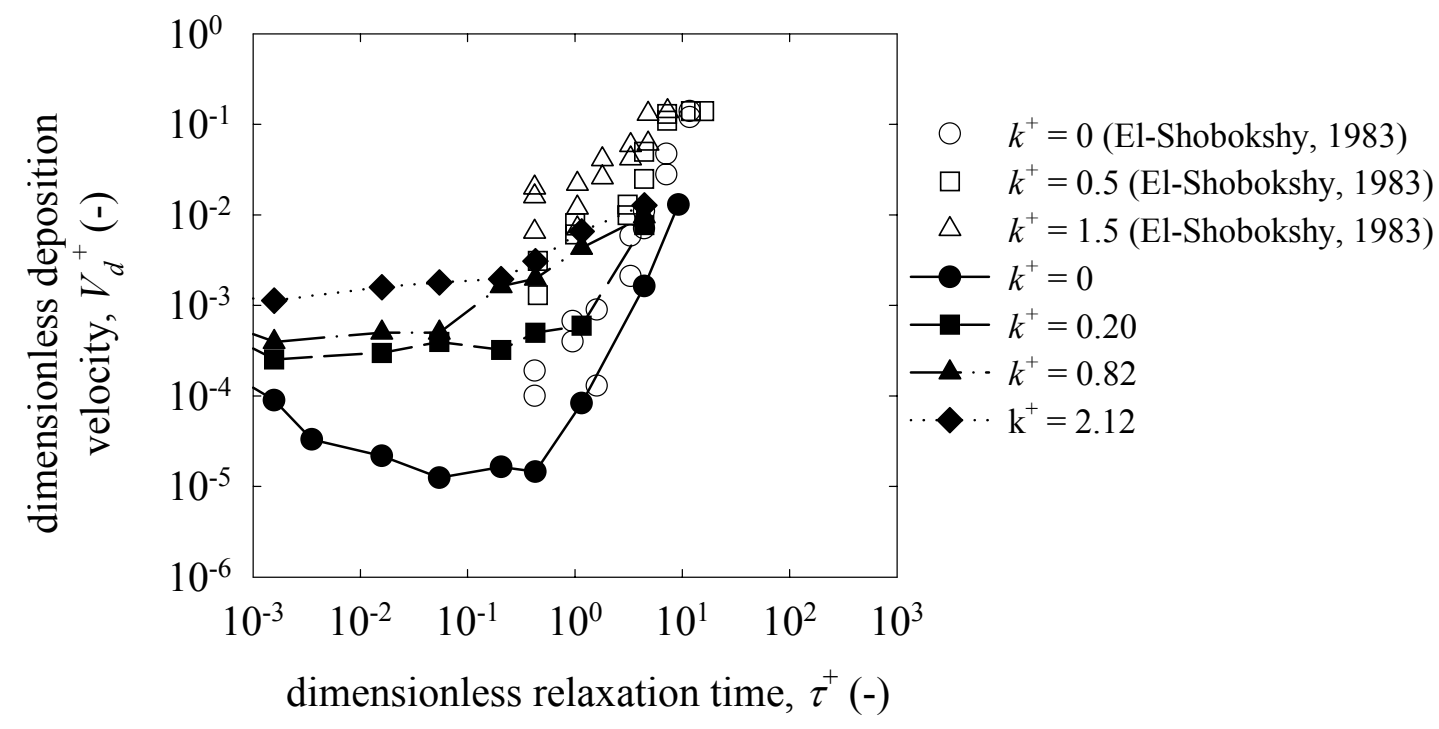

Figure 36. Results for deposition velocity to smooth and rough vertical surfaces by the Lagrangian simulation of Li \& Ahmadi (1993b) with four different roughness values. 


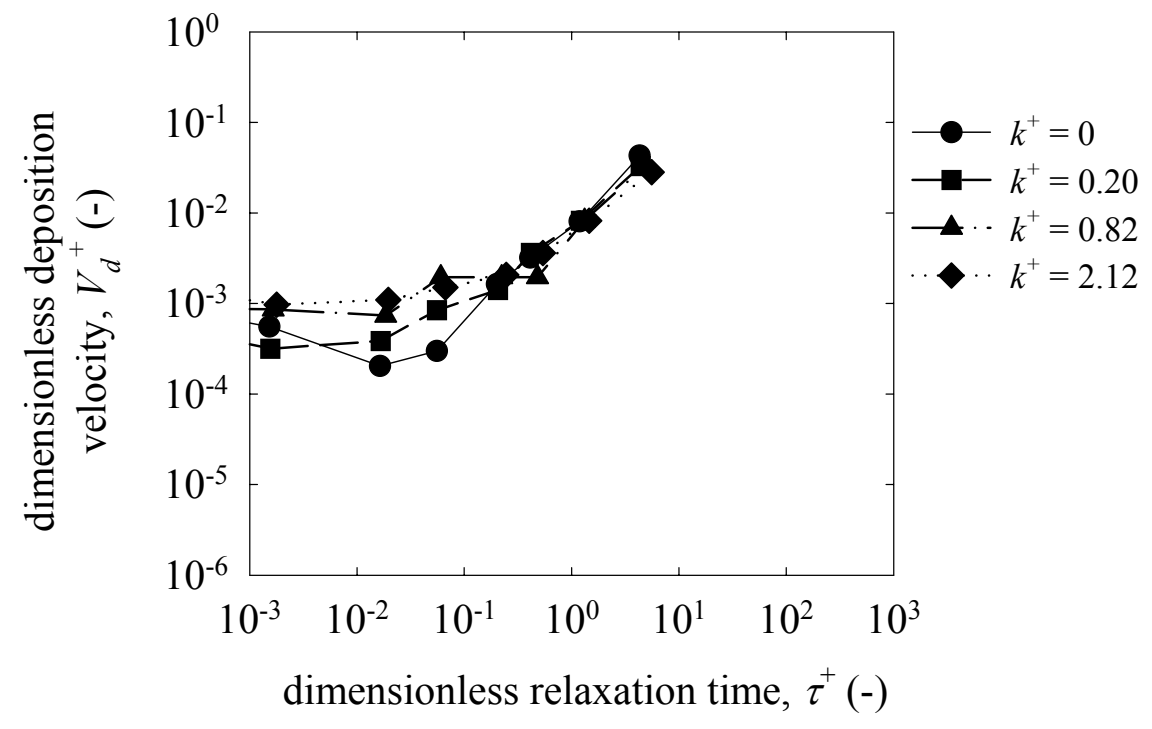

Figure 37. Results for deposition velocity to rough horizontal floor surfaces by the Lagrangian simulation of Li and Ahmadi (1993b) with four different roughness values.

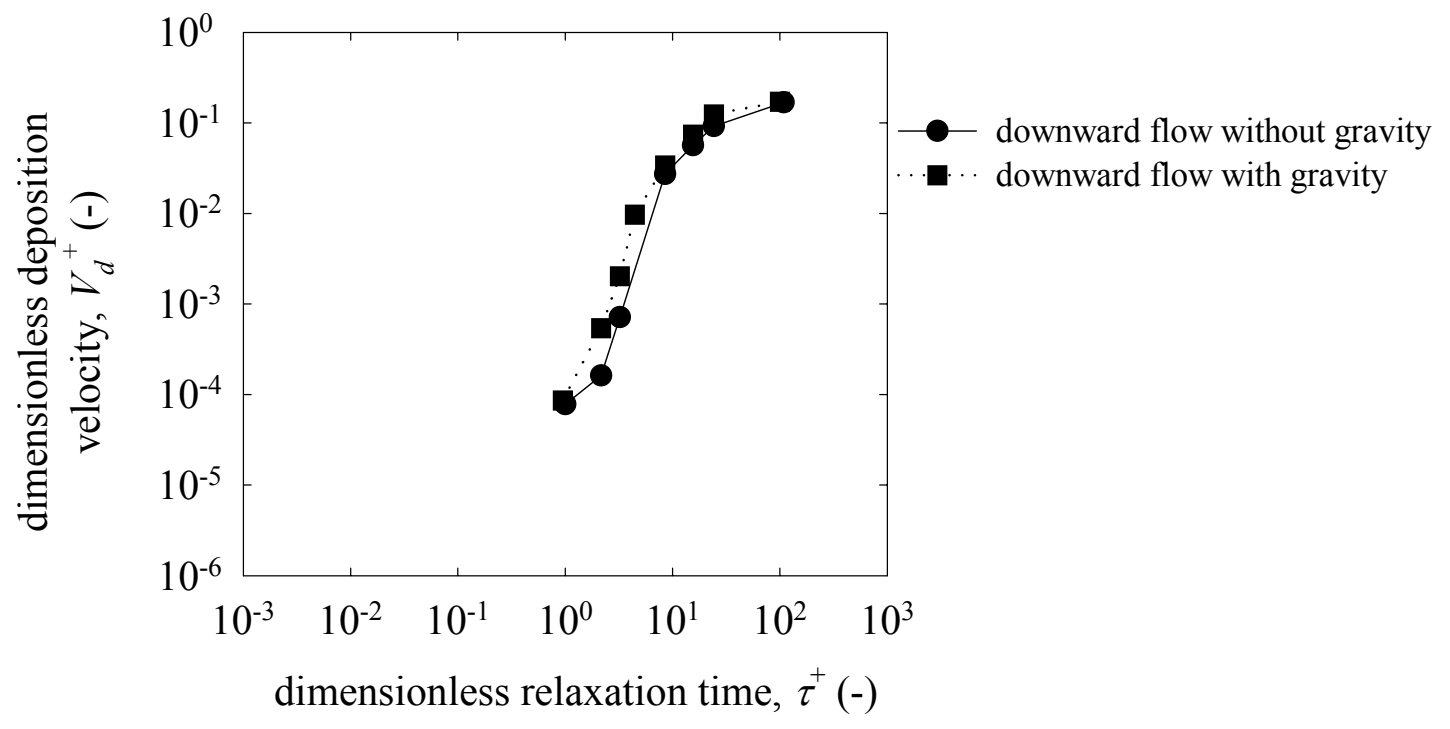

Figure 38. Results for deposition to a smooth vertical surface in the simulation of Chen \& Ahmadi (1997) showing the influence of gravity in a vertical flow through the shearinduced lift force. 


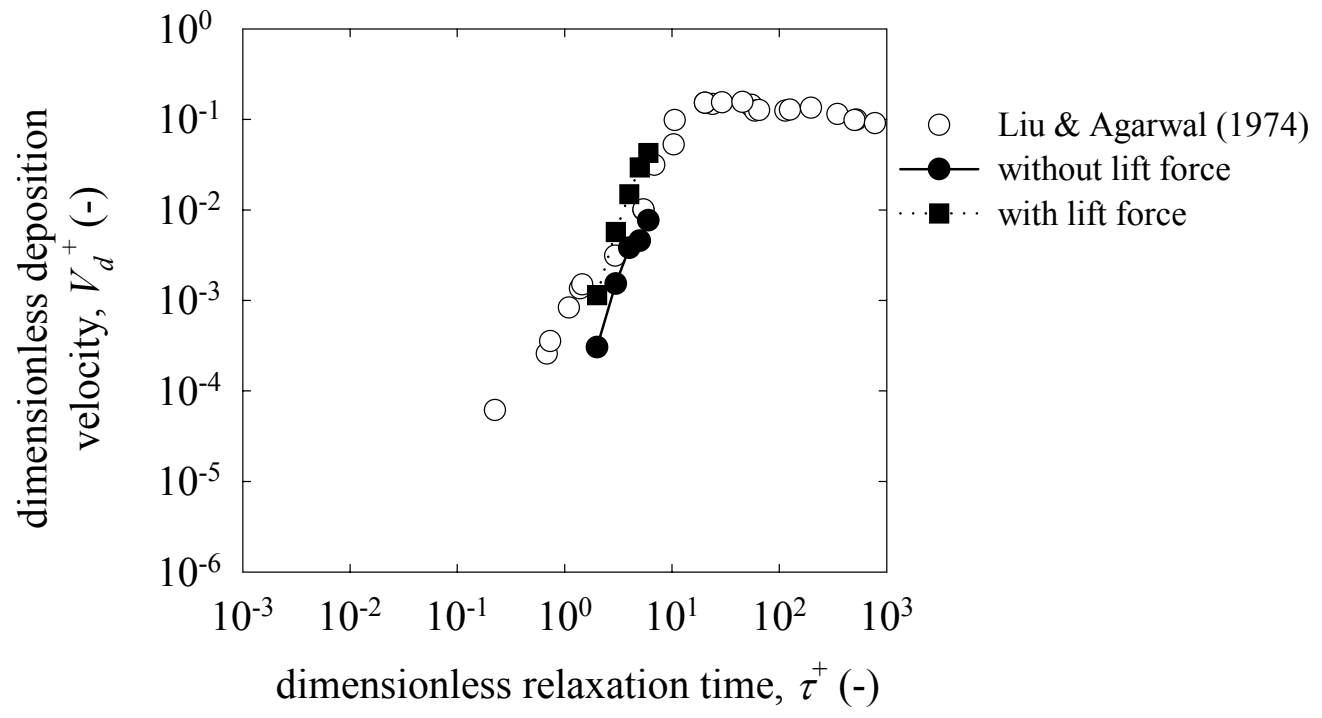

Figure 39. Results of the DNS-Lagrangian simulation of McLaughlin (1989) with and without the lift force compared to the experiments of Liu \& Agarwal (1974).

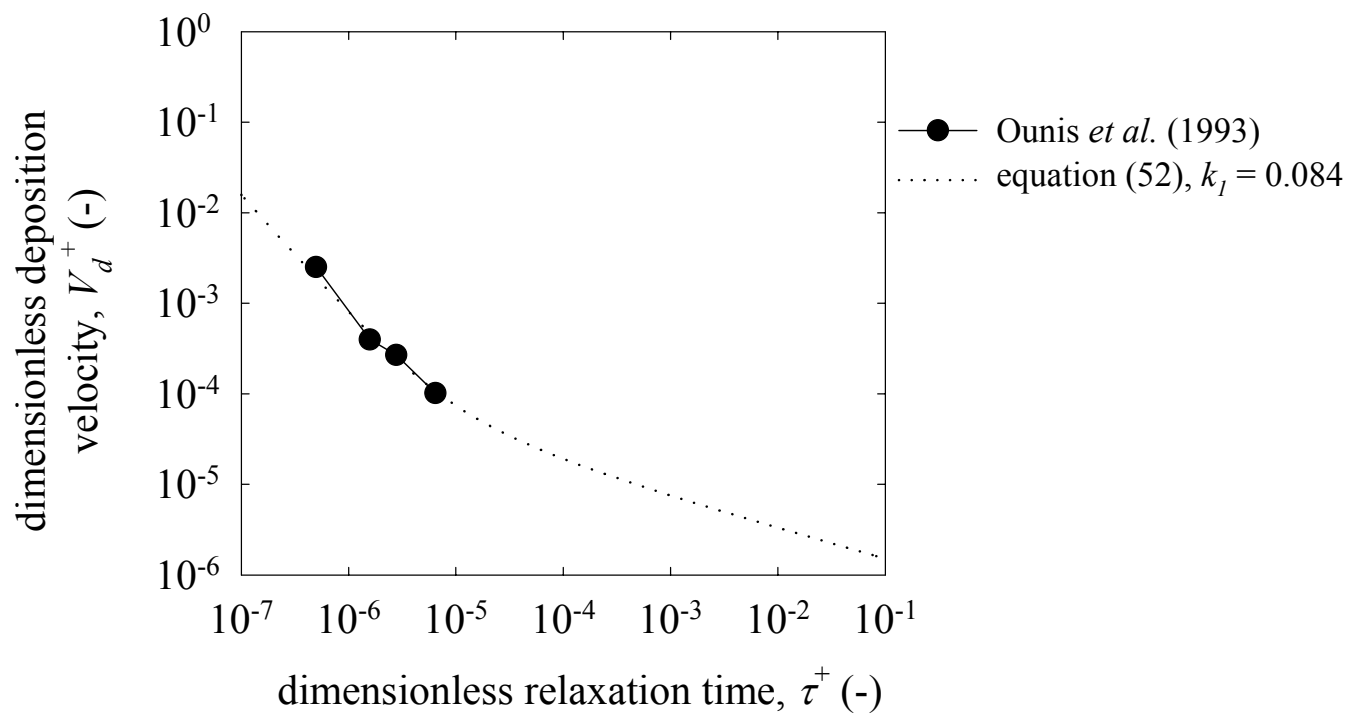

Figure 40. Results of the DNS-Lagrangian simulation of Ounis et al. (1993) for particles in the diffusive regime compared to the empirical equation (52) with $k_{1}=0.084$. 


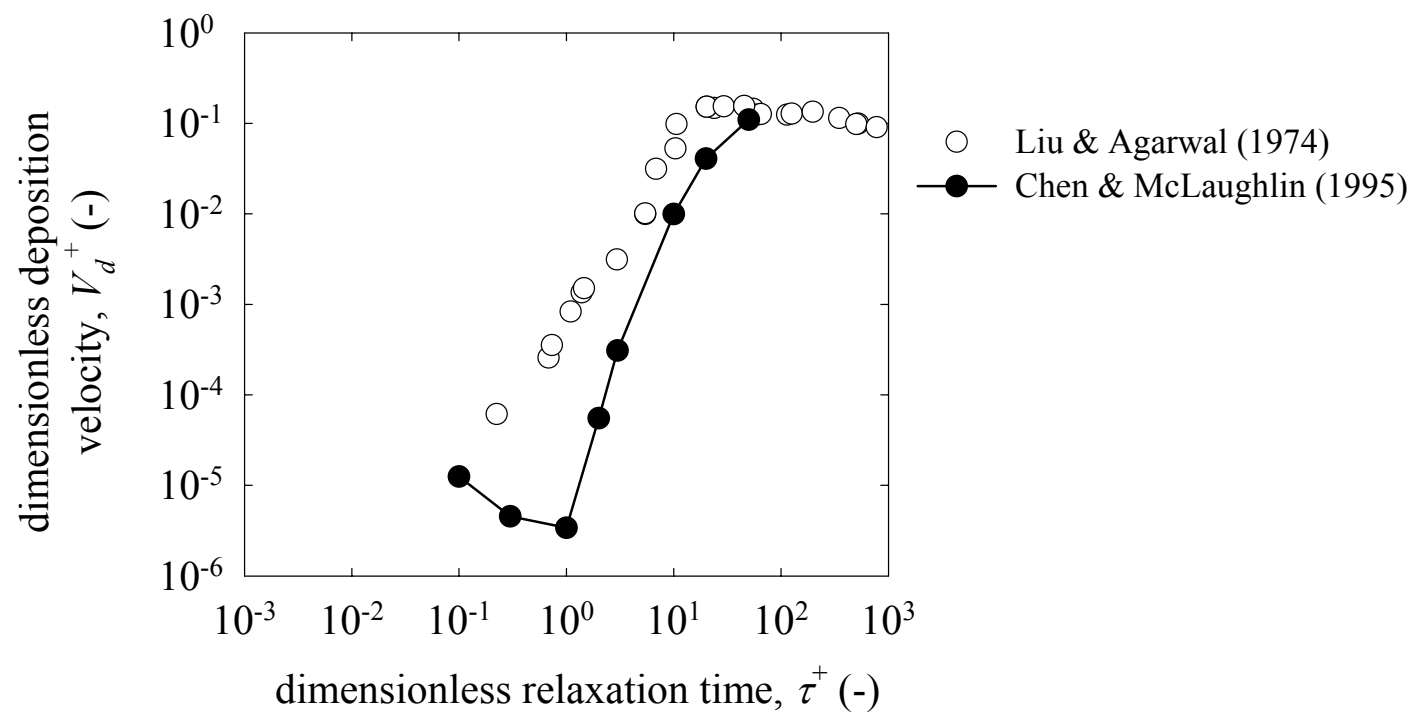

Figure 41. Results of the DNS-Lagrangian simulation of Chen \& McLaughlin (1995) with wall-corrected drag and optimum lift compared to the experiments of Liu \& Agarwal (1974).

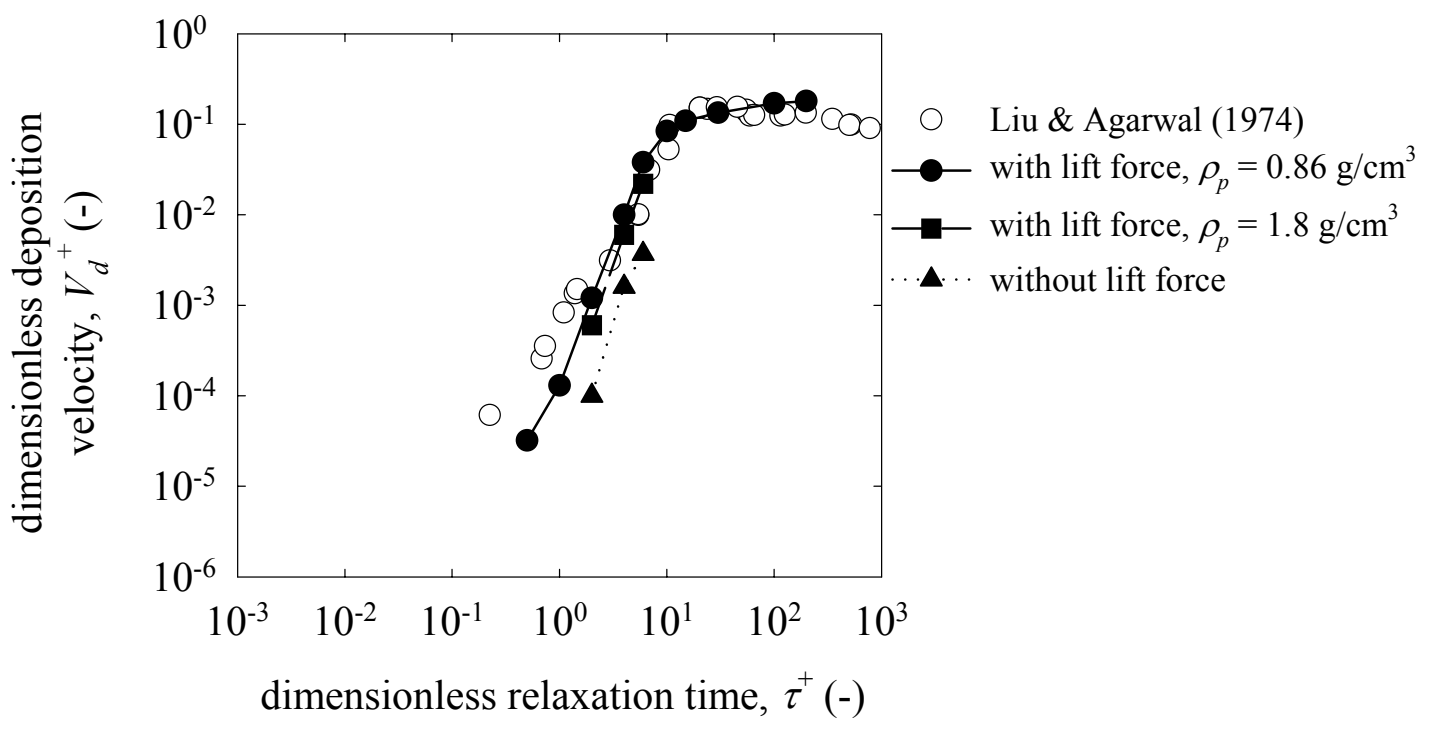

Figure 42. Results of the LES-Lagrangian simulation of Wang \& Squires (1996b) with and without the lift force compared to the experiments of Liu \& Agarwal (1974). 


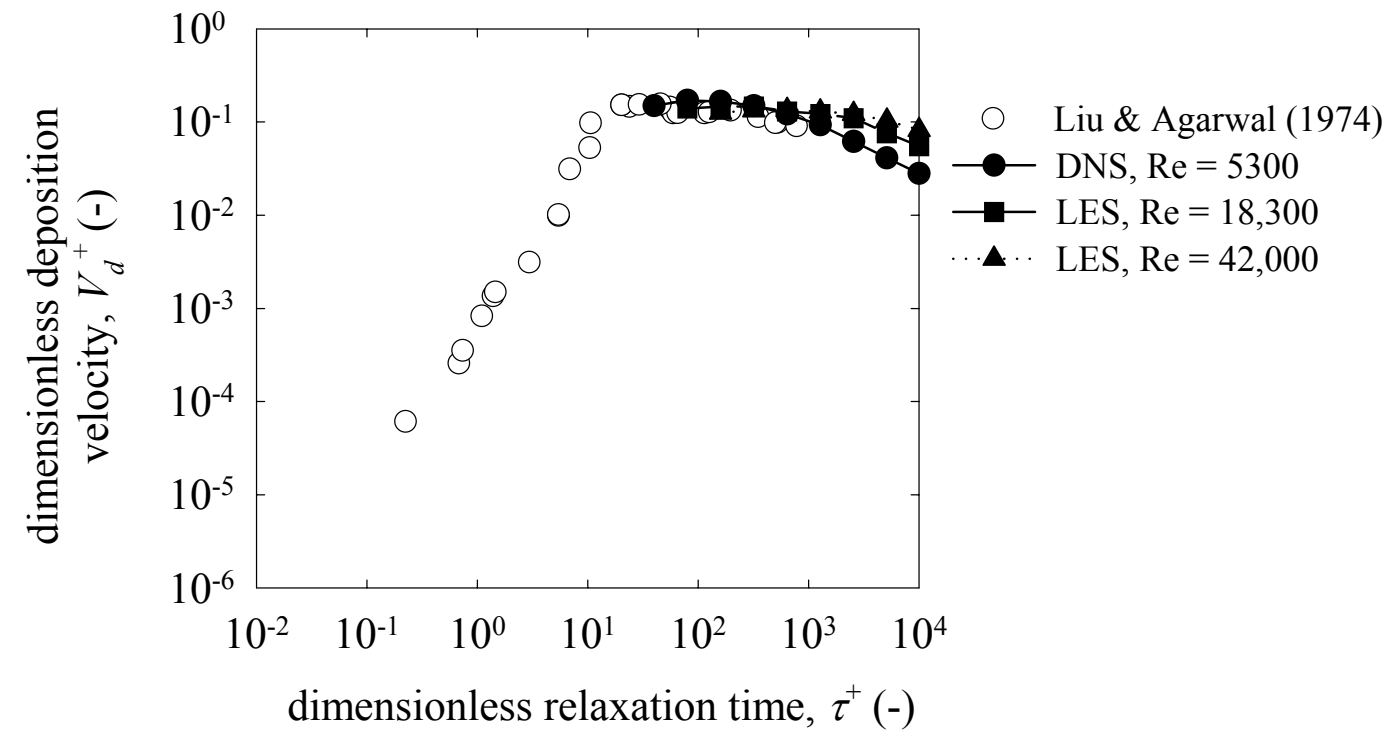

Figure 43. Results of the DNS- and LES-Lagrangian simulations of Uijttewaal \& Oliemans (1996) with drag force only compared to the experiments of Liu \& Agarwal (1974).

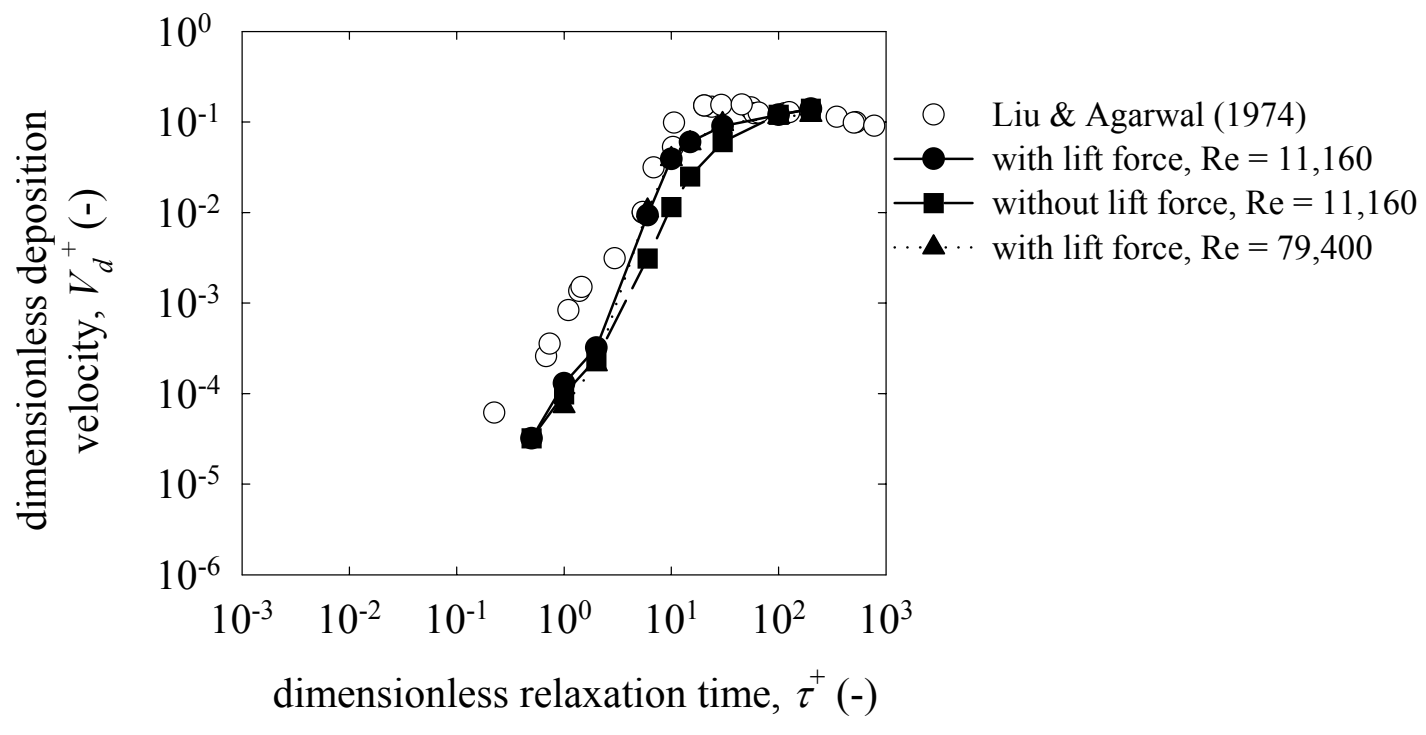

Figure 44. Results of the LES-Lagrangian simulation of Wang et al. (1997) with and without the optimum lift force compared to the experiments of Liu \& Agarwal (1974). 


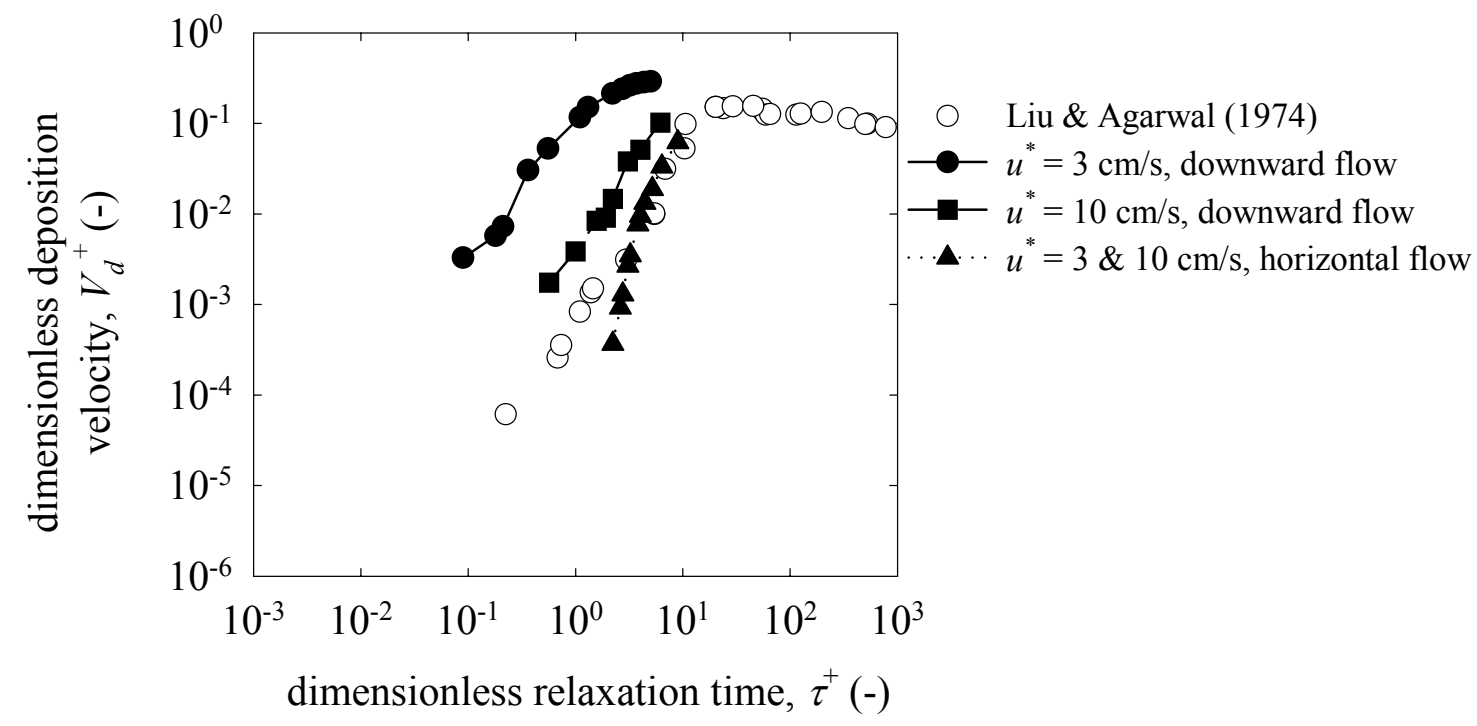

Figure 45. Results of the DNS-Lagrangian simulation of Zhang \& Ahmadi (2000) with downward and horizontal flow for deposition to a vertical wall surface at low friction velocities compared to the experiments of Liu \& Agarwal (1974).

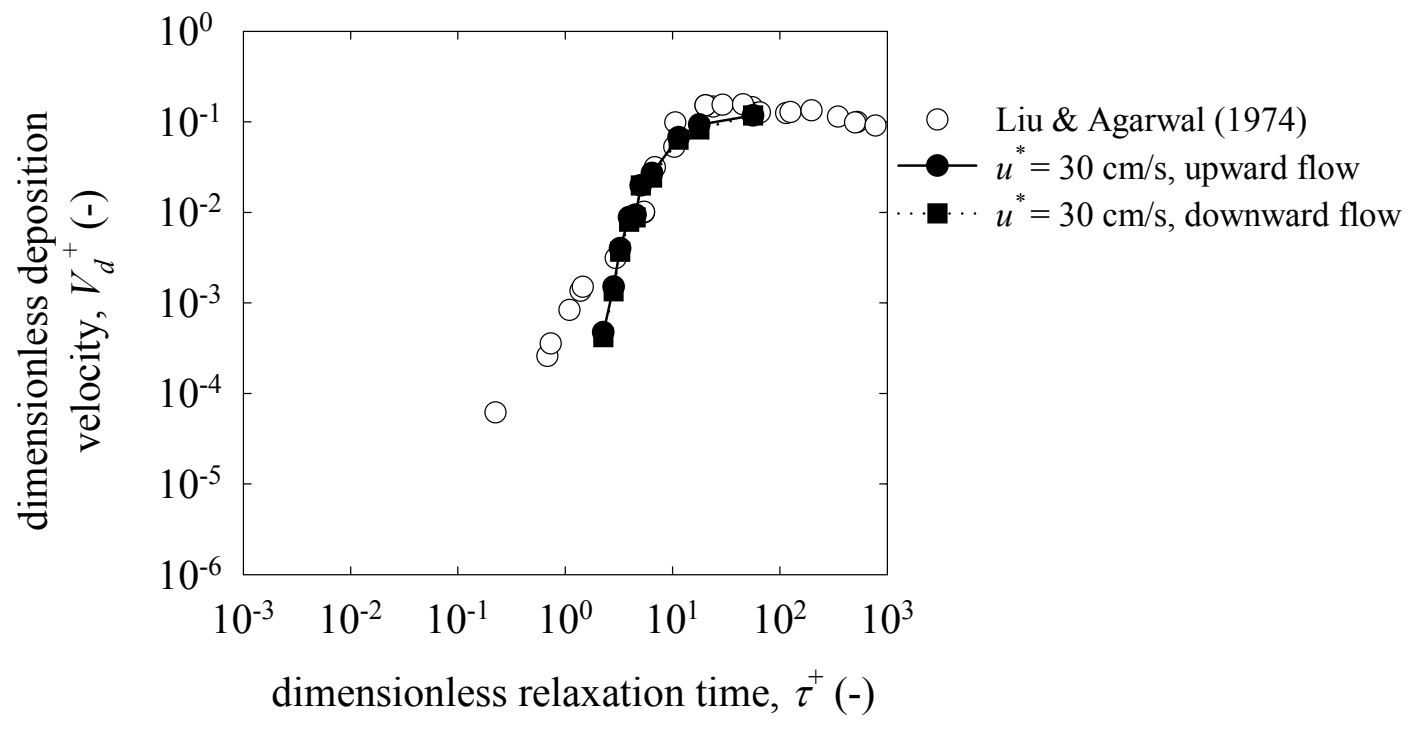

Figure 46. Results of the DNS-Lagrangian simulation of Zhang \& Ahmadi (2000) for deposition to a vertical wall from upward flow and downward flow at high friction velocities compared to the experiments of Liu \& Agarwal (1974). 


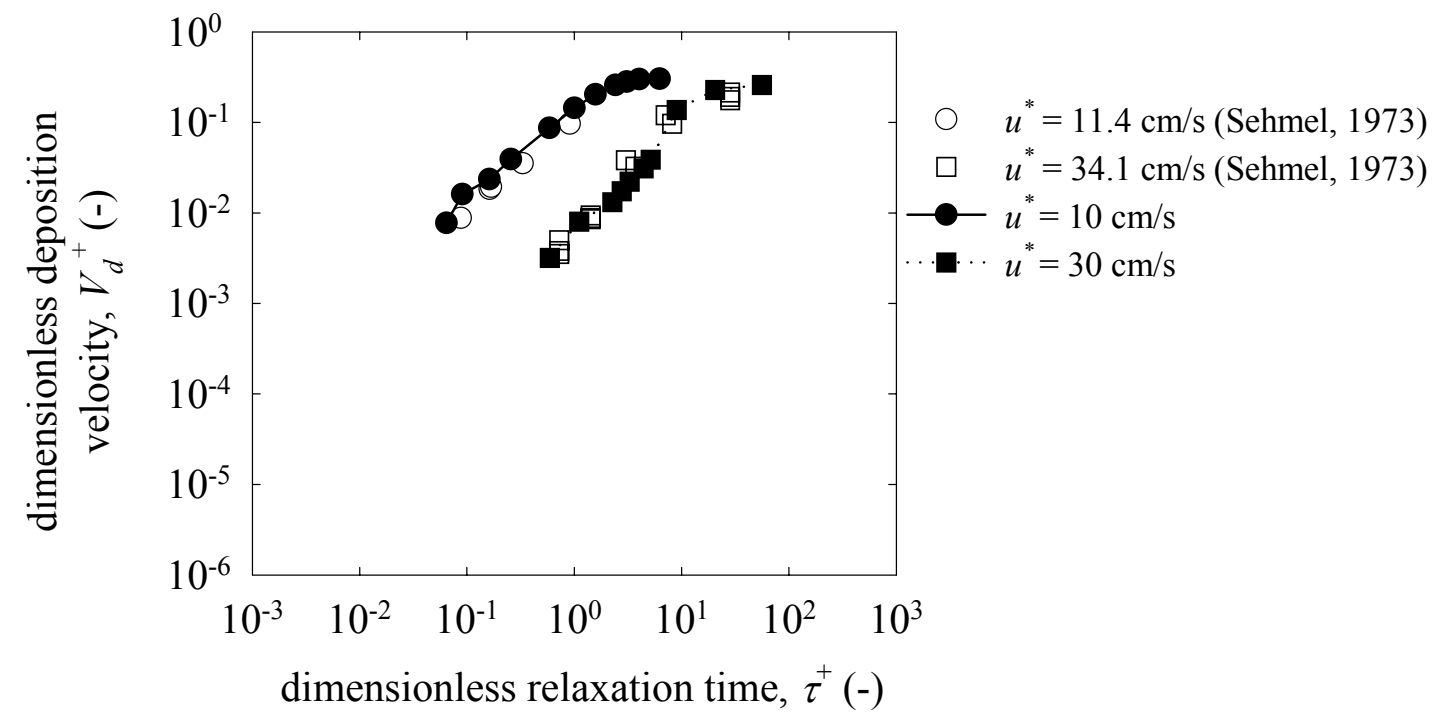

Figure 47. Results of the DNS-Lagrangian simulation of Zhang and Ahmadi (2000) for deposition to a horizontal floor at two friction velocities compared to the analogous experiments of Sehmel (1973).

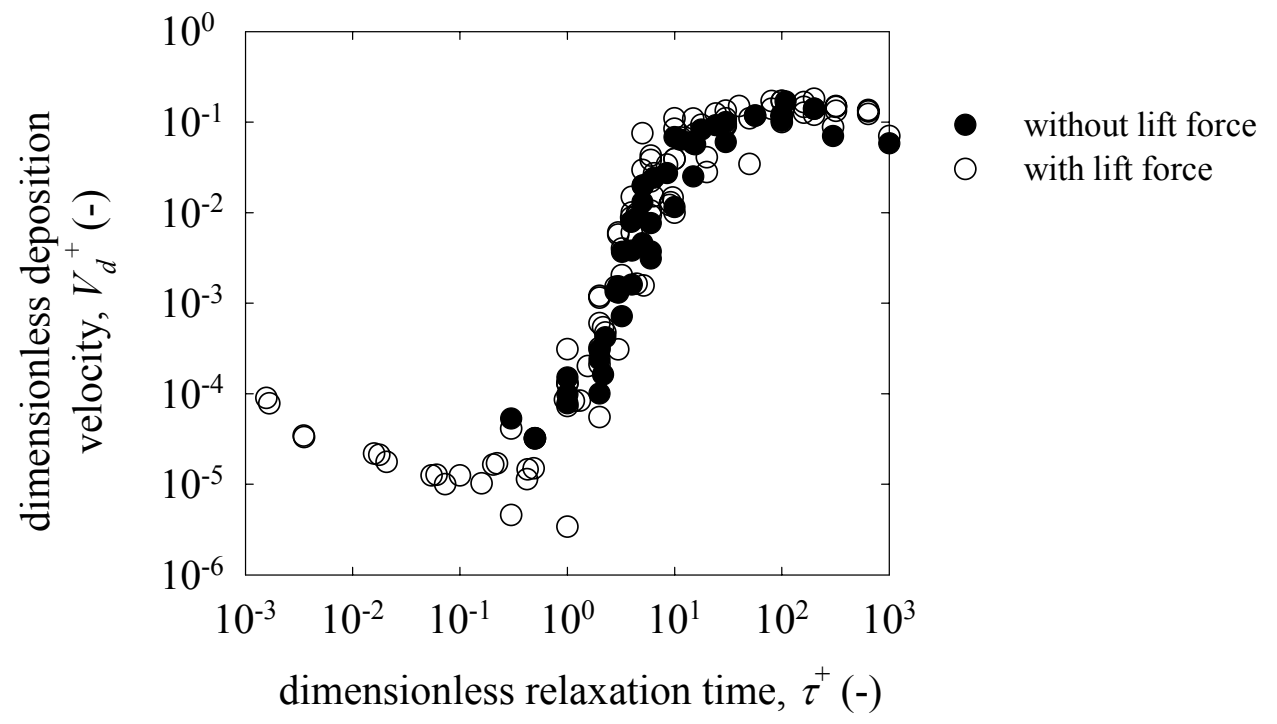

Figure 48. Summary of results for deposition to smooth vertical surfaces from several Lagrangian simulations. 


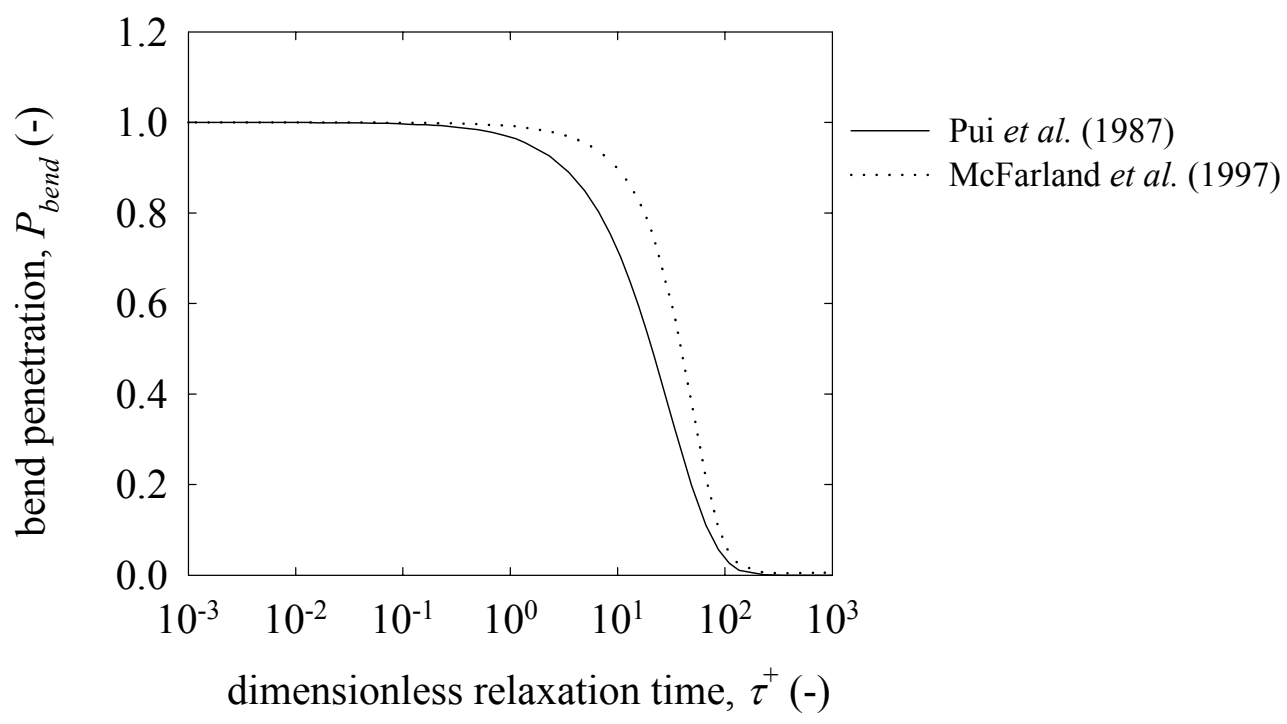

Figure 49. Comparison of empirical model predictions for particle penetration through a $90^{\circ}$ bend in a $15-\mathrm{cm}$ square duct at a velocity of $5 \mathrm{~m} / \mathrm{s}$. 